\title{
Characterization and construction of max-stable processes
}

\author{
Dissertation \\ zur Erlangung des \\ mathematisch-naturwissenschaftlichen Doktorgrades \\ Doctor rerum naturalium \\ der Georg-August-Universität Göttingen \\ im Promotionsprogramm Mathematik \\ der Georg-August-University School of Science (GAUSS)
}

vorgelegt von

Kirstin Strokorb

aus Stendal

Göttingen 2013 


\section{Betreuungsausschuss}

Jun.-Prof. Dr. Andrea Krajina $\left(\mathrm{IMS}^{1}\right)$

Prof. Dr. Robert Schaback $\left(\mathrm{NAM}^{2}\right)$

Mitglieder der Prüfungskommission

Referentin: Jun.-Prof. Dr. Andrea Krajina $\left(\mathrm{IMS}^{1}\right)$

Korreferent: $\quad$ Prof. Dr. Robert Schaback $\left(\mathrm{NAM}^{2}\right.$ )

Weitere Mitglieder: Prof. Dr. Dominic Schuhmacher $\left(\mathrm{IMS}^{1}\right)$

PD Dr. Ulf-Rainer Fiebig $\left(\right.$ IMS $\left.^{1}\right)$

Prof. Dr. Gert Lube (NAM²)

Prof. Dr. Victor Pidstrygach $\left(\mathrm{Ml}^{3}\right)$

${ }^{1}$ Institut für Mathematische Stochastik,

${ }^{2}$ Institut für Numerische und Angewandte Mathematik,

${ }^{3}$ Mathematisches Institut

(jeweils Fakultät für Mathematik und Informatik, Georg-August-Universität Göttingen)

Tag der mündlichen Prüfung: 2. Juli 2013 




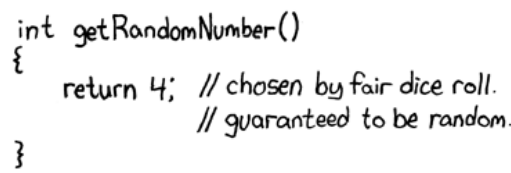

( http://xkcd.com/221/)

\section{Acknowledgements}

Now that this thesis is finally written, I wish to express my sincere gratitude to those who helped me in these past three years - scientifically and personally.

First of all, I am truly indepted to my supervisor Prof. Dr. Martin Schlather who was always available whenever I needed guidance through this project, even in busy times. I am grateful for numerous discussions in a friendly and creative atmosphere, for his stimulating enthusiasm, for his trust and patience allowing me to find and pursue my own interests and in particular, for his constant encouragement. Likewise, I very warmly thank Jun.-Prof. Dr. Andrea Krajina for unconditionally taking over my supervision and supporting and encouraging me in these last three months, and my co-supervisor Prof. Dr. Robert Schaback, who always has an open door to enter, for sharing his knowledge and experience.

I appreciate very much working together with Dr. Felix Ballani on various issues of chapter 4 of this thesis, and warmly thank him for his help and fruitful discussions during his visit in Göttingen and while being my host in Freiberg. I am indepted to PD Dr. Ulf-Rainer Fiebig for very valuable comments on the very final version of this thesis. Moreover, I wish to thank Dr. Vadim Alekseev, Martin Callies, Dr. Anja Janßen, Jun.-Prof. Dr. Zakhar Kabluchko, Prof. Dr. Ilya Molchanov, Yiannis Papastathopoulos Ph.D., Robert Schieweck, Dr. Marie Schmidt, Henrik Schumacher and Prof. Jonathan Tawn for spontaneously answering emails and giving valuable support, hints and comments. I am much obliged to Julian Rüger for the afternoons he spent on several computer problems.

I am thankful for the opportunity to research as a member of the Research Training Group 1023 "Identification in Mathematical Models: Synergy of Stochastic and Numerical Models" and gratefully acknowledge the benefits and generous financial support through the German Research Foundation DFG. I look back at pleasant memories of the annual Goslar workshops and excellent lecture series. I also enjoyed excellent lectures and seminars given by PD Dr. Doris Fiebig, Prof. Dr. Gert Lube and Prof. Dr. Anja Sturm. Further, I would like to thank my committee members PD Dr. Ulf-Rainer Fiebig, Prof. Dr. Gert Lube, Prof. Dr. Victor Pidstrygach and Prof. Dr. Dominic Schumacher. I guess it is due to my Diploma supervisor Prof. Dr. Victor Pidstrygach and my teacher Dr. Wolfgang Ludwicki that I am "always coming back to geometry", as a friend once said. 
I owe pleasant times and conversations to my colleagues, fellow students and friends. Among many others it is my pleasure to point out at least a few. Special thanks go to Karola Kombrink and Marco Oesting for their warm welcome in the institute, to Katharina Burmeister, Ulrike Ober, Greta Richter, Henrik Schumacher, Kristin Stroth and Carsten Thiel for cheering me up from time to time. I enjoyed interesting conversations with Dong-Du Mai, Juliane Manitz, Johannes Martini and Thomas Rippl. And indeed, I am truly more than grateful to Sebastian Engelke, Alexander Malinowski and Marco Oesting for being such an amicable working group. Thank you for inspiring discussions, for reading various parts of this manuscript beforehand and providing critical remarks and valuable comments.

Finally, I owe my deepest gratitude to my family for their unconditional support and love, especially to my sister Christiane and to my husband Ulrich. Thank you so much. 


\section{Contents}

\begin{tabular}{ll}
\hline Introduction & 1
\end{tabular}

$\begin{array}{lll}1 & \text { Preliminaries } & 7\end{array}$

1.1 Max-stable processes $\ldots \ldots \ldots \ldots \ldots$. . . . . . . . . . . . . 7

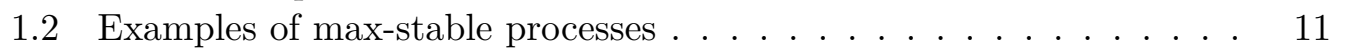

2 Max-stable processes parametrized by their extremal coefficients $\quad 15$

2.1 Extremal coefficient functions . . . . . . . . . . . . . 15

2.2 A consistent max-linear model $\ldots \ldots \ldots \ldots$. . . . . . . . . 17

2.3 Negative definiteness of ECFs . . . . . . . . . . . . . . . . . . . . . . . . 20

2.4 Consequences of negative definiteness. . . . . . . . . . . . . . . 24

2.4 .1 Convexity and compactness $\ldots \ldots \ldots \ldots . \ldots . \ldots 24$

2.4 .2 Spectral representations . . . . . . . . . . . . . . . 25

2.4 .3 Triangle inequalities and the operation of Bernstein functions 28

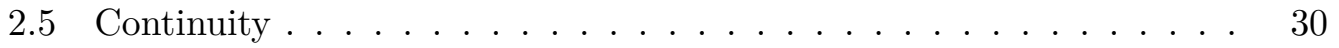

2.6 Dependency sets $\ldots \ldots \ldots \ldots$

2.7 Directional ECFs . . . . . . . . . . . . . . . . . . . . . . . . . . . 4

2.7 .1 Consequences of negative definiteness . . . . . . . . . . . 44

2.7 .2 Dependency sets . . . . . . . . . . . . . . . . . 45

$\begin{array}{lll}3 \text { A characterization of tail correlation functions } & 51\end{array}$

3.1 Tail correlation functions $\ldots \ldots \ldots \ldots \ldots$. . . . . . . . . 51

3.2 TCFs are realized by max-stable processes $\ldots \ldots \ldots \ldots$

3.2 .1 Proof of Theorem 3.2 .4 using ECFs $\ldots \ldots \ldots \ldots$

3.3 Operations on TCFs $\ldots \ldots \ldots \ldots \ldots$. . . . . . . . . . 57

3.4 Characterization by finite-dimensional inequalities . . . . . . . . . 58

3.4.1 The convex polytopes $\Theta(M)$ and $\mathrm{TCF}(M)$. . . . . . . . 60

3.5 Extremal TCFs . . . . . . . . . . . . . . . 66

3.6 TCFs compared to non-negative correlation functions . . . . . . . . 70

3.6 .1 Finite-dimensional inequalities . . . . . . . . . . . . 71

\begin{tabular}{|lll}
\hline & Max-stable processes sharing the same tail correlation function & $\mathbf{7 5}$
\end{tabular}

$4.1 \quad$ Stationary max-stable processes and their TCFs . . . . . . . . . . 75 
4.2 Brown-Resnick vs. EG and EBG processes . . . . . . . . . . . . . . 77

4.3 Brown-Resnick vs. Mixed Moving Maxima processes . . . . . . . . . 79

4.3 .1 Identifying the classes $\ldots \ldots \ldots \ldots$. . . . . . . . . . . . . . . . . . . . . . .

4.3 .2 Comparing the classes $\ldots \ldots \ldots \ldots \ldots$

$4.3 .3 \quad$ Sharp bounds for parametric subclasses. . . . . . . . . . . 85

4.4 Operations and Counterexamples . . . . . . . . . . . . . 86

4.4 .1 Turning bands . . . . . . . . . . . . . . . . . 86

4.4 .2 Multiplication with the Gneiting class $H_{d} \ldots \ldots$. . . . . . 89

4.4 .3 Counterexamples . . . . . . . . . . . . . . . . . 90

4.5 Proofs and plots $\ldots \ldots \ldots \ldots$

4.5 .1 Derivation of expressions in tables. . . . . . . . . . . . . . 99

4.5 .2 Plots $\ldots \ldots \ldots \ldots \ldots$

\begin{tabular}{|lll}
5 & Representations of Brown-Resnick processes for group actions & 105
\end{tabular}

5.1 Brown-Resnick processes for group actions . . . . . . . . . . . 107

5.2 Mixed Moving Maxima for group actions. . . . . . . . . . . . . . . . 108

5.3 M3 representation for actions of compact groups . . . . . . . . . . . 109

5.4 Extremal log-Gaussian representation on the sphere . . . . . . . . . 115

\begin{tabular}{ll}
\hline Concluding remarks & 117
\end{tabular}

\begin{tabular}{ll}
\hline A Appendix & 119
\end{tabular}

A.1 Harmonic analysis on abelian semigroups . . . . . . . . . . . . . . . 119

A.2 Monotonicity properties of continuous functions . . . . . . . . . . . . 123

A.3 $\quad$ Brown-Resnick processes . . . . . . . . . . . . . . . . 125

A.4 Extremal points and the Krein-Milman-Theorem . . . . . . . . . . . 126

A.5 $\quad$ Auxiliary results $\ldots \ldots \ldots \ldots \ldots \ldots$

\begin{tabular}{llll}
\hline A.6 & Further members of the Gneiting class $H_{\infty}$ & $\ldots$
\end{tabular}$\ldots \ldots \ldots$

\begin{tabular}{ll}
\hline References & 133
\end{tabular}

\begin{tabular}{|l|l|}
\hline List of abbreviations and symbols & 139
\end{tabular}

\begin{tabular}{lr}
\hline Index & 145
\end{tabular} 
Not that the story need be long,

but it will take a long while to make it short.

(Henry David Thoreau)

\section{Introduction}

Max-stable processes arise as limits of normalized maxima of independent copies of stochastic processes and they provide a natural framework to model spatial extremal scenarios. Over the last decade, they have gained an increasing attention in applications such as hydrology and meteorology - see [7, 8, 69] for some recent examples. While the theory and inference of max-stable distributions is well-developed in the univariate case [4, 16, 23, 79], appropriate modelling of the multivariate and spatial dependence structure is still a difficult question. Although convenient structural results and dependency descriptors are available in terms of exponent measure, spectral measure, stable tail dependence function, dependency set or Pickands' dependence function in the multivariate case [4, 15, 66, 75, 79] and spectral representations involving Poisson point processes or extremal stochastic integrals in the spatial case [15, 35, 96], all of these quantities are rather complex. This makes them hard to be estimated from data, see [19, 21, 22] and the references therein for some approaches in a multivariate setting, and contrasts in particular Gaussian processes, where the dependence structure of a finite sample is finite-dimensionally parametrized by its covariance. Therefore, it is often necessary to consider simpler extremal dependence measures as well as parametrized subclasses of max-stable laws.

Dating back to [32, 89, 95, simpler summary statistics include the extremal coefficients [85, 90] and the (upper) tail dependence coefficients [4, 10, 14, 28, 86], the latter being a special case of the extremogram [14] and often considered as an analogue to the correlation function for extreme values. In this thesis, we capture the full set of extremal coefficients of a max-stable process $X=\left\{X_{t}\right\}_{t \in T}$ on some space $T$ in the so-called extremal coefficient function (ECF) $\theta$, and the full set of upper tail dependence coefficients in what we will call here tail correlation function $(T C F) \chi$. Other names for the TCF $\chi$ include $\chi$-measure [4, 10] or extremal coefficient function [29] (not to be confused with the ECF above). 
Roughly speaking, the ECF $\theta$ assigns to each finite subset $A$ of $T$ the effective number of independent variables among the collection $\left\{X_{t}\right\}_{t \in A}$ (with respect to extremal dependence), whereas the TCF $\chi$ assigns to each pair $(s, t) \in T \times T$ the probability of observing a large value at the location $s$ conditioned on the event that a large value has been observed at the location $t$. Both quantities are intimately connected and we will benefit from this connection particularly in Chapter 3 .

In [85] the set of multivariate ECFs is shown to be in a 1-1 correspondence with a set of multivariate max-linear distributions and thereby characterized in terms of a set of inequalities. An alternative proof for these inequalities is given in [66] and it is noticed therein that they in fact express a property called complete alternation. In this thesis, these ideas are generalized to a spatial setting in Chapter 2, which contains a complete characterization of the set of ECFs and the construction of a corresponding max-stable process. Thus, analogies to Gaussian processes and positive definite functions are revealed as follows: Among (zero mean) square integrable processes, the subclass of Gaussian processes takes a unique role, since it is in a 1-1 correspondence with the set of covariance functions, which are precisely the positive definite functions.

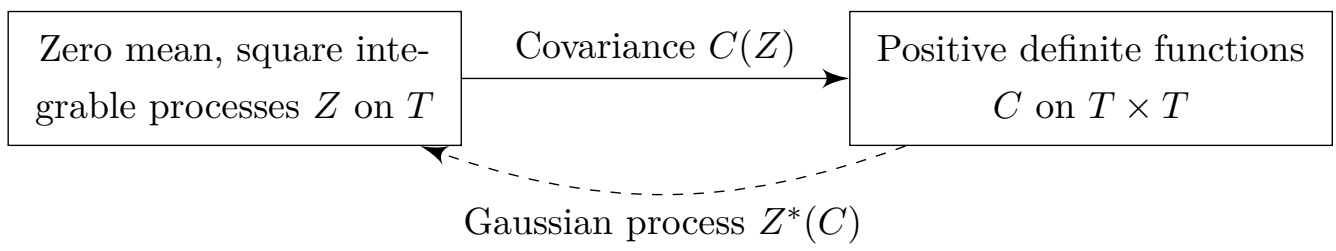

In case $T$ is a metric space, the Gaussian process $Z^{*}(C)$ is continuous in the mean square sense (and then also stochastically continuous) if and only if $C$ is continuous (on the diagonal) (cf. [81]). Well-known operations on the set of positive definite functions $C$, and hence on the corresponding Gaussian processes $Z^{*}(C)$, include convex combinations and pointwise limits. Moreover, Bernstein functions play an important role for the construction of Gaussian processes.

In our case, the role of (zero mean) square-integrable processes is taken by the simple max-stable processes and the crucial role of Gaussian processes is taken by a subclass of max-stable processes $X^{*}$, which is in fact the spatial generalization of the multivariate max-linear model of [85]. Alongside, generalizing the multivariate result [66, Corollary 1] to the spatial setting, we prove a characterization of the set of ECFs that can be illustrated in analogy to the above sketch. 


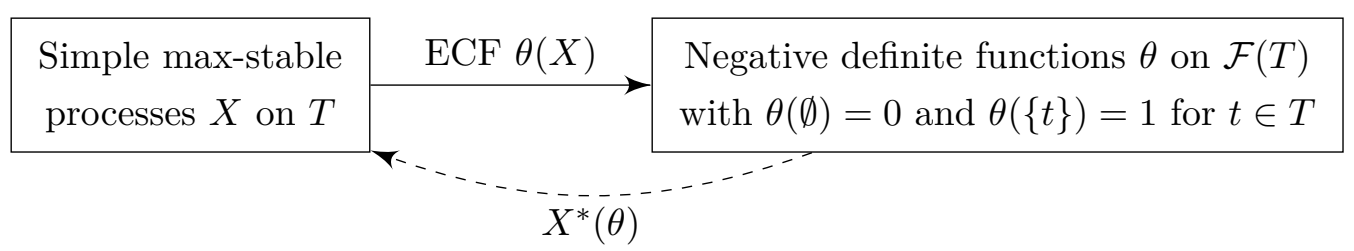

Here, $\mathcal{F}(T)$ denotes the set of finite subsets of $T$. Further, we derive a spectral representation for the corresponding process $X^{*}(\theta)$ and operations on ECFs that allow to build new ECFs from given ones, including convex combinations and pointwise limits. Certain "triangle inequalities" for $\theta$ from [11, Proposition 4] involving Bernstein functions are recovered and generalized. For $T$ being a metric space we discuss continuity of $\theta$ and its corresponding process $X^{*}(\theta)$. The dependency set $\mathcal{K}^{*}(\theta)$ of the process $X^{*}(\theta)$ is identified as intersection of halfspaces that are directly given by the ECF $\theta$, which leads to sharp inequalities for the finite dimensional distributions of arbitrary max-stable processes in terms of its ECF $\theta$. Finally, most of these results can be directly transferred to the more general directional ECF, which is not bound to diagonal data like the ECF.

Chapters 3 and 4 are concerned with the class of TCFs. A first structural result on TCFs was given by [20] showing that compactly supported TCFs of stationary dissipative max-stable processes on $\mathbb{Z}$ are in fact set correlation functions. Here, Chapter 3 exhibits the set of TCFs as an infinite-dimensional compact convex polytope and compares it to the set of non-negative correlation functions. It is well-known that TCFs are non-negative correlation functions, but not all non-negative correlation functions are TCFs. Nonetheless, both classes have desirable properties in common: (i) TCFs can be completely characterized by finite-dimensional inequalities and (ii) convex combinations, products and pointwise limits are admissable operations on TCFs (Chapter 3). Moreover, a well-known operator on correlation functions, the turning bands operator, is also applicable to TCFs (Chapter 4).

Up to the fourvariate case we compute the vertices and bounding hyperplanes of the polytope of TCFs explicitly. At least theoretically, there is an algorithm that provides vertices and inequalities of any order. As a byproduct, we may also formulate an algorithm that solves the inverse problem of finding a multivariate maxstable distribution realizing a prescribed TCF, even though we expect computations to be tedious and unfeasible already for low-dimensional cases. First attempts to tackle this inverse problem can be found in [28, 30].

TCFs can be defined also for processes that are not max-stable. We show that the set of all TCFs (of not necessarily max-stable processes) coincides with the class 
of TCFs stemming from max-stable processes. This is of particular importance for applications, as it ensures that the tail correlation of data, which are commonly assumed to be in the max-domain of attraction of some max-stable law, can be modelled appropriately by the tail correlation of some max-stable process.

Widely used flexible subclasses of stationary max-stable processes on $\mathbb{R}^{d}$ include (Mixed) Moving Maxima processes (cf. [70, 90, 91, 92]) parametrized by a (random) shape function, extremal Gaussian processes [83] parametrized by a correlation function and Brown-Resnick processes [52] parametrized by a variogram, the last two classes offering a link to Gaussian processes. In particular, the class of BrownResnick processes seems to be very promising for spatial applications due to its wide flexiblility while being conveniently parametrized by a bivariate quantity (cf. [18, 26]).

In Chapter 4 the TCFs of these processes are compared to each other. More precisely, the TCFs of Brown-Resnick processes with bounded variogram are compared to the TCFs of extremal Gaussian processes, whereas the TCFs of Brown-Resnick processes with unbounded variogram are compared to the TCFs stemming from Mixed Moving Maxima constructions. This case distinction is necessary due to the different ergodic properties of these processes, which is reflected in the tail behaviour of the respective TCFs. In the second case we focus on stationary isotropic processes and consider TCFs that decrease monotonously to zero. In fact, we observe several systematic coincidences of classes of TCFs, which shows that the TCF cannot distinguish between the respective processes. With regard to results from Chapter 3 we also find sharp bounds for the parameters of some well-known parametric families of correlation functions to be a TCF, including the Whittle-Matérn model. Additionally, we provide counterexamples to some naturally arising questions in this context.

In [52] it is shown that a rich class of Brown-Resnick processes can be represented as a Mixed Moving Maxima process, which is particularly relevant for simulation techniques [70, 71]. In this thesis, Chapter 5 deals with Brown-Resnick processes that are considered as group invariant processes on some space $T$ (instead of stationary processes on $\mathbb{R}^{d}$ ). Two representations of these processes are addressed a Mixed Moving Maxima representation that complements a result in [52] and an extremal log-Gaussian representation on the sphere. The Mixed Moving Maxima representation is derived when a suitable group action of some compact group is considered. The difficulty here does not lie in transferring the proof of [52] to the 
new situation, but in establishing a measurability result that might be of independent interest. In fact, this measurability problem does not occur in the previous case, since the question of a unique measurable argmax can be avoided by taking the (lexicographic) infimum which commutes with the translation action of $\mathbb{R}^{d}$ on $\mathbb{R}^{d}$. Finally, the extremal log-Gaussian representation on the sphere will facilitate simulations of Brown-Resnick processes on the sphere.

Notation and conventions concerning max-stable processes are introduced in Chapter 1. which also revises structural results as well as some important examples of stationary max-stable processes that will figure throughout the text. While Chapter 1 and this very introduction are partially based on the manuscript [94] that has been submitted to the Bernoulli Journal and its precursory arXiv-version [93], Chapter 2 is mainly based on the manuscript [94]. Further, the arXiv-manuscript [93] has partially entered the considerations of Chapter 3 . Chapter 4 is based on joint work with Felix Ballani, where I am responsible for the main contributions. 

[...] it is necessary to march along paths opened by previous workers; acting otherwise, one runs too great a risk of creating a science without links with the rest of mathematics.

(Henri Lebesgue)

\section{Preliminaries}

We introduce our basic notions concerning max-stable processes and list the examples of max-stable processes that will be considered in this text. The material of this chapter is partially based on the manuscript 94] and its precursory arXiv-version 93.

\subsection{Max-stable processes}

A stochastic process $X=\left\{X_{t}\right\}_{t \in T}$ on an arbitrary index set $T$ is said to be maxstable if for each $n \in \mathbb{N}$ and independent copies $X^{(1)}, \ldots, X^{(n)}$ of $X$ the process of the maxima $\left\{\bigvee_{i=1}^{n} X^{(i)}\right\}_{t \in T}$ has the same law as $\left\{a_{n}(t) X_{t}+b_{n}(t)\right\}_{t \in T}$ for suitable norming functions $a_{n}(t)>0$ and $b_{n}(t) \in \mathbb{R}$ on $T$.

Marginal distributions In particular, the univariate marginal distributions of $X$ are max-stable. It is well-known that up to an affine transformation of the form $x \mapsto a x+b$ with $a>0$ and $b \in \mathbb{R}$ the non-degenerate univariate max-stable distributions are classified by belonging to one of the following three types (Fisher-Tippett theorem/Gnedenko's theorem):

$$
\begin{array}{rlrl}
\Phi_{\alpha}(x) & =\left\{\begin{array}{lll}
0 & x \leq 0 \\
\exp \left(-x^{-\alpha}\right) & x>0
\end{array}\right. & \alpha>0 & \text { (Fréchet) } \\
\Psi_{\alpha}(x) & =\left\{\begin{array}{lll}
\exp \left(-(-x)^{\alpha}\right) & x<0 \\
1 & x \geq 0
\end{array}\right. & \text { (Weibull) } \\
\Lambda(x) & =\exp \left(-e^{-x}\right) & \text { (Gumbel) }
\end{array}
$$


(cf. [31, 36]; see also [79, Proposition 0.3] or [16, Theorem 1.1.3] for the oneparameter representation due to von Mises and Jenkinson). Moreover, these marginal distributions can be transformed into each other by non-decreasing continuous transformations (cf. [23, p. 123]). Therefore and since we are interested in the dependence structure of max-stable processes, we shall primarily deal with max-stable processes $X$ that have standard Fréchet marginals as it is commonly done, i.e. $X$ satisfies

$$
\mathbb{P}\left(X_{t} \leq x\right)= \begin{cases}0 & x \leq 0 \\ e^{-1 / x} & x>0\end{cases}
$$

for $t \in T$. Here the sequence of normalizing functions will be $a_{n}(t)=n$ and $b_{n}(t)=0$ (cf. [23, p. 124]). Such standardized max-stable processes $X$ will be called simple max-stable processes.

Finite-dimensional distributions In order to describe the finite-dimensional distributions (f.d.d.) of a simple max-stable process $X$ on $T$, we shall fix some convenient notation: Let $M \subset T$ be some non-empty finite subset of $T$. By $\mathbb{R}^{M}$ (resp. [0, $]^{M}$ ) we denote the space of real-valued (resp. $[0, \infty]$-valued) functions on $M$. Elements of these spaces are denoted by $x=\left(x_{t}\right)_{t \in M}$ where $x_{t}=x(t)$. The standard scalar product is given through $\langle x, y\rangle=\sum_{t \in M} x_{t} y_{t}$. For a subset $L \subset M$ we write $\mathbf{1}_{L}$ for the indicator function of $L$ in $\mathbb{R}^{M}$ (resp. $[0, \infty]^{M}$ ), such that $\left\{\mathbf{1}_{\{t\}}\right\}_{t \in M}$ forms an orthonormal basis of $\mathbb{R}^{M}$. In this sense, the origin of $\mathbb{R}^{M}$ equals $\mathbf{1}_{\emptyset}$ being zero everywhere on $M$. Using this notation, we emphasize the fact that a multivariate distribution of a stochastic process is not any $|M|$-variate distribution, but it is bound to certain points (forming the set $M$ ) in the space $T$. Finally, we consider some reference norm $\|\cdot\|$ on $\mathbb{R}^{M}$ (not necessarily the one from the scalar product) and denote the positive unit sphere $S_{M}:=\left\{a \in[0, \infty)^{M}:\|a\|=1\right\}$.

The f.d.d. of a finite sample $\left\{X_{t}\right\}_{t \in M}$ of a simple max-stable process $X$ may be described by means of one of the following three quantities that are all equivalent to the knowledge of the respective $|M|$-variate simple max-stable distribution of $\left\{X_{t}\right\}_{t \in M}$ :

- the (finite-dimensional) spectral measure $H_{M}$ (cf. [17] or [79, Proposition 5.11.]), i.e. the finite Radon measure on $S_{M}$ such that for $x \in[0, \infty)^{M} \backslash\left\{\mathbf{1}_{\emptyset}\right\}$

$$
-\log \mathbb{P}\left(X_{t} \leq x_{t}, t \in M\right)=\int_{S_{M}}\left(\bigvee_{t \in M} \frac{a_{t}}{x_{t}}\right) H_{M}(\mathrm{~d} a),
$$

- the stable tail dependence function $\ell_{M}$ (cf. [4, p. 257]), i.e. the function on 
$[0, \infty)^{M}$ defined through

$$
\ell_{M}(x):=-\log \mathbb{P}\left(X_{t} \leq 1 / x_{t}, t \in M\right)=\int_{S_{M}}\left(\bigvee_{t \in M} a_{t} \cdot x_{t}\right) H_{M}(\mathrm{~d} a)
$$

- the (finite-dimensional) dependency set $\mathcal{K}_{M}$ (cf. [66]), i.e. the largest compact convex set $\mathcal{K}_{M} \subset[0, \infty)^{M}$ satisfying

$$
\ell_{M}(x)=\sup \left\{\langle x, y\rangle: y \in \mathcal{K}_{M}\right\} \quad \forall x \in[0, \infty)^{M} .
$$

In order to be a valid finite-dimensional spectral measure of a simple max-stable random vector $\left\{X_{t}\right\}_{t \in M}$, the only constraint that a finite Radon measure $H_{M}$ on $S_{M}$ has to satisfy is that $\int_{S_{M}} a_{t} H_{M}(\mathrm{~d} a)=1$ for all $t \in M$. This ensures standard Fréchet marginals. Moreover, up to this normalization to standard Fréchet marginals, it follows from [6] that stable tail dependence functions of multivariate simple maxstable distributions can be characterized as being sublinear, homogeneous and maxcompletely alternating, whereas dependency sets are max-zonoids. We address this matter in more detail in Proposition A.5.1. Equation (1.3) expresses that $\ell_{M}$ is the support function of $\mathcal{K}_{M}$ (cf. [87]).

Spectral representation Max-stable processes have a close connection to Poisson point processes. For theoretical background on Poisson point processes we refer to [13, 54, 79. In [15] de Haan shows that all (simple) max-stable processes $X=$ $\left\{X_{t}\right\}_{t \in T}$ that are either defined on a countable index set $T$ or defined on $T=\mathbb{R}$ and that are stochastically continuous may be represented as follows: There exists a finite measure $\nu$ on the Borel $\sigma$-algebra $\mathcal{B}([0,1])$ of $[0,1]$ and non-negative measurable functions $\left\{V_{t}\right\}_{t \in T}$ on $[0,1]$ (with $\int_{0}^{1} V_{t}(\omega) \nu(\mathrm{d} \omega)=1$ for each $t \in T$ ), such that

$$
\left\{X_{t}\right\}_{t \in T} \stackrel{\text { f.d.d. }}{=}\left\{\bigvee_{n=1}^{\infty} U_{n} V_{t}\left(\omega_{n}\right)\right\}_{t \in T}
$$

in the sense of finite-dimensional distributions (f.d.d.), where $\left\{\left(U_{n}, \omega_{n}\right)\right\}_{n=1}^{\infty}$ denotes an (enumerated) Poisson point process on $\mathbb{R}_{+} \times[0,1]$ with intensity $u^{-2} \mathrm{~d} u \times \nu(\mathrm{d} \omega)$. The normalization $\int_{0}^{1} V_{t}(\omega) \nu(\mathrm{d} \omega)=1$ is due to our choice of standard Fréchet marginals. For arbitrary unit Fréchet marginals with a different scale it is sufficient to require $\int_{0}^{1} V_{t}(\omega) \nu(\mathrm{d} \omega)<\infty$ instead.

In [92] Stoev and Taqqu introduce the slightly more general notion of an extremal stochastic integral by means of a random sup-measure $M_{\nu}$ with control measure $\nu$, which allows to involve arbitrary control measure spaces $(\Omega, \mathcal{A}, \nu)$ instead of consid- 
ering $([0,1], \mathcal{B}([0,1]), \nu)$ as above. Indeed, the r.h.s. of $(1.4)$ may be read as extremal stochastic integral

$$
\left\{X_{t}\right\}_{t \in T} \stackrel{\text { f.d.d. }}{=}\left\{\int_{\Omega}^{e} V_{t}(\omega) M_{\nu}(\mathrm{d} \omega)\right\}_{t \in T}
$$

with $\Omega=[0,1]$ and where $M_{\nu}$ denotes a random sup-measure with control measure $\nu$. We refer to [92] for a detailed explanation and to [96, p. 857] for an exploratory summary. For our purposes it will suffice to know that the f.d.d. of the process $X$ from (1.5) are given by

$$
-\log \mathbb{P}\left(X_{t} \leq x_{t}, t \in M\right)=\int_{\Omega}\left(\bigvee_{t \in M} \frac{V_{t}(\omega)}{x_{t}}\right) \nu(\mathrm{d} \omega)
$$

for $x \in[0, \infty)^{M} \backslash\left\{\mathbf{1}_{\emptyset}\right\}$ and any non-empty finite subset $M \subset T$.

Definition 1.1.1 (cf. [48, 96]). Let $(\Omega, \mathcal{A}, \nu)$ be a measure space and $V=\left\{V_{t}\right\}_{t \in T}$ non-negative measurable functions (with $\int_{\Omega} V_{t}(\omega) \nu(\mathrm{d} \omega)=1$ for each $t \in T$ ). We call $(\Omega, \mathcal{A}, \nu, V)$ a spectral representation of the (simple) max-stable process $X=$ $\left\{X_{t}\right\}_{t \in T}$, if 1.5 holds (or, equivalently, 1.6 holds for all non-empty subsets $M \subset$ $T)$. The functions $\left\{V_{t}\right\}_{t \in T}$ and the measure $\nu$ will be called spectral functions and spectral measure, respectively. In case $(\Omega, \mathcal{A}, \nu)$ is a probability space, the collection of spectral functions $V=\left\{V_{t}\right\}_{t \in T}$ themselves form a stochastic process that will be addressed as spectral process.

Of course, any stochastic process $X$ with a spectral representation 1.5 is (simple) max-stable. Conversely, it has been shown in [48, Theorem 1] that all (simple) maxstable processes allow for a spectral representation on some sufficiently rich measure space $(\Omega, \mathcal{A}, \nu)$. Moreover, given a (simple) max-stable process $X$ on a separable metric space $T$, the existence of a spectral representation $(\Omega, \mathcal{A}, \nu, V)$, where $(\Omega, \mathcal{A}, \nu)$ is a Lebesgue probability space (and the joint measurability of $(t, \omega) \mapsto V_{t}(\omega)$ in both variables $t$ and $\omega$ ) is guaranteed under mild conditions. This includes processes $X$ that have a measurable modification and especially stochastically continuous processes $X$ (cf. [48, Theorem 2], [96, Proposition 4.1.], [34, chapt. 3 sect. 3 Theorem 1]). Such max-stable processes $X$ are again representable in the form 1.4) (and not only (1.5)) with a spectral process $V=\left\{V_{t}\right\}_{t \in T}$. It is convenient in this case to interpret the expression $V\left(\omega_{n}\right)$ in 1.4 as a sequence $V^{(n)}$ of independent copies of a process $V=\left\{V_{t}\right\}_{t \in T}$ on $T$ that are independent of the Poisson point process $\left\{U_{n}\right\}_{n=1}^{\infty}$ on $\mathbb{R}_{+}$. However, we shall use other choices of $(\Omega, \mathcal{A}, \nu)$ when more appropriate for interpretations (as in the case of M3 processes, see Example 1.2.1). 


\subsection{Examples of max-stable processes}

The following processes $X=\left\{X_{t}\right\}_{t \in \mathbb{R}^{d}}$ on $\mathbb{R}^{d}$ are all max-stable and stationary, which means that the law of $\left\{X_{t+h}\right\}_{t \in \mathbb{R}^{d}}$ does not depend on $h \in \mathbb{R}^{d}$. We call a process $X=\left\{X_{t}\right\}_{t \in \mathbb{R}^{d}}$ on $\mathbb{R}^{d}$ stationary isotropic if the law of $\left\{X_{A t+h}\right\}_{t \in \mathbb{R}^{d}}$ does not depend on rigid motions $(A, h) \in \mathrm{SO}(d) \ltimes \mathbb{R}^{d}$. The subsequent examples have either been proposed already in previous literature or they constitute slight modifications of them. Here, they are all standardized to standard Fréchet marginals. Note that the stationarity of a spectral process $V$ is a sufficient, but not a necessary condition for $X$ being stationary (cf. [52, 67] and Proposition A.3.1).

Example 1.2.1. (Mixed Moving Maxima processes (M3)) Slightly different notions are used in the literature (cf. [51, 83, 90, 91, 92]). We consider the following normalized version: A Mixed Moving Maxima process (M3 process) is a simple max-stable process $X$ on $\mathbb{R}^{d}$ with the following spectral representation $(\Omega, \mathcal{A}, \nu, V)$ :

- $(\Omega, \mathcal{A}, \nu)=\left(\mathbb{F} \times \mathbb{R}^{d}, \mathcal{F} \otimes \mathcal{B}\left(\mathbb{R}^{d}\right), \mu \otimes \mathrm{d} z\right)$, where $\left(\mathbb{R}^{d}, \mathcal{B}\left(\mathbb{R}^{d}\right), \mathrm{d} z\right)$ denotes the Lebesgue measure on the Borel $\sigma$-algebra of $\mathbb{R}^{d}$ and where $(\mathbb{F}, \mathcal{F}, \mu)$ denotes a measure space of $[0, \infty]$-valued measurable functions on $\mathbb{R}^{d}$, such that the assignment $\mathbb{F} \times \mathbb{R}^{d} \ni(f, z) \mapsto f(z)$ is measurable and such that

$$
\int_{\mathbb{F}}\left(\int_{\mathbb{R}^{d}} f(z) \mathrm{d} z\right) \mu(\mathrm{d} f)=1
$$

- $V_{t}((f, z))=f(t-z)$ for $t \in \mathbb{R}^{d}$.

It can be easily checked that the process $X$ is stationary. In case the measure $\mu$ is a point mass (and $f$ with $\|f\|_{L^{1}}=1$ is deterministic), the process $X$ is called a Moving Maxima process. If the measure $\mu$ is a probability measure, with (1.4) in mind the M3 process $X$ is sometimes interpreted as a process of random storms $f$ of a certain severity $U$ centered around $z$. As in [27, 70] we will address the involved functions $f \in \mathbb{F}$ as (random) shape functions.

Example 1.2.2. (Extremal Gaussian processes and extremal binary Gaussian processes (EG and EBG)) Here we relate to [83, Theorem 2]. Let $Z=\left\{Z_{t}\right\}_{t \in \mathbb{R}^{d}}$ be a stationary Gaussian process whose marginals follow a standard normal distribution. The correlation function of $Z$ will be denoted by $\rho(t)$ and is simply $\rho(t)=\mathbb{E}\left(Z_{t} Z_{o}\right)$ due to the standard normal marginals. Based on $Z$, we call the process $X$ defined through the spectral process

$$
V_{t}=\sqrt{2 \pi} \cdot\left(Z_{t}\right)_{+} \quad t \in \mathbb{R}^{d}
$$


extremal Gaussian process (EG process) (where $z_{+}=\max (z, 0)$ ). Secondly, we call the process $X$ with spectral process

$$
V_{t}=2 \cdot \mathbb{1}_{\left\{Z_{t}>0\right\}} \quad t \in \mathbb{R}^{d}
$$

extremal binary Gaussian process (EBG process). Again it is easy to check that EG processes and EBG processes are stationary and simple max-stable. An advantage of such processes is that they can be simulated directly from Gaussian processes, using only a correlation function as parameter. Of course, in both cases the law of $X$ depends on the correlation function $\rho$ only.

Example 1.2.3. (Brown-Resnick processes (BR)) In [52] Brown-Resnick processes $\eta=\{\eta(t)\}_{t \in \mathbb{R}^{d}}$ are introduced with standard Gumbel marginals. Here, we shall primarily consider $X_{t}=\exp (\eta(t))$, which amounts to standard Fréchet marginals instead: Let $\left\{W_{t}\right\}_{t \in \mathbb{R}^{d}}$ be a Gaussian process with stationary increments (meaning that the law of $\left\{W_{t+h}-W_{h}\right\}_{t \in \mathbb{R}^{d}}$ does not depend on $h \in \mathbb{R}^{d}$ ) and variance $\sigma^{2}(t)=\operatorname{Var}\left(W_{t}\right)$. Then we call the process $X$ defined through the spectral process

$$
V_{t}=\exp \left(W_{t}-\frac{\sigma^{2}(t)}{2}\right) \quad t \in \mathbb{R}^{d}
$$

Brown-Resnick process (BR process). The law of $X$ is stationary, simple max-stable and depends on the variogram $\gamma(t)=\mathbb{E}\left(W_{t}-W_{o}\right)^{2}$ only. It is neither obvious that $X$ will be stationary nor that the law of $X$ depends only on the variogram. We refer to [52, Theorem 2] (rephrased in Theorem A.3.2) for both statements. These processes are particularly attractive for modelling as they occur as natural limits for maxima of Gaussian processes ([52, Theorem 20]) and allow for a wide flexibility in their dependence structure using only the variogram as parameter.

Remark 1.2.4. The processes above exhibit different behaviour towards long-range dependence. While M3 processes are shown to be mixing (on $\mathbb{R}$ [91]; or generated by a dissipative flow [51]), EG and EBG processes feature long-range dependence (generated by a positive recurrent flow, cf. [51, p. 419]). Brown-Resnick processes entail both behaviours depending on the variogram. If the variogram tends to $\infty$ fast enough, Brown-Resnick processes may even be representable as an M3 process (cf. [52, Theorem 14]). See also [97] for ergodic properties of max-stable processes defined on $\mathbb{R}^{d}$.

Additionally, we shall consider a special subclass of M3 processes based on indicator functions of Poisson polytopes, which is a mixed and slightly modified version of a max-stable process introduced in [55] and, secondly, a "variance-mixed" version of Brown-Resnick processes: 
Example 1.2.5. (Mixed Poisson storm processes (MPS)) Here, we consider a mixed version of the Poisson storm process introduced in [55]. Before we define the process, let us make some preliminary considerations (with terminology from stochastic geometry based on [88]).

We denote $\kappa_{d}:=\nu_{d}\left(B_{1}^{d}(o)\right)=\pi^{d / 2} / \Gamma(1+d / 2)$ the volume of the $d$-dimensional unit ball. If $C$ is the typical cell of a stationary isotropic Poisson hyperplane mosaic of intensity 1 (cf. [88, pp. 497 and p. 126] for the intensity) and $\beta>0$, then $1 / \beta \cdot C$ is distributed like the typical cell corresponding to the intensity $\beta$ and has expected volume

$$
\mathbb{E}\left(\nu_{d}\left(\frac{1}{\beta} \cdot C\right)\right)=\frac{d^{d} \kappa_{d}^{d-1}}{\kappa_{d-1}^{d}} \cdot \frac{1}{\beta^{d}}=: \frac{1}{\mu_{d}(\beta)}
$$

(cf. [88, (10.4) and (10.4.6)]). Note that our notion of intensity $\beta$ is based on [88, p. 126] and corresponds to the choice $\lambda=\beta \kappa_{d-1} /\left(d \kappa_{d}\right)$ with $\lambda$ as in [55, p. 420].

Now, let $\beta \in(0, \infty)$ be a random variable distributed according to a distribution function $F$ on $(0, \infty)$ (with $F(0+)=0$ ). Let $C$ be the typical cell of a stationary isotropic Poisson hyperplane mosaic of intensity 1 that is independent of $\beta$ and set

$$
f(t):=\mu_{d}(\beta) \mathbb{1}_{\frac{1}{\beta} \cdot C}(t) \quad t \in \mathbb{R}^{d}
$$

where $\mathbb{1}_{\frac{1}{\beta} C}$ denotes the indicator function of $\frac{1}{\beta} C$. Conditioning on $\beta$, one sees that, indeed, $f$ satisfies (1.7) and thus, defines an M3 process with standard Fréchet marginals, which is stationary isotropic. We call this process Mixed Poisson storm process with intensity mixing distribution $F$. We shall see that the considered characteristics of these processes admit nice expressions in view of a geometric interpretation.

Example 1.2.6. (Variance-mixed Brown-Resnick processes (VBR)) Finally, let us consider a mixture of Brown-Resnick processes with respect to the variance of the involved Gaussian process. As in the construction of Brown-Resnick processes let $\left\{W_{t}\right\}_{t \in \mathbb{R}^{d}}$ be a Gaussian process with stationary increments and variance $\sigma^{2}(t)$. Additionally, let $S$ be an independent random variable on $(0, \infty)$ with distribution function $G$ (with $G(0+)=0$ ). Then we call the process $X$ with spectral process

$$
V_{t}=\exp \left(S W_{t}-\frac{S^{2}}{2} \sigma^{2}(t)\right) \quad t \in \mathbb{R}^{d}
$$

variance-mixed Brown-Resnick process with variance mixing distribution $G$. 
The law of $X$ is stationary, simple max-stable and depends on the variogram $\gamma(t)=$ $\mathbb{E}\left(W_{t}-W_{o}\right)^{2}$ and the distribution function $G$ only (cf. Corollary A.3.3). A similar construction can be found in [25], where the Brown-Resnick process is mixed in its scale instead. This yields in fact the same class of processes in the most prominent example when $W_{t}$ is a fractal Brownian motion and thus, self-similar. 
Our minds are finite, and yet even in these circumstances of finitude we are surrounded by possibilities that are infinite, and the purpose of life is to grasp as much as we can out of that infinitude.

(Alfred North Whitehead)

\section{Max-stable processes parametrized by their extremal coefficients}

This chapter is primarily based on the manuscript [94] and its precursory arXivversion 93 .

\subsection{Extremal coefficient functions}

Given a simple max-stable process $X=\left\{X_{t}\right\}_{t \in T}$ on an arbitrary index set $T$, we may assign to each non-empty finite subset $A \subset T$ the extremal coefficient $\theta(A)$ (cf. [85, 90]), that is

$$
\theta(A):=\lim _{x \rightarrow \infty} \frac{\log \mathbb{P}\left(\bigvee_{t \in A} X_{t} \leq x\right)}{\log \mathbb{P}\left(X_{t} \leq x\right)}=\int_{S_{M}}\left(\bigvee_{t \in A} a_{t}\right) H_{M}(\mathrm{~d} a)=\ell_{M}\left(\mathbf{1}_{A}\right),
$$

for $A \subset M$, where $H_{M}$ and $\ell_{M}$ denote the (finite-dimensional) spectral measure (1.1) and the stable tail dependence function 1.2 , respectively.

Indeed, the expression $\log \mathbb{P}\left(\bigvee_{t \in A} X_{t} \leq x\right) / \log \mathbb{P}\left(X_{t} \leq x\right)$ does not depend on $x$ and equals the r.h.s. Observe that $\theta(A)$ takes values in the interval $[1,|A|]$, where the value 1 corresponds to full dependence of the random variables $\left\{X_{t}\right\}_{t \in A}$ and the value $|A|$ (number of elements in $A$ ) corresponds to independence. Roughly speaking, the extremal coefficient $\theta(A)$ detects the effective number of independent variables among the random variables $\left\{X_{t}\right\}_{t \in A}$. It is coherent to set $\theta(\emptyset):=0$ to obtain a function $\theta$ on $\mathcal{F}(T)$, the set of finite subsets of $T$. We call the function

$$
\theta: \mathcal{F}(T) \rightarrow[0, \infty)
$$

extremal coefficient function (ECF) of the process $X$. 
The set of all ECFs of simple max-stable processes on a set $T$ will be denoted by

$$
\Theta(T):=\left\{\theta: \mathcal{F}(T) \rightarrow[0, \infty): \begin{array}{l}
\theta \text { is an ECF of a simple } \\
\text { max-stable process on } T .
\end{array}\right\} .
$$

Example 2.1.1. The simplest ECFs are the functions $\theta(A)=|A|$ corresponding to a process of independent random variables, and the indicator function $\theta(A)=\mathbb{1}_{A \neq \emptyset}$ corresponding to a process of identical random variables.

Rather sophisticated examples of ECFs can be obtained using the spectral representations $(\Omega, \mathcal{A}, \nu, V)$ of processes $X$ (cf. (1.6)). In these terms the ECF $\theta$ of $X$ is given by

$$
\theta(A)=\int_{\Omega}\left(\bigvee_{t \in A} V_{t}(\omega)\right) \nu(\mathrm{d} \omega)
$$

for $A \in \mathcal{F}(T) \backslash\{\emptyset\}$ and $\theta(\emptyset)=0$.

Example 2.1.2 (Mixed Moving Maxima processes (M3)). Because of 2.3 the ECF $\theta$ of an M3 process $X$ as in Example 1.2.1 can be computed as

$$
\theta(A)=\int_{\mathbb{F}} \int_{\mathbb{R}^{d}}\left(\bigvee_{t \in A} f(t-z)\right) \mathrm{d} z \mu(\mathrm{d} f)
$$

for $A \in \mathcal{F}\left(\mathbb{R}^{d}\right) \backslash\{\emptyset\}$ and $\theta(\emptyset)=0$. In case $\mu$ is a point mass at an indicator function $f$, the bivariate coefficient $\theta(\{s, t\})$ will be given by $\theta(\{s, t\})=2-f * \check{f}(s-t)$, where $f * \check{f}$ means the convolution of $f$ with $\check{f}$ and $\check{f}(t)=f(-t)$.

Example 2.1.3 (Brown-Resnick processes). Because of (2.3) the ECF $\theta$ of a BrownResnick process $X$ as in Example 1.2.3 is

$$
\theta(A)=\mathbb{E}_{W} \exp \left(\bigvee_{t \in A} W_{t}-\sigma^{2}(t) / 2\right)
$$

for $A \in \mathcal{F}\left(\mathbb{R}^{d}\right) \backslash\{\emptyset\}$ and $\theta(\emptyset)=0$. Since the f.d.d. of $X$ only depend on the variogram $\gamma$, the extremal coefficient $\theta(A)$ will also depend only on the values $\{\gamma(s-t)\}_{s, t \in A}$. In particular, we have $\theta(\{s, t\})=1+\operatorname{erf}(\sqrt{\gamma(s-t) / 8})$ for the bivariate coefficient $\theta(\{s, t\})$, where $\operatorname{erf}(x)=2 / \sqrt{\pi} \int_{0}^{x} e^{-t^{2}} d t$ denotes the error function (cf. [52, Remark 25]). In case the variogram equals $\gamma(z)=\lambda\|z\|_{2}^{2}$ for some $\lambda>0$, explicit formulas for multivariate coefficients of higher orders up to $d+1$ can be found in [33]. 
Example 2.1.4 (Mixed Poisson storm process). In this example it can be seen explicitly, how $\theta$ depends on the geometry of $A$. We consider the Mixed Poisson storm process $X$ with intensity mixing distribution $F$ as in Example 1.2.5. Conditioning on the intensity $\beta$, we see that $\theta(A)=\int \theta_{\beta}(A) \mathrm{d} F(\beta)$. Following [55, Proposition 4] we compute in our parametrization (with $K=A \subset \mathbb{R}^{d}, \mu=\mu_{d}(\beta), \omega_{d}=\kappa_{d}$ and $\left.\lambda=\beta \kappa_{d-1} /\left(d \kappa_{d}\right)\right)$ that $\theta_{\beta}(A)=\sum_{\emptyset \neq L \subset A}(-1)^{|L|-1} \chi_{\beta}(L)$ with

$$
\begin{aligned}
\chi_{\beta}(L) & =\mathbb{E}_{C}\left[\mu_{d}(\beta) \cdot \int_{\mathbb{R}^{d}} \prod_{t \in L} \mathbb{1}_{\frac{1}{\beta} \cdot C}(z-t) \mathrm{d} z\right]=\frac{\mathbb{E}_{C}\left[\nu_{d}\left(\bigcap_{t \in L}\left(\frac{C}{\beta}+t\right)\right)\right]}{\mathbb{E}_{C}\left[\nu_{d}\left(\frac{C}{\beta}\right)\right]} \\
& =\mathbb{P}_{C}\left[\bigcap_{t \in L}\left(\frac{C}{\beta}+t\right) \neq \emptyset\right]=\exp (-\beta \cdot b(\operatorname{conv}(L))),
\end{aligned}
$$

where $b(\operatorname{conv}(L))$ denotes the mean width of the convex hull of $L$ (cf. [88, p. 601 (14.7)]). Summarizing, we obtain

$$
\theta(A)=\sum_{\emptyset \neq L \subset A}(-1)^{|L|-1} \mathcal{L}(F)(b(\operatorname{conv}(L)))
$$

for the ECF of the Mixed Poisson storm process with mixing distribution $F$. Here $\mathcal{L}(F)(x)=\int_{(0, \infty)} \exp (-x t) \mathrm{d} F(t)$ denotes the Laplace transform of $F$, which is evaluated at the mean width $b(\operatorname{conv}(L))$ of the convex hull of $L$.

\subsection{A consistent max-linear model}

A multivariate simple max-stable distribution is called max-linear (or spectrally discrete) if it arises as the distribution of a random vector $X$ of the following form

$$
X_{i}=\bigvee_{j=1}^{q} a_{i j} Z_{j} \quad i=1, \ldots, p
$$

where $Z=\left\{Z_{j}\right\}_{j=1}^{q}$ is a vector of i.i.d. unit Fréchet random variables and where $\left\{a_{i j}\right\}_{p \times q}$ is a matrix of non-negative entries with $\sum_{j=1}^{q} a_{i j}=1$ for each row $i=$ $1, \ldots, p$. This is equivalent to requiring the spectral measure $H_{M}$ from (1.1) for $M=\{1, \ldots, i, \ldots, p\}$ to be the following discrete measure on $S_{M}$

$$
H_{M}=\sum_{j=1}^{q}\left\|a_{j}\right\| \delta_{a_{j} /\left\|a_{j}\right\|}
$$


where $a_{j}$ denote the column vectors of the matrix $\left\{a_{i j}\right\}_{p \times q}$. Conversely, any discrete spectral measure of a simple max-stable random vector gives rise to such a matrix. Surely, the ECF of such a random vector $X=\left\{X_{i}\right\}_{i \in M}$ is

$$
\theta(A)=\sum_{j=1}^{q} \bigvee_{i \in A} a_{i j}
$$

for $\emptyset \neq A \subset M$ and $\theta(\emptyset)=0$ (cf. (2.1)).

In [85. Schlather and Tawn introduce a max-linear model for $X^{*}=\left\{X_{i}^{*}\right\}_{i \in M}$ where the column index $j$ ranges over all non-empty subsets $L$ of $M$ and where non-negative coefficients $\tau_{L}$ are given for each column $\emptyset \neq L \subset M$, more precisely

$$
X_{i}^{*}=\bigvee_{\emptyset \neq L \subset M} a_{i, L} Z_{L} \quad i \in M \quad \text { with } \quad a_{i, L}=\tau_{L} \mathbb{1}_{i \in L}
$$

which is equivalent to

$$
X_{i}^{*}=\bigvee_{i \in L \subset M} \tau_{L} Z_{L} \quad i \in M
$$

The model (2.6) is simple if and only if $\sum_{\emptyset \neq L \subset M} a_{i L}=\sum_{L \subset M: i \in L} \tau_{L}=1$ for each $i \in M$. It follows from (2.5) that the ECF of model (2.6) is

$$
\theta(A)=\sum_{L \subset M: A \cap L \neq \emptyset} \tau_{L}
$$

for $\emptyset \neq A \subset M$ and $\theta(\emptyset)=0$. Now, the interesting aspect of this model (2.6) with given coefficients $\tau_{L}$ is that such models are in 1-1 correspondence with ECFs $\theta$ on the finite set $M$ (cf. [85, Theorem 3 and 4]). Alongside, this leads to a set of inequalities which fully characterizes the set of ECFs $\Theta(M)$ for finite sets $M$ (cf. [85. Corollary 5]). In [66, Corollary 1] Molchanov offers an alternative proof for these inequalities and notices that they are equivalent to a property called complete alternation (see below).

As we seek a spatial generalization of these results, let us consider a max-stable process $X^{*}=\left\{X_{t}^{*}\right\}_{t \in T}$ on an arbitrary index set $T$, whose f.d.d. for a finite set $M$ are precisely of the above form (2.6), where the coefficients $\tau_{L}$ now additionally depend on $M$. That means we set the spectral measure $H_{M}^{*}$ of the random vector $\left\{X_{t}^{*}\right\}_{t \in M}$

$$
H_{M}^{*}:=\sum_{\emptyset \neq L \subset M} \tau_{L}^{M}\left\|\mathbf{1}_{L}\right\| \delta_{\mathbf{1}_{L} /\left\|\mathbf{1}_{L}\right\|}
$$




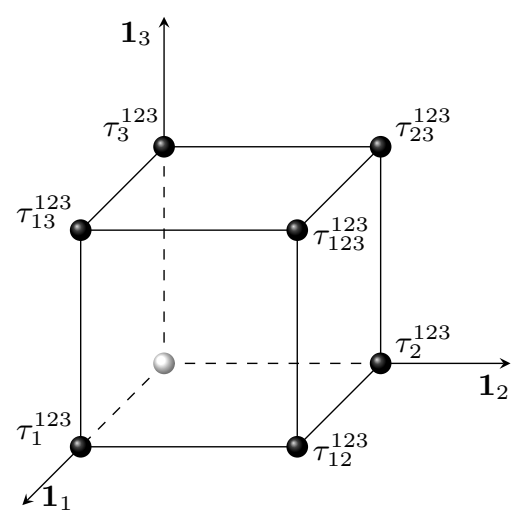

Figure 2.1.: Spectral measure representation of $\left\{X_{t}^{*}\right\}_{t \in M}$ for $M=\{1,2,3\}$ if we choose the reference norm on $\mathbb{R}^{M}$ to be the maximum norm $\|\cdot\|_{\infty}$. In this case the spectral measure simplifies to a sum of weighted point masses on the vertices of a cube: $H_{M}^{*}=\sum_{\emptyset \neq L \subset M} \tau_{L}^{M} \delta_{1_{L}}$.

such that the f.d.d. of the process $X^{*}$ are given by (cf. (1.1))

$$
-\log \mathbb{P}\left(X_{t}^{*} \leq x_{t}, t \in M\right)=\sum_{\emptyset \neq L \subset M} \tau_{L}^{M} \bigvee_{t \in L} \frac{1}{x_{t}}
$$

Here $M$ ranges over all non-empty finite subsets of $T$, which we express as $M \in$ $\mathcal{F}(T) \backslash\{\emptyset\}$. Figure 2.1 illustrates this spectral measure for a trivariate distribution where $M=\{1,2,3\}$ in case the reference norm is the maximum norm.

Lemma 2.2.1. Let $T$ be an arbitrary set and let coefficients $\tau_{L}^{M}$ be given for $M \in$ $\mathcal{F}(T) \backslash\{\emptyset\}$ and $L \in \mathcal{F}(M) \backslash\{\emptyset\}$, such that

(i) $\tau_{L}^{M} \geq 0$ for all $M \in \mathcal{F}(T) \backslash\{\emptyset\}$ and $L \in \mathcal{F}(M) \backslash\{\emptyset\}$,

(ii) $\tau_{L}^{M}=\tau_{L}^{M \cup\{t\}}+\tau_{L \cup\{t\}}^{M \cup\{t\}}$ for all $M \in \mathcal{F}(T) \backslash\{\emptyset\}$ and $L \in \mathcal{F}(M) \backslash\{\emptyset\}$ and $t \in T \backslash M$,

(iii) $\tau_{\{t\}}^{\{t\}}=1$ for all $t \in T$.

Then the spectral measures $\left\{H_{M}^{*}\right\}_{M \in \mathcal{F}(T) \backslash\{\emptyset\}}$ from (2.7) define a simple max-stable process $X^{*}=\left\{X_{t}^{*}\right\}_{t \in T}$ on $T$ with f.d.d. as in (2.8).

Proof. Condition (ii) ensures that each spectral measure $H_{M}^{*}$ defines a max-stable distribution with Fréchet marginals. Subsequently, condition (ii) ensures consistency of these distributions (i.e. the conditions for Kolmogorov's extension theorem are satisfied). Hence the spectral measures $H_{M}^{*}$ define a max-stable process $X^{*}$ on $T$. Finally, condition (iii) ensures that the process $X^{*}$ has unit Fréchet marginals. 
Remark 2.2.2. Note that condition (ii) is equivalent to

$$
\tau_{K}^{A}=\sum_{J \subset M \backslash A} \tau_{K \cup J}^{M} \quad \forall M \in \mathcal{F}(T) \backslash\{\emptyset\}, \emptyset \neq K \subset A \subset M .
$$

\subsection{Negative definiteness of ECFs}

For the following characterization of the set of ECFs $\Theta(T)$ we use the fact that $\mathcal{F}(T)$, the set of finite subsets of $T$, forms an abelian semigroup with respect to the union operation $\cup$ and with neutral element the empty set $\emptyset$. The semigroup $(\mathcal{F}(T), \cup, \emptyset)$ is idempotent (meaning $A \cup A=A$ for $A \in \mathcal{F}(T)$ ), partially ordered by inclusion of sets and generated by the singletons $\{\{t\}\}_{t \in T}$. Section A.1 summarizes several facts from harmonic analysis for such semigroups based on [5] and [65]. The following notation is adopted from there. For a function $f: \mathcal{F}(T) \rightarrow \mathbb{R}$ and elements $K, L \in \mathcal{F}(T)$ we set

$$
\left(\Delta_{K} f\right)(L):=f(L)-f(L \cup K)
$$

Note that the operators $\Delta_{K_{1}}$ and $\Delta_{K_{2}}$ commute with each other and that $\Delta_{K} \Delta_{K}=$ $\Delta_{K}$ (since $\mathcal{F}(T)$ is idempotent).

Definition 2.3.1 (negative definiteness, complete alternation).

A function $\psi: \mathcal{F}(T) \rightarrow \mathbb{R}$ is called negative definite (in the semigroup sense) on $\mathcal{F}(T)$ if for all $n \geq 2,\left\{K_{1}, \ldots, K_{n}\right\} \subset \mathcal{F}(T)$ and $\left\{a_{1}, \ldots, a_{n}\right\} \subset \mathbb{R}$ with $\sum_{j=1}^{n} a_{j}=0$

$$
\sum_{j=1}^{n} \sum_{k=1}^{n} a_{j} a_{k} \psi\left(K_{j} \cup K_{k}\right) \leq 0
$$

A function $\psi: \mathcal{F}(T) \rightarrow \mathbb{R}$ is called completely alternating on $\mathcal{F}(T)$ if for all $n \geq 1$, $\left\{K_{1}, \ldots, K_{n}\right\} \subset \mathcal{F}(T)$ and $K \in \mathcal{F}(T)$

$$
\left(\Delta_{K_{1}} \Delta_{K_{2}} \ldots \Delta_{K_{n}} \psi\right)(K)=\sum_{I \subset\{1, \ldots, n\}}(-1)^{|I|} \psi\left(K \cup \bigcup_{i \in I} K_{i}\right) \leq 0 .
$$

Because the semigroup $(\mathcal{F}(T), \cup, \emptyset)$ is idempotent, these two terms coincide. That means $\psi: \mathcal{F}(T) \rightarrow \mathbb{R}$ is completely alternating if and only if $\psi$ is negative definite (in the semigroup sense), cf. [5, 4.4.16] or (A.2). In fact, the condition (2.10) can be slightly weakened. 
Lemma 2.3.2. A function $\psi: \mathcal{F}(T) \rightarrow \mathbb{R}$ is negative definite (completely alternating) on $\mathcal{F}(T)$ if and only if for all $\emptyset \neq L \in \mathcal{F}(T)$ and $K \in \mathcal{F}(T)$ with $K \cap L=\emptyset$

$$
\sum_{I \subset L}(-1)^{|I|+1} \psi(K \cup I) \geq 0
$$

Proof. Because the semigroup $\mathcal{F}(T)$ is generated by the singletons $\{t\}$ for $t \in T$, it suffices already to require (2.10) only for $K_{i}=\left\{t_{i}\right\}$ for $t_{i} \in T(i=1, \ldots, n)$, cf. [5. 4.6.6.] or Lemma A.1.7. Since $\Delta_{\{t\}} \Delta_{\{t\}}=\Delta_{\{t\}}$ it suffices to choose pairwise different $t_{i}(i=1, \ldots, n)$. Hence $\psi$ is completely alternating on $\mathcal{F}(T)$ if and only if for all $\emptyset \neq L \in \mathcal{F}(T)$ and $K \in \mathcal{F}(T)$ the inequality 2.11 holds. Finally, the expression on the 1.h.s. of (2.11) equals automatically 0 if $K \cap L \neq \emptyset$.

For finite sets $M$ (instead of arbitrary $T$ ), negative definiteness (complete alternation) can be formulated by bounding the value $\psi(M)$ by lower order values $\psi(L)$ for $L \subset M$. The following lemma shows the connection to [85, (12)].

Lemma 2.3.3. Let $M$ be a non-empty finite set. Then $\psi: \mathcal{F}(M) \rightarrow \mathbb{R}$ is negative definite (completely alternating) on $\mathcal{F}(M)$ if and only if

$$
\sum_{I \subset L}(-1)^{|I|+1} \psi((M \backslash L) \cup I) \geq 0 \quad \forall \emptyset \neq L \subset M,
$$

which is equivalent to

$$
\bigvee_{\substack{L \subset M \\|L| \text { odd } \\ I \neq L}} \sum_{\substack{I \subset L \\ I \neq L}}(-1)^{|I|} \psi((M \backslash L) \cup I) \leq \psi(M) \leq \bigwedge_{\substack{\emptyset \neq L \subset M \\|L| \text { even } \\ I \neq L}} \sum_{\substack{I \subset L \\ I \neq L}}(-1)^{|I|+1} \psi((M \backslash L) \cup I)
$$

Proof. Because of

$$
\sum_{I \subset L}(-1)^{|I|+1} \psi(K \cup I)=\sum_{J \subset M \backslash(K \cup L)}\left(\sum_{I \subset L \cup J}(-1)^{|I|+1} \psi((M \backslash(L \cup J)) \cup I)\right)
$$

for $K, L \subset M$ with $K \cap L=\emptyset$ (cf. Lemma A.5.3), it suffices to check 2.11) for $K=M \backslash L$. Summarizing the cases where $|L|$ is odd and where $|L|$ is even yields the second equivalence.

Example 2.3.4 ([65], p. 52). Let $Y=\left\{Y_{t}\right\}_{t \in T}$ be a stochastic process with values in $\{0,1\}$ and let the function $C: \mathcal{F}(T) \rightarrow \mathbb{R}$ be given by $C(\emptyset)=0$ and

$$
C(A)=\mathbb{P}\left(\exists t \in A \text { such that } Y_{t}=1\right)=\mathbb{P}\left(\bigvee_{t \in A} Y_{t}=1\right)
$$


Then $C$ is negative definite (completely alternating) on $\mathcal{F}(T)$. The function $C$ is called the capacity functional of $Y$. Conversely, if $C: \mathcal{F}(T) \rightarrow[0,1]$ is completely alternating with $C(\emptyset)=0$, then $C$ determines the f.d.d. of a stochastic process $Y=\left\{Y_{t}\right\}_{t \in T}$ with values in $\{0,1\}$, whose capacity functional equals $C$.

Now, we can characterize the set $\Theta(T)$ of possible ECFs on $\mathcal{F}(T)$ and define a corresponding max-linear process $X^{*}$ as follows.

Theorem 2.3.5. a) The function $\theta: \mathcal{F}(T) \rightarrow \mathbb{R}$ is the ECF of a simple max-stable process on $T$ if and only if the following conditions are satisfied:

(i) $\theta$ is negative definite (completely alternating) on $\mathcal{F}(T)$,

(ii) $\theta(\emptyset)=0$,

(iii) $\theta(\{t\})=1$ for all $t \in T$.

b) If these conditions are satisfied, the following choice of coefficients

$$
\begin{aligned}
\tau_{L}^{M}:=-\Delta_{\left\{t_{1}\right\}} \ldots \Delta_{\left\{t_{l}\right\}} \theta(M \backslash L) & =\sum_{I \subset L}(-1)^{|I|+1} \theta((M \backslash L) \cup I) \\
\forall M & \in \mathcal{F}(T) \backslash\{\emptyset\}, \emptyset \neq L=\left\{t_{1}, \ldots, t_{l}\right\} \subset M
\end{aligned}
$$

for model (2.7) defines a simple max-stable process $X^{*}$ on $T$ which realizes $\theta$ as its own ECF $\theta^{*}$.

Proof. If $\theta$ is an ECF of a simple max-stable process $X$ on $T$, then necessarily $\theta(\emptyset)=0$ and $\theta(\{t\})=1$ for all $t \in T$ (cf. (2.1)). Further, it is an application of l'Hôpitals rule (apply Lemma A.5.4 to the choice $p(x):=\mathbb{P}\left(X_{t} \leq x\right)$ and $\alpha=\theta(A)$ ) that for $A \subset \mathcal{F}(T) \backslash\{\emptyset\}$

$$
\begin{aligned}
\theta(A) & =\lim _{x \rightarrow \infty} \frac{-\log \mathbb{P}\left(\bigvee_{t \in A} X_{t} \leq x\right)}{-\log \mathbb{P}\left(X_{t} \leq x\right)}=\lim _{x \rightarrow \infty} \frac{1-\mathbb{P}\left(\bigvee_{t \in A} X_{t} \leq x\right)}{1-\mathbb{P}\left(X_{t} \leq x\right)} \\
& =\lim _{x \rightarrow \infty} \frac{\mathbb{P}\left(\exists t \in A \text { such that } X_{t} \geq x\right)}{\mathbb{P}\left(X_{t} \geq x\right)}=\lim _{x \rightarrow \infty} \frac{C^{(x)}(A)}{p^{(x)}},
\end{aligned}
$$

where $C^{(x)}$ denotes the capacity functional for the binary process $Y_{t}=\mathbb{1}_{X_{t} \geq x}$ and $p^{(x)}=\mathbb{E} Y_{t}=1-e^{-1 / x}$. Since negative definiteness respects scaling and pointwise limits, negative definiteness of $\theta$ follows from Example 2.3.4. This shows the necessity of (i),(ii),(iii).

Conversely, let $\theta: \mathcal{F}(T) \rightarrow \mathbb{R}$ be a function satisfying conditions (i),(ii),(iii) and let the coefficients $\tau_{L}^{M}$ be given as above. We need to check that they fulfill the (in)equalities from Lemma 2.2.1. Indeed we have: 
- The inequalities $\tau_{L}^{M}=-\Delta_{\left\{t_{1}\right\}} \ldots \Delta_{\left\{t_{l}\right\}} \theta(M \backslash L) \geq 0$ follow directly from the complete alternation of $\theta$.

- From the definition of $\Delta_{\{t\}}$ we observe

$$
\begin{aligned}
\tau_{L \cup\{t\}}^{M \cup\{t\}} & =-\Delta_{\{t\}} \Delta_{\left\{t_{1}\right\}} \ldots \Delta_{\left\{t_{l}\right\}} \theta((M \cup\{t\}) \backslash(L \cup\{t\})) \\
& =-\Delta_{\left\{t_{1}\right\}} \ldots \Delta_{\left\{t_{l}\right\}} \theta(M \backslash L)+\Delta_{\left\{t_{1}\right\}} \ldots \Delta_{\left\{t_{l}\right\}} \theta(M \cup\{t\} \backslash L) \\
& =\tau_{L}^{M}-\tau_{L}^{M \cup\{t\}} .
\end{aligned}
$$

- For $t \in T$ we have $\tau_{\{t\}}^{\{t\}}=\theta(\{t\})=1$ because of (iii).

Thus, the coefficients $\tau_{L}^{M}$ define a simple max-stable process $X^{*}$ on $T$ as given by model (2.7). Finally, we compute the ECF $\theta^{*}$ of $X^{*}$ and see that it coincides with $\theta$ : For the empty set we have $\theta^{*}(\emptyset)=0=\theta(\emptyset)$ because of (ii); otherwise we compute for $A \subset \mathcal{F}(T) \backslash\{\emptyset\}$ that

$$
\begin{aligned}
& \theta^{*}(A) \text { 2.1, 2.77 } \sum_{\emptyset \neq L \subset A} \tau_{L}^{A}=\sum_{\emptyset \neq L \subset A} \sum_{I \subset L}(-1)^{|I|+1} \theta((A \backslash L) \cup I)
\end{aligned}
$$

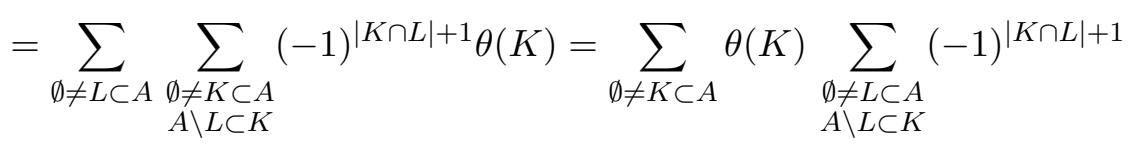

$$
\begin{aligned}
& =\sum_{\emptyset \neq K \subset A} \theta(K)\left(-\left(-\mathbb{1}_{K=A}\right)\right)=\theta(A) .
\end{aligned}
$$

This shows sufficiency of (i),(ii),(iii) and part b).

Theorem 2.3.5 is in complete analogy to the following standard result for Gaussian processes (as illustrated in the sketches in the introduction):

a) A function $C: T \times T \rightarrow \mathbb{R}$ is a covariance function if and only if it is positive definite.

b) If $C: T \times T \rightarrow \mathbb{R}$ is positive definite, we may choose a (zero mean) Gaussian process which realizes $C$ as its own covariance function.

Both statements are intrinsically tied together. When proving them by means of Kolmogorov's extension theorem, one proceeds in the same manner as we did for Theorem 2.3.5. The necessity of positive definiteness of covariance functions is easily derived even for the bigger class of square-integrable processes, whilst sufficiency can be established by showing that Gaussian processes can realize any positive definite function as covariance function. 
Remark 2.3.6. In order to incorporate stationarity w.r.t. some group $G$ acting (e.g. from the left) on $T$ (for example $\mathbb{R}^{d}$ acting on $\mathbb{R}^{d}$ by translation), we just have to add the following condition (iv) $\theta(g A)=\theta(A)$ for all $A \in \mathcal{F}(T) \backslash\{\emptyset\}$ and for all $g \in G$. Then the process $X^{*}$ will be stationary w.r.t. this group action.

Remark 2.3.7. Instead of requiring the max-stable processes in Theorem 2.3.5 to have standard Fréchet marginals everywhere, we can admit a different scale at different locations, i.e. $\mathbb{P}\left(X_{t} \leq x\right)=\exp \left(-s_{t} / x\right)$ for a positive scaling parameter $s_{t}$ for $t \in T$. In this case Theorem 2.3 .5 holds true without condition (iii) and the word "simple". To make sense of the ECF as in (2.1) in this case, either use a reference point $t \in T$ or set $\log \mathbb{P}\left(X_{t} \leq x\right)=-1 / x$ in the denominator. Beware of that the ECF $\theta$ cannot be interpreted as the number of independent variables anymore in this case.

Remark 2.3.8. In [85] the last issue of the proof is derived for finite sets $T$ by a Moebius inversion. The relation to the proof therein becomes more transparent if we compute $\theta^{*}(A)$ for $A \subset M$ from the coefficients $\left\{\tau_{L}^{M}\right\}_{\emptyset \neq L \subset M}$ for arbitrary $M \supset A$ instead of $M=A$ :

$$
\theta^{*}(A) \stackrel{2.1,2.7]}{=} \sum_{\emptyset \neq K \subset A} \tau_{K}^{A} \stackrel{2.9}{=} \sum_{\emptyset \neq K \subset A} \sum_{J \subset M \backslash A} \tau_{K \cup J}^{M}=\sum_{L \subset M: L \cap A \neq \emptyset} \tau_{L}^{M} .
$$

\subsection{Consequences of negative definiteness}

Here, we collect some immediate consequences of the above Theorem 2.3.5. Therefore, note that the first part of Theorem 2.3.5 can also be expressed as (cf. (2.2))

$$
\Theta(T)=\left\{\theta: \mathcal{F}(T) \rightarrow \mathbb{R}: \begin{array}{l}
\theta \text { is negative definite (completely alternating) } \\
\text { on } \mathcal{F}(T), \theta(\emptyset)=0, \theta(\{t\})=1 \text { for } t \in T .
\end{array}\right\}
$$

\subsubsection{Convexity and compactness}

Corollary 2.4.1. The set of ECFs $\Theta(T)$ is convex.

Proof. This can be seen readily from (2.16) since all involved properties are compatible with convex combinations. As a constructive argument use the fact that the ECF of the max-combination $\alpha X \vee(1-\alpha) Y$ of two independent simple max-stable processes $X$ and $Y$ on $T$ is the convex combination of their ECFs for $\alpha \in(0,1)$.

Corollary 2.4.2. The set of ECFs $\Theta(T)$ is compact w.r.t. the topology of pointwise convergence. 
Proof. The topology of pointwise convergence on $\mathbb{R}^{\mathcal{F}(T)}$ is the product topology. Since $\theta(\emptyset)=0$ and $\theta(A) \in[1,|A|]$ for $\theta \in \Theta(T)$ and $A \in \mathcal{F}(T) \backslash\{\emptyset\}$, the set $\Theta(T)$ is a subset of the product space

$$
\Theta(T) \subset\{0\} \times \prod_{A \in \mathcal{F}(T) \backslash\{\emptyset\}}[1,|A|]
$$

which is compact by Tychonoff's theorem. Moreover, since elements of $\Theta(T)$ are completely characterized by finite-dimensional equalities and inequalities involving $\leq$ only (stemming from (2.16), the set $\Theta(T)$ is closed. Hence $\Theta(T)$ is compact.

Remark 2.4.3. Note that even though we say "the topology of pointwise convergence", the "points" meant here are indeed elements of $\mathcal{F}(T)$, i.e. finite subsets of $T$. In particular it follows from the compactness of $\Theta(T)$ that $\Theta(T)$ is sequentially closed. That means if $\left(\theta_{n}\right)_{n \in \mathbb{N}}$ is a sequence of ECFs such that $\theta_{n}(A)$ converges to some value $f(A)$ for each $A \in \mathcal{F}(T)$, then $f$ is an ECF.

\subsubsection{Spectral representations}

Another consequence of Theorem 2.3.5 is that ECFs allow for an integral representation as a mixture of functions $A \mapsto \mathbb{1}_{A \cap Q \neq \emptyset}$, where $Q$ is from the power set of $T$. To be more precise, let us denote the power set of $T$ by $\mathcal{P}(T)$ and consider the topology on $\mathcal{P}(T)$ that is generated by the maps $Q \mapsto \mathbb{1}_{A \cap Q \neq \emptyset}$ for $A \in \mathcal{F}(T)$ or equivalently (since $\mathcal{F}(T)$ is generated by the singletons $\{\{t\}\}_{t \in T}$ ) the topology on $\mathcal{P}(T)$ that is generated by the maps $Q \mapsto \mathbb{1}_{t \in Q}$ for $t \in T$. Identifying $\mathcal{P}(T)$ with $\{0,1\}^{T}$, this space is also known as Cantor cube. As in [5, Definition 2.1.1.] a measure $\mu$ on the Borel $\sigma$-algebra of $\mathcal{P}(T)$ w.r.t. this topology will be called Radon measure if $\mu$ is finite on compact sets and $\mu$ is inner regular.

Corollary 2.4.4. Let $\theta \in \Theta(T)$ be an ECF. Then $\theta$ uniquely determines a positive Radon measure $\mu$ on $\mathcal{P}(T) \backslash\{\emptyset\}$ such that

$$
\theta(A)=\mu(\{Q \in \mathcal{P}(T) \backslash\{\emptyset\}: A \cap Q \neq \emptyset\})=\int_{\mathcal{P}(T) \backslash\{\emptyset\}} \mathbb{1}_{A \cap Q \neq \emptyset} \mu(\mathrm{d} Q),
$$

where $\theta(\{t\})=1$ for $t \in T$. Moreover, the function $\theta$ is bounded if and only if $\mu(\mathcal{P}(T) \backslash\{\emptyset\})<\infty$. 
Proof. Since $\theta$ is negative definite (Theorem 2.3.5) and $\mathcal{F}(T)$ is idempotent, we may apply Theorem A.1.10, which says that $\theta$ uniquely determines a positive Radon measure $\widetilde{\mu}$ on $\widehat{\mathcal{F}(T)} \backslash\{1\}$, where $\widehat{\mathcal{F}(T)}$ denotes the dual semigroup of $\mathcal{F}(T)$ (cf. Definition A.1.4, such that $\theta(A)=\widetilde{\mu}(\{\rho \in \widehat{\mathcal{F}(T)} \backslash\{1\} \mid \rho(A)=0\})$. The function $\theta$ is bounded if and only if $\widetilde{\mu}(\widehat{\mathcal{F}(T)} \backslash\{1\})<\infty$.

Now, it can be easily seen (using $\left.\rho(A)=\prod_{t \in A} \rho(\{t\})=\bigwedge_{t \in A} \rho(\{t\})\right)$ that semicharacters on $\mathcal{F}(T)$ are in a 1-1 correspondence with subsets of $T$ via

$$
\begin{aligned}
& \widehat{\mathcal{F}(T)} \stackrel{1-1}{\longleftrightarrow} \mathcal{P}(T) \\
& \rho \longmapsto\{t \in T: \rho(\{t\})=0\} \\
& \mathbb{1}_{(\cdot) \cap Q=\emptyset} \longleftrightarrow Q
\end{aligned}
$$

Here the constant function 1 corresponds to the empty set. Moreover, the topology considered on $\widehat{\mathcal{F}(T)}$ is the topology of pointwise convergence. Transported to $\mathcal{P}(T)$ this is the topology generated by the maps $Q \mapsto \mathbb{1}_{A \cap Q \neq \emptyset}$ for $A \in \mathcal{F}(T)$. Let $\mu$ denote the Radon measure $\tilde{\mu}$ transported to $\mathcal{P}(T) \backslash\{\emptyset\}$. Then the corollary follows.

Remark 2.4.5. In case $T=M$ is finite, we have that $\mathcal{P}(M)=\mathcal{F}(M)$ carries the discrete topology and

$$
\theta(A)=\mu(\{Q \in \mathcal{F}(M) \backslash\{\emptyset\}: A \cap Q \neq \emptyset\})=\sum_{Q \in \mathcal{F}(M) \backslash\{\emptyset\}} \mu(\{Q\}) \mathbb{1}_{A \cap Q \neq \emptyset}
$$

A comparison with 2.15 reveals that $\mu(\{Q\})=\tau_{Q}^{M}$. In this sense, the coefficients $\tau_{Q}^{M}$ of the max-linear model 2.7 can be interpreted as finite-dimensional "Fourier coefficients" of the negative definite function $\theta$. Moreover, denoting $\mathscr{F}(M)$ the set of sub-semigroups of $\mathcal{F}(M)$ that are hereditary on the left (see before Theorem A.1.10, we have the following (topological) isomorphisms of idempotent abelian (discrete) semigroups

$$
(\widehat{\mathcal{F}(M)}, \cdot, 1) \cong(\mathscr{F}(M), \cap, \mathcal{F}(M)) \cong(\mathcal{F}(M), \cap, M) \cong(\mathcal{F}(M), \cup, \emptyset),
$$

where the respective maps are given by $\rho \mapsto \rho^{-1}(\{1\}), I \mapsto \bigcup_{A \in I} A, C \mapsto C^{c}$ (forward) and $Q \mapsto Q^{c}, C \mapsto \mathcal{F}(C), I \mapsto \mathbb{1}_{I}$ (backward). In particular the semigroup $(\mathcal{F}(M), \cup, \emptyset)$ is isomorphic to its dual semigroup $(\widehat{\mathcal{F}(M)}, \cdot, 1)$ in this case.

The integral representation of the ECF $\theta$ also yields a spectral representation $(\Omega, \mathcal{A}, \nu, V)$ for the corresponding process $X^{*}$ (cf. Definition (1.1.1)). 
Theorem 2.4.6. Let $X^{*}=\left\{X_{t}^{*}\right\}_{t \in T}$ be the simple max-stable process from Theorem 2.3.5 b) with ECF $\theta$. Then $X^{*}$ has the following spectral representation $(\Omega, \mathcal{A}, \nu, V)$ :

- $(\Omega, \mathcal{A}, \nu)$ is the measure space $(\mathcal{P}(T), \mathcal{B}(\mathcal{P}(T)), \mu)$ from Corollary 2.4.4.

- $V_{t}(Q)=\mathbb{1}_{t \in Q}$

Proof. We need to check that the f.d.d. of $X^{*}$ satisfy (1.6). The f.d.d. of $X^{*}$ are given by (2.8)

$$
-\log \mathbb{P}\left(X_{t}^{*} \leq x_{t}, t \in M\right)=\sum_{\emptyset \neq L \subset M} \tau_{L}^{M} \bigvee_{t \in L} \frac{1}{x_{t}}
$$

where the coefficients $\tau_{L}^{M}$ can be computed from the ECF $\theta$ as in Theorem 2.3.5 b and $\theta$ satisfies the integral representation from Corollary 2.4.4, i.e.

$$
\tau_{L}^{M}=\sum_{I \subset L}(-1)^{|I|+1} \theta((M \backslash L) \cup I)=\sum_{I \subset L}(-1)^{|I|+1} \int_{\mathcal{P}(T) \backslash\{\emptyset\}} \mathbb{1}_{((M \backslash L) \cup I) \cap Q \neq \emptyset} \mu(\mathrm{d} Q) .
$$

Using the identity

$$
\begin{aligned}
& \sum_{I \subset L}(-1)^{|I|+1} \mathbb{1}_{((M \backslash L) \cup I) \cap Q \neq \emptyset} \\
& =\sum_{I \subset L}(-1)^{|I|+1}\left(\mathbb{1}_{(M \backslash L) \cap Q \neq \emptyset}+\mathbb{1}_{I \cap Q \neq \emptyset}-\mathbb{1}_{(M \backslash L) \cap Q \neq \emptyset} \mathbb{1}_{I \cap Q \neq \emptyset}\right) \\
& =0 \cdot \mathbb{1}_{(M \backslash L) \cap Q \neq \emptyset}+\left(1-\mathbb{1}_{(M \backslash L) \cap Q \neq \emptyset}\right) \sum_{I \subset L}(-1)^{|I|+1} \mathbb{1}_{I \cap Q \neq \emptyset} \\
& =\mathbb{1}_{(M \backslash L) \cap Q=\emptyset} \mathbb{1}_{L \subset Q}=\mathbb{1}_{L=M \cap Q},
\end{aligned}
$$

we obtain that

$$
\tau_{L}^{M}=\int_{\mathcal{P}(T) \backslash\{\emptyset\}} \mathbb{1}_{L=M \cap Q} \mu(\mathrm{d} Q) .
$$

It follows that the f.d.d. of $X^{*}$ satisfy

$$
\begin{aligned}
-\log \mathbb{P}\left(X_{t}^{*} \leq x_{t}, t \in M\right) & =\int_{\mathcal{P}(T) \backslash\{\emptyset\}} \sum_{\emptyset \neq L \subset M} \mathbb{1}_{L=M \cap Q} \bigvee_{t \in L} \frac{1}{x_{t}} \mu(\mathrm{d} Q) \\
& =\int_{\mathcal{P}(T) \backslash\{\emptyset\}} \bigvee_{t \in M} \frac{\mathbb{1}_{t \in Q}}{x_{t}} \mu(\mathrm{d} Q)=\int_{\Omega}\left(\bigvee_{t \in M} \frac{V_{t}(\omega)}{x_{t}}\right) \nu(\mathrm{d} \omega)
\end{aligned}
$$

as desired. This finishes the proof. 


\subsubsection{Triangle inequalities and the operation of Bernstein functions}

In [11, Proposition 4] it is shown that an ECF $\theta$ on $\mathcal{F}(T)$ satisfies the following bivariate inequalities for $r, s, t \in T$ :

$$
\begin{aligned}
\theta(\{s, t\}) & \leq \theta(\{s, r\}) \theta(\{r, t\}), & & \\
\theta(\{s, t\})^{\alpha} & \leq \theta(\{s, r\})^{\alpha}+\theta(\{r, t\})^{\alpha}-1, & & 0<\alpha \leq 1, \\
\theta(\{s, t\})^{\alpha} & \geq \theta(\{s, r\})^{\alpha}+\theta(\{r, t\})^{\alpha}-1, & & \alpha \leq 0 .
\end{aligned}
$$

These inequalities have in common, that they are in fact triangle inequalities of the form

$$
g \circ \eta(\{s, t\}) \leq g \circ \eta(\{s, r\})+g \circ \eta(\{r, t\}),
$$

if we rewrite them in terms of $\eta:=\theta-1$ and

$$
\begin{aligned}
& g(x)=\log (1+x), \\
& g(x)=(1+x)^{\alpha}-1, \quad 0<\alpha \leq 1, \\
& g(x)=1-(1+x)^{\alpha}, \quad \alpha \leq 0 .
\end{aligned}
$$

These functions $g$ have in common that they are in fact Bernstein functions.

Theorem/Definition 2.4.7 (Bernstein function).

A function $g:[0, \infty) \rightarrow[0, \infty)$ is called a Bernstein function if one of the following equivalent conditions is satisfied (cf. [5, 4.4.3 and p. 141]):

(i) The function $g$ is of the form

$$
g(r)=c+b r+\int_{0}^{\infty}\left(1-e^{-\lambda r}\right) \nu(\mathrm{d} \lambda)
$$

where $c, b \geq 0$ and $\nu$ is a positive Radon measure on $(0, \infty)$ with $\int_{0}^{\infty} \frac{\lambda}{1+\lambda} \nu(\mathrm{d} \lambda)<$ $\infty$ (or equivalently $\int_{0}^{\infty} \min (\lambda, 1) \nu(\mathrm{d} \lambda)<\infty$ ).

(ii) The function $g$ is continuous and $g \in C^{\infty}((0, \infty))$ with $g \geq 0$ and $(-1)^{n} g^{(n+1)} \geq$ 0 for all $n \geq 0$. (Here, $g^{(n)}$ denotes the $n$-th derivative of $g$.)

(iii) The function $g$ is continuous, $g \geq 0$ and $g$ is negative definite as a function on the semigroup $([0, \infty),+, 0)$ (cf. Definition A.1.1).

(iv) The function $\varphi \circ g$ is completely monotone on $[0, \infty)$ for all completely monotone functions $\varphi$, where "completely monotone" is to be understood in the sense of Definition A.2.3. 
For a comprehensive treatise on Bernstein functions including a table of examples see [82. Bernstein functions play already an important role in the construction of advanced Gaussian processes by generating novel covariance functions from given ones, cf. [102] and [78]. Here we see that they are equally useful for generating new ECFs from given ECFs and correspondingly new max-stable processes from given ones.

Corollary 2.4.8. Let $T$ be a set and $\theta \in \Theta(T)$ an ECF. Let $g$ be a Bernstein function which is not constant. Then the function on $\mathcal{F}(T)$

$$
A \mapsto \frac{g(\theta(A))-g(0)}{g(1)-g(0)}
$$

is again an ECF in $\Theta(T)$.

Proof. The result is immediate from Theorem 2.3.5, since Bernstein functions operate on negative definite kernels (cf. [5, 3.2.9 and 4.4.3]).

For instance, if $\theta$ is an ECF, then also $\log (1+\theta) / \log (2)$ or $\left((\theta+a)^{q}-a^{q}\right) /((1+$ $\left.a)^{q}-a^{q}\right)$ are ECFs for $0<q<1$ and $a \geq 0$. Finally, the result of [11, Proposition 4] can be generalized to the following extent as a corollary to Theorem 2.3.5.

Corollary 2.4.9. Let $\theta \in \Theta(T)$ be an ECF. Set $\eta:=\theta-1$ and let $g$ be a Bernstein function. Then we have for $A, B, C \in \mathcal{F}(T) \backslash\{\emptyset\}$ that

$$
g \circ \eta(A \cup B) \leq g \circ \eta(C)+g \circ \eta(A \cup B) \leq g \circ \eta(A \cup C)+g \circ \eta(C \cup B) .
$$

Proof. Since $\theta$ is an ECF, it is negative definite (cf. Theorem 2.3.5). Subtracting 1 does not change this property. Notice further that $\theta$ takes values in $\{0\} \cup[1, \infty)$, where the value 0 is only attained for the empty set $\emptyset$ (the neutral element of $\mathcal{F}(T)$ ). Thus, $\eta=\theta-1: \mathcal{F}(T) \backslash\{\emptyset\} \rightarrow \mathbb{R}$ is negative definite and takes values only in $[0, \infty)$. Applying a Bernstein function $g$ does not change this property (cf. [5, 3.2.9. and 4.4.3.]). By [5, 8.2.7.], this also means that $f:=g \circ \eta: \mathcal{F}(T) \backslash\{\emptyset\} \rightarrow \mathbb{R}$ is completely alternating on $\mathcal{F}(T) \backslash\{\emptyset\}$. Since we have also $f \geq 0$ on $\mathcal{F}(T) \backslash\{\emptyset\}$, we may derive for $A, B, C \in \mathcal{F}(T) \backslash\{\emptyset\}$

$$
\begin{aligned}
& f(C)+f(A \cup B)-f(A \cup C)-f(C \cup B) \\
& =(f(C)-f(A \cup C)-f(C \cup B)+f(A \cup B \cup C))+(f(A \cup B)-f(A \cup B \cup C)) \\
& =\Delta_{A} \Delta_{B} f(C)+\Delta_{C} f(A \cup B) \leq 0
\end{aligned}
$$

as desired. This finishes the proof. 


\subsection{Continuity}

In this section we require $T$ to be a metric space. We need to define the notion of continuity that we will use in connection with ECFs $\theta: \mathcal{F}(T) \rightarrow[0, \infty)$. Therefore let $f: \mathcal{F}(T) \rightarrow \mathbb{R}$ be a function on the finite subsets of $T$. Then $f$ induces a familiy of functions $\left\{f^{(m)}\right\}_{m \geq 0}$ where $f^{(m)}: T^{m} \rightarrow \mathbb{R}$ is given by

$$
f^{(m)}\left(t_{1}, \ldots, t_{m}\right)=f\left(\left\{t_{1}, \ldots, t_{m}\right\}\right)
$$

Definition 2.5.1. Let $f: \mathcal{F}(T) \rightarrow \mathbb{R}$ be a function on the finite subsets of a metric space $T$. We say that $f$ is continuous if all induced functions $f^{(m)}: T^{m} \rightarrow \mathbb{R}$ are continuous for all $m \geq 0$, where $T^{m}$ is endowed with the product topology.

Lemma 2.5.2. Let $X=\left\{X_{t}\right\}_{t \in T}$ be a simple max-stable process with ECF $\theta$. Then the following implication holds:

$$
X \text { is stochastically continuous. } \Longrightarrow \theta \text { is continuous. }
$$

Proof. Stochastic continuity of $X$ means that for any $\varepsilon>0$, for any $t \in T$ and sequence $t^{(n)} \rightarrow t$ we have $\mathbb{P}\left(\left|X_{t^{(n)}}-X_{t}\right|>\varepsilon\right) \rightarrow 0$. From this, we can easily derive that for any $\varepsilon>0$, any $m \in \mathbb{N}$, any $\left(t_{1}, \ldots, t_{m}\right) \in T^{m}$ and a sequence $\left(t_{1}^{(n)}, \ldots, t_{m}^{(n)}\right) \rightarrow\left(t_{1}, \ldots, t_{m}\right)$, also $\mathbb{P}\left(\left\|\left(X_{t_{i}^{(n)}}-X_{t_{i}}\right)_{i=1}^{m}\right\|>\varepsilon\right) \rightarrow 0$ for any reference norm $\|\cdot\|$ on $\mathbb{R}^{m}$. The latter implies the corresponding convergence in distribution: $F_{\left(t_{1}^{(n)}, \ldots, t_{m}^{(n)}\right)} \rightarrow F_{\left(t_{1}, \ldots, t_{m}\right)}$. Since $\log F_{\left(t_{1}, \ldots, t_{m}\right)}:[0, \infty)^{m} \rightarrow \mathbb{R}$ is monotone and homogeneous, we have that for $x>0$ the point $(x, \ldots, x) \in(0, \infty)^{m}$ is a continuity point of $F_{\left(t_{1}, \ldots, t_{m}\right)}$ (cf. [79, p. 277]). Thus, the induced function $\theta^{(m)}$ on $T^{m}$ is continuous, since $\theta^{(m)}\left(t_{1}, \ldots, t_{m}\right)=-x \log F_{\left(t_{1}, \ldots, t_{m}\right)}(x, \ldots, x)$. Hence $\theta$ is continuous.

Secondly, we prove the following upper bound that shows that stochastic continuity of the process $X^{*}$ is indeed controlled by the bivariate extremal coefficients:

Lemma 2.5.3. Let $X^{*}=\left\{X_{t}^{*}\right\}_{t \in T}$ be the simple max-stable process from Theorem 2.3.5 bith ECF $\theta$. Set $\eta:=\theta-1$. Then we have for any $\varepsilon>0$

$$
\mathbb{P}\left(\left|X_{s}^{*}-X_{t}^{*}\right|>\varepsilon\right) \leq 2\left(1-\exp \left(-\frac{\eta(\{s, t\})}{\varepsilon}\right)\right) \leq \frac{2}{\varepsilon} \eta(\{s, t\}) .
$$


Proof. Let $\varepsilon>0$. We will prove the statement for $2 \varepsilon$ instead of $\varepsilon$. Therefore, consider the following disjoint events on a corresponding probability space $(\Omega, \mathcal{A}, \mathbb{P})$ for $k=0,1,2, \ldots$

$$
A_{k}:=\left\{\omega \in \Omega:\left(X_{s}^{*}(\omega), X_{t}^{*}(\omega)\right) \in(k \varepsilon,(k+2) \varepsilon]^{2} \backslash((k+1) \varepsilon,(k+2) \varepsilon]^{2}\right\} .
$$

The disjoint union $\bigcup_{k=0}^{\infty} A_{k}$ is a subset of $\left\{\omega \in \Omega:\left|X_{s}^{*}(\omega)-X_{t}^{*}(\omega)\right| \leq 2 \varepsilon\right\}$ and so

$$
\mathbb{P}\left(\left|X_{s}^{*}-X_{t}^{*}\right| \leq 2 \varepsilon\right) \geq \mathbb{P}\left(\bigcup_{k=0}^{\infty} A_{k}\right)=\sum_{k=0}^{\infty} \mathbb{P}\left(A_{k}\right)=\lim _{n \rightarrow \infty} \sum_{k=0}^{n} \mathbb{P}\left(A_{k}\right)
$$

From 2.8 and Theorem 2.3.5 we see that the bivariate distribution of the process $X^{*}$ is given by

$$
-\log \mathbb{P}\left(X_{s}^{*} \leq x, X_{t}^{*} \leq y\right)=\frac{\eta(\{s, t\})}{x \vee y}+\frac{1}{x \wedge y}
$$

For further calculations we abbreviate for $p, q \in \mathbb{N} \cup\{0\}$

$$
B(p, q):=\mathbb{P}\left(X_{s}^{*} \leq p \cdot \varepsilon, X_{t}^{*} \leq q \cdot \varepsilon\right) .
$$

Note that $B(p, q)=B(q, p)$ and $B(p, 0)=0$. With this notation we rearrange

$$
\sum_{k=0}^{n} \mathbb{P}\left(A_{k}\right)=-B(n+1, n+1)+2 \sum_{k=0}^{n}[B(k+2, k+1)-B(k+2, k)] .
$$

For the second summand we have (cf. 2.17)

$$
\begin{aligned}
& \sum_{k=0}^{n}[B(k+2, k+1)-B(k+2, k)] \\
& \stackrel{2.17}{-} \sum_{k=0}^{n}\left[\exp \left(-\frac{1}{\varepsilon}\left[\frac{\eta(\{s, t\})}{k+2}+\frac{1}{k+1}\right]\right)-\exp \left(-\frac{1}{\varepsilon}\left[\frac{\eta(\{s, t\})}{k+2}+\frac{1}{k}\right]\right)\right] \\
& =\sum_{k=0}^{n} \exp \left(-\frac{1}{\varepsilon}\left[\frac{\eta(\{s, t\})}{k+2}\right]\right)\left[\exp \left(-\frac{1}{(k+1) \varepsilon}\right)-\exp \left(-\frac{1}{k \varepsilon}\right)\right] \\
& \geq \sum_{k=0}^{n} \exp \left(-\frac{\eta(\{s, t\})}{2 \varepsilon}\right)\left[\exp \left(-\frac{1}{(k+1) \varepsilon}\right)-\exp \left(-\frac{1}{k \varepsilon}\right)\right] \\
& =\exp \left(-\frac{\eta(\{s, t\})}{2 \varepsilon}\right) \exp \left(-\frac{1}{(n+1) \varepsilon}\right) .
\end{aligned}
$$


Finally,

$$
\begin{aligned}
& \mathbb{P}\left(\left|X_{s}^{*}-X_{t}^{*}\right|>2 \varepsilon\right)=1-\mathbb{P}\left(\left|X_{s}^{*}-X_{t}^{*}\right| \leq 2 \varepsilon\right) \leq 1-\lim _{n \rightarrow \infty} \sum_{k=0}^{n} \mathbb{P}\left(A_{k}\right) \\
& =1+\lim _{n \rightarrow \infty} B(n+1, n+1)-2 \lim _{n \rightarrow \infty} \sum_{k=0}^{n}[B(k+2, k+1)-B(k+2, k)] \\
& \quad \leq 1+\lim _{n \rightarrow \infty} \exp \left(-\frac{\eta(\{s, t\})+1}{(n+1) \varepsilon}\right)-2 \lim _{n \rightarrow \infty}\left(\exp \left(-\frac{\eta(\{s, t\})}{2 \varepsilon}\right) \exp \left(-\frac{1}{(n+1) \varepsilon}\right)\right) \\
& =2-2 \exp \left(-\frac{\eta(\{s, t\})}{2 \varepsilon}\right) \leq \frac{2}{2 \varepsilon} \eta(\{s, t\}) .
\end{aligned}
$$

This finishes the proof.

Theorem 2.5.4. Let $X^{*}=\left\{X_{t}^{*}\right\}_{t \in T}$ be the simple max-stable process from Theorem 2.3.5 b) with ECF $\theta$. Then the following statements are equivalent:

(i) $X^{*}$ is stochastically continuous.

(ii) $\theta$ is continuous.

(iii) The bivariate map $(s, t) \mapsto \theta(\{s, t\})$ is continuous.

(iv) The bivariate map $(s, t) \mapsto \theta(\{s, t\})$ is continuous on the diagonal.

Proof. The implication $(i) \Rightarrow(i i)$ follows from Lemma 2.5.2 Clearly, continuity of $\theta$ implies continuity of the induced function $\theta^{(2)}(s, t):=\theta(\{s, t\})$, which implies continuity of $\theta^{(2)}$ on the diagonal. This shows the implications $(i i) \Rightarrow(i i i)$ and $($ iii $) \Rightarrow(i v)$. Finally, the implication $(i v) \Rightarrow(i)$ follows from Lemma 2.5.3. since $\eta(\{t, t\})=\theta(\{t\})-1=0$.

\subsection{Dependency sets}

In this section we show that the max-stable process $X^{*}$ is exceptional among all maxstable processes sharing the same ECF $\theta$ as $X^{*}$ in the sense that its dependency set $\mathcal{K}^{*}$ (to be introduced below) is maximal w.r.t. inclusion.

Therefore, recall that for a finite non-empty subset $M \subset T$ the dependency set $\mathcal{K}_{M}$ of $\left\{X_{t}\right\}_{t \in M}$ is the largest compact convex set $\mathcal{K}_{M} \subset[0, \infty)^{M}$ satisfying (cf. (1.3))

$$
\ell_{M}(x)=\sup \left\{\langle x, y\rangle: y \in \mathcal{K}_{M}\right\} \quad \forall x \in[0, \infty)^{M} .
$$


The closed convex set $\mathcal{K}_{M}$ may also be described as the following intersection of half spaces (cf. [87, Section 1.7.]):

$$
\mathcal{K}_{M}=\bigcap_{x \in S_{M}}\left\{y \in[0, \infty)^{M}:\langle x, y\rangle \leq \ell_{M}(x)\right\}
$$

Example 2.6.1 ([6], Example 1 and Proposition 2). The simplest examples for dependency sets $\mathcal{K}_{M}$ are the unit cube $[0,1]^{M}$ corresponding to a collection of independent random variables $\left\{X_{t}\right\}_{t \in M}$ and the cross-polytope $D^{M}:=\left\{x \in[0, \infty)^{M}\right.$ : $\left.\sum_{t \in M} x_{t} \leq 1\right\}$ corresponding to identical random variables $\left\{X_{t}\right\}_{t \in M}$. Any dependency set $\mathcal{K}_{M}$ of a simple max-stable distribution satisfies

$$
D^{M} \subset \mathcal{K}_{M} \subset[0,1]^{M}
$$

Example 2.6.2 (Brown-Resnick process, Hüsler-Reiss distribution). The f.d.d. of a Brown-Resnick process (cf. Example 2.1.3) are the multivariate Hüsler-Reiss distributions (cf. [44). In the bivariate case, when $M=\{1,2\}$ consists of two points only, the distribution function of a Hüsler-Reiss distributed random vector $\left(X_{1}, X_{2}\right)$, standardized to unit Fréchet marginals, is

$$
-\log \mathbb{P}_{\gamma}\left(X_{1} \leq x_{1}, X_{2} \leq x_{2}\right)=\frac{1}{x_{1}} \Phi\left(\frac{\sqrt{\gamma}}{2}+\frac{\log \left(x_{2} / x_{1}\right)}{\sqrt{\gamma}}\right)+\frac{1}{x_{2}} \Phi\left(\frac{\sqrt{\gamma}}{2}+\frac{\log \left(x_{1} / x_{2}\right)}{\sqrt{\gamma}}\right)
$$

for $x_{1}, x_{2} \geq 0$. Here $\Phi$ denotes the distribution function of the standard normal distribution and the parameter $\gamma$ is the value of the variogram between the two points (cf. Example 2.1.3). Figure 2.2 illustrates, how the corresponding dependency sets range between full dependence $(\gamma=0)$ and independence $(\gamma=\infty)$.

In order to define a single dependency set for a simple max-stable process comprising all multivariate dependency sets, we write

$$
\operatorname{pr}_{M}:[0, \infty)^{T} \rightarrow[0, \infty)^{M} \quad\left(x_{t}\right)_{t \in T} \mapsto\left(x_{t}\right)_{t \in M}
$$

for the natural projection.

Definition 2.6.3. Let $X$ be a simple max-stable process $X=\left\{X_{t}\right\}_{t \in T}$ and denote for finite $M \in \mathcal{F}(T) \backslash\{\emptyset\}$ the multivariate dependency set of the random vectors $\left\{X_{t}\right\}_{t \in M}$ by $\mathcal{K}_{M}$. Then we define the dependency set $\mathcal{K} \subset[0, \infty)^{T}$ of $X$ as

$$
\mathcal{K}:=\bigcap_{M \in \mathcal{F}(T) \backslash\{\emptyset\}} \operatorname{pr}_{M}^{-1}\left(\mathcal{K}_{M}\right)
$$




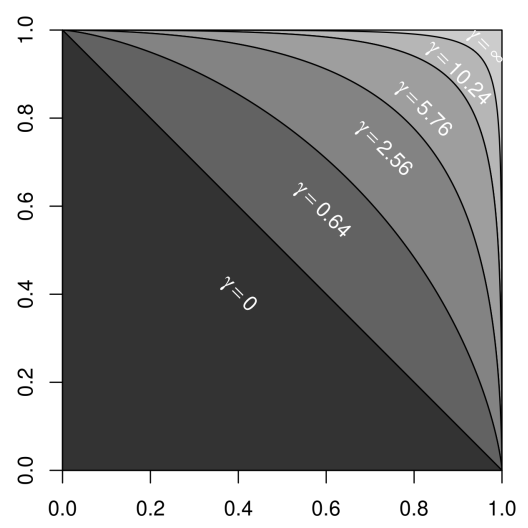

Figure 2.2.: Nested dependency sets $\mathcal{K}_{M}^{(\gamma)}$ of the bivariate Brown-Resnick (resp. HüslerReiss) distribution where $M=\{1,2\}$ (cf. Example 2.6.2). The dependency sets grow as the parameter $\gamma$ increases. They range between full dependence $(\gamma=0)$ and independence $(\gamma=\infty)$.

Analogously to 1.3 , the dependency set $\mathcal{K}$ may be characterized as follows.

Lemma 2.6.4. The dependency set $\mathcal{K}$ of a simple max-stable process $X=\left\{X_{t}\right\}_{t \in T}$ is the largest compact convex set $\mathcal{K} \subset[0, \infty)^{T}$ satisfying

$$
\ell_{M}(x)=\sup \left\{\sum_{t \in M} x_{t} y_{t}: y \in \mathcal{K}\right\} \quad \forall x \in[0, \infty)^{M} \forall \emptyset \neq M \in \mathcal{F}(T),
$$

where $\ell_{M}$ is the stable tail dependence function of $\left\{X_{t}\right\}_{t \in M}$.

Proof. Convexity of $\mathcal{K}$ follows from the convexity of each $\mathcal{K}_{M}$ and from the linearity of the projections $\operatorname{pr}_{M}$ for $M \in \mathcal{F}(T) \backslash\{\emptyset\}$. Since $\mathcal{K}_{\{t\}}=[0,1]$ is the unit interval for each $t \in T$, the set $\mathcal{K}$ is contained in the compact space $[0,1]^{T}$. Moreover, $\mathcal{K}$ is closed as the intersection of closed sets, hence $\mathcal{K}$ is compact.

Next, we prove that $\mathcal{K}_{M}=\operatorname{pr}_{M}(\mathcal{K})$. By definition of $\mathcal{K}$ it is clear that $\operatorname{pr}_{M}(\mathcal{K}) \subset$ $\mathcal{K}_{M}$ for $M \in \mathcal{F}(T) \backslash\{\emptyset\}$. To prove the reverse inclusion, let $y_{M}$ be an element of $\mathcal{K}_{M}$ and set $V\left(y_{M}\right):=\operatorname{pr}_{M}^{-1}\left(\left\{y_{M}\right\}\right) \cap \mathcal{K}=\operatorname{pr}_{M}^{-1}\left(\left\{y_{M}\right\}\right) \cap \mathcal{K} \cap[0,1]^{T}$. We need to show that $V\left(y_{M}\right) \neq \emptyset$. Denoting $V\left(y_{M}, A\right):=\operatorname{pr}_{M}^{-1}\left(\left\{y_{M}\right\}\right) \cap \operatorname{pr}_{A}^{-1}\left(\mathcal{K}_{A}\right) \cap[0,1]^{T}$, we see that

$$
V\left(y_{M}\right)=\bigcap_{A \in \mathcal{F}(T) \backslash\{\emptyset\}} V\left(y_{M}, A\right) .
$$

Note that each $V\left(y_{M}, A\right)$ is a closed subset of the compact Hausdorff space $[0,1]^{T}$. 
Therefore, it suffices to verify the finite intersection property for the system of sets

$$
\left\{V\left(y_{M}, A\right)\right\}_{A \in \mathcal{F}(T) \backslash\{\emptyset\}}
$$

in order to show $V\left(y_{M}\right) \neq \emptyset$ (cf. e.g. [3, Theorem 2.31]). But this follows from the consistency of the finite-dimensional dependency sets $\left\{\mathcal{K}_{A}\right\}_{A \in \mathcal{F}(T) \backslash\{\emptyset\}}$ as follows: As [66, Section 7 (Projection)] essentially says, we have that if $A$ and $B$ are non-empty finite subsets of $T$ with $A \subset B$, then $\mathcal{K}_{A}$ is the projection of $\mathcal{K}_{B}$ onto the respective coordinate space. In particular $\operatorname{pr}_{B}^{-1}\left(\mathcal{K}_{B}\right) \subset \operatorname{pr}_{A}^{-1}\left(\mathcal{K}_{A}\right)$ and $\operatorname{pr}_{A}^{-1}\left(\left\{y_{A}\right\}\right) \cap \operatorname{pr}_{B}^{-1}\left(\mathcal{K}_{B}\right) \cap$ $[0,1]^{T} \neq \emptyset$ for $y_{A} \in \mathcal{K}_{A}$. Now, let $A_{1}, \ldots, A_{k}$ be non-empty finite subsets of $T$. Then

$$
\begin{aligned}
\emptyset & \neq \operatorname{pr}_{M}^{-1}\left(\left\{y_{M}\right\}\right) \cap \operatorname{pr}_{M \cup \bigcup_{i=1}^{k} A_{i}}^{-1}\left(\mathcal{K}_{M \cup \bigcup_{i=1}^{k} A_{i}}\right) \cap[0,1]^{T} \\
& \subset \operatorname{pr}_{M}^{-1}\left(\left\{y_{M}\right\}\right) \cap \bigcap_{i=1}^{k} \operatorname{pr}_{A_{i}}^{-1}\left(\mathcal{K}_{A_{i}}\right) \cap[0,1]^{T}=\bigcap_{i=1}^{k} V\left(y_{M}, A_{i}\right),
\end{aligned}
$$

as desired and we have shown that $\mathcal{K}_{M} \subset \operatorname{pr}_{M}(\mathcal{K})$. Both inclusions give $\mathcal{K}_{M}=$ $\operatorname{pr}_{M}(\mathcal{K})$.

By definition, we have $\ell_{M}(x)=\sup \left\{\langle x, y\rangle: y \in \mathcal{K}_{M}\right\}$ for $x \in[0, \infty)^{M}$. Thus, 2.19 follows from $\mathcal{K}_{M}=\operatorname{pr}_{M}(\mathcal{K})$.

Finally, let $\mathcal{L} \subset[0, \infty)^{T}$ be also convex compact and satisfying 2.19 with $\mathcal{K}$ replaced by $\mathcal{L}$. Then it follows immediately that $\operatorname{pr}_{M}(\mathcal{L})=\mathcal{K}_{M}$ for any non-empty finite subset $M \subset T$. We conclude that $\mathcal{L} \subset \mathcal{K}$ by definition of $\mathcal{K}$. This finishes the proof.

In particular, the ECF $\theta$ of a simple max-stable process $X=\left\{X_{t}\right\}_{t \in T}$ can be expressed in terms of the dependency set $\mathcal{K}$ of $X$ as

$$
\theta(A)=\sup \left\{\sum_{t \in A} x_{t}: x \in \mathcal{K}\right\} .
$$

In order to make statements about the dependency sets $\mathcal{K}$ of processes $X=\left\{X_{t}\right\}_{t \in T}$ in terms of the ECF $\theta$, we introduce the following notation: For any non-empty finite subsets $A$ of $T$ we set the halfspace

$$
\mathcal{H}_{A}(\theta):=\left\{x \in[0, \infty)^{T}: \sum_{t \in A} x_{t} \leq \theta(A)\right\}
$$

that is bounded by the hyperplane

$$
\mathcal{E}_{A}(\theta):=\left\{x \in[0, \infty)^{T}: \sum_{t \in A} x_{t}=\theta(A)\right\} .
$$


Lemma 2.6.5. Let $\mathcal{K}$ be the dependency set of a simple max-stable process $X=$ $\left\{X_{t}\right\}_{t \in T}$ with ECF $\theta$. Then the following inclusion holds

$$
\mathcal{K} \subset \bigcap_{A \in \mathcal{F}(T) \backslash\{\emptyset\}} \mathcal{H}_{A}(\theta)
$$

On the other hand for each $A \in \mathcal{F}(T) \backslash\{\emptyset\}$ there is at least one point $\mathbf{x}^{\mathbf{A}}$ in the intersection

$$
\mathbf{x}^{\mathbf{A}} \in \mathcal{K} \cap \mathcal{E}_{A}(\theta)
$$

Proof. Let $A \in \mathcal{F}(T) \backslash\{\emptyset\}$ and $x \in \mathcal{K}$. Then the assumption $\sum_{t \in A} x_{t}>\theta(A)$ contradicts $\theta(A)=\sup \left\{\sum_{t \in A} x_{t}: x \in \mathcal{K}\right\}>\theta(A)$ (cf. $(2.20)$ ). So $\sum_{t \in A} x_{t} \leq \theta(A)$. This proves the inclusion. Secondly, since $\mathcal{K}$ is compact and the map $[0, \infty)^{T} \ni x \rightarrow$ $\sum_{t \in A} x_{t}$ is continuous, we know that it attains its supremum at some $\mathbf{x}^{\mathbf{A}} \in \mathcal{K}$.

Example 2.6.6. We give a simple multivariate example for Lemma 2.6.5 (illustrated in Figure 2.3 in the trivariate case): The Euclidean norm $\ell_{M}(x)=\|x\|_{2}$ is a stable tail dependence function on $[0, \infty)^{M}$ (cf. [66, Example 2]) and defines a simple maxstable distribution (cf. 1.2) with $\operatorname{ECF} \theta(A)=\sqrt{|A|}$ for $A \subset M$, such that

$$
\begin{aligned}
\mathcal{H}_{A}(\theta) & =\left\{x \in[0, \infty)^{M}:\left\langle x, \mathbf{1}_{A}\right\rangle \leq \sqrt{|A|}\right\} \\
\mathcal{E}_{A}(\theta) & =\left\{x \in[0, \infty)^{M}:\left\langle x, \mathbf{1}_{A}\right\rangle=\sqrt{|A|}\right\}
\end{aligned}
$$

for $\emptyset \neq A \subset M$. It can be easily seen that for $x \in[0, \infty)^{M} \backslash\left\{\mathbf{1}_{\emptyset}\right\}$

$$
\ell_{M}(x)=\|x\|_{2}=\left\langle x, x /\|x\|_{2}\right\rangle=\sup \left\{\langle x, y\rangle: y \in B^{+}\right\},
$$

where $B^{+}:=\left\{y \in[0, \infty)^{M}:\|y\|_{2} \leq 1\right\}$ denotes the positive part of the (Euclidean) unit ball. So, the dependency set $\mathcal{K}$ is clearly $B^{+}$in this case. Now, the planes $\mathcal{E}_{A}(\theta)$ are tangent to the boundary of $B^{+}$with common points $\mathbf{x}^{\mathbf{A}}=\mathbf{1}_{A} / \sqrt{|A|}$ for $\emptyset \neq A \subset M$, which makes it easy to see that Lemma 2.6.5 holds true in this example.

The following theorem shows that the inclusion from Lemma 2.6.5 is sharp and attained by the process from Theorem 2.3.5 b). Figure 2.4 illustrates a trivariate dependency set of this process. 


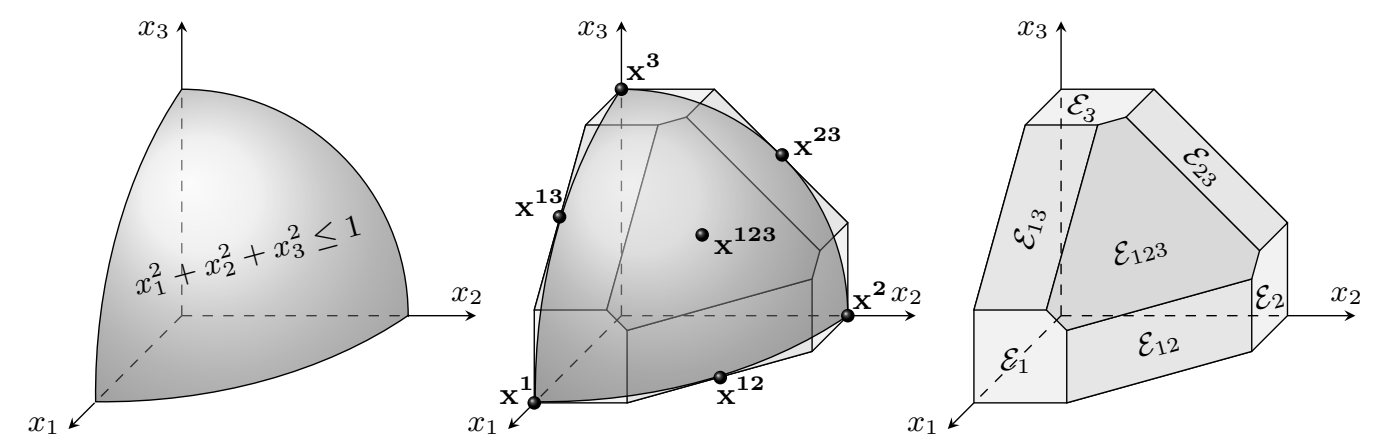

Figure 2.3.: The positive part of the Euclidean unit ball is the dependency set $\mathcal{K}$ of the simple max-stable distribution with stable tail dependence function the Euclidean norm $\ell(x)=\|x\|_{2}$ as illustrated here in the trivariate case. Indeed, $\mathcal{K}$ is contained in the intersection of halfspaces given by $\sum_{i \in A} x_{i} \leq \sqrt{|A|}=\theta(A)$. For each non-empty $A \subset\{1,2,3\}$ we have precisely one point $x^{A}=\mathbf{1}_{A} / \sqrt{|A|}$ in the intersection $\mathcal{K} \cap \mathcal{E}_{A}(\theta)$ (cf. Example 2.6.6 and Lemma 2.6.5.

Theorem 2.6.7. Let $\mathcal{K}^{*}$ be the dependency set of the simple max-stable process $X^{*}=\left\{X_{t}^{*}\right\}_{t \in T}$ from Theorem 2.3.5 b) with ECF $\theta$. Then

$$
\mathcal{K}^{*}=\bigcap_{A \in \mathcal{F}(T) \backslash\{\emptyset\}} \mathcal{H}_{A}(\theta) .
$$

Proof. First, we prove the theorem in the case, when $T=M$ is finite and $\mathcal{K}^{*}=\mathcal{K}_{M}^{*}$ : Therefore, write

$$
\mathcal{L}_{M}:=\bigcap_{\emptyset \neq A \subset M} \mathcal{H}_{A}(\theta)=\left\{x \in[0, \infty)^{M}:\left\langle x, \mathbf{1}_{A}\right\rangle \leq \theta(A) \text { for all } \emptyset \neq A \subset M\right\}
$$

The inclusion $\mathcal{K}_{M}^{*} \subset \mathcal{L}_{M}$ is proven in Lemma 2.6.5. So, it remains to show the other inclusion $\mathcal{L}_{M} \subset \mathcal{K}_{M}^{*}$. Due to 2.18 we have that

$$
\mathcal{K}_{M}^{*}=\bigcap_{x \in S_{M}}\left\{y \in[0, \infty)^{M}:\langle x, y\rangle \leq \ell_{M}^{*}(x)\right\},
$$

where

$$
\ell_{M}^{*}(x)=\sum_{\emptyset \neq L \subset M} \tau_{L}^{M} \bigvee_{t \in L} x_{t}
$$

is the stable tail dependence function of $\left\{X_{t}^{*}\right\}_{t \in M}$, here expressed in terms of the coefficients $\tau_{L}^{M}$ from Theorem 2.3.5 b (cf. 2.8). 
Thus, it suffices to show the following implication in order to prove $\mathcal{L}_{M} \subset \mathcal{K}_{M}^{*}$ :

$$
x \in S_{M} \text { and } y \in \mathcal{L}_{M} \quad \Longrightarrow \quad\langle x, y\rangle \leq \ell_{M}^{*}(x) .
$$

We now prove this implication: Without loss of generality, we may label the elements of $M=\left\{t_{1}, \ldots, t_{m}\right\}$ such that $x_{t_{1}} \geq x_{t_{2}} \geq \cdots \geq x_{t_{m}}$. Then we may write $x=$ $\left(x_{t}\right)_{t \in M} \in S_{M} \subset[0, \infty)^{M}$ as

$$
x=\underbrace{x_{t_{m}}}_{\geq 0} \mathbf{1}_{M}+\underbrace{\left(x_{t_{m-1}}-x_{t_{m}}\right)}_{\geq 0} \mathbf{1}_{M \backslash\left\{t_{m}\right\}}+\cdots+\underbrace{\left(x_{t_{2}}-x_{t_{3}}\right)}_{\geq 0} \mathbf{1}_{\left\{t_{1}, t_{2}\right\}}+\underbrace{\left(x_{t_{1}}-x_{t_{2}}\right)}_{\geq 0} \mathbf{1}_{\left\{t_{1}\right\}} .
$$

Taking the scalar product with $y \in \mathcal{L}_{M}$, we conclude

$$
\begin{aligned}
\langle x, y\rangle \leq x_{t_{m}} \theta(M)+\left(x_{t_{m-1}}-x_{t_{m}}\right) \theta\left(M \backslash\left\{t_{m}\right\}\right)+\ldots \\
\cdots+\left(x_{t_{2}}-x_{t_{3}}\right) \theta\left(\left\{t_{1}, t_{2}\right\}\right)+\left(x_{t_{1}}-x_{t_{2}}\right) \theta\left(\left\{t_{1}\right\}\right) \\
=x_{t_{m}}\left(\theta(M)-\theta\left(M \backslash\left\{t_{m}\right\}\right)\right)+\cdots+x_{t_{2}}\left(\theta\left(\left\{t_{1}, t_{2}\right\}\right)-\theta\left(\left\{t_{1}\right\}\right)\right)+x_{t_{1}} \theta\left(\left\{t_{1}\right\}\right) .
\end{aligned}
$$

On the other hand the stable tail dependence function $\ell_{M}^{*}$ is by this ordering of the components of $x$ given as

$$
\ell_{M}^{*}(x)=\sum_{\emptyset \neq L \subset M} \tau_{L}^{M} \bigvee_{t \in L} x_{t}=\sum_{i=1}^{m} x_{t_{i}}\left(\sum_{L \subset M: t_{1}, \ldots, t_{i-1} \notin L, t_{i} \in L} \tau_{L}^{M}\right) .
$$

From 2.15) we see that this expression coincides with the r.h.s. of 2.21$)$. Thus, we have our desired inequality $\langle x, y\rangle \leq \ell_{M}^{*}(x)$. This finishes the proof in the case, when $T=M$ is finite.

Otherwise, the definition of the dependency set $\mathcal{K}^{*}$ and the result for finite $M$ give

$$
\mathcal{K}^{*}=\bigcap_{M \in \mathcal{F}(T) \backslash\{\emptyset\}} \operatorname{pr}_{M}^{-1}\left(\mathcal{K}_{M}^{*}\right)=\bigcap_{M \in \mathcal{F}(T) \backslash\{\emptyset\}} \bigcap_{\emptyset \neq A \subset M} \operatorname{pr}_{M}^{-1}\left(\mathcal{H}_{A}^{M}(\theta)\right),
$$

where

$$
\mathcal{H}_{A}^{M}(\theta)=\left\{x \in[0, \infty)^{M}: \sum_{t \in A} x_{t} \leq \theta(A)\right\}
$$

Since $\operatorname{pr}_{M}^{-1}\left(\mathcal{H}_{A}^{M}(\theta)\right)=\mathcal{H}_{A}(\theta)$ for $\emptyset \neq A \subset M$, the claim follows. 


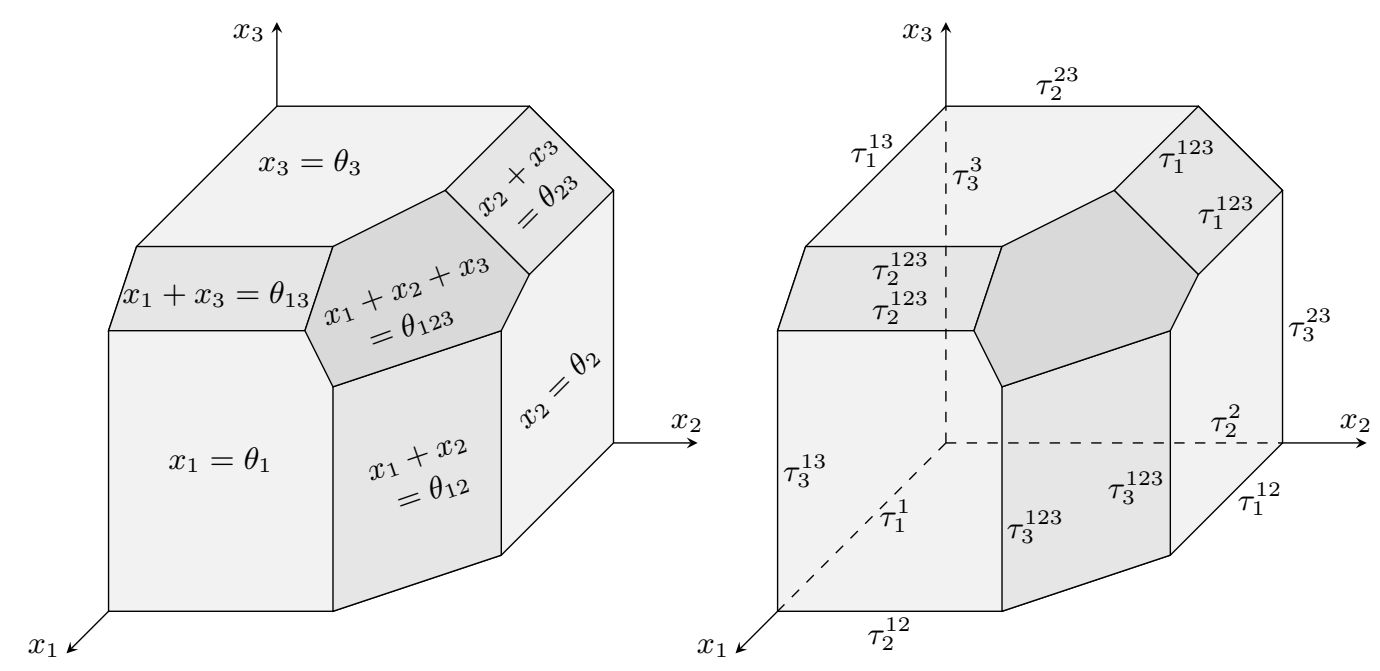

Figure 2.4.: Dependency set $\mathcal{K}^{*}$ of the random vector $\left\{X_{t}^{*}\right\}_{t \in M}$ for $M=\{1,2,3\}$. The dependency set $\mathcal{K}^{*}$ is bounded by the hyperplanes $\mathcal{E}_{A}(\theta)$ that are given by the equations $\sum_{t \in A} x_{t}=\theta(A)$, where $\theta$ denotes the ECF of $X^{*}$. The coefficients $\tau_{\{t\}}^{L}$ for $L \in \mathcal{F}(M) \backslash\{\emptyset\}$ and $t \in L$ turn up as lengths of the resulting polytope $\mathcal{K}^{*}$ (cf. Theorem 2.3.5 b) and Theorem 2.6.7.

So, if we fix the ECF $\theta$ of a simple max-stable process on $T$, then the process from Theorem 2.3.5 b yields a maximal dependency set $\mathcal{K}^{*}$ w.r.t. inclusion, that is

$$
\mathcal{K}^{*}=\bigcup_{\substack{\mathcal{K} \text { dependency set } \\ \text { with the same ECF as }}} \mathcal{K} .
$$

Now, inclusion of dependency sets corresponds to stochastic ordering in the following sense (cf. [66, p. 242]): If $\mathcal{K}^{\prime}$ and $\mathcal{K}^{\prime \prime}$ denote the dependency sets of the simple maxstable processes $X^{\prime}$ and $X^{\prime \prime}$ respectively, then $\mathcal{K}^{\prime} \subset \mathcal{K}^{\prime \prime}$ implies

$$
\mathbb{P}\left(X_{t}^{\prime} \leq x_{t}, t \in M\right) \geq \mathbb{P}\left(X_{t}^{\prime \prime} \leq x_{t}, t \in M\right) \quad \forall x \in[0, \infty)^{M}
$$

for all $M \in \mathcal{F}(T) \backslash\{\emptyset\}$. This leads to the following sharp inequality:

Corollary 2.6.8. Let $X=\left\{X_{t}\right\}_{t \in T}$ be a simple max-stable process with ECF $\theta$. Let $M$ be a non-empty finite subset of $T$. Then

$$
\mathbb{P}\left(X_{t} \leq x_{t}, t \in M\right) \geq \exp \left(-\sum_{\emptyset \neq L \subset M} \tau_{L}^{M} \bigvee_{t \in L} \frac{1}{x_{t}}\right) \quad \forall x \in[0, \infty)^{M}
$$

where the coefficients $\tau_{L}^{M}$ depend only on $\theta$ and can be computed as in Theorem 2.3.5 b). Equality holds for the process $X^{*}$ from Theorem 2.3.5 b). 
Example 2.6.9. Let us abbreviate $\eta_{A}:=\theta(A)-1$. In the bivariate case the inequality 2.23 reads as

$$
\begin{aligned}
\mathbb{P}\left(X_{s} \leq x_{s}, X_{t} \leq x_{t}\right) & \geq \exp \left(-\left[\frac{\eta_{s t}}{x_{s} \vee x_{t}}+\frac{1}{x_{s} \wedge x_{t}}\right]\right) \\
& =\exp \left(-\frac{\eta_{s t}+1}{x_{s} \wedge x_{t}}\right) \exp \left(\eta_{s t}\left|\frac{1}{x_{s}}-\frac{1}{x_{t}}\right|\right) .
\end{aligned}
$$

Indeed this inequality is much better then the trivial inequality

$$
\mathbb{P}\left(X_{s} \leq x_{s}, X_{t} \leq x_{t}\right) \geq \mathbb{P}\left(X_{s} \leq x_{s} \wedge x_{t}, X_{t} \leq x_{s} \wedge x_{t}\right)
$$

which can be written in the above terms as

$$
\mathbb{P}\left(X_{s} \leq x_{s}, X_{t} \leq x_{t}\right) \geq \exp \left(-\frac{\eta_{s t}+1}{x_{s} \wedge x_{t}}\right)
$$

Further note that $\eta_{s t}=\theta(\{s, t\})-1$ can be interpreted as a normalized madogram:

$$
\eta_{s t} \stackrel{\text { 2.14 }}{=} \lim _{x \rightarrow \infty} \frac{\mathbb{P}\left(X_{s} \geq x \text { or } X_{t} \geq x\right)}{\mathbb{P}\left(X_{t} \geq x\right)}-1=\lim _{x \rightarrow \infty} \frac{\mathbb{E}\left|\mathbb{1}_{X_{s} \geq x}-\mathbb{1}_{X_{t} \geq x}\right|}{2 \mathbb{E} \mathbb{1}_{X_{t} \geq x}} .
$$

If we additionally take into account that (cf. [85, (13)])

$$
\eta_{r s} \vee \eta_{s t} \vee \eta_{r t} \vee\left(\eta_{r s}+\eta_{s t}+\eta_{r t}-1\right) \leq \eta_{r s t} \leq\left(\eta_{r s}+\eta_{s t}\right) \wedge\left(\eta_{s t}+\eta_{r t}\right) \wedge\left(\eta_{r t}+\eta_{r s}\right),
$$

we obtain from 2.23 the following (sharp) inequality for the trivariate distribution of a simple max-stable random vector $\left(X_{r}, X_{s}, X_{t}\right)$ from bivariate extremal coefficients:

$$
\begin{aligned}
& \mathbb{P}\left(X_{r} \leq x_{r}, X_{s} \leq x_{s}, X_{t} \leq x_{t}\right) \\
& \geq \exp \left(-\left[\frac{1-\eta_{r s} \vee \eta_{s t} \vee \eta_{r t}}{x_{r} \wedge x_{s} \wedge x_{t}}+\left(a_{r s t} \wedge 1\right)\left(\frac{1}{x_{r} \wedge x_{s}}+\frac{1}{x_{s} \wedge x_{t}}+\frac{1}{x_{r} \wedge x_{t}}\right)\right.\right. \\
& \left.\left.-\left(\frac{\eta_{r s}}{x_{r} \wedge x_{s}}+\frac{\eta_{s t}}{x_{s} \wedge x_{t}}+\frac{\eta_{r t}}{x_{r} \wedge x_{t}}\right)+a_{r s t}\left(\frac{1}{x_{r}}+\frac{1}{x_{s}}+\frac{1}{x_{t}}\right)-\left(\frac{\eta_{s t}}{x_{r}}+\frac{\eta_{r t}}{x_{s}}+\frac{\eta_{r s}}{x_{t}}\right)\right]\right),
\end{aligned}
$$

where $a_{r s t}:=\left(\eta_{r s}+\eta_{s t}\right) \wedge\left(\eta_{r s}+\eta_{r t}\right) \wedge\left(\eta_{s t}+\eta_{r t}\right)$.

Remark 2.6.10. It is an open problem and it would be interesting to know whether there exist also minimal dependency sets in the sense of 2.22 and if they would help to better understand the classification of all dependency structures. In view of Lemma 2.6.5 and Theorem 2.6.7 a very naive idea would be to take one point from each of the sets $\mathcal{K}^{*} \cap \mathcal{E}_{A}$ where $A \in \mathcal{F}(T) \backslash\{\emptyset\}$ and then to take the convex hull with 0 included. However this fails to be a dependency set in dimensions $|T| \geq 3$, since it is not even a zonoid, which would be necessary (cf. [66]). 


\subsection{Directional ECFs}

By definition the ECF of a simple max-stable process $X$ on $T$ is bound to data on the diagonals in $[0, \infty)^{A}$ for finite subsets $A \subset T$. Inspired by [74], we now involve an additional direction $u$ that may vary in $(0, \infty)^{T}$ into the previous setup. We shall see that most results for standard ECFs as considered before (where $u=\mathbf{1}_{T}$ ) immediately transfer to directional ECFs as introduced below.

By slight abuse of notation we simply write $\ell_{M}(u)$ instead of $\ell_{M}\left(\operatorname{pr}_{M}(u)\right)$ for $u \in[0, \infty)^{T}$ omitting the projection onto $[0, \infty)^{M}$ which is already clear from the subindex of the stable tail dependence function $\ell_{M}$. Secondly, we abbreviate $u_{A}:=$ $u \cdot \mathbf{1}_{A}$ where $\mathbf{1}_{A}$ denotes the indicator function of a set $A \subset T$ and the product is meant componentwise. With this notation in mind we define the directional extremal coefficient function (directional ECF) $\theta^{(u)}$ of a simple max-stable process $X$ on $T$ for $u \in(0, \infty)$ as follows:

$$
\begin{aligned}
\theta^{(u)} & : \mathcal{F}(T) \longrightarrow[0, \infty) \\
\theta^{(u)}(A) & :=\left\{\begin{array}{ll}
\int_{S_{M}}\left(\bigvee_{t \in A} u_{t} a_{t}\right) H_{M}(\mathrm{~d} a)=\ell_{M}\left(u_{A}\right) & \emptyset \neq A \subset M \\
0 & A=\emptyset
\end{array},\right.
\end{aligned}
$$

where $H_{M}$ and $\ell_{M}$ are again the spectral measure and the stable tail dependence function of $\left\{X_{t}\right\}_{t \in M}$, respectively.

Since $\ell_{M}$ is homogeneous (of degree 1), the directional ECF $\theta^{(u)}$ is also homogeneous in the variable $u$. Apriori it is clear that

$$
\bigvee_{t \in A} u_{t} \leq \theta^{(u)}(A) \leq \sum_{t \in A} u_{t}
$$

if $A \neq \emptyset$. The set of all directional ECFs w.r.t. $u \in(0, \infty)^{T}$ will be denoted by

$$
\Theta^{(u)}(T):=\left\{\theta^{(u)}: \mathcal{F}(T) \rightarrow[0, \infty): \begin{array}{l}
\theta^{(u)} \text { is a directional ECF w.r.t. } u \\
\text { of a simple max-stable process on } T .
\end{array}\right\} .
$$

Following the steps taken for standard ECFs (cf. (2.7), we consider the following ansatz for a collection of spectral measures $\left\{H_{M}^{(u)}\right\}_{\emptyset \neq M \in \mathcal{F}(T)}$

$$
H_{M}^{(u)}:=\sum_{\emptyset \neq L \subset M} \tau_{L}^{M}(u)\left\|(1 / u)_{L}\right\| \delta_{(1 / u)_{L} /\left\|(1 / u)_{L}\right\|} \cdot
$$

The vector $(1 / u)_{L} \in[0, \infty)^{M}$ is meant in the sense of $(1 / u)_{L}(t)=1 / u$ for $t \in L$ and 
$(1 / u)_{L}(t)=0$ for $t \in M \backslash L$. If this collection is consistent, these spectral measures form the max-linear f.d.d. of a max-stable process $X^{(u)}$ that are given by

$$
-\log \mathbb{P}\left(X_{t}^{(u)} \leq x_{t}, t \in M\right)=\sum_{\emptyset \neq L \subset M} \tau_{L}^{M}(u) \bigvee_{t \in L} \frac{1}{u_{t} x_{t}}
$$

In matrix notation, i.e. $X_{t}^{(u)}=\bigvee_{\emptyset \neq L \subset M} a_{t, L}^{M}(u) Z_{L}$ for $t \in M$ with standard Fréchet variables $Z_{L}$ and a matrix $\left\{a_{t, L}^{M}(u)\right\}_{t, L}$ where $t$ ranges through $M$ and $L$ through all non-empty subsets of $M$, this distribution corresponds to the columns

$$
a_{L}^{M}(u)=\tau_{L}^{M}(u)(1 / u)_{L}
$$

Indeed, Lemma 2.2.1 generalizes as follows, where the proof transfers verbatim to the new situation.

Lemma 2.7.1. Let $T$ be an arbitrary set and $u \in(0, \infty)^{T}$. Let coefficients $\tau_{L}^{M}(u)$ be given for $M \in \mathcal{F}(T) \backslash\{\emptyset\}$ and $L \in \mathcal{F}(M) \backslash\{\emptyset\}$, such that

(i) $\tau_{L}^{M}(u) \geq 0$ for all $M \in \mathcal{F}(T) \backslash\{\emptyset\}$ and $L \in \mathcal{F}(M) \backslash\{\emptyset\}$,

(ii) $\tau_{L}^{M}(u)=\tau_{L}^{M \cup\{t\}}(u)+\tau_{L \cup\{t\}}^{M \cup\{t\}}(u)$ for all $M \in \mathcal{F}(T) \backslash\{\emptyset\}$ and $L \in \mathcal{F}(M) \backslash\{\emptyset\}$ and $t \in T \backslash M$,

(iii) $\tau_{\{t\}}^{\{t\}}(u)=u_{t}$ for all $t \in T$.

Then the spectral measures $\left\{H_{M}^{(u)}\right\}_{M \in \mathcal{F}(T) \backslash\{\emptyset\}}$ from 2.27) define a simple max-stable process $X^{(u)}=\left\{X_{t}^{(u)}\right\}_{t \in T}$ on $T$ with f.d.d. as in 2.28).

Correspondingly, we may characterize the set $\Theta^{(u)}(T)$ of directional ECFs and define for each directional ECF a realizing max-linear process $X^{(u)}$ as in Theorem 2.7.3. This time, we give an alternative proof for the negative definiteness (complete alternation) of directional ECFs as an immediate consequence of the following Proposition 2.7.2. We say that a function on $[0, \infty)^{M}$ is max-completely alternating, if it is completely alternating on the semigroup $\left([0, \infty)^{M}, \vee, \mathbf{1}_{\emptyset}\right)$ (cf. Definition A.1.6. Recall that if $u \in[0, \infty)^{M}$ and $A \subset M$, we denote $u_{A}:=u \cdot \mathbf{1}_{A}$, where the product is meant componentwise.

Proposition 2.7.2. Let $M$ be a non-empty finite set and $\ell:[0, \infty)^{M} \rightarrow \mathbb{R}$. For $u, x \in[0, \infty)^{M}$ define $\theta(u, x): \mathcal{F}(M) \rightarrow \mathbb{R}$ by $\theta(u, x)(A):=\ell\left(x+u_{A}\right)$. Then $\ell$ is max-completely alternating if and only if $\theta(u, x)$ is negative definite (completely alternating) on $\mathcal{F}(M)$ for all $u, x \in[0, \infty)^{M}$. 
Proof. Let $\ell$ be max-completely alternating. We need to establish 2.12 for $\psi=$ $\theta(u, x)$. Indeed we have for $\emptyset \neq L=\left\{t_{1}, t_{2}, \ldots, t_{l}\right\} \subset M$ that

$$
\begin{aligned}
\sum_{I \subset L}(-1)^{|I|} \theta(u, x)((M \backslash L) \cup I) & =\sum_{I \subset L}(-1)^{|I|} \ell\left(x+u_{(M \backslash L) \cup I}\right) \\
& =\sum_{I \subset L}(-1)^{|I|} \ell\left(\left(x+u_{M \backslash L}\right) \vee(x+u)_{I}\right) \leq 0
\end{aligned}
$$

due to Lemma A.5.2 for $y=x+u_{M \backslash L}$ and $v=x+u$.

Reversely, let $\theta(u, x)$ be completely alternating on $\mathcal{F}(T)$ for all $u, x \in[0, \infty)$. We need to establish A.5 for $v, y \in \mathbb{R}^{M}$ with $v \geq y$ and $\emptyset \neq L \subset M$. Indeed, we have

$$
\begin{aligned}
\sum_{I \subset L}(-1)^{|I|} \ell\left(y \vee v_{I}\right) & =\sum_{I \subset L}(-1)^{|I|} \ell\left(y+(v-y)_{I}\right) \\
& =\sum_{I \subset L}(-1)^{|I|} \theta\left((v-y)_{I}, y\right)((M \backslash L) \cup I) \leq 0,
\end{aligned}
$$

since $\theta\left((v-y)_{I}, y\right)$ is completely alternating on $\mathcal{F}(T)$.

Theorem 2.7.3. a) The function $\theta^{(u)}: \mathcal{F}(T) \rightarrow \mathbb{R}$ is the directional ECF of a simple max-stable process on $T$ w.r.t. $u \in(0, \infty)^{T}$ if and only if the following conditions are satisfied:

(i) $\theta^{(u)}$ is negative definite (completely alternating) on $\mathcal{F}(T)$,

(ii) $\theta^{(u)}(\emptyset)=0$,

(iii) $\theta^{(u)}(\{t\})=u_{t}$ for all $t \in T$.

b) If these conditions are satisfied, the following choice of coefficients

$$
\begin{aligned}
\tau_{L}^{M}(u):=-\Delta_{\left\{t_{1}\right\}} \ldots \Delta_{\left\{t_{l}\right\}} \theta^{(u)}(M \backslash L) & =\sum_{I \subset L}(-1)^{|I|+1} \theta^{(u)}((M \backslash L) \cup I) \\
& \forall M \in \mathcal{F}(T) \backslash\{\emptyset\}, \emptyset \neq L=\left\{t_{1}, \ldots, t_{l}\right\} \subset M
\end{aligned}
$$

for model 2.27) defines a simple max-stable process $X^{(u)}$ on $T$ which realizes $\theta^{(u)}$ as its own ECF.

Proof. The necessity of (ii) and (iii) is again immediate. In order to establish negative definiteness of $\theta^{(u)}$ note that this property is given by finite-dimensional inequalities such that it suffices to consider the case where $T=M$ is finite. But then it follows directly from the previous Proposition 2.7.2 when applied to the stable tail dependence function $\ell_{M}$ in the role of $\ell$ and considering $\theta^{(u)}=\theta\left(u, \mathbf{1}_{\emptyset}\right.$ ) (cf. Proposition A.5.1 for the max-complete alternation of $\ell_{M}$ ). 
Sufficiency of (i),(ii),(iii) by the construction of a realizing process $X^{(u)}$ can be achieved as before in the proof of Theorem 2.3.5 if $\theta$ is replaced by $\theta^{(u)}$ and $\tau_{L}^{M}$ by $\tau_{L}^{M}(u)$ when Lemma 2.7 .1 is applied.

\subsubsection{Consequences of negative definiteness}

We collect some immediate consequences from Theorem 2.7.3, now for directional ECFs analogously to Section 2.4. First, note that the set of all directional ECFs w.r.t. $u \in(0, \infty)^{T}$ (cf. 2.26) coincides with the set of standard ECFs in case we admit a different (fixed) scale at each location (cf. Remark 2.3.7)

$$
\Theta^{(u)}(T)=\left\{\theta: \mathcal{F}(T) \rightarrow \mathbb{R}: \begin{array}{l}
\theta \text { is negative definite (completely alternating) } \\
\text { on } \mathcal{F}(T), \theta(\emptyset)=0, \theta(\{t\})=u_{t} \text { for } t \in T .
\end{array}\right\}
$$

Corollary 2.7.4. The set of directional ECFs $\Theta^{(u)}(T)$ is convex and compact w.r.t. the topology of pointwise convergence.

Proof. Convexity is readily seen from 2.30, whereas compactness can be derived as in Corollary 2.4 .2 if we consider the bound 2.25 instead of $\theta(A) \in[1,|A|]$.

The operation of Bernstein functions from Corollary 2.4.8 transfers immediately as follows.

Corollary 2.7.5. Let $T$ be a set and $g$ be a Bernstein function which is not constant and satisfies $g(0)=0$. Then

$$
\theta^{(u)} \in \Theta^{(u)}(T) \Longrightarrow g \circ \theta^{(u)} \in \Theta^{(g \circ u)}(T) .
$$

An integral representation for directional ECFs $\theta^{(u)}$ on the Cantor cube and a spectral representation of the corresponding process $X^{(u)}$ may be formulated in analogy to Corollary 2.4.4 and Theorem 2.4.6.

Corollary 2.7.6. Let $\theta^{(u)} \in \Theta^{(u)}(T)$ be a directional ECF w.r.t. $u \in(0, \infty)^{T}$. Then $\theta^{(u)}$ uniquely determines a positive Radon measure $\mu^{(u)}$ on $\mathcal{P}(T) \backslash\{\emptyset\}$ such that

$$
\theta^{(u)}(A)=\mu^{(u)}(\{Q \in \mathcal{P}(T) \backslash\{\emptyset\}: A \cap Q \neq \emptyset\})=\int_{\mathcal{P}(T) \backslash\{\emptyset\}} \mathbb{1}_{A \cap Q \neq \emptyset} \mu^{(u)}(\mathrm{d} Q),
$$

where $\theta^{(u)}(\{t\})=u_{t}$ for $t \in T$. Moreover, the function $\theta^{(u)}$ is bounded if and only if $\mu^{(u)}(\mathcal{P}(T) \backslash\{\emptyset\})<\infty$. 
Theorem 2.7.7. Let $X^{(u)}=\left\{X_{t}^{(u)}\right\}_{t \in T}$ be the simple max-stable process from Theorem 2.7.3 b) with directional ECF $\theta^{(u)}$. Then $X^{(u)}$ has the following spectral representation $(\Omega, \mathcal{A}, \nu, V)$ :

- $(\Omega, \mathcal{A}, \nu)$ is the measure space $\left(\mathcal{P}(T), \mathcal{B}(\mathcal{P}(T)), \mu^{(u)}\right)$ from Corollary 2.7.6.

- $V_{t}(Q)=\frac{1}{u_{t}} \cdot \mathbb{1}_{t \in Q}$.

The respective proofs are almost verbatim repetitions of the proofs of the previous results involving Theorem 2.7.3 instead of Theorem 2.3.5 and 2.28) instead of (2.8) and are therefore omitted.

\subsubsection{Dependency sets}

Also in the generalized situation the dependency sets $\mathcal{K}^{(u)}$ of processes $X^{(u)}$ take an exceptional role among dependency sets of processes sharing the same directional ECF w.r.t. $u \in(0, \infty)^{T}$ as we shall see next. Because of 2.19 the directional ECF $\theta^{(u)}$ of a simple max-stable process $X$ can be expressed in terms of the dependency set $\mathcal{K}$ of $X$ as

$$
\theta^{(u)}(A)=\sup \left\{\sum_{t \in A} u_{t} x_{t}: x \in \mathcal{K}\right\} .
$$

We define the following halfspaces and hyperplanes for non-empty finite subsets $A$ of $T$ and $u \in(0, \infty)^{T}$

$$
\begin{aligned}
\mathcal{H}_{A}\left(\theta^{(u)}\right): & =\left\{x \in[0, \infty)^{T}: \sum_{t \in A} u_{t} x_{t} \leq \theta^{(u)}(A)\right\} \\
\mathcal{E}_{A}\left(\theta^{(u)}\right) & :=\left\{x \in[0, \infty)^{T}: \sum_{t \in A} u_{t} x_{t}=\theta^{(u)}(A)\right\}
\end{aligned}
$$

Note that $\mathcal{H}_{A}\left(\theta^{(u)}\right)$ and $\mathcal{E}_{A}\left(\theta^{(u)}\right)$ indeed only depend on $\theta^{(u)}$ since $u_{t}=\theta^{(u)}(\{t\})$. With these definitions we may adapt Lemma 2.6.5 and Theorem 2.6.7 as below to Lemma 2.7.8 and Theorem 2.7.10.

Exemplarily, we carry out the proof of Theorem 2.7.10 explicitly, whereas it suffices to replace $\sum_{t \in A} x_{t}$ by $\sum_{t \in A} u_{t} x_{t}, \theta(A)$ by $\theta^{(u)}(A)$ and 2.20 by 2.31 in the proof of Lemma 2.6.5 in order to obtain Lemma 2.7.8. 
Lemma 2.7.8. Let $\mathcal{K}$ be the dependency set of a simple max-stable process $X=$ $\left\{X_{t}\right\}_{t \in T}$ with directional ECF $\theta^{(u)}$ for $u \in(0, \infty)^{T}$. Then the following inclusion holds

$$
\mathcal{K} \subset \bigcap_{A \in \mathcal{F}(T) \backslash\{\emptyset\}} \mathcal{H}_{A}\left(\theta^{(u)}\right)
$$

On the other hand for each $A \in \mathcal{F}(T) \backslash\{\emptyset\}$ there is at least one point $\mathbf{x}^{(\mathbf{u} ; \mathbf{A})}$ in the intersection

$$
\mathbf{x}^{(\mathbf{u} ; \mathbf{A})} \in \mathcal{K} \cap \mathcal{E}_{A}\left(\theta^{(u)}\right)
$$

Example 2.7.9. As a simple example for Lemma 2.7.8 we explore Example 2.6.6 involving an additional direction $u \in(0, \infty)^{T}$. The example is illustrated in Figure 2.5 for the trivariate case. We have seen previously that the dependency set of the max-stable distribution with stable tail dependence function the Euclidean norm $\ell_{M}(x)=\|x\|_{2}$ is the positive part of the (Euclidean) unit ball $B^{+}:=\left\{y \in[0, \infty)^{M}\right.$ : $\left.\|y\|_{2} \leq 1\right\}$. The directional ECF of the corresponding max-stable distribution w.r.t. the direction $u \in(0, \infty)^{M}$ is thus given by $\theta^{(u)}(A)=\left\|u_{A}\right\|_{2}=\sqrt{\sum_{t \in A} u_{t}^{2}}$ for $A \subset M$, such that

$$
\begin{aligned}
\mathcal{H}_{A}\left(\theta^{(u)}\right) & =\left\{x \in[0, \infty)^{M}:\left\langle x, u_{A}\right\rangle \leq\left\|u_{A}\right\|_{2}\right\} \\
\mathcal{E}_{A}\left(\theta^{(u)}\right) & =\left\{x \in[0, \infty)^{M}:\left\langle x, u_{A}\right\rangle=\left\|u_{A}\right\|_{2}\right\}
\end{aligned}
$$

for $\emptyset \neq A \subset M$. Now, the planes $\mathcal{E}_{A}\left(\theta^{(u)}\right)$ are tangent to the boundary of $B^{+}$with common points $\mathbf{x}^{(\mathbf{u} ; \mathbf{A})}=u_{A} /\left\|u_{A}\right\|_{2}$ for $\emptyset \neq A \subset M$, which makes it easy to see that Lemma 2.7 .8 holds true in the example.

The following theorem shows that the inclusion from Lemma 2.7.8 is sharp and attained by the process from Theorem 2.7.3 b). Figure 2.6 illustrates a trivariate dependency set of this process.

Theorem 2.7.10. Let $\mathcal{K}^{(u)}$ be the dependency set of the simple max-stable process $X^{(u)}=\left\{X_{t}^{(u)}\right\}_{t \in T}$ from Theorem 2.7.3 b) with directional ECF $\theta^{(u)}$ for $u \in(0, \infty)^{T}$. Then

$$
\mathcal{K}^{(u)}=\bigcap_{A \in \mathcal{F}(T) \backslash\{\emptyset\}} \mathcal{H}_{A}\left(\theta^{(u)}\right)
$$




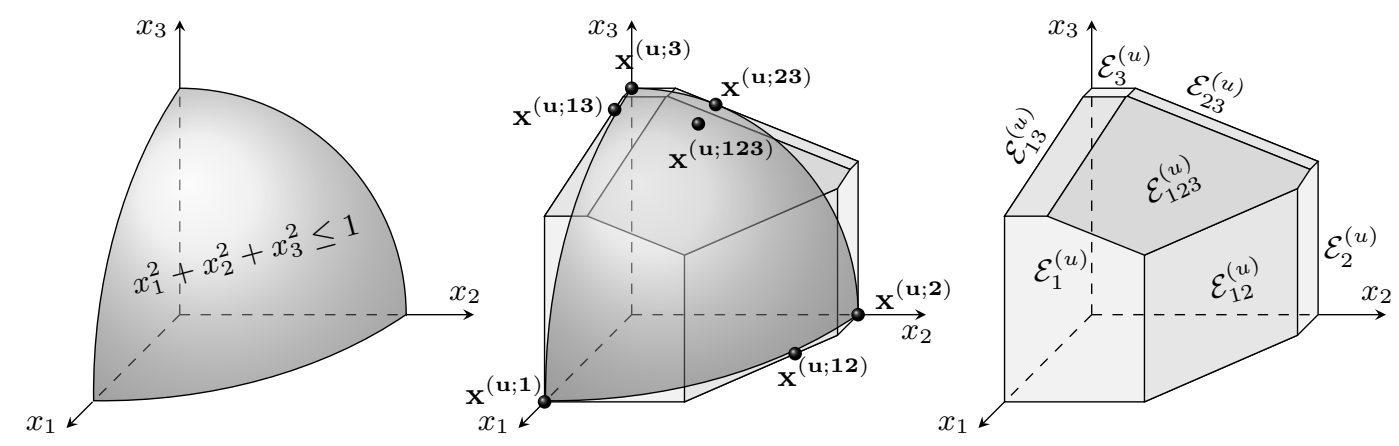

Figure 2.5.: The positive part of the Euclidean unit ball is the dependency set $\mathcal{K}$ of the simple max-stable distribution with stable tail dependence function the Euclidean norm $\ell(x)=\|x\|_{2}$ as illustrated here in the trivariate case. Indeed, $\mathcal{K}$ is contained in the intersection of halfspaces given by $\sum_{i \in A} u_{i} x_{i} \leq\left(\sum_{i \in A} u_{i}\right)^{1 / 2}=\theta^{(u)}(A)$. For each non-empty $A \subset\{1,2,3\}$ we have precisely one point $x^{(u ; A)}=u_{A} / \theta^{(u)}(A)$ in the intersection $\mathcal{K} \cap \mathcal{E}_{A}\left(\theta^{(u)}\right)$ (cf. Example 2.7.9 and Lemma 2.7.8.

Proof. Following the proof of Theorem 2.6.7 we start with the case where $T=M$ is finite and $\mathcal{K}^{(u)}=\mathcal{K}_{M}^{(u)}$ and abbreviate the r.h.s. by

$$
\mathcal{L}_{M}:=\bigcap_{\emptyset \neq A \subset M} \mathcal{H}_{A}\left(\theta^{(u)}\right)=\left\{x \in[0, \infty)^{M}:\left\langle x, u_{A}\right\rangle \leq \theta^{(u)}(A) \text { for all } \emptyset \neq A \subset M\right\} .
$$

The inclusion $\mathcal{K}_{M}^{(u)} \subset \mathcal{L}_{M}$ is proven in Lemma 2.7.8. In order to show the reverse inclusion $\mathcal{L}_{M} \subset \mathcal{K}_{M}^{(u)}$, we need to establish the following implication

$$
x \in S_{M} \text { and } y \in \mathcal{L}_{M} \quad \Longrightarrow \quad\langle x, y\rangle \leq \ell_{M}^{(u)}(x),
$$

where $\ell_{M}^{(u)}$ denotes the stable tail dependence function of $\left\{X_{t}^{(u)}\right\}_{t \in M}$ given by

$$
\ell_{M}^{(u)}(x)=\sum_{\emptyset \neq L \subset M} \tau_{L}^{M}(u) \bigvee_{t \in L} \frac{x_{t}}{u_{t}}
$$

in terms of the coefficients $\tau_{L}^{M}(u)$ from Theorem 2.7.3 b), cf. 2.28). We now prove this implication: Labelling the elements of $M=\left\{t_{1}, \ldots, t_{m}\right\}$ such that $\frac{x_{t_{1}}}{u_{t_{1}}} \geq \frac{x_{t_{2}}}{u_{t_{2}}} \geq$ $\cdots \geq \frac{x_{t_{m}}}{u_{t_{m}}}$ we may write $x=\left(x_{t}\right)_{t \in M} \in S_{M} \subset[0, \infty)^{M}$ as

$$
x=\underbrace{\frac{x_{t_{m}}}{u_{t_{m}}}}_{\geq 0} u_{M}+\underbrace{\left(\frac{x_{t_{m-1}}}{u_{t_{m-1}}}-\frac{x_{t_{m}}}{u_{t_{m}}}\right)}_{\geq 0} u_{M \backslash\left\{t_{m}\right\}}+\cdots+\underbrace{\left(\frac{x_{t_{1}}}{u_{t_{1}}}-\frac{x_{t_{2}}}{u_{t_{2}}}\right)}_{\geq 0} u_{\left\{t_{1}\right\}} .
$$



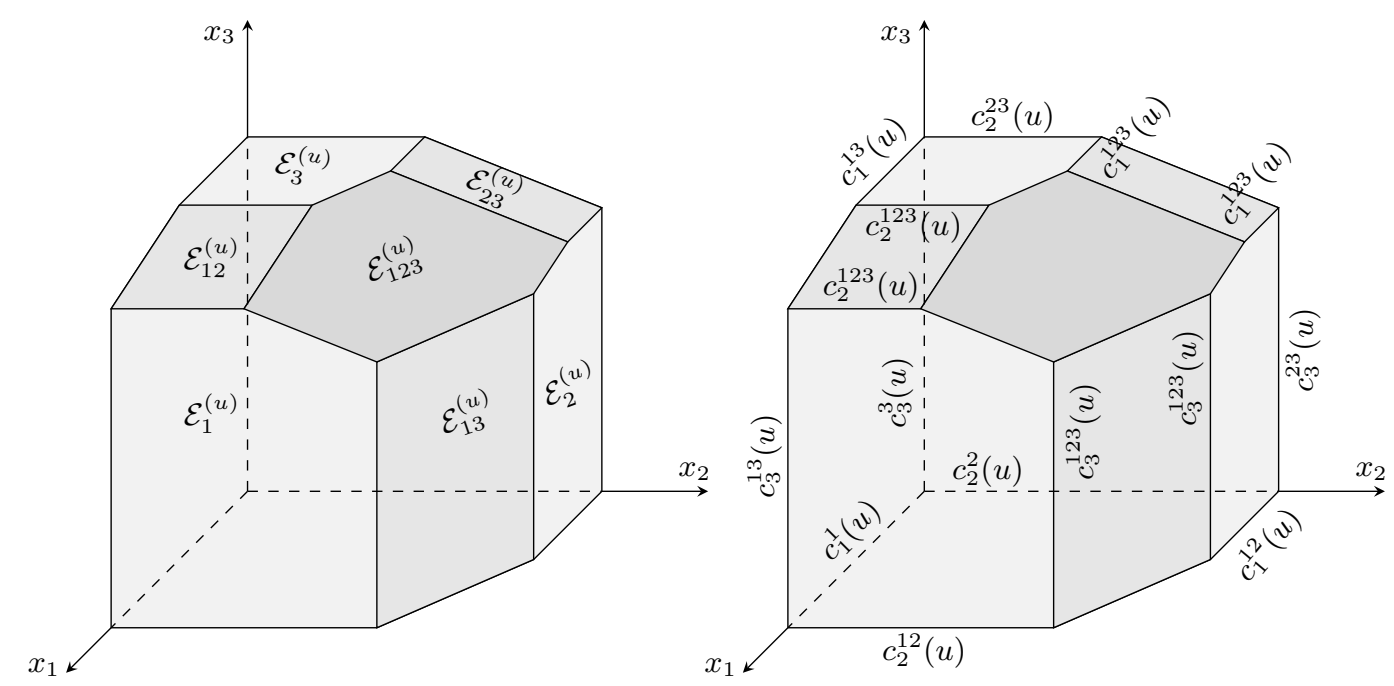

Figure 2.6.: Dependency set $\mathcal{K}^{(u)}$ of the random vector $\left\{X_{t}^{(u)}\right\}_{t \in M}$ for $M=\{1,2,3\}$. The dependency set $\mathcal{K}^{(u)}$ is bounded by the hyperplanes $\mathcal{E}_{A}\left(\theta^{(u)}\right)$ that are given by the equations $\sum_{t \in A} u_{t} x_{t}=\theta^{(u)}(A)$, where $\theta^{(u)}$ denotes the directional ECF of $X^{(u)}$. The coefficients $c_{t}^{L}(u):=\left(a_{\{t\}}^{L}(u)\right)_{t}=\tau_{\{t\}}^{L}(u) / u_{t}$ for $L \in \mathcal{F}(M) \backslash\{\emptyset\}$ and $t \in L$ (cf. $\left.2.29 p\right)$ turn up as lengths of the resulting polytope $\mathcal{K}^{(u)}$ (cf. Theorem 2.7.3 b) and Theorem 2.7.10p.

Taking the scalar product with $y \in \mathcal{L}_{M}$, we conclude

$$
\begin{aligned}
\langle x, y\rangle \leq & \frac{x_{t_{m}}}{u_{t_{m}}} \theta^{(u)}(M)+\left(\frac{x_{t_{m-1}}}{u_{t_{m-1}}}-\frac{x_{t_{m}}}{u_{t_{m}}}\right) \theta^{(u)}\left(M \backslash\left\{t_{m}\right\}\right)+\ldots \\
& \cdots+\left(\frac{x_{t_{2}}}{u_{t_{2}}}-\frac{x_{t_{3}}}{u_{t_{3}}}\right) \theta^{(u)}\left(\left\{t_{1}, t_{2}\right\}\right)+\left(\frac{x_{t_{1}}}{u_{t_{1}}}-\frac{x_{t_{2}}}{u_{t_{2}}}\right) \theta^{(u)}\left(\left\{t_{1}\right\}\right) \\
= & \frac{x_{t_{m}}}{u_{t_{m}}}\left(\theta^{(u)}(M)-\theta^{(u)}\left(M \backslash\left\{t_{m}\right\}\right)\right)+\cdots+\frac{x_{t_{2}}}{u_{t_{2}}}\left(\theta^{(u)}\left(\left\{t_{1}, t_{2}\right\}\right)-\theta^{(u)}\left(\left\{t_{1}\right\}\right)\right) \\
& +\frac{x_{t_{1}}}{u_{t_{1}}} \theta^{(u)}\left(\left\{t_{1}\right\}\right) .
\end{aligned}
$$

On the other hand the stable tail dependence function $\ell_{M}^{(u)}$ is by this ordering of the components of $\frac{x}{u}$ given as

$$
\ell_{M}^{(u)}(x)=\sum_{\emptyset \neq L \subset M} \tau_{L}^{M}(u) \bigvee_{t \in L} \frac{x_{t}}{u_{t}}=\sum_{i=1}^{m} \frac{x_{t_{i}}}{u_{t_{i}}}\left(\sum_{L \subset M: t_{1}, \ldots, t_{i-1} \notin L, t_{i} \in L} \tau_{L}^{M}(u)\right),
$$

which coincides with the r.h.s. of 2.32 and the desired inequality $\langle x, y\rangle \leq \ell_{M}^{(u)}(x)$ is shown. This finishes the proof for the case that $T=M$ is finite. If $T$ is infinite, apply the following replacements to the remaining proof of Theorem 2.6.7: $\mathcal{K}^{*}$ by $\mathcal{K}^{(u)}, \mathcal{K}_{M}^{*}$ by $\mathcal{K}_{M}^{(u)}, \theta$ by $\theta^{(u)}, \sum_{t \in A} x_{t}$ by $\sum_{t \in A} u_{t} x_{t}$. 
So, if we fix the directional ECF $\theta^{(u)}$ of a simple max-stable process on $T$ for $u \in(0, \infty)^{T}$, then the process from Theorem 2.7.3 b yields a maximal dependency set $\mathcal{K}^{(u)}$ w.r.t. inclusion, that is

$$
\mathcal{K}^{(u)}=\bigcup_{\substack{\mathcal{K} \text { dependency set } \\ \text { with the same directional } \\ \text { ECF w.r.t. } u \text { as } \mathcal{K}^{(u)}}} \mathcal{K} .
$$

As before, this implies the following sharp inequality.

Corollary 2.7.11. Let $X=\left\{X_{t}\right\}_{t \in T}$ be a simple max-stable process with directional ECF $\theta^{(u)}$ for some $u \in(0, \infty)^{T}$. Let $M$ be a non-empty finite subset of $T$. Then

$$
\mathbb{P}\left(X_{t} \leq x_{t}, t \in M\right) \geq \exp \left(-\sum_{\emptyset \neq L \subset M} \tau_{L}^{M}(u) \bigvee_{t \in L} \frac{1}{u_{t} x_{t}}\right) \quad \forall x \in[0, \infty)^{M}
$$

where the coefficients $\tau_{L}^{M}(u)$ depend only on $\theta^{(u)}$ and can be computed as in Theorem 2.7.3 b). Equality holds for the process $X^{(u)}$ from Theorem 2.7.3 b).

Example 2.7.12. We abbreviate $\theta_{s t}^{(u)}:=\theta^{(u)}(\{s, t\})$ for the bivariate case, such that the inequality 2.34 reads as

$$
\begin{aligned}
& \mathbb{P}\left(X_{s} \leq x_{s}, X_{t} \leq x_{t}\right) \geq \exp \left(-\left[\frac{\theta_{s t}^{(u)}-\left(u_{s}+u_{t}\right)}{u_{s} x_{s} \vee u_{t} x_{t}}+\frac{1}{x_{s}}+\frac{1}{x_{t}}\right]\right) \\
& =\exp \left(-\frac{\theta_{s t}^{(u)}}{u_{s} x_{s} \wedge u_{t} x_{t}}\right) \exp \left(\left[\theta_{s t}^{(u)}-\left(u_{s} \mathbb{1}_{u_{s} x_{s}<u_{t} x_{t}}+u_{t} \mathbb{1}_{u_{s} x_{s}>u_{t} x_{t}}\right)\right]\left|\frac{1}{u_{s} x_{s}}-\frac{1}{u_{t} x_{t}}\right|\right) .
\end{aligned}
$$

Indeed this inequality is much better then the trivial inequality

$$
\mathbb{P}\left(X_{s} \leq x_{s}, X_{t} \leq x_{t}\right) \geq \mathbb{P}\left(X_{s} \leq x_{s} \wedge x_{t}, X_{t} \leq x_{s} \wedge x_{t}\right)
$$

which can be written in the above terms as

$$
\mathbb{P}\left(X_{s} \leq x_{s}, X_{t} \leq x_{t}\right) \geq \exp \left(-\frac{\theta_{s t}^{(u)}}{u_{s} x_{s} \wedge u_{t} x_{t}}\right) .
$$





\section{A characterization of tail correlation functions}

This chapter is partially based on the arXiv-manuscript [93].

\subsection{Tail correlation functions}

Let $X=\left\{X_{t}\right\}_{t \in T}$ be a (not necessarily max-stable) stochastic process on a set $T$, such that $X$ has identical non-degenerate one-dimensional marginal distributions with upper endpoint $x^{*}$. Assuming that the limits of conditional probabilities

$$
\chi^{(X)}(s, t):=\lim _{x \rightarrow x^{*}} \mathbb{P}\left(X_{s}>x \mid X_{t}>x\right),
$$

exist, the function $\chi^{(X)}: T \times T \rightarrow[0,1]$ will be called the tail correlation function (TCF) of the process $X$. Roughly speaking, $\chi^{(X)}(s, t)$ expresses the probability of an extremal event at location $s$ given an extremal event at location $t$. The value 1 can be interpreted as total dependence in the tail and the value 0 as tail independence of $X_{s}$ and $X_{t}$.

Dating back to [32, 89, 95] the TCF is one of the most popular bivariate extremal dependence measures that has entered the literature under various names, most prominently (upper) tail dependence coefficient [4, 14, 28], $\chi$-measure [4, 10] or extremal coefficient function [29] (not to be confused with the ECF from the previous Chapter 2, Section 2.1. We prefer the name "tail correlation function", since $\chi^{(X)}$ measures tail dependence and $\chi^{(X)}$ is indeed a correlation function, i.e. a symmetric positive definite kernel on $T \times T$ with $\chi^{(X)}(t, t)=1$ for $t \in T$. This has been already 
observed in [14, 29, 86] and can be easily seen by writing

$$
\chi^{(X)}(s, t)=\lim _{x \rightarrow x^{*}} \frac{\mathbb{E}\left(\mathbb{1}_{X_{s}>x} \mathbb{1}_{X_{t}>x}\right)}{\mathbb{E}\left(\mathbb{1}_{X_{t}>x}\right)}=\lim _{x \rightarrow x^{*}} \frac{\operatorname{Cov}\left(\mathbb{1}_{X_{s}>x}, \mathbb{1}_{X_{t}>x}\right)}{\mathbb{P}\left(X_{t}>x\right)}+\lim _{x \rightarrow x^{*}} \mathbb{P}\left(X_{t}>x\right),
$$

where the constant term $\lim _{x \rightarrow x^{*}} \mathbb{P}\left(X_{t}>x\right)$ vanishes if $X$ has no mass at its upper endpoint $x^{*}$. Even though TCFs are non-negative correlation functions, beware that not all non-negative correlation functions are TCFs. For instance, $\eta:=1-\chi^{(X)}$ has to satisfy the triangle inequality (cf. (3.14)

$$
\eta(s, t) \leq \eta(s, r)+\eta(r, t) \quad r, s, t \in T .
$$

In the context of $\{0,1\}$-valued stochastic processes (or two-phase random media), it is well-known that the respective covariance functions obey this triangle inequality and implications are addressed e.g. in [45, 58, 60, see also [86] in the context of maxstable processes. We will compare the classes of TCFs and non-negative correlation functions in more detail in Section 3.6.

Further, note that TCFs are invariant under non-decreasing, eventually continuous marginal transformations, i.e.

$$
\chi^{(X)}(s, t)=\chi^{(f \circ X)}(s, t)
$$

if $f$ is such an allowed transformation. In particular, it is not important to which marginal distribution we standardize. If $X$ is stationary max-stable, the TCF $\chi^{(X)}$ contains also mixing information (cf. [50]). Estimators can be found in [90] (raw estimates), [86] (respecting positive definiteness) and [11, 69] (using a madogram approach).

Example 3.1.1. The simplest TCFs are the functions $\chi(s, t)=\delta_{s t}:=\mathbb{1}_{s=t}$ corresponding to a process of independent random variables, and the constant function $\chi(s, t)=1$ corresponding to a process of identical random variables. If $X$ is a Gaussian process on $T$, whose correlation function $\rho$ on $T \times T$ attains the value 1 only on the diagonal $\{(t, t): t \in T\}$, then its TCF will also be $\chi^{(X)}(s, t)=\delta_{s t}$, cf. [89, Theorem 3].

While Gaussian processes do not exhibit tail dependence, the class of max-stable processes naturally forms a class of processes for which the TCF is well-defined and yields rich classes of non-trivial examples as we shall see next and subsequently in Chapter 4. 


\subsection{TCFs are realized by max-stable processes}

Lemma 3.2.1. The TCF $\chi^{(X)}$ of a simple max-stable process $X$ on $T$ with spectral representation $(\Omega, \mathcal{A}, \nu, V)$ is well-defined and given by

$$
\chi^{(X)}(s, t)=\int_{\Omega} V_{s}(\omega) \wedge V_{t}(\omega) \nu(\mathrm{d} \omega) .
$$

Proof. The upper endpoint of the Fréchet distribution is $x^{*}=\infty$. An application of l'Hôpitals rule (cf. Lemma A.5.5) yields

$$
\begin{aligned}
\chi^{(X)}(s, t) & =\lim _{x \rightarrow \infty} \frac{\left(1-\mathbb{P}\left(X_{s} \leq x\right)\right)+\left(1-\mathbb{P}\left(X_{t} \leq x\right)\right)-\left(1-\mathbb{P}\left(X_{s} \leq x, X_{t} \leq x\right)\right)}{1-\mathbb{P}\left(X_{t} \leq x\right)} \\
& =\lim _{x \rightarrow \infty} \frac{-\log \mathbb{P}\left(X_{s} \leq x\right)-\log \mathbb{P}\left(X_{t} \leq x\right)+\log \mathbb{P}\left(X_{s} \leq x, X_{t} \leq x\right)}{-\log \mathbb{P}\left(X_{t} \leq x\right)} \\
& =\int_{\Omega} V_{s}(\omega)+V_{t}(\omega)-V_{s}(\omega) \vee V_{t}(\omega) \nu(\mathrm{d} \omega)=\int_{\Omega} V_{s}(\omega) \wedge V_{t}(\omega) \nu(\mathrm{d} \omega) .
\end{aligned}
$$

So simple max-stable processes form a class of processes, where TCFs always exist. Some non-trivial examples of TCFs are given below. Additionally, we refer to Chapter 4 for various concrete examples of TCFs stemming from max-stable processes.

Example 3.2.2 (Mixed Moving Maxima processes (M3)). Because of (3.2) the TCF $\chi^{(X)}$ of a general M3 process $X$ as in Example 1.2.1 can be computed as

$$
\chi^{(X)}(s, t)=\int_{\mathbb{F}} \int_{\mathbb{R}^{d}} f(z) \wedge f(z-(s-t)) \mathrm{d} z \mu(\mathrm{d} f) .
$$

In case $\mu$ is a weighted point mass at an indicator function $f=\mathbf{1}_{A}$, the TCF will be given by $\chi^{(X)}(\{s, t\})=\mathbf{1}_{A} * \mathbf{1}_{\check{A}}(s-t) / \nu_{d}(A)$, where $\mathbf{1}_{A} * \mathbf{1}_{\check{A}}$ means the convolution of $\mathbf{1}_{A}$ and $\mathbf{1}_{\check{A}}$ (with $\check{A}=\{-a: a \in A\}$ ) and $\nu_{d}(A)$ is the $d$-dimensional volume of $A$.

Example 3.2.3 (Brown-Resnick processes). In [52, Remark 25] the TCF $\chi^{(X)}$ of a Brown-Resnick process $X$ as in Example 1.2.3 has been identified as

$$
\chi^{(X)}(s, t)=\operatorname{erfc}\left(\sqrt{\frac{\gamma(s-t)}{8}}\right),
$$

where $\operatorname{erfc}(x)=2 / \sqrt{\pi} \int_{x}^{\infty} e^{-t^{2}} d t$ denotes the complementary error function and $\gamma$ the variogram of $X$.

It is natural to ask whether even further TCFs will arise if we do not restrict ourselves to the max-stable class, i.e. whether the set of TCFs stemming from max- 
stable processes is properly contained in the set of all TCFs or if these sets are equal. Therefore, let us denote the set of all TCFs and certain subclasses as follows:

$$
\begin{aligned}
\operatorname{TCF}(T) & :=\left\{\chi^{(X):} \begin{array}{l}
X \text { a stochastic process on } T \text { with identical } \\
\text { one-dimensional marginals such that } \chi^{(X)} \text { exists }
\end{array}\right\}, \\
\operatorname{TCF}_{\infty}(T) & :=\left\{\chi^{(X)} \in \operatorname{TCF}(T): X \text { with essential supremum } \infty\right\} \\
\operatorname{MAX}(T) & :=\left\{\chi^{(X)} \in \operatorname{TCF}(T): X \text { simple max-stable }\right\} .
\end{aligned}
$$

Here, $\mathrm{TCF}_{\infty}(T)$ represents the TCFs of processes whose marginals have no jump at the upper endpoint. Apriori it is clear that

$$
\operatorname{MAX}(T) \subset \operatorname{TCF}_{\infty}(T) \subset \operatorname{TCF}(T)
$$

Further, let us introduce the class of uncentered and normalized covariance functions of binary processes

$$
\operatorname{BIN}(T):=\left\{(s, t) \mapsto \mathbb{P}\left(Y_{s}=1 \mid Y_{t}=1\right): \begin{array}{l}
Y \text { a stochastic process on } T \text { with } \\
\text { identical one-dimensional marginals } \\
\text { with values in }\{0,1\} \text { and } \mathbb{E} Y_{t} \neq 0
\end{array}\right\},
$$

which is closely related to the above classes. By definition of $\mathrm{TCF}(T)$ and considering the processes $Y_{t}=\mathbb{1}_{Z_{t}>x}$ indexed by $x>0$, we observe

$$
\operatorname{TCF}(T) \subset \text { sequential closure of } \operatorname{BIN}(T),
$$

where the sequential closure is meant w.r.t. pointwise convergence. In the sequel, we will prove the following theorem, which gives an affirmative answer to the question whether $\operatorname{TCF}(T)$ and $\operatorname{MAX}(T)$ coincide and which involves also the connection to $\mathrm{TCF}_{\infty}(T)$ and $\mathrm{BIN}(T)$.

Theorem 3.2.4. a) For arbitrary sets $T$ we have the following coincidence

$$
\begin{aligned}
\operatorname{TCF}(T) & =\operatorname{TCF}_{\infty}(T)=\operatorname{MAX}(T) \\
& =\text { sequential closure of } \operatorname{BIN}(T)=\text { closure of } \operatorname{BIN}(T),
\end{aligned}
$$

where the (sequential) closure is meant w.r.t. pointwise convergence.

b) For infinite sets $T$ the inclusion $\operatorname{BIN}(T) \subsetneq \operatorname{TCF}(T)$ is proper.

c) For finite sets $M$, the equality $\operatorname{BIN}(M)=\operatorname{TCF}(M)$ holds. 
Remark 3.2.5. The class described in part a of Theorem 3.2.4 also comprises the extremogram $\rho_{A A}$ from [14] where $\mathbb{R}^{k}$-valued processes are considered (and not only real valued processes).

\subsubsection{Proof of Theorem 3.2 .4 using ECFs}

In view of (3.3) and (3.5) the gaps that need to be filled in for proving Theorem 3.2 .4 are the following two propositions:

Proposition 3.2.6. a) For arbitrary sets $T$ we have $\operatorname{BIN}(T) \subset \operatorname{MAX}(T)$.

b) For infinite sets $T$ the inclusion $\operatorname{BIN}(T) \subsetneq \operatorname{MAX}(T)$ is proper.

c) For finite sets $M$ the equality $\operatorname{BIN}(M)=\operatorname{MAX}(M)$ holds.

Proposition 3.2.7. $\operatorname{MAX}(T)$ is compact w.r.t. the toplogy of pointwise convergence.

The crucial point in establishing Propositions 3.2.6 and 3.2.7 is the close connection of the tail correlation function $(\mathrm{TCF}) \chi^{(X)}$ of a simple max-stable process $X=\left\{X_{t}\right\}_{t \in T}$ to the extremal coefficient function (ECF) $\theta^{(X)}$ of the respective process $X$. It follows easily from $(3.2)$ and $(2.3)$ that

$$
\chi^{(X)}(s, t)=2-\theta^{(X)}(\{s, t\}) .
$$

if $X$ is a simple max-stable process. Therefore, it will be convenient to introduce the following map

$$
\Psi:[0, \infty)^{\mathcal{F}(T)} \rightarrow[0, \infty)^{T \times T}, \quad \Psi(F)(s, t):=2-F(\{s, t\}),
$$

such that 3.6 reads as $\chi^{(X)}=\Psi\left(\theta^{(X)}\right)$. The set $\operatorname{MAX}(T)$ may thus be written as

$$
\operatorname{MAX}(T)=\Psi(\Theta(T))
$$

where $\Theta(T) \subset[0, \infty)^{\mathcal{F}(T)}$ denotes the set of all ECFs on $\mathcal{F}(T)$ (cf. 2.2 ). Similarly, $\operatorname{BIN}(T)$ is the image of bounded elements of $\Theta(T)$ that will be denoted by

$$
\Theta_{b}(T)=\{\theta \in \Theta(T): \theta \text { is bounded. }\} .
$$

Lemma 3.2.8. $\operatorname{BIN}(T)=\Psi\left(\Theta_{b}(T)\right)$. 
Proof. Let $f \in \operatorname{BIN}(T)$ and let $Y$ be a corresponding process with values in $\{0,1\}$ as in the definition of $\operatorname{BIN}(T)$ (cf. (3.4)). Let $C$ denote the capacity functional of $Y$ as in Example 2.3.4. Then $C(\{t\})=\mathbb{E} Y_{t}$ lies in the interval $(0,1]$ and is independent of $t \in T$ due to identical one-dimensional marginals. Further, note that the function $f$ is given by $f(s, t)=\mathbb{P}\left(Y_{s}=1 \mid Y_{t}=1\right)=2-C(\{s, t\}) / C(\{t\})$. Now, set $\theta(A):=$ $C(A) / C(\{t\})$ for $A \in \mathcal{F}(T)$. Then $\theta$ satisfies $\Psi(\theta)(s, t)=2-\theta(\{s, t\})=f(s, t)$ and $\theta$ is clearly bounded by $1 / C(\{t\})$. It follows from Example 2.3.4 and Theorem 2.3.5 that $\theta$ lies in $\Theta(T)$. Hence, $f \in \Psi\left(\Theta_{b}(T)\right)$.

Conversely, let $\theta \in \Theta_{b}(T)$ be bounded, say by $K$. Clearly, $K \geq \theta(\{t\})=1$. Set $C(A):=\theta(A) / K$. Then $C$ satisfies all requirements of Example 2.3.4 to define a binary process $Y$ with values in $\{0,1\}$, whose capacity functional equals $C$. The process $Y$ has identical one-dimensional marginals since $\theta(\{t\})=1$ for $t \in T$, and $\mathbb{E} Y_{t}=1 / K>0$. So $Y$ fulfills the requirements of a process in the definition of $\operatorname{BIN}(T)$ (cf. (3.4)). Finally, note that the corresponding function in $\operatorname{BIN}(T)$ is given by $\mathbb{P}\left(Y_{s}=1 \mid Y_{t}=1\right)=2-C(\{s, t\}) / C(\{t\})=\Psi(\theta)(s, t)$ as desired.

Now we can prove Propositions 3.2 .6 and 3.2 .7 and finally Theorem 3.2 .4

Proof of Proposition 3.2.6. a) For arbitrary sets $T$ the assertion $\operatorname{BIN}(T) \subset \operatorname{MAX}(T)$ follows directly from $(3.8)$ and Lemma 3.2.8.

b) Let $T$ be an infinite set and let $\chi(s, t):=\delta_{s t}$ as in Example 3.1.1. Indeed $\chi$ is an element of $\operatorname{MAX}(T)$ realized by the simple max-stable process $X$ on $T$, where the variables $\left\{X_{t}\right\}_{t \in T}$ are i.i.d. standard Fréchet random variables. Moreover, the only (!) preimage of $\chi$ under the map $\Psi$ (cf. (3.8) ) is the ECF $\theta(A)=|A|$. This can be seen as follows: Either an induction on the number of elements $|A|$ yields this result from the inequalities 2.12 . Or we can use the fact that pairwise independence of the components of a simple max-stable random vector implies their joint independence (cf. [4, p. 266] or [66, Proposition 7]). Either way, the ECF $\theta(A)=|A|$ is unbounded in case $T$ is an infinite set. Because of Lemma 3.2 .8 the inclusion $\operatorname{BIN}(T) \subsetneq \operatorname{MAX}(T)$ must be proper.

c) If $M$ is finite, take additionally into account that elements $\theta \in \Theta(M)$ are automatically bounded by $|M|$ and thus $\Theta(M)=\Theta_{b}(M)$.

Proof of Proposition 3.2.7. We use the description (3.8) of $\operatorname{MAX}(T)$. Note that the involved map $\Psi$ is continuous if we equip both spaces $[0, \infty)^{\mathcal{F}(T)}$ and $[0, \infty)^{T \times T}$ with the topology of pointwise convergence. So, $\operatorname{MAX}(T)$ is the continuous image of the compact space $\Theta(T)$ (cf. Corollary 2.4.2, and hence compact. 
Proof of Theorem 3.2.4 from Propositions 3.2.6 and 3.2.7. a) The assertion follows from

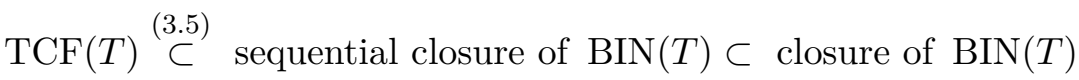

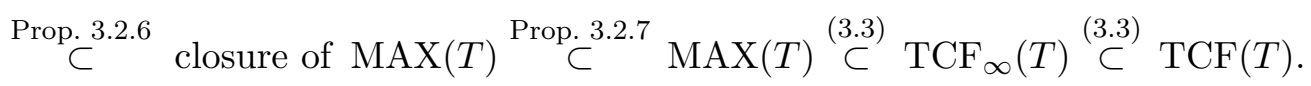

b) If $|T|$ is infinite, the inclusion $\operatorname{BIN}(T) \subsetneq \operatorname{MAX}(T)$ is proper, cf. Proposition 3.2.6.

c) For finite sets $M$ use that $\operatorname{BIN}(M)=\operatorname{MAX}(M)$ as shown in Proposition 3.2.6

Remark 3.2.9. The proof of Theorem 3.2.4 given here turns out to be surprisingly simple. First attempts in [93] (where $\operatorname{TCF}(T)$ is called $\operatorname{LIM}(T)$ ) have been considerably different, not as simple and only applicable to continuous TCFs.

Remark 3.2.10. In view of $\operatorname{TCF}(T)=\Psi(\Theta(T))$ (cf. Theorem 3.2.4 and (3.8) ) positive definiteness of elements of $\mathrm{TCF}(T)$ may also be derived from Theorem 2.3 .5 and Lemma A.1.3.

\subsection{Operations on TCFs}

Well-known operations on non-negative correlation functions include convex combinations, products and pointwise limits. Interestingly, the same operations are still admissable for $\operatorname{TCF}(T)$. Further operations on TCFs of stationary processes on $\mathbb{R}^{d}$ will be addressed in Chapter 4, Section 4.4.

Proposition 3.3.1. a) $\mathrm{TCF}(T)$ is convex.

b) $\operatorname{TCF}(T)$ is compact w.r.t. pointwise convergence.

c) $\operatorname{TCF}(T)$ is closed under pointwise multiplication.

Proof. Convexity of $\mathrm{TCF}(T)=\operatorname{MAX}(T)$ (cf. Theorem 3.2.4 follows from Corollary 2.4.1 and (3.8). Compactness of TCF $(T)$ follows from its closedness (cf. Theorem 3.2.4 , since $\operatorname{TCF}(T)$ is a subset of the compact space $[0,1]^{T \times T}$. Finally, let $\chi_{1}$ and $\chi_{2}$ be in $\operatorname{TCF}(T)=\operatorname{TCF}_{\infty}(T)$ with corresponding processes $X^{(1)}$ and $X^{(2)}$ with upper endpoint $x^{*}=\infty$. We choose the processes $X^{(1)}$ and $X^{(2)}$ to be independent. Then the process $X^{(3)}:=X^{(1)} \wedge X^{(2)}$ has upper endpoint $\infty$ and satisfies

$$
\mathbb{P}\left(X_{s}^{(3)}>x \mid X_{t}^{(3)}>x\right)=\mathbb{P}\left(X_{s}^{(1)}>x \mid X_{t}^{(1)}>x\right) \cdot \mathbb{P}\left(X_{s}^{(2)}>x \mid X_{t}^{(2)}>x\right) .
$$

Consequently, the TCF $\chi_{3}$ of $X^{(3)}$ is the product $\chi_{3}=\chi_{1} \cdot \chi_{2}$. 
Remark 3.3.2. If a set of functions is closed under convex combinations, products and pointwise limits, this allows for further operations built from these. For instance, we may apply convergent power series with positive and suitably normalized coefficients, e.g. if $\chi \in \operatorname{TCF}(T)$, then also $1-(1-\chi)^{q} \in \operatorname{TCF}(T)$ for $q \in(0,1)$.

Remark 3.3.3. In Proposition 3.3.1 we easily derived the product operation for $\operatorname{TCF}(T)$ and thus also for $\operatorname{MAX}(T)$. Using only the description of $\operatorname{MAX}(T)$ deriving the product operation is a subtle matter. In Section 3.4 we take advantage from the connection $\operatorname{TCF}(T)=\operatorname{MAX}(T)$ the other way around in order to derive that $\operatorname{TCF}(T)$ is bounded by finite-dimensional inequalities.

\subsection{Characterization by finite-dimensional inequalities}

In this section we show that the set $\operatorname{TCF}(T)$ is completely characterized by finitedimensional inequalities. This is not evident since elements of $\operatorname{TCF}(T)$ are defined by a limiting procedure. We benefit from the connection

$$
\operatorname{TCF}(T)=\operatorname{MAX}(T)=\Psi(\Theta(T))
$$

(cf. Theorem 3.2.4 and (3.8)) in the first place. For a finite set $M$ the set $\mathrm{TCF}(M)$ is in fact a convex polytope. Up to the fourvariate case we compute the vertices and bounding hyperplanes (i.e. defining inequalities) of $\operatorname{TCF}(M)$ explicitly. At least theoretically, there is an algorithm that provides vertices and inequalities of any order. As a byproduct, we may also formulate an algorithm that solves the inverse problem of finding a multivariate max-stable distribution realizing a prescribed TCF, even though we expect computations to be tedious and unfeasible already for lowdimensional cases.

Restrictions and extensions It follows from Theorem 2.3.5 that the set $\Theta(T)$ of ECFs of simple max-stable processes is completely characterized by finite-dimensional inequalities. In order to draw an analogy to TCFs later on, we formulate the following simple fact:

Corollary 3.4.1. Let $\theta: \mathcal{F}(T) \rightarrow \mathbb{R}$ be a function on the finite subsets of $T$. Then

$$
\left.\theta \in \Theta(T) \quad \Longleftrightarrow \quad \theta\right|_{\mathcal{F}(M)} \in \Theta(M) \quad \forall \emptyset \neq M \in \mathcal{F}(T)
$$

where $\left.\theta\right|_{\mathcal{F}(M)}$ denotes the restriction of $\theta$ to $\mathcal{F}(M)$.

For an arbitrary subset $S \subset T$, there are various ways to extend an ECF $\theta \in \Theta(S)$ to an ECF in $\Theta(T)$. Exemplarily we state the following two trivial extensions. 
Lemma 3.4.2. Let $S \subset T$ be a subset of $T$. If $\theta: \mathcal{F}(S) \rightarrow \mathbb{R}$ is an ECF in $\Theta(S)$, then the following two functions $\theta_{1}$ and $\theta_{2}$ extend $\theta$ to an $E C F$ in $\Theta(T)$ :

$$
\begin{array}{ll}
\theta_{1}: \mathcal{F}(T) \rightarrow \mathbb{R}, & \theta_{1}(A)=\theta(A \cap S)+\mathbb{1}_{A \cap(T \backslash S) \neq \emptyset}, \\
\theta_{2}: \mathcal{F}(T) \rightarrow \mathbb{R}, & \theta_{2}(A)=\theta(A \cap S)+|A \cap(T \backslash S)| .
\end{array}
$$

Proof. Let $X=\left\{X_{s}\right\}_{s \in S}$ be a simple max-stable process on $S$ with ECF $\theta$. Choose independently from $X$ i.i.d. standard Fréchet random variables $Y$ and $\left\{Y_{t}\right\}_{t \in T \backslash S}$. Then $\theta_{1}$ is the ECF of the simple max-stable process $Z_{t}=X_{t} \cdot \mathbb{1}_{t \in S}+Y \cdot \mathbb{1}_{t \in T \backslash S}$ and $\theta_{2}$ is the ECF of the simple max-stable process $Z_{t}=X_{t} \cdot \mathbb{1}_{t \in S}+Y_{t} \cdot \mathbb{1}_{t \in T \backslash S}$.

Analogously to Lemma 3.4 .2 , any $\chi \in \mathrm{TCF}(S)$ extends (trivially) to an element in $\operatorname{TCF}(T)$ if $S$ is a subset of $T$.

Lemma 3.4.3. Let $S \subset T$ be a subset of $T$. If $\chi: S \times S \rightarrow \mathbb{R}$ is a $T C F$ in $\operatorname{TCF}(S)$, then the following two functions $\chi_{1}$ and $\chi_{2}$ extend $\chi$ to a TCF in $\operatorname{TCF}(T)$ :

$$
\begin{array}{ll}
\chi_{1}: T \times T \rightarrow \mathbb{R}, & \chi_{1}(s, t)=\chi(s, t) \cdot \mathbb{1}_{\{s, t\} \subset S}+\mathbb{1}_{\{s, t\} \subset T \backslash S}, \\
\chi_{2}: T \times T \rightarrow \mathbb{R}, & \chi_{2}(s, t)=\chi(s, t) \cdot \mathbb{1}_{\{s, t\} \subset S}+\delta_{s t} \cdot \mathbb{1}_{\{s, t\} \subset T \backslash S} .
\end{array}
$$

It follows that the set $\operatorname{TCF}(T)$ is also fully characterized by finite-dimensional projections:

Proposition 3.4.4. Let $\chi: T \times T \rightarrow \mathbb{R}$. Then

$$
\left.\chi \in \operatorname{TCF}(T) \quad \Longleftrightarrow \quad \chi\right|_{M \times M} \in \operatorname{TCF}(M) \quad \forall \emptyset \neq M \in \mathcal{F}(T),
$$

where $\left.\chi\right|_{M \times M}$ denotes the restriction of $\chi$ to $M \times M$.

Proof. If $\chi \in \mathrm{TCF}(T)$, then necessarily $\left.\chi\right|_{S \times S} \in \mathrm{TCF}(S)$ for any subset $S \subset T$. To show the reverse implication, let $\chi: T \times T \rightarrow \mathbb{R}$ such that $\left.\chi\right|_{M \times M} \in \operatorname{TCF}(M)$ for all $\emptyset \neq M \in \mathcal{F}(T)$. We need to show that $\chi \in \operatorname{TCF}(T)$, which is equivalent to $\{\chi\} \cap \operatorname{TCF}(T) \neq \emptyset$. Further, we write $\operatorname{pr}_{M \times M}$ for the natural projection $\operatorname{pr}_{M \times M}$ : $[0, \infty)^{T \times T} \rightarrow[0, \infty)^{M \times M}$ for $\emptyset \neq M \in \mathcal{F}(T)$. Then $\chi \in \operatorname{TCF}(T)$ is equivalent to

$$
\emptyset \neq \bigcap_{\emptyset \neq M \in \mathcal{F}(T)} \operatorname{pr}_{M \times M}^{-1}\left(\left\{\left.\chi\right|_{M \times M}\right\}\right) \cap \operatorname{TCF}(T)=\bigcap_{\emptyset \neq M \in \mathcal{F}(T)} V(M),
$$

where

$$
V(M):=\operatorname{pr}_{M \times M}^{-1}\left(\left\{\left.\chi\right|_{M \times M}\right\}\right) \cap \operatorname{TCF}(T) .
$$


Since $\mathrm{TCF}(T)$ is compact (cf. Theorem 3.2.4 and Proposition 3.2.7) and $V(M) \subset$ $\operatorname{TCF}(T)$ are closed for any $\emptyset \neq M \in \mathcal{F}(T)$, it suffices to verify the finite intersection property for the system of sets $\{V(M)\}_{\emptyset \neq M \in \mathcal{F}(T)}$ in order to show that $\chi \in \operatorname{TCF}(T)$ (cf. e.g. [3, Theorem 2.31]). To this end, let $M_{1}, \ldots, M_{k}$ be non-empty finite subsets of $T$. By definition of $V(M)$, we have

$$
V\left(\bigcup_{i=1}^{k} M_{i}\right) \subset \bigcap_{i=1}^{k} V\left(M_{i}\right) .
$$

Therefore, it suffices to show that $V(M) \neq \emptyset$ for $\emptyset \neq M \in \mathcal{F}(T)$. Finally, this follows from the initial assumption $\left.\chi\right|_{M \times M} \in \mathrm{TCF}(M)$ together with Lemma 3.4.3. since both imply the existence of some $\tilde{\chi} \in \mathrm{TCF}(T)$ with $\left.\tilde{\chi}\right|_{M \times M}=\left.\chi\right|_{M \times M}$, which is again equivalent to $V(M) \neq \emptyset$. This finishes the proof.

\subsubsection{The convex polytopes $\Theta(M)$ and $\operatorname{TCF}(M)$}

Definition 3.4.5. A subset $P \subset \mathbb{R}^{n}$ is a convex polytope if $P$ is bounded and $P$ can be represented as $P=\left\{x \in \mathbb{R}^{n}: A x \leq a\right\}$ for an $m \times n$-matrix $A$ and an $m$-vector $a$ for some $m \in \mathbb{N}$ (where $\leq$ is meant componentwise). A vertex of $P$ is an extremal point of $P$ (cf. Definition A.4.1).

For a non-empty finite set $M$ the set of ECFs $\Theta(M) \subset \mathbb{R}^{\mathcal{F}(M)}$ is defined by $\theta(\emptyset)=0, \theta(\{t\})=1$ for $t \in M$ and the $2^{|M|}-1$ inequalities 2.12 stemming from complete alternation (cf. Theorem 2.3.5). By these properties, $\Theta(M)$ is automatically bounded by $|M|$ and thus, we may regard $\Theta(M)$ as a convex polytope in $\mathbb{R}^{n(M)}$, where $n(M)=2^{|M|}-|M|-1$. Since

$$
\operatorname{TCF}(M)=\Psi(\Theta(M))
$$

(cf. 3.10 and (3.7)), it follows that the set $\operatorname{TCF}(M) \subset[0,1]^{M \times M}$ is also a convex polytope. Moreover, $\chi \in \operatorname{TCF}(M)$ is symmetric and takes the value 1 on the diagonal. Therefore, we may regard $\operatorname{TCF}(M)$ as a convex polytope in $\mathbb{R}^{k(M)}$, where $k(M)=\left(\begin{array}{c}|M| \\ 2\end{array}\right)=\frac{|M|(|M|-1)}{2}$. So finally, Proposition 3.4.4 gives the following theorem.

Theorem 3.4.6. The set $\operatorname{TCF}(T) \subset[0,1]^{T \times T}$ can be defined by finite-dimensional inequalities.

Let us take a closer look at how to obtain these inequalities that define the set $\operatorname{TCF}(M)$ for a non-empty finite set $M$. At least theoretically, all of the defining inequalities (or equivalently bounding hyperplanes) and the vertices of the convex 
The set $\Theta(M)$ is a convex polytope in $\mathbb{R}^{\left(2^{|M|}-|M|-1\right)}$ with bounding

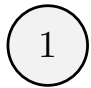
hyperplanes as given by the $2^{|M|}-1$ inequalities 2.12 where $\theta(\emptyset)=0$ and $\theta(\{t\})=1$ for $t \in T$ has to be taken into account. From these inequalities compute the vertices $\left\{U_{1}, \ldots, U_{a(M)}\right\}$ of the polytope $\Theta(M)$.
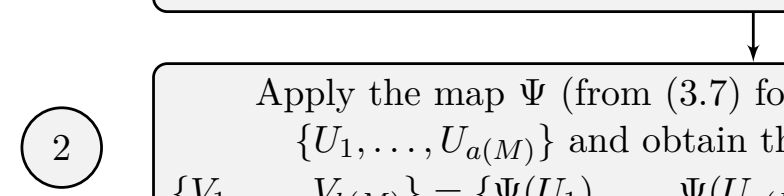

Apply the map $\Psi$ (from (3.7) for $T=M$ ) to these vertices

$\left\{U_{1}, \ldots, U_{a(M)}\right\}$ and obtain the set of potential vertices $\left\{V_{1}, \ldots, V_{b(M)}\right\}=\left\{\Psi\left(U_{1}\right), \ldots, \Psi\left(U_{a(M)}\right)\right\}$ of $\operatorname{TCF}(M) \subset \mathbb{R}^{|M|(|M|-1) / 2}$.

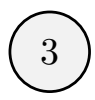

Remove all points from $\left\{V_{1}, \ldots, V_{b(M)}\right\}$ that are inner points of the convex hull of $\left\{V_{1}, \ldots, V_{b(M)}\right\}$ and thus, obtain the actual set of vertices $\left\{W_{1}, \ldots, W_{c(M)}\right\}$ of $\operatorname{TCF}(M)$.

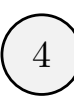

From these vertices $\left\{W_{1}, \ldots, W_{c(M)}\right\}$ compute the bounding hyperplanes of $\operatorname{TCF}(M)$ and thus, obtain the defining inequalities of $\operatorname{TCF}(M)$.

Algorithm 3.1: Ad-hoc strategy to compute the vertices and defining inequalities of the convex polytope $\operatorname{TCF}(M)$ from 2.12 .

polytope $\operatorname{TCF}(M)$ should be computable by Algorithm 3.1. which is evident from the above. Surely, this algorithm describes only the rough steps to be taken and is a rather naive approach. If one is only interested in the inequalities describing $\operatorname{TCF}(M)$ (and not the vertices), more elaborate approaches are summarized and presented in [46]. In fact, it seems adequate to use the Equality Set Projection method from [46] that has a linear complexity in the number of obtained inequalities. Since we are also interested in the vertices of $\operatorname{TCF}(M)$, we follow Algorithm 3.1 in the trivariate case and fourvariate case. Each step can be improved when using symmetries and an intelligent exclusion of cases. However, to us it seems a hard problem to formulate general "short-cuts" for arbitrary dimensions. The fourvariate case requires already a delicate distinction and exclusion of cases. Here we summarize the results.

The trivariate case For $M=\{1,2,3\}$, the convex polytope $\Theta(\{1,2,3\}) \subset \mathbb{R}^{4}$ is bounded by the $2^{3}-1=7$ hyperplanes that are given by

$$
\bigvee\left(\theta_{12}, \theta_{13}, \theta_{23}, \theta_{12}+\theta_{13}+\theta_{23}-3\right) \leq \theta_{123} \leq \bigwedge\left(\theta_{12}+\theta_{13}, \theta_{12}+\theta_{23}, \theta_{13}+\theta_{23}\right)-1
$$




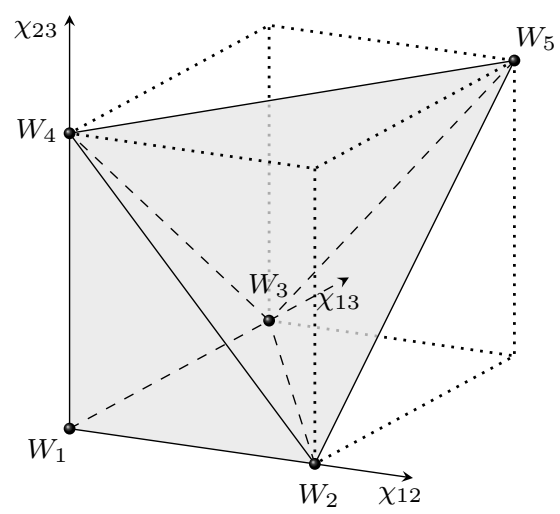

Figure 3.1.: The convex polytope $\operatorname{TCF}(\{1,2,3\})$ as a subset of the cube $[0,1]^{3}$ when we consider elements $\chi \in \operatorname{TCF}(\{1,2,3\})$ as being defined by the three values $\left(\chi_{12}, \chi_{13}, \chi_{23}\right)$. It has 5 vertices $\left\{W_{1}, \ldots, W_{5}\right\}$ and is clearly bounded by 6 hyperplanes, see also Table 3.1 .

(cf. (2.13) and [85, (13)]). Table 3.1 lists the vertices of $\Theta(\{1,2,3\}) \subset \mathbb{R}^{4}$ and the vertices of $\operatorname{TCF}(\{1,2,3\}) \subset \mathbb{R}^{3}$. Figure 3.1 illustrates the convex polytope $\operatorname{TCF}(\{1,2,3\})$. It can be easily seen that $\operatorname{TCF}(\{1,2,3\})$ is bounded by 6 hyperplanes that yield the following defining inequalities for $\operatorname{TCF}(\{1,2,3\})$ :

$$
\begin{array}{ll}
\chi_{12} \geq 0 & (3 \text { inequalities }) \\
\chi_{12}+\chi_{13}-\chi_{23} \leq 1 & (3 \text { triangle inequalities }) .
\end{array}
$$

The last three inequalities will be called triangle inequalities as they involve three points, where one of them takes a distinguished role. They are in fact triangle inequalities in the usual sense if we consider $\eta:=1-\chi$ instead of $\chi$.

The fourvariate case For $M=\{1,2,3,4\}$, the convex polytope $\Theta(\{1,2,3,4\}) \subset$ $\mathbb{R}^{11}$ is bounded by $2^{4}-1=15$ hyperplanes as given by the inequalities 2.12 . Table 3.2 lists the 42 vertices of $\Theta(\{1,2,3,4\}) \subset \mathbb{R}^{11}$. Following Algorithm 3.1, we obtain 15 actual vertices for $\operatorname{TCF}(\{1,2,3,4\}) \subset \mathbb{R}^{6}$ as listed in Table 3.3 and 22 bounding hyperplanes. They consist of the following three types and yield the 22 defining inequalities for $\operatorname{TCF}(\{1,2,3,4\})$ :

$$
\begin{array}{ll}
\chi_{12} \geq 0 & (6 \text { inequalities }) \\
\left(\chi_{12}+\chi_{13}+\chi_{14}\right)-\left(\chi_{23}+\chi_{24}+\chi_{34}\right) \leq 1 & (4 \text { tetrahedron inequalities }) \\
\chi_{12}+\chi_{13}-\chi_{23} \leq 1 & (12 \text { triangle inequalities })
\end{array}
$$

Compared to the trivariate case, we obtain one additional type of inequalities 3.13 . We call them tetrahedron inequalities as they involve four points, where one of them takes a distinguished role. 


\begin{tabular}{|c|c|c|c|c|c|c|c|c|c|}
\hline \multicolumn{5}{|c|}{ Vertices of $\Theta(\{1,2,3\})$} & \multicolumn{4}{|c|}{ Vertices of $\operatorname{TCF}(\{1,2,3\})$} & \multirow[t]{2}{*}{ Partitions of $\{1,2,3\}$} \\
\hline & $\theta_{12}$ & $\theta_{13}$ & $\theta_{23}$ & $\theta_{123}$ & & $\chi_{12}$ & $\chi_{13}$ & $\chi_{23}$ & \\
\hline$U_{1}$ & 2 & 2 & 2 & 3 & $W_{1}$ & 0 & 0 & 0 & $\{\{1\},\{2\},\{3\}\}$ \\
\hline$U_{2}$ & 1 & 2 & 2 & 2 & $W_{2}$ & 1 & 0 & 0 & $\{\{1,2\},\{3\}\}$ \\
\hline$U_{3}$ & 2 & 1 & 2 & 2 & $W_{3}$ & 0 & 1 & 0 & $\{\{1,3\},\{2\}\}$ \\
\hline$U_{4}$ & 2 & 2 & 1 & 2 & $W_{4}$ & 0 & 0 & 1 & $\{\{2,3\},\{1\}\}$ \\
\hline$U_{5}$ & 3 & 3 & 3 & 3 & & & & & \\
\hline$U_{6}$ & $\begin{array}{l}\overline{2} \\
1\end{array}$ & $\begin{array}{l}\overline{2} \\
1\end{array}$ & $\begin{array}{l}\overline{2} \\
1\end{array}$ & $\begin{array}{l}\overline{2} \\
1\end{array}$ & $W_{5}$ & 1 & 1 & 1 & $\{\{1,2,3\}\}$ \\
\hline
\end{tabular}

Table 3.1.: (trivariate case) The vertices $\left\{U_{1}, \ldots, U_{6}\right\}$ of the convex polytope $\Theta(\{1,2,3\}) \subset$ $\mathbb{R}^{4}$ and the corresponding vertices $\left\{W_{1}, \ldots, W_{5}\right\}$ of the convex polytope $\operatorname{TCF}(\{1,2,3\}) \subset \mathbb{R}^{3}$ are listed as obtained through the projecting map $\Psi$ (cf. Algorithm 3.1 for the notation). All vertices of $\operatorname{TCF}(\{1,2,3\})$ correspond to a partition of the set $\{1,2,3\}$, cf. Lemma 3.5.2

\begin{tabular}{|c|c|c|c|c|c|c|c|c|c|c|c|c|}
\hline \multicolumn{13}{|c|}{ Vertex types of $\Theta(\{1,2,3,4\})$} \\
\hline & & $\theta_{12}$ & $\theta_{13}$ & $\theta_{14}$ & $\theta_{23}$ & $\theta_{24}$ & $\theta_{34}$ & $\theta_{123}$ & $\theta_{124}$ & $\theta_{134}$ & $\theta_{234}$ & $\theta_{1234}$ \\
\hline $1 \times$ & $U_{1}$ & 2 & 2 & 2 & 2 & 2 & 2 & 3 & 3 & 3 & 3 & 4 \\
\hline $6 \times$ & $U_{2}$ & 1 & 2 & 2 & 2 & 2 & 2 & 2 & 2 & 3 & 3 & 3 \\
\hline & & 3 & 3 & & 3 & & & 3 & 5 & 5 & 5 & 5 \\
\hline $4 \times$ & $U_{8}$ & $\overline{2}$ & $\overline{2}$ & 2 & $\overline{2}$ & 2 & 2 & $\overline{2}$ & $\overline{2}$ & $\overline{2}$ & $\overline{2}$ & $\overline{2}$ \\
\hline $3 \times$ & $U_{12}$ & 1 & 2 & 2 & 2 & 2 & 1 & 2 & 2 & 2 & 2 & 2 \\
\hline $12 \times$ & $U_{15}$ & 2 & $\frac{3}{2}$ & $\frac{3}{2}$ & $\frac{3}{2}$ & $\frac{3}{2}$ & $\frac{3}{2}$ & 2 & 2 & $\frac{3}{2}$ & 2 & 2 \\
\hline $4 \times$ & $U_{27}$ & 1 & 1 & 2 & 1 & 2 & 2 & 1 & 2 & 2 & 2 & 2 \\
\hline $4 \times$ & $U_{31}$ & $\underline{4}$ & $\underline{4}$ & $\underline{5}$ & $\underline{4}$ & $\underline{5}$ & $\underline{5}$ & $\underline{5}$ & $\underline{5}$ & $\underline{5}$ & $\underline{5}$ & $\underline{5}$ \\
\hline & 31 & $\overline{3}$ & $\overline{3}$ & $\overline{3}$ & $\overline{3}$ & $\overline{3}$ & $\overline{3}$ & $\overline{3}$ & $\overline{3}$ & $\overline{3}$ & $\overline{3}$ & $\overline{3}$ \\
\hline $6 \times$ & $U_{35}$ & 1 & $\frac{3}{9}$ & $\underline{3}$ & $\underline{3}$ & $\underline{3}$ & $\underline{3}$ & $\underline{3}$ & $\underline{3}$ & $\underline{3}$ & $\underline{3}$ & $\underline{3}$ \\
\hline & & 4 & $\begin{array}{l}2 \\
4\end{array}$ & $\begin{array}{l}2 \\
4\end{array}$ & $\begin{array}{l}2 \\
4\end{array}$ & $\begin{array}{l}2 \\
4\end{array}$ & $\begin{array}{l}\overline{2} \\
4\end{array}$ & $\begin{array}{l}2 \\
4\end{array}$ & $\begin{array}{l}2 \\
4\end{array}$ & $\begin{array}{l}\overline{2} \\
4\end{array}$ & $\begin{array}{l}2 \\
4\end{array}$ & ${ }_{4}^{2}$ \\
\hline $1 \times$ & $U_{41}$ & $\overline{3}$ & $\overline{3}$ & $\overline{3}$ & $\overline{3}$ & $\overline{3}$ & $\overline{3}$ & $\overline{3}$ & $\overline{3}$ & $\overline{3}$ & $\frac{x}{3}$ & $\frac{4}{3}$ \\
\hline $1 \times$ & $U_{42}$ & 1 & 1 & 1 & 1 & 1 & 1 & 1 & 1 & 1 & 1 & 1 \\
\hline
\end{tabular}

Table 3.2.: (fourvariate case) The polytope $\Theta(\{1,2,3,4\}) \subset \mathbb{R}^{11}$ has 42 vertices, which can be devided into 10 types. For each type the table contains one representative and the total number of representatives due to symmetries. See also Algorithm 3.1 for the notation.

\begin{tabular}{ccccccccc}
\hline \multicolumn{8}{c}{ Vertex types of $\operatorname{TCF}(\{1,2,3,4\})$} & Partition types of $\{1,2,3,4\}$ \\
\hline $1 \times$ & $W_{1}$ & 0 & 0 & 0 & 0 & 0 & 0 & $\{\{1\},\{2\},\{3\},\{4\}\}$ \\
$6 \times$ & $W_{2}$ & 1 & 0 & 0 & 0 & 0 & 0 & $\{\{1,2\},\{3\},\{4\}\}$ \\
$3 \times$ & $W_{8}$ & 1 & 0 & 0 & 0 & 0 & 1 & $\{\{1,2\},\{3,4\}\}$ \\
$4 \times$ & $W_{11}$ & 1 & 1 & 0 & 1 & 0 & 0 & $\{\{1,2,3\},\{4\}\}$ \\
$1 \times$ & $W_{15}$ & 1 & 1 & 1 & 1 & 1 & 1 & $\{\{1,2,3,4\}\}$ \\
\hline
\end{tabular}

Table 3.3.: (fourvariate case) The polytope $\operatorname{TCF}(\{1,2,3,4\}) \subset \mathbb{R}^{6}$ has 15 vertices, which can be devided into 5 types. For each type the table contains one representative and the total number of representatives due to symmetries. All vertices of $\operatorname{TCF}(\{1,2,3,4\})$ correspond to a partition of the set $\{1,2,3,4\}$, cf. Lemma 3.5.2. 
Higher-order triangle inequalities from a conjecture of Matheron In higher-order cases, the problem size grows very fast, if we want to determine vertices and/or bounding hyperplanes of $\operatorname{TCF}(M)$ explicitly (cf. also Corollary 3.5.8 for a lower bound on the number of vertices). Still, we can derive certain "triangle inequalities of higher order" from a connection to Matheron's analysis [61] of variograms of processes with values in $\{0,1\}$. These inequalities are very likely to be among the hyperplanes that bound the convex polytope $\operatorname{TCF}(M)$.

Proposition 3.4.7. Let $\chi \in \mathrm{TCF}(T)$. Then for any finite subset $\emptyset \neq M \in \mathcal{F}(T)$ and any $e \in\{-1,0,1\}^{M}$ with $\sum_{t \in M} e_{t}=1$ we have that

$$
\sum_{s \in M, t \in M} e_{s} e_{t} \chi(s, t) \geq 1
$$

Proof. (cf. [61] and [24]) Let $\chi=\chi^{(X)} \in \mathrm{TCF}(T)$ and $X$ a corresponding realizing process such that $\chi(s, t)=\lim _{x \rightarrow \infty} \chi_{x}(s, t)$ with $\chi_{x}(s, t)=\mathbb{P}\left(X_{s}>x \mid X_{t}>x\right)$. We need to show 3.15 for any $\chi_{x}(x \in \mathbb{R})$. To this end, set $Y_{t}:=2 \mathbb{1}_{X_{t}>x}-1$ for $t \in T$, such that $Y_{t}$ takes values in $\{-1,1\}$ and $\sum_{t \in M} e_{t} Y_{t}$ is always odd. Therefore, $\mathbb{E}\left(\sum_{t \in M} e_{t} Y_{t}\right)^{2} \geq 1$, which entails

$$
\begin{aligned}
& 4 \mathbb{P}\left(X_{t}>x\right)\left[\sum_{s, t \in M} e_{s} e_{t} \chi_{x}(s, t)-1\right]=4 \mathbb{P}\left(X_{t}>x\right)\left[\sum_{s, t \in M} e_{s} e_{t}\left(\chi_{x}(s, t)-1\right)\right] \\
= & 4 \sum_{s, t \in M} e_{s} e_{t} \mathbb{E}\left(\mathbb{1}_{X_{s}>x} \mathbb{1}_{X_{t}>x}-\mathbb{1}_{X_{t}>x}\right)=\sum_{s, t \in M} e_{s} e_{t} \mathbb{E}\left(Y_{s} Y_{t}-1\right) \\
= & \sum_{s, t \in M} e_{s} e_{t} \mathbb{E} Y_{s} Y_{t}-1 \geq 0 .
\end{aligned}
$$

Remark 3.4.8. In 61] Matheron conjectures that the corresponding inequalities for $1-\chi$ are precisely the inequalities that describe the hyperplanes through the origin that bound the convex polytope of variograms of processes with values in $\{0,1\}$. Accordingly, we might suspect that the inequalities 3.15 describe the bounding hyperplanes of $\operatorname{TCF}(M)$ that have the point $(1,1, \ldots, 1)$ in common.

Example 3.4.9. The following example shows that the inequalities (3.15) give indeed new constraints for $\operatorname{TCF}(M)$ in addition to the fourvariate inequalities. We consider the symmetric matrix

$$
K=\left(\begin{array}{ccccc}
1 & 0.3 & 0.4 & 0.6 & 0.6 \\
0.3 & 1 & 0.3 & 0.6 & 0.6 \\
0.4 & 0.3 & 1 & 0.6 & 0.6 \\
0.6 & 0.6 & 0.6 & 1 & 0.4 \\
0.6 & 0.6 & 0.6 & 0.4 & 1
\end{array}\right)
$$


that contains only positive entries. The matrix $K$ satisfies all $\left(\begin{array}{l}5 \\ 3\end{array}\right) \cdot 3=30$ triangle inequalities 3.14 and all $\left(\begin{array}{l}5 \\ 4\end{array}\right) \cdot 4=20$ tetrahedron inequalities 3.13 . That means for any subset $M$ of $\{1,2,3,4,5\}$ with at most 4 elements the restriction $\left.K\right|_{M \times M}$ is an element of $\operatorname{TCF}(M)$. Moreover, the matrix $K$ is even positive definite. Nonetheless $K$ is not an element of $\operatorname{TCF}(\{1,2,3,4,5\})$ as it violates the "triangle inequality of higher order" 3.15 for $e=(1,1,1,-1,-1)$.

The inverse problem of a prescribed TCF Based on the previous considerations, we may also formulate algorithms (Algorithms 3.2 and 3.3 that solve the inverse problem of finding a multivariate distribution realizing a prescribed TCF. The problem has been addressed before in [28] and [30]. However the approach considered in [30] does not comprise dependent models whereas [28] does not comprise independence. Even though the methods described here cannot be computationally efficient, we want to emphasize that they cover all multivariate TCFs (and not a particular subclass). We obtain a whole polytope of solutions to the inverse problem if we consider all possible $\theta$ (and not only one) in the second step of Algorithm 3.2 .

Example 3.4.10. As a "toy example" we consider the trivariate case $M=\{1,2,3\}$ (which is indeed better understood involving (3.11), since the the preimage of a valid TCF $\chi$ under the map $\psi$ is only an interval with bounds given by (3.11)). Here the convex polytope $\operatorname{TCF}(\{1,2,3\})$ can be split into two tetrahedra

$$
\operatorname{TCF}(M)=\operatorname{conv}\left(W_{1}, W_{2}, W_{3}, W_{4}\right) \cup \operatorname{conv}\left(W_{5}, W_{2}, W_{3}, W_{4}\right)
$$

(cf. Figure 3.1). An element $\chi \in \operatorname{TCF}(\{1,2,3\})$ belongs to one or the other tetrahedron depending on whether $\chi_{12}+\chi_{13}+\chi_{23}$ is less or equal to 1 or greater or equal to 1. Therefore, Algorithm 3.3 can be abbreviated as follows: Let $\chi \in \operatorname{TCF}(\{1,2,3\})$. If $\chi_{12}+\chi_{13}+\chi_{23} \leq 1$, set

$$
A:=\left(\begin{array}{ccccccc}
1-\chi_{12}-\chi_{13} & 0 & 0 & \chi_{12} & \chi_{13} & 0 & 0 \\
0 & 1-\chi_{12}-\chi_{23} & 0 & \chi_{12} & 0 & \chi_{23} & 0 \\
0 & 0 & 1-\chi_{13}-\chi_{23} & 0 & \chi_{13} & \chi_{23} & 0
\end{array}\right) .
$$

Else set

$$
A:=\left(\begin{array}{ccccccc}
a_{1} & 0 & 0 & a_{3} & a_{2} & 0 & b \\
0 & a_{2} & 0 & a_{3} & 0 & a_{1} & b \\
0 & 0 & a_{3} & 0 & a_{2} & a_{1} & b
\end{array}\right) \quad \text { with } \quad\left(\begin{array}{c}
a_{1} \\
a_{2} \\
a_{3} \\
b
\end{array}\right)=\left(\begin{array}{cccc}
-1 & -1 & 1 & 1 \\
-1 & 1 & -1 & 1 \\
1 & -1 & -1 & 1 \\
1 & 1 & 1 & -1
\end{array}\right)\left(\begin{array}{c}
\chi_{12} \\
\chi_{13} \\
\chi_{23} \\
1
\end{array}\right) .
$$




(1) $\begin{aligned} & \text { Let } \chi \in \mathrm{TCF}(M) \subset[0,1]^{T \times T} \text { be given. Consider the } 2^{|M|}-1 \text { inequali- } \\ & \text { ties }(2.12) \text { for } \psi=\theta \text { and set } \theta(\emptyset)=0, \theta(\{t\})=1, \theta(s, t):=2-\chi(s, t) .\end{aligned}$
$\begin{aligned} & \text { Determine (at least one) } \theta \in \mathbb{R}^{\mathcal{F}(M)} \text { such that these inequalities } \\ & \text { are satisfied. (That means determine a point in the convex poly- } \\ & \text { tope } \Psi^{-1}(\{\chi\}) \cap \Theta(M) \text {, which is possible since } \chi \in \mathrm{TCF}(M) \text {.) }\end{aligned}$
(3) $\begin{gathered}\text { Simulate i.i.d. standard Fréchet random variables } \\ \left\{Z_{L}\right\}_{\emptyset \neq L \subset M} \text { and set } X_{t}=\bigvee_{t \in L \subset M} \tau_{L}^{M} Z_{L} \text { for } t \in M, \\ \left.\text { where }\left\{\tau_{L}^{M}\right\}_{\emptyset \neq L \subset M} \text { depend on } \theta \text { as in Theorem } 2.3 .5 \mathrm{~b}\right)\end{gathered}$

Algorithm 3.2: Ad-hoc strategy to simulate a random vector $X=\left\{X_{t}\right\}_{t \in M}$ realizing a prescribed $\chi \in \operatorname{TCF}(M)$. A preimage $\theta$ of $\chi$ under the map $\Psi$ (cf. (3.7) ) is determined and the corresponding distribution from Theorem 2.3 .5 is simulated.

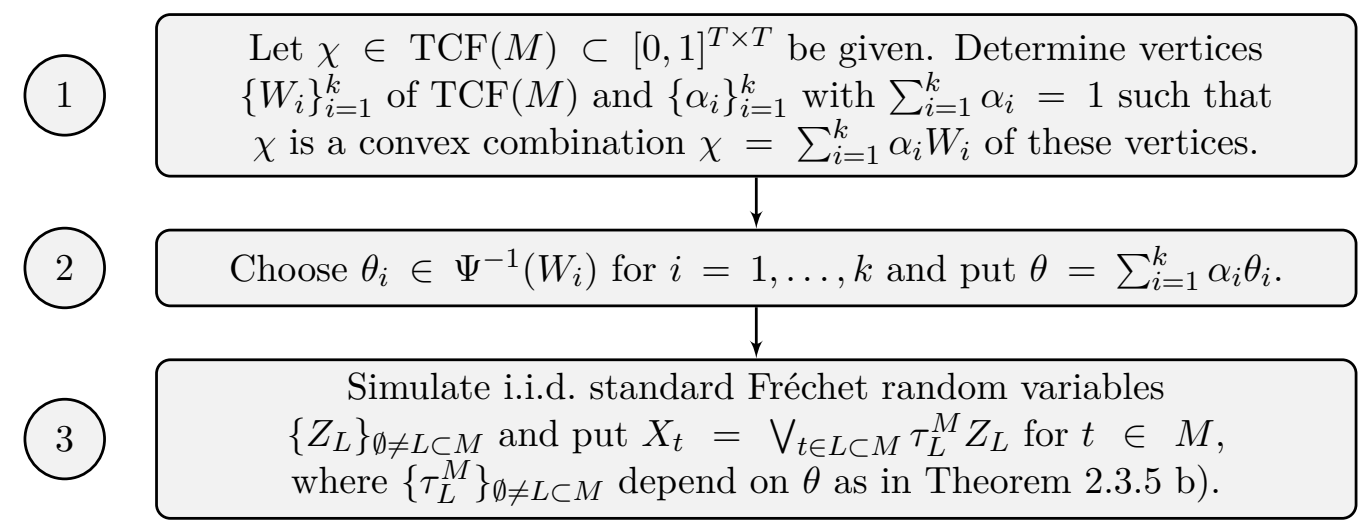

Algorithm 3.3: If the vertices of $\Theta(M)$ and $\operatorname{TCF}(M)$ are already known, this approach can be used to simulate a random vector $X=\left\{X_{t}\right\}_{t \in M}$ realizing a prescribed $\chi \in \mathrm{TCF}(M)$. As before in Algorithm 3.2 a preimage $\theta$ of $\chi$ under the map $\Psi$ (cf. (3.7) ) is determined and the corresponding distribution from Theorem 2.3.5 is simulated.

Simulate a random vector $Z=\left(Z_{1}, Z_{2}, Z_{3}, Z_{12}, Z_{13}, Z_{23}, Z_{123}\right)$ with i.i.d. standard Fréchet entries and obtain $X=\left(X_{1}, X_{2}, X_{3}\right)$ by $X_{i}=\bigvee_{\emptyset \neq L \subset\{1,2,3\}} A_{i, L} Z_{L}$. The random vector $X$ is simple max-stable and realizes $\chi$ as its TCF.

\subsection{Extremal TCFs}

Taking a closer look at the set of vertices of $\operatorname{TCF}(M)$ up to the fourvariate case, we note that in these cases the vertices of $\operatorname{TCF}(M)$ are in a 1-1 correspondence with partitions of the respective set $M$. 


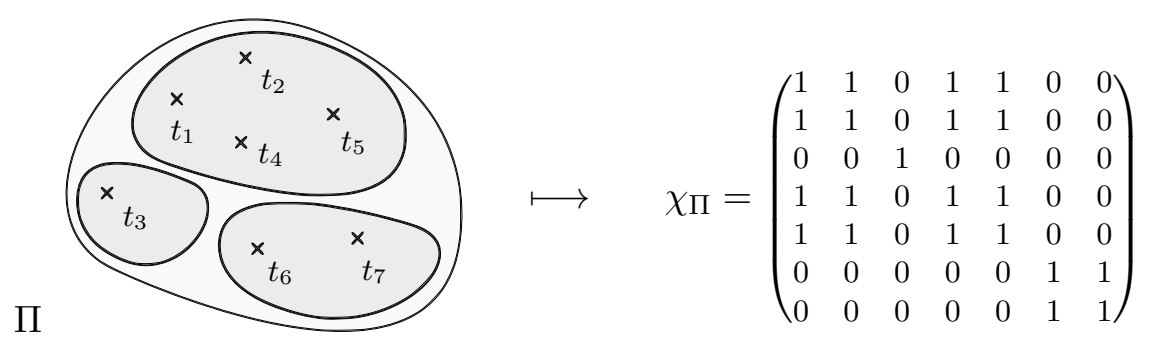

Figure 3.2.: Let $M=\left\{t_{1}, t_{2}, \ldots, t_{7}\right\}$. The partition $\Pi=\left\{\left\{t_{1}, t_{2}, t_{4}, t_{5}\right\},\left\{t_{3}\right\},\left\{t_{6}, t_{7}\right\}\right\}$ yields the TCF $\chi_{\Pi}(s, t)=\mathbb{1}_{\{s, t\} \subset\left\{t_{1}, t_{2}, t_{4}, t_{5}\right\}}+\mathbb{1}_{\{s, t\} \subset\left\{t_{3}\right\}}+\mathbb{1}_{\{s, t\} \subset\left\{t_{6}, t_{7}\right\}}$ which is represented in matrix form here, see Lemma 3.5.2.

Definition 3.5.1. Let $T$ be a non-empty set. Then a subset $\Pi$ of the powerset $\mathcal{P}(T)$ of $T$ is a partition of $T$, if it consists of disjoint non-empty subsets of $T$, such that $\bigcup_{A \in \Pi} A=T$ holds. The set of all partitions of $T$ will be denoted by $\Pi(T)$.

Now, any partition of $T$ defines an extremal point of $\operatorname{TCF}(T)$ as follows, see also Figure 3.2 for an example.

Lemma 3.5.2. Let $T$ be a non-empty set. There is an injective map from $\mathbf{\Pi}(T)$ to $\mathrm{TCF}(T)$ given by

$$
\Pi \longmapsto \chi_{\Pi} \quad \text { with } \quad \chi_{\Pi}(s, t)=\sum_{A \in \Pi} \mathbb{1}_{\{s, t\} \subset A}
$$

Proof. Let $\left\{X_{A}\right\}_{A \in \Pi}$ be i.i.d. standard Fréchet random variables. For $t \in T$ set $X_{t}:=X_{A}$ if $t \in A$ for $A \in \Pi$. Then $X=\left\{X_{t}\right\}_{t \in T}$ is a stochastic process with TCF $\chi_{\Pi}$. Apparently, different partitions lead to different TCFs.

Proposition 3.5.3. Any $\chi_{\Pi} \in \mathrm{TCF}(T)$ stemming from a partition $\Pi \in \Pi(T)$ is an extremal point of $\operatorname{TCF}(T)$

$$
\left\{\chi_{\Pi}: \Pi \in \Pi(T)\right\} \subset \operatorname{ex}(\operatorname{TCF}(T))
$$

The closure of the convex hull of these elements is a subset of $\mathrm{TCF}(T)$

$$
\overline{\operatorname{conv}}\left(\left\{\chi_{\Pi}: \Pi \in \Pi(T)\right\}\right) \subset \operatorname{TCF}(T) .
$$

Proof. Those elements $\chi_{\Pi} \in \mathrm{TCF}(T)$ stemming from a partition $\Pi \in \Pi(T)$ take values in $\{0,1\}$ only (the extremal points of $[0,1]$ ). Therefore, they cannot be decomposed non-trivially as a convex combination of TCFs. The second statement follows, since $\operatorname{TCF}(T)$ is convex and closed (cf. Proposition 3.3.1). 
Remark 3.5.4. Equality in 3.16) would immediately imply equality in (3.17) due to the Krein-Milman-Theorem (cf. Theorem A.4.2).

Up to the fourvariate case, equality in (3.16) is indeed established (cf. Tables 3.1 and 3.3 .

$\operatorname{ex}(\operatorname{TCF}(M))=\left\{\chi_{\Pi}: \Pi \in \Pi(M)\right\} \quad$ in case $\quad M=\{1\},\{1,2\},\{1,2,3\},\{1,2,3,4\}$.

Let us consider two more observations (Lemma 3.5.5 and Observation 3.5.7.) that might be helpful to understand the general case.

Lemma 3.5.5. a) Let $M$ be a non-empty finite set. Then $\chi \in \operatorname{TCF}(M)$ if and only if $\chi$ can be represented as

$$
\chi(s, t)=\sum_{\emptyset \neq L \subset M} \tau_{L} \mathbb{1}_{\{s, t\} \subset L}
$$

for non-negative coefficients $\left\{\tau_{L}\right\}_{\emptyset \neq L \subset M}$ that satisfy

$$
\sum_{\emptyset \neq L \subset M} \tau_{L} \mathbb{1}_{t \in L}=1 \quad \forall t \in M
$$

b) Let $T$ be an arbitrary non-empty set. Then $\chi \in \mathrm{TCF}(T)$ if and only if $\chi$ can be represented as

$$
\chi(s, t)=\int_{\mathcal{P}(T) \backslash\{\emptyset\}} \mathbb{1}_{\{s, t\} \subset Q} \mu(\mathrm{d} Q)
$$

for a positive Radon measure $\mu$ on $\mathcal{P}(T) \backslash\{\emptyset\}$ with

$$
\int_{\mathcal{P}(T) \backslash\{\emptyset\}} \mathbb{1}_{t \in Q} \mu(\mathrm{d} Q)=1 \quad \forall t \in M .
$$

Proof. Part b) follows from $\operatorname{TCF}(T)=\operatorname{MAX}(T)=\Psi(\Theta(T))$ (cf. Theorem 3.2.4 and (3.8) and Corollary 2.4.4 and entails part a), where $\mu$ decomposes into point masses $\mu(\{L\})=: \tau_{L}($ cf. Remark 2.4.5).

Remark 3.5.6. Both representations in Lemma 3.5.5 are not (!) uniquely determined by the TCF $\chi$.

Observation 3.5.7. Assume non-negative coefficients $\alpha_{\Pi}$ are given for $\Pi \in \Pi(M)$ such that $\sum_{\Pi \in \Pi(M)} \alpha_{\Pi}=1$. Or equivalently (via $\mathbb{P}(\Pi)=\alpha_{\Pi}$ ) let $\mathbb{P}$ be a probability measure on $\Pi(M)$. Set

$$
\tau_{L}:=\sum_{\Pi \in \Pi(M): L \in \Pi} \alpha_{\Pi}=\mathbb{P}(L \in \Pi)
$$


for $\emptyset \neq L \subset M$. Then 3.19 is automatically satisfied, since

$$
\sum_{\emptyset \neq L \subset M} \tau_{L} \mathbb{1}_{t \in L}=\sum_{\emptyset \neq L \subset M: t \in L} \mathbb{P}(L \in \Pi)=\mathbb{P}(\exists L \in \Pi: t \in L)=1 \quad \forall t \in L .
$$

The TCFs defined by $\left\{\tau_{L}\right\}_{\emptyset \neq L \subset M}$ (cf. (3.18)) and by $\left\{\alpha_{\Pi}\right\}_{\Pi \in \Pi(M)}$ (cf. Lemma 3.5.2 coincide by the choice of 3.20

$$
\sum_{\emptyset \neq L \subset M} \tau_{L} \mathbb{1}_{\{s, t\} \subset L}=\sum_{\Pi \in \Pi(M)} \alpha_{\Pi} \chi_{\Pi}(s, t) \quad \forall(s, t) \in M \times M .
$$

Naturally, the question arises whether some converse statement can be made in order to establish the coincidence of $\left\{\chi_{\Pi}: \Pi \in \Pi(M)\right\}$ and $\operatorname{ex}(\operatorname{TCF}(M))$. However, beware that it is possible to choose non-negative coefficients $\left\{\tau_{L}\right\}_{\emptyset \neq L \subset M}$ satisfying the condition (3.19), such that no probability measure $\mathbb{P}$ on $\Pi(M)$ satisfies 3.20 for all $\emptyset \neq L \subset M$. This is possible even in the trivariate case $M=\{1,2,3\}$. For instance, set $\tau_{1}=\tau_{2}=\tau_{3}=0.1, \tau_{12}=\tau_{13}=\tau_{23}=0.2, \tau_{123}=0.5$, which fulfills (3.19) since $0.1+0.2+0.2+0.5=1$, and assume the existence of $\mathbb{P}$ with 3.20 . Then

$$
\begin{aligned}
0.1 & =\mathbb{P}(\{1\} \in \Pi)=\mathbb{P}(\Pi=\{\{1\},\{2\},\{3\}\})+\mathbb{P}(\Pi=\{\{1\},\{2,3\}\}) \\
& =\mathbb{P}(\Pi=\{\{1\},\{2\},\{3\}\})+\mathbb{P}(\{2,3\} \in \Pi)=\mathbb{P}(\Pi=\{\{1\},\{2\},\{3\}\})+0.2
\end{aligned}
$$

yields a contradiction. Nonetheless, $\operatorname{ex}(\operatorname{TCF}(M))=\left\{\chi_{\Pi}: \Pi \in \Pi(M)\right\}$ is true in the trivariate case. Thus, better ideas to involve the non-uniqueness of coefficients $\tau_{L}$ in the representation 3.18 are needed.

Even though the general case remains an open question to us, it is appealing to believe that those TCFs stemming from partitions are precisely the extremal TCFs, since they allow for a convenient interpretation. The value $\chi(s, t)$ of a TCF $\chi$ would simply be the probability of $s$ and $t$ being equivalent under a random equivalence relation.

Inclusion (3.16) is useful (also without equality) in that it offers a new method to generate valid TCFs as a convex combination $\sum_{\Pi \in \Pi} \alpha_{\Pi} \chi_{\Pi}$ of partition TCFs $\chi_{\Pi}$ together with a corresponding simple max-stable distribution $\bigvee_{\Pi \in \Pi} \alpha_{\Pi} X_{\Pi}$, where $X_{\Pi}$ is the process described in the proof of Lemma 3.5.2. This also opens up a bridge to involve models for random partitions. One can be sure to cover a fairly rich class of TCFs this way (all TCFs up to the fourvariate case). Moreover, we obtain a lower bound on the number of vertices of $\operatorname{TCF}(M)$ since the number of partitions of a set with $n$ elements is known to be the $n$th Bell number $B_{n}$ [1]. The first Bell numbers are (starting with $\left.B_{0}\right) 1,1,2,5,15,52,203,877,4140,21147,115975$. 
Corollary 3.5.8. The number of vertices of $\operatorname{TCF}(M)$ is greater or equal to the $|M|$ th Bell number $B_{|M|}$.

We also might try to verify the idea presented in Remark 3.4 .8 from the knowlege of (3.16), and vice versa, Propostition 3.4.7 might help to exclude further potential vertices of $\operatorname{TCF}(M)$ close to $(1,1, \ldots, 1)$. Finally, we want to remark that even if equality in (3.16) turns out to be true, the problem of identifying the bounding hyperplanes of $\operatorname{TCF}(M)$ will not be an easy one. We refer to [2] for a systematic study of hyperplanes spanned by vertices of the hypercube (and its limitations).

\subsection{TCFs compared to non-negative correlation functions}

Non-negative correlation functions A function $\varphi: T \times T \rightarrow \mathbb{R}$ is called positive definite if it is symmetric and for all $n \geq 1,\left\{t_{1}, \ldots, t_{n}\right\} \subset T$ and $\left\{a_{1}, \ldots, a_{n}\right\} \subset \mathbb{R}$

$$
\sum_{j, k=1}^{n} a_{j} a_{k} \varphi\left(t_{j}, t_{k}\right) \geq 0 .
$$

If $\varphi$ is additionally normalized to the value 1 on the diagonal, the function $\varphi$ is a correlation function. Denoting the set of non-negative correlation functions by

$$
\mathrm{CF}_{+}(T):=\left\{\varphi: T \times T \rightarrow[0, \infty): \begin{array}{l}
\varphi \text { is positive definite, } \\
\varphi(t, t)=1 \text { for all } t \in T .
\end{array}\right\},
$$

we have already seen that

$$
\mathrm{TCF}(T) \subset \mathrm{CF}_{+}(T)
$$

(cf. (3.1)). If $T$ contains only one or two elements, these sets coincide: $\operatorname{TCF}(\{t\})=$ $\mathrm{CF}_{+}(\{t\})=\{1\}$ and

$$
\operatorname{TCF}(\{s, t\})=\mathrm{CF}_{+}(\{s, t\})=\left\{\left(\begin{array}{ll}
1 & \rho \\
\rho & 1
\end{array}\right): \rho \in[0,1]\right\} .
$$

Otherwise for $|T| \geq 3$ the inclusion (3.21) is always proper regardless of $T$ being finite or infinite, as we shall see later on (cf. (3.22)).

Both classes, $\mathrm{TCF}(T)$ and $\mathrm{CF}_{+}(T)$, allow for convex combinations, products and pointwise limits (see Proposition 3.3.1 and e.g. [5, 3.1.11 and 3.1.12] for the statements for $\mathrm{CF}_{+}(T)$ ). In Chapter 4 , where we consider stationary processes on $\mathbb{R}^{d}$, we 
compare the turning bands operator and the validity of certain well-known parametric models such as the Whittle-Matérn model (cf. Section 4.4 and Table 4.4). Here, we focus on the finite-dimensional inequalities that characterize both classes.

\subsubsection{Finite-dimensional inequalities}

By definition, the set of functions $\mathrm{CF}_{+}(T)$ is characterized by finite inequalities. In analogy to Proposition 3.4 .4 for $\operatorname{TCF}(T)$ we formulate the following lemma.

Lemma 3.6.1. Let $\varphi: T \times T \rightarrow \mathbb{R}$. Then

$$
\left.\varphi \in \mathrm{CF}_{+}(T) \quad \Longleftrightarrow \quad \varphi\right|_{M \times M} \in \mathrm{CF}_{+}(M) \quad \forall \emptyset \neq M \in \mathcal{F}(T),
$$

where $\left.\varphi\right|_{M \times M}$ denotes the restriction of $\varphi$ to $M \times M$.

As for $\operatorname{TCF}(M)$, we may regard elements of $\mathrm{CF}_{+}(M)$ as elements of $\mathbb{R}^{k(M)}$, where $k(M)=\left(\begin{array}{c}|M| \\ 2\end{array}\right)=\frac{|M|(|M|-1)}{2}$. However, unlike $\operatorname{TCF}(M)$, the compact convex set $\mathrm{CF}_{+}(M)$ is not a polytope in $\mathbb{R}^{k(M)}$ if $M$ comprises at least three elements, but is merely bounded by the algebraic conditions of Sylvester's criterion, which says that a symmetric real matrix is positive definite if all its principal minors are nonnegative.

Example 3.6.2. In the trivariate case the set $\mathrm{CF}_{+}(M)$ is given by

$$
\mathrm{CF}_{+}(\{1,2,3\})=\left\{\left(\begin{array}{lll}
1 & x & y \\
x & 1 & z \\
y & z & 1
\end{array}\right): \begin{array}{l}
x, y, z \in[0,1] \\
1+2 x y z-\left(x^{2}+y^{2}+z^{2}\right) \geq 0
\end{array}\right\} .
$$

We plot $\mathrm{CF}_{+}(\{1,2,3\})$ in Figure 3.3 to illustrate how the polytope $\operatorname{TCF}(\{1,2,3\})$ is contained in $\mathrm{CF}_{+}(\{1,2,3\})$. Choosing $(x, y, z)=(0.6,0.6,0)$ for example, gives an element from $\mathrm{CF}_{+}(\{1,2,3\}) \backslash \mathrm{TCF}(\{1,2,3\})$, which is positive definite, but violates the triangle inequality 3.14.

Moreover, the same (trivial) extensions as in Lemma 3.4 .3 for $\operatorname{TCF}(T)$ also hold for $\mathrm{CF}_{+}(T)$.

Lemma 3.6.3. Let $S \subset T$ be a subset of $T$. If $\varphi: S \times S \rightarrow \mathbb{R}$ is a correlation function in $\mathrm{CF}_{+}(S)$, then the following two functions $\varphi_{1}$ and $\varphi_{2}$ extend $\varphi$ to an element of $\mathrm{CF}_{+}(T)$ :

$$
\begin{array}{ll}
\varphi_{1}: T \times T \rightarrow \mathbb{R}, & \varphi_{1}(s, t)=\varphi(s, t) \cdot \mathbb{1}_{\{s, t\} \subset S}+\mathbb{1}_{\{s, t\} \subset T \backslash S}, \\
\varphi_{2}: T \times T \rightarrow \mathbb{R}, & \varphi_{2}(s, t)=\varphi(s, t) \cdot \mathbb{1}_{\{s, t\} \subset S}+\delta_{s t} \cdot \mathbb{1}_{\{s, t\} \subset T \backslash S} .
\end{array}
$$



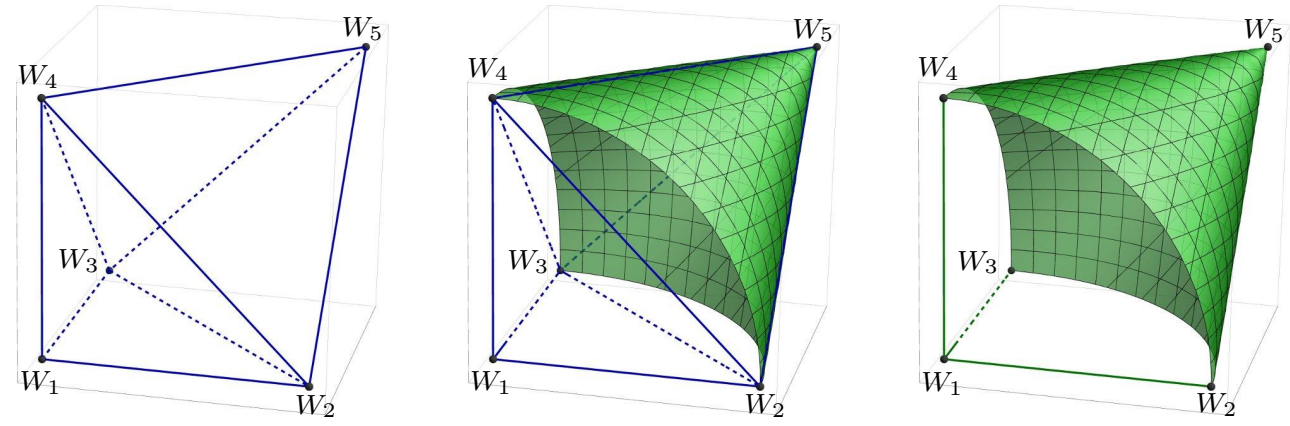

Figure 3.3.: The convex set $\mathrm{CF}_{+}(\{1,2,3\})$ (on the right) can be viewed as a subset of the cube $[0,1]^{3}$ when we consider elements as defined by the variables $(x, y, z)$ from Example 3.6.2 It is shown how $\operatorname{TCF}(\{1,2,3\})$ (on the left) is contained in $\mathrm{CF}_{+}(\{1,2,3\})$ (in the middle), cf. also Figure 3.1

Proof. Let $U=\left\{U_{s}\right\}_{s \in S}$ be a Gaussian process on $S$ where each one-dimensional marginal $U_{s}$ follows a standard normal distribution, such that $\varphi(s, t)=\mathbb{E} U_{s} U_{t}$ ist the covariance function of $U$. Choose independently from $U$ i.i.d. standard normally distributed random variables $V$ and $\left\{V_{t}\right\}_{t \in T \backslash S}$. Then $\varphi_{1}$ is the covariance function of the Gaussian process $W_{t}=U_{t} \cdot \mathbb{1}_{t \in S}+V \cdot \mathbb{1}_{t \in T \backslash S}$ and $\varphi_{2}$ is the covariance function of the Gaussian process $W_{t}=U_{t} \cdot \mathbb{1}_{t \in S}+V_{t} \cdot \mathbb{1}_{t \in T \backslash S}$.

Now, it follows from Example 3.6 .2 and Lemma 3.6 .3 that if $T$ comprises at least three elements $\{1,2,3\}$, the function

$$
\varphi: T \times T \rightarrow \mathbb{R}, \quad \varphi(s, t)=0.6 \cdot \mathbb{1}_{(s, t) \in\{(1,2),(2,1),(1,3),(3,1)\}}+\delta_{s t}
$$

belongs to $\mathrm{CF}_{+}(T)$, but cannot belong to $\mathrm{TCF}(T)$ because it violates the triangle inequality (3.14).

The fourvariate case We have already seen that the polytope $\operatorname{TCF}(\{1,2,3,4\})$ is bounded by 22 hyperplanes in $\mathbb{R}^{6}$. Apart from requiring elements to be non-negative, the non-trivial constraints for being an element of $\operatorname{TCF}(\{1,2,3,4\})$ consist of two types of inequalities, the triangle inequalities (3.14) and the tetrahedron inequalities (3.13). We compare these two types of inequalities with positive definiteness in Table 3.4 in the presence of non-negativity. For each constellation of fulfillment $(\boldsymbol{})$ or violation $(\mathbf{X})$ of these properties we provide an example whenever possible. Those elements in the table which are positive definite clearly belong to $\mathrm{CF}_{+}(\{1,2,3,4\})$. Since we know already that $\operatorname{TCF}(M) \subset \mathrm{CF}_{+}(M)$, it is obvious that there cannot be an example in the second row (triangle and tetrahedron inequalities fulfilled and positive definiteness not). In fact, the triangle inequalities alone suffice already 


\begin{tabular}{|c|c|c|c|}
\hline $\begin{array}{c}\text { triangle } \\
\text { inequalities }\end{array}$ & $\begin{array}{l}\text { tetrahedron } \\
\text { inequalities }\end{array}$ & $\begin{array}{c}\text { positive } \\
\text { definiteness }\end{array}$ & $\begin{array}{c}\text { example } \\
\left(\varphi_{12}, \varphi_{13}, \varphi_{14}, \varphi_{23}, \varphi_{24}, \varphi_{34}\right)\end{array}$ \\
\hline$\checkmark$ & $\checkmark$ & $\checkmark$ & $(0.5,0.5,0.5,0.5,0.5,0.5)$ \\
\hline$\checkmark$ & $\checkmark$ & $x$ & - \\
\hline$\checkmark$ & $x$ & $\checkmark$ & $(0.5,0.5,0.5,0.1,0.1,0.1)$ \\
\hline$\checkmark$ & $x$ & $x$ & - \\
\hline$x$ & $\checkmark$ & $\checkmark$ & $(0.8,0.8,0.8,0.5,0.5,0.5)$ \\
\hline$x$ & $\checkmark$ & $x$ & $(0.805,0.805,0.805,0.427,0.427,0.427)$ \\
\hline$\times$ & $x$ & $\checkmark$ & $(0.6,0.6,0.6,0.1,0.1,0.1)$ \\
\hline$\times$ & $x$ & $x$ & $(1.0,1.0,1.0,0.0,0.0,0.0)$ \\
\hline
\end{tabular}

Table 3.4.: The triangle inequalities 3.14$)$ and the tetrahedron inequalities $\sqrt{3.13}$ ) describe the non-trivial bounding hyperplanes of the convex polytope $\operatorname{TCF}(\{1,2,3,4\})$ that is contained in the set of positive definite functions $\mathrm{CF}_{+}(\{1,2,3,4\})$. For each constellation of fulfillment $(\boldsymbol{V})$ or violation $(\boldsymbol{X})$ of these properties we provide an example of a symmetric $4 \times 4$ matrix with 1 on the diagonal whenever this is possible for non-negative values.

to imply positive definiteness in the fourvariate case (see Proposition 3.6.5 below). In other words, the tetrahedron inequalities are not necessary to enforce positive definiteness. Therefore it is also not possible to provide an example in the fourth row (triangle inequalities fulfilled, but tetrahedron inequalities and positive definiteness not).

Let us denote the set of normalized non-negative symmetric functions that satisfy the triangle inequality by

$$
\operatorname{TRI}(T):=\left\{\begin{array}{ll} 
& \varphi(s, t)=\varphi(t, s) \\
\varphi: T \times T \rightarrow[0, \infty): & \varphi(s, t)+\varphi(t, r)-\varphi(s, r) \leq 1 \\
& \text { and } \varphi(t, t)=1 \text { for all } s, t, r \in T .
\end{array}\right\}
$$

Note that elements of $\operatorname{TRI}(T)$ are automatically bounded by 1 . Using this, it is easy to check that we have (trivial) extensions also for $\operatorname{TRI}(T)$ (as before for $\operatorname{TCF}(T)$ or $\left.\mathrm{CF}_{+}(T)\right)$.

Lemma 3.6.4. Let $S \subset T$ be a subset of $T$. If $\varphi: S \times S \rightarrow \mathbb{R}$ is an element of $\operatorname{TRI}(S)$, then the following two functions $\varphi_{1}$ and $\varphi_{2}$ extend $\varphi$ to an element of $\operatorname{TRI}(T)$ :

$$
\begin{array}{ll}
\varphi_{1}: T \times T \rightarrow \mathbb{R}, & \varphi_{1}(s, t)=\varphi(s, t) \cdot \mathbb{1}_{\{s, t\} \subset S}+\mathbb{1}_{\{s, t\} \subset T \backslash S}, \\
\varphi_{2}: T \times T \rightarrow \mathbb{R}, & \varphi_{2}(s, t)=\varphi(s, t) \cdot \mathbb{1}_{\{s, t\} \subset S}+\delta_{s t} \cdot \mathbb{1}_{\{s, t\} \subset T \backslash S} .
\end{array}
$$

Proposition 3.6.5. $\mathrm{TRI}(M) \subset \mathrm{CF}_{+}(M)$ if and only if $|M| \leq 4$.

Proof. Let $|M| \leq 4$. It suffices to consider the fourvariate case $M=\{1,2,3,4\}$. 
By definition, the set $\operatorname{TRI}(\{1,2,3,4\})$ is a convex polytope in $\mathbb{R}^{6}$. Computing the vertices of this polytope we find that $\operatorname{TRI}(\{1,2,3,4\})$ has 19 vertices: 15 of these vertices are precisely the vertices of $\operatorname{TCF}(\{1,2,3,4\})$ as listed in Table 3.3 and therefore also elements of $\mathrm{CF}_{+}(\{1,2,3,4\})$. The other 4 vertices are up to symmetries represented by the positive definite matrix

$$
\left(\begin{array}{cccc}
1 & 0.5 & 0.5 & 0.5 \\
0.5 & 1 & 0 & 0 \\
0.5 & 0 & 1 & 0 \\
0.5 & 0 & 0 & 1
\end{array}\right)
$$

Therefore, all vertices of the convex polytope $\operatorname{TRI}(\{1,2,3,4\})$ are contained in the convex set $\mathrm{CF}_{+}(\{1,2,3,4\})$, which entails $\operatorname{TRI}(\{1,2,3,4\}) \subset \mathrm{CF}_{+}(\{1,2,3,4\})$.

Let $|M| \geq 5$. In view of Lemma 3.6 .4 it suffices to consider the fivevariate case. We consider the following matrix

$$
K=\left(\begin{array}{ccccc}
1 & 0.3 & 0.3 & 0.6 & 0.6 \\
0.3 & 1 & 0.3 & 0.6 & 0.6 \\
0.3 & 0.3 & 1 & 0.6 & 0.6 \\
0.6 & 0.6 & 0.6 & 1 & 0.3 \\
0.6 & 0.6 & 0.6 & 0.3 & 1
\end{array}\right) .
$$

Although all triangle inequalities (3.14) are satisfied for $K$, the matrix $K$ is not positive definite. This yields a counterexample in $\operatorname{TRI}(M) \cap\left(\mathrm{CF}_{+}(M)\right)^{c}$ for all sets $M$ with at least five elements.

Remark 3.6.6. Instead of $\operatorname{TRI}(M) \subset \mathrm{CF}_{+}(M)$ which is wrong for $|M|>4$, it seems likely that

$$
\bigcap_{k=1}^{\left\lfloor\frac{|M|-1}{2}\right\rfloor} \operatorname{TRI}^{2 k+1}(M) \subset \mathrm{CF}_{+}(M)
$$

if $\operatorname{TRI}^{2 k+1}(M)$ denotes the symmetric [0,1]-valued functions on $T \times T$ that satisfy 3.15 for any $e \in\{-1,0,1\}^{M}$ with $\sum_{t \in M} e_{t}=1$ and $\sum_{t \in M}\left|e_{t}\right|=2 k+1$. This would be in accordance with Remark 3.4.8, 


\section{Max-stable processes sharing the same tail correlation function}

This chapter is based on joint work with Felix Ballani. In the interest of a better readability we enclose the proofs in a separate section at the end of the chapter.

\subsection{Stationary max-stable processes and their TCFs}

The tail correlation function (TCF) of a stationary max-stable process $X=\left\{X_{t}\right\}_{t \in \mathbb{R}^{d}}$ on $\mathbb{R}^{d}$ is defined through

$$
\chi^{(X)}(h):=\lim _{x \rightarrow x^{*}} \mathbb{P}\left(X_{h}>x \mid X_{o}>x\right),
$$

where $o \in \mathbb{R}^{d}$ denotes the origin. We refer to Chapter 3 for an introduction of TCFs as a proper subclass of positive definite kernels on $T \times T$. In the literature the TCF is considered an appropriate summary statistic for max-stable distributions, cf. [4, 10, 14, 28, 29, 85] among many others. Here, we explore to what extent the TCF can distinguish between different classes of max-stable processes. In fact, we identify essentially different stationary max-stable processes on $\mathbb{R}^{d}$ sharing the same TCF. The focus lies on the stationary max-stable processes presented in Section 1.2 and particular emphasis is put on radially symmetric TCFs that are monotonously decreasing as the radius grows. We start our analysis by identifying the TCFs of these basic models in Table 4.1. The expressions therein are verified or referenced in Section 4.5.1 (Proof of Table 4.1).

Remark 4.1.1. From the previous considerations we know already that any TCF can be realized by a spectrally discrete process as in Theorem 2.3.5 (cf. also (3.10). However, this model is irrelevant for spatial applications and therefore not considered 


\begin{tabular}{lll}
\hline Process model & Parameter & TCF $\chi(t)$ \\
\hline Brown-Resnick (BR) & variogram $\gamma$ & $\operatorname{erfc}(\sqrt{\gamma(t) / 8})$ \\
$\begin{array}{l}\text { Variance-mixed } \\
\text { Brown-Resnick (VBR) }\end{array}$ & $\begin{array}{l}\text { variogram } \gamma, \\
\text { distribution function } G \\
\text { on }(0, \infty) \text { with } G(0+)=0\end{array}$ & $\int_{0}^{\infty} \operatorname{erfc}\left(s \sqrt{\frac{\gamma(t)}{8}}\right) \mathrm{d} G(s)$ \\
\hline extremal Gaussian (EG) & correlation $\rho$ & $1-\sqrt{(1-\rho(t)) / 2}$ \\
$\begin{array}{l}\text { extremal binary Gaussian } \\
\text { (EBG) }\end{array}$ & correlation $\rho$ & $\pi^{-1} \arcsin \rho(t)+1 / 2$ \\
\hline Mixed Moving Maxima (M3) & random shape $f$ & $\mathbb{E}_{f} \iint_{\mathbb{R}^{d}} f(z) \wedge f(z-t) \mathrm{d} z$ \\
Mixed Poisson Storm (MPS) & $\begin{array}{l}\text { distribution function } F \\
\text { on }(0, \infty) \text { with } F(0+)=0\end{array}$ & $\mathcal{L}(F)\left(\frac{2 \kappa_{d-1}}{d \kappa_{d}}\|t\|_{2}\right)$ \\
\hline
\end{tabular}

Table 4.1.: Tail correlation functions $\chi(t)$ for $t \in \mathbb{R}^{d}$ of stationary max-stable processes on $\mathbb{R}^{d}$ from Section 1.2 The process models are grouped according to different long-range dependence. Here erfc denotes the complementary error function and $\mathcal{L}(F)$ the Laplace transform of the distribution function $F$. M3 processes are considered with a random shape function $f$.

here. For instance, it contains far too many parameters $\left(2^{m}-1\right.$ parameters for an $m$-variate distribution).

Comparable classes of TCFs If we want to compare the TCFs of processes in Table 4.1 we need to take into account that Brown-Resnick processes are processes associated to a variogram, which can be bounded or unbounded. The two situations - bounded and unbounded variogram - have to be treated separately. Due to their different behaviour towards long-range dependence (cf. Remark 1.2.4) that is reflected in the behaviour of the TCF $\chi(t)$ as $t \rightarrow \infty$ (cf. [50]), it is only meaningful to compare Brown-Resnick processes with bounded variogram to extremal Gaussian or extremal binary Gaussian processes and Brown-Resnick processes with unbounded variogram to Mixed Moving Maxima processes.

Certain monotonicity properties of functions on intervals (absolutely monotone, completely monotone, $\alpha$-times monotone) will enter the subsequent considerations and are therefore summarized in Section A.2. 


\subsection{Brown-Resnick vs. EG and EBG processes}

Comparing Brown-Resnick processes with extremal Gaussian and extremal binary Gaussian processes, we need to consider Brown-Resnick processes associated to bounded variograms on $\mathbb{R}^{d}$. Such variograms $\gamma$ are always of the form

$$
\gamma(t)=\lambda(1-\rho(t)) \quad t \in \mathbb{R}^{d}
$$

where $\rho$ is a correlation function on $\mathbb{R}^{d}$, and $\lambda>0$ (cf. [40, sect. 3.1] or [9, p. 32]). The respective TCF is $\operatorname{erfc}(\sqrt{\lambda(1-\rho) / 8})$ (cf. Table 4.1). On the other hand, the TCFs of an extremal Gaussian and an extremal binary Gaussian process are also functions of a correlation function $\rho$ (cf. Table 4.1). Thus, the ansatz

$$
\begin{array}{llrl}
\chi_{\mathrm{EG}}(t)=\chi_{\mathrm{EBG}}(t) & \Leftrightarrow & 1-\sqrt{\left(1-\rho_{\mathrm{EG}}(t)\right) / 2}=\pi^{-1} \arcsin \rho_{\mathrm{EBG}}(t)+1 / 2 \\
\chi_{\mathrm{BR}}^{(\lambda)}(t)=\chi_{\mathrm{EG}}(t) & \Leftrightarrow & \operatorname{erfc}\left[\sqrt{\lambda\left(1-\rho_{\mathrm{BR}}(t)\right) / 8}\right]=1-\sqrt{\left(1-\rho_{\mathrm{EG}}(t)\right) / 2} \\
\chi_{\mathrm{BR}}^{(\lambda)}(t)=\chi_{\mathrm{EBG}}(t) & \Leftrightarrow & \operatorname{erfc}\left[\sqrt{\lambda\left(1-\rho_{\mathrm{BR}}(t)\right) / 8}\right]=\pi^{-1} \arcsin \rho_{\mathrm{EBG}}(t)+1 / 2,
\end{array}
$$

leads to the question, if (or for which $\lambda>0$ ) the maps

$$
\begin{array}{rlrl}
R:[-1,1] & \rightarrow[-1,1], & R(x) & :=\cos (\pi \sqrt{(1-x) / 2}) \\
S_{\lambda}:[-1,1] & \rightarrow[-1,1], & S_{\lambda}(x):=1-2(\operatorname{erf}[\sqrt{\lambda(1-x) / 8}])^{2} \\
T_{\lambda}:[-1,1] \rightarrow[-1,1], & T_{\lambda}(x):=\cos (\pi \operatorname{erf}[\sqrt{\lambda(1-x) / 8}])
\end{array}
$$

(or its inverses $R^{-1}, S_{\lambda}^{-1}, T_{\lambda}^{-1}$ ) transform correlation functions again into correlation functions. (Here we write $\operatorname{erf}(x):=1-\operatorname{erfc}(x)$ for the error function.) Since convex combinations, products and (pointwise) limits of correlation functions are again correlation functions, this requirement will be met if these maps are continuous on $[-1,1]$ and analytic on $(-1,1)$, such that the respective Taylor series at 0 has only non-negative coefficients. Such functions are absolutely monotone on $[0,1]$ and conversely, the Taylor series representation at 0 of an absolutely monotone function on $[0,1]$ extends to $[-1,1]$. So in fact, we ask, whether or for which $\lambda$ these maps are analytic on $(-1,1)$ and absolutely monotone on $[0,1]$.

Proposition 4.2.1. The functions $R, S_{\lambda}$ and $T_{\lambda}=R \circ S_{\lambda}$ from (4.1), (4.2) and (4.3) are continuous on $[-1,1]$ and analytic on $(-1,1)$ for all $\lambda>0$.

a) The function $R-R(0)$ is absolutely monotone on $[0,1]$. 


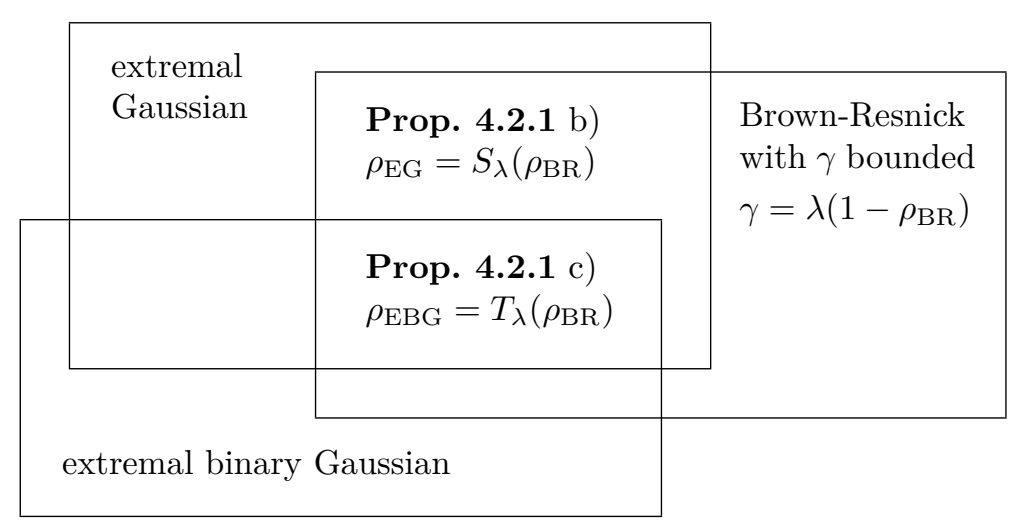

Figure 4.1.: Intersections of sets of tail correlation functions $\chi$ arising from extremal Gaussian (EG), extremal binary Gaussian (EBG) and Brown-Resnick processes (BR).

b) The function $S_{\lambda}$ is absolutely monotone on $[0,1]$ for $\lambda \leq 8\left(\operatorname{erf}^{-1}(1 / \sqrt{2})\right)^{2} \approx$ 4.425098 .

c) The function $T_{\lambda}=R \circ S_{\lambda}$ is absolutely monotone on $[0,1]$ for $\lambda \leq 8\left(\operatorname{erf}^{-1}(1 / 2)\right)^{2} \approx$ 1.8197 .

Thus, we have the following coincidences of classes of TCFs as summarized in Figure 4.1. Note that $T_{\lambda}=R \circ S_{\lambda}$ and the upper bound in Proposition 4.2.1 for $T_{\lambda}$ is smaller than the upper bound for $S_{\lambda}$, such that the transformation $T_{\lambda}$ gives rise only to elements in the intersection of all three classes of TCFs.

Example 4.2.2. The Brown-Resnick process on $\mathbb{R}^{d}$ associated to the variogram

$$
\gamma_{\mathrm{BR}}(t)=1.62\left(1-\exp \left(-\|t\|_{2}\right)\right) \quad t \in \mathbb{R}^{d}
$$

the extremal Gaussian process on $\mathbb{R}^{d}$ associated to the correlation function

$$
\rho_{\mathrm{EG}}(t)=1-2\left(\operatorname{erf}\left[0.45 \sqrt{1-\exp \left(-\|t\|_{2}\right)}\right]\right)^{2} \quad t \in \mathbb{R}^{d},
$$

and the extremal binary Gaussian process on $\mathbb{R}^{d}$ associated to the correlation function

$$
\rho_{\mathrm{EBG}}(t)=\cos \left(\pi \operatorname{erf}\left[0.45 \sqrt{1-\exp \left(-\|t\|_{2}\right)}\right]\right) \quad t \in \mathbb{R}^{d}
$$

all share the same TCF

$$
\chi(t)=\operatorname{erfc}\left[0.45 \sqrt{1-\exp \left(-\|t\|_{2}\right)}\right] \quad t \in \mathbb{R}^{d} .
$$


Indeed $\gamma_{\mathrm{BR}}$ is a well-known variogram on $\mathbb{R}^{d}$ (cf. e.g. [40, sect. 4]) and Proposition 4.2.1 ensures that the functions $\rho_{\mathrm{EG}}$ and $\rho_{\mathrm{EBG}}$ are correlation functions on $\mathbb{R}^{d}$, such that the respective processes are well-defined.

\subsection{Brown-Resnick vs. Mixed Moving Maxima processes}

Here, we restrict ourselves to stationary isotropic processes on $\mathbb{R}^{d}$. When comparing Brown-Resnick processes to M3 processes, we need to consider Brown-Resnick processes associated to unbounded variograms. In fact, we will consider only variograms that are radially symmetric around the origin $o \in \mathbb{R}^{d}$ and grow monotonously to $\infty$ as the radius grows. We will also involve variance-mixed BR processes and compare them to two kinds of M3 processes - M3 processes associated to radially symmetric non-increasing (random) shapes and Mixed Poisson storm processes. First, we identify the TCFs of these processes. Because we deal with stationary isotropic processes here, the involved TCFs will depend on the radius (Euclidean norm in $\mathbb{R}^{d}$ ) only, and it is convenient to treat them as functions on $[0, \infty)$.

\subsubsection{Identifying the classes}

Mixed Poisson storm processes The TCF of a Mixed Poisson storm process on $\mathbb{R}^{d}$ that is associated to the intensity mixing distribution function $F$ on $(0, \infty)$ (with $F(0+)=0)$ as in Example 1.2 .5 is given by the Laplace transform of $F$ as follows (cf. Table 4.1)

$$
\chi_{\mathrm{MPS}}(t)=\mathcal{L}(F)\left(\frac{2 \kappa_{d-1}}{d \kappa_{d}}\|t\|_{2}\right)=\int_{(0, \infty)} \exp \left(-\gamma \frac{2 \kappa_{d-1}}{d \kappa_{d}}\|t\|_{2}\right) \mathrm{d} F(\gamma) \quad t \in \mathbb{R}^{d}
$$

That means in every dimension $d$ the class of TCFs arising from Mixed Poisson storm processes coincides with the (radial) functions, which are completely monotone on $[0, \infty)$, take the value 1 at 0 and vanish at $\infty$ (cf. A.3). In particular, this class of functions does not depend on the specific dimension, even though the involved factor $2 \kappa_{d-1} /\left(d \kappa_{d}\right)$ does.

M3 processes of radial non-increasing shapes As we shall see next (Proposition 4.3.1), the class of TCFs of M3 processes on $\mathbb{R}^{d}$ of radial non-increasing (random) shapes can be identified as the Gneiting class $H_{d}$ (introduced below), which has already been studied intensively in [37]. In fact, the class $H_{d}$ can be realized as 
TCFs already by two special subclasses of M3 processes, namely M3 processes of ball indicator functions and Moving Maxima processes of a single (deterministic) shape function. To be more precise, we introduce the following notation: Let $B_{r}^{d}(o):=$ $\left\{h:\|h\|_{2} \leq r\right\} \subset \mathbb{R}^{d}$ denote the $d$-dimensional ball of radius $r$ centered at the origin and $\mathbf{1}_{B_{r}^{d}(o)}$ its indicator function. We define the following sets of (radial) functions:

$M_{d}$ - the set of TCFs of M3 processes on $\mathbb{R}^{d}$ of radial non-increasing shapes, i.e. $M_{d}$ comprises all functions of the form

$$
\chi\left(\|t\|_{2}\right)=\mathbb{E}_{f}\left(\int_{\mathbb{R}^{d}} f\left(\|z\|_{2}\right) \wedge f\left(\|z-t\|_{2}\right) \mathrm{d} z\right),
$$

where each realization of a random shape $f \geq 0$ depends on the radius only and is non-increasing in the radius, such that $\mathbb{E}_{f}\left(\int_{\mathbb{R}^{d}} f\left(\|t\|_{2}\right) \mathrm{d} t\right)=1$.

$M_{d}^{o} \quad$ - the subset of $M_{d}$, where the M3 process is in fact a Moving Maxima process with a deterministic shape function $f$.

$B_{d} \quad-$ the subset of $M_{d}$, where the M3 process has as shape functions only normalized indicator functions of balls $B_{R}^{d}(o)$, i.e.

$$
f\left(\|t\|_{2}\right)=1 / \nu_{d}\left(B_{R}^{d}(o)\right) \cdot \mathbb{1}_{\|t\|_{2} \leq R}
$$

with a random radius $R \in(0, \infty)$.

$H_{d} \quad-$ the Gneiting class (cf. [37, (17)]), i.e. the class of functions $\varphi$ on $[0, \infty)$ of the form

$$
\varphi(t)=\int_{(0, \infty)} h_{d}(s t) \mathrm{d} G(s)
$$

where $G$ is a distribution function on $(0, \infty)$ (with $G(0+)=0)$ and where

$$
h_{d}(t)=\frac{d \Gamma(d / 2)}{\sqrt{\pi} \Gamma((d+1) / 2)} \int_{t}^{1}\left(1-v^{2}\right)_{+}^{(d-1) / 2} \mathrm{~d} v .
$$

Here the function $h_{d}(t)=\tilde{h}_{d}(t) / \tilde{h}_{d}(0)$ with $\tilde{h}_{d}$ is the self-convolution of the ball indicator function $\mathbf{1}_{B_{0.5}^{d}(o)}$ viewed as a radial function and sometimes called Euclid's hat (cf. [37, 80]).

$V_{d} \quad-$ the Mittal-Berman class (for $d \geq 2$; cf. [37, (40)] and [64]), i.e. the class of functions $\varphi$ on $[0, \infty)$ of the form

$$
\varphi(t)=2 \int_{t / 2}^{\infty} \frac{S_{d, u, \theta(t, u)}}{S_{d, u, \pi}} p(u) \mathrm{d} u,
$$


where $p$ is a probability density function on $(0, \infty)$, such that $p(u) / u^{d-1}$ is non-increasing, and $S_{d, u, \theta}$ is the surface area of the sphere $\left\{x:\|x\|_{2}=\right.$ $u\} \subset \mathbb{R}^{d}$ intersected by the cone of angle $\theta(t, u)=\arccos (t /(2 u))$ (with apex the origin).

Gneiting [37] showed already that the two classes $H_{d}$ and $V_{d}$ coincide for $d \geq 2$. In fact, all of these classes coincide (see Section 4.5 for a proof).

Proposition 4.3.1. a) We have $M_{d}=M_{d}^{o}=B_{d}=H_{d}\left(=V_{d}\right)$ for $d \geq 1(d \geq 2)$.

b) In the equality $H_{d}=B_{d}$ the distribution function $G$ corresponds to the law of $1 /(2 R)$.

c) In the equality $H_{d}=M_{d}^{o}$ the deterministic shape function $f$ from $M_{d}^{o}$ and the distribution function $G$ from $H_{d}$ can be recovered from each other by

$$
f(u)=\frac{1}{b_{d}} \int_{0}^{1 /(2 u)} s^{d} \mathrm{~d} G(s) \quad \text { and } \quad G(s)=b_{d} \int_{0}^{s} \frac{1}{u^{d}} \mathrm{~d}\left[f\left(\frac{1}{2 u}\right)\right],
$$

where $b_{d}:=\nu_{d}\left(B_{0.5}^{d}(o)\right)=(\sqrt{\pi} / 2)^{d} / \Gamma(1+d / 2)$.

Thus, we can benefit from Gneitings analysis in [37, which is based on [100] and characterizes $H_{d}$ by monotonicity properties: The class $H_{1}$ consists precisely of the continuous symmetric functions on $\mathbb{R}$ (viewed as function on $[0, \infty)$ ), which are convex, take the value 1 at 0 and vanish at $\infty$. These functions are also known from Pólya's criterion (cf. [76, Theorem 1]), which states that elements in $H_{1}$ are positive definite. Now we know that these functions are even TCFs on $\mathbb{R}$. For $d \geq 2$ a function $\varphi:[0, \infty) \rightarrow \mathbb{R}$ belongs to the class $H_{d}$ if and only if $\varphi(0)=1, \varphi$ is continuous, $\lim _{t \rightarrow \infty} \varphi(t)=0$ and $-\varphi^{\prime}(\sqrt{\cdot})$ is $(d+1) / 2$-times monotone on $(0, \infty)$ (cf. [37, p. 103] and Definition A.2.6). In case $d \geq 3$ is odd, this has a nice geometric interpretation, which also reveals the connection to completely monotone functions when $d$ tends to $\infty$ (cf. [37, pp. 96]). The precise characterization of $H_{d}$ for $d \geq 2$ in terms of convexity properties is stated in [37, Theorems 3.1 and 3.3].

Moreover, [37, Theorems 3.2. and 3.4.] provide inversion formulas, how to gain the distribution function $G$ from a given $\varphi \in H_{d}$. If we want to recover also the defining quantities $f$ and $R$ of the classes $M_{d}^{o}$ and $B_{d}$ respectively, we additionally need to apply (4.8) or take into account that $G$ is the law of $1 /(2 R)$. The explicit expressions in case $d=1,2,3$ are given in Table 4.2 and derived in Section 4.5.1 (Proof of Table 4.2). This is of special interest to us, when we want to simulate the corresponding processes for a given TCF $\chi=\varphi$. 


\begin{tabular}{l|lll}
\hline & $d=1$ & $d=2$ & $d=3$ \\
\hline$f(u)$ & $-\chi^{\prime}(2 u)$ & $\frac{4 u}{\pi} \int_{0}^{1 /(2 u)} \sqrt{\frac{1}{(2 u t)^{2}}-1} \mathrm{~d} \lambda_{\chi}(t)$ & $\chi^{\prime \prime}(2 u) /(\pi u)$ \\
$G(s)$ & $\chi(1 / s)-\chi^{\prime}(1 / s) / s$ & $\frac{1}{6 s^{3}} \int_{0}^{s} \sqrt{(s / t)^{2}-1}\left(2(s / t)^{2}+1\right) \mathrm{d} \lambda_{\chi}(t)$ & $\int_{0}^{s} \frac{1}{3 t^{3}} \mathrm{~d} \lambda_{\chi}(t)$ \\
$g(s)$ & $\chi^{\prime \prime}(1 / s) / s^{3}$ & $\frac{1}{2 s^{4}} \int_{0}^{s} \sqrt{\frac{1}{(s / t)^{2}-1}} \mathrm{~d} \lambda_{\chi}(t)$ & $\lambda_{\chi}^{\prime}(s) /\left(3 s^{3}\right)$ \\
\hline
\end{tabular}

Table 4.2.: Recovery formulas for the monotone Moving Maxima shape function $f$, the distribution function $G$ of $S=1 /(2 R)$ and its density $g$ (if it exists) in dimensions $d=1,2,3$ from a given TCF $\chi$. The functions $f, G$ and $g$ are defined on $(0, \infty)$, where $f$ and $g$ may have a pole at 0 . We abbreviate $\lambda_{\chi}(t):=t \chi^{\prime \prime}(1 / t)$. In case $d=2$ we assume here that even $\chi \in H_{5}$ holds in order to eliminate an additional integral.

The classes $H_{d}$ are all nested, i.e. $H_{d} \supset H_{d+1}$ for all $d \in \mathbb{N}$. Gneiting [37] also characterizes the class

$$
H_{\infty}:=\bigcap_{d=1}^{\infty} H_{d}
$$

as scale mixtures of the complementary error function $\operatorname{erfc}(x)=\frac{2}{\sqrt{\pi}} \int_{x}^{\infty} e^{-y^{2}} \mathrm{~d} y$ (cf. [37. Theorems 3.7 and 3.8]). A function $\varphi:[0, \infty) \rightarrow \mathbb{R}$ belongs to the class $H_{\infty}$ if and only if it has an integral representation of the form

$$
\varphi(t):=\int_{(0, \infty)} \operatorname{erfc}(s t) \mathrm{d} G(s)
$$

for some distribution function $G$ on $(0, \infty)$ (with $G(0+)=0$ ). And this is the case if and only if $\varphi(0)=1, \varphi$ is continuous, $\lim _{t \rightarrow \infty} \varphi(t)=0$ and $-\varphi^{\prime}(\sqrt{\cdot})$ is completely monotone on $(0, \infty)$. In particular, this requirement of $-\varphi^{\prime}(\sqrt{\cdot})$ being completely monotone will be met if $\varphi$ itself is already completely monotone, since $\sqrt{\cdot}$ is a Bernstein function.

Variance-mixed Brown-Resnick processes The TCF $\chi$ of such a process $X$ associated to the variogram $\gamma$ and variance-mixing distribution $G$ as in Example 1.2.6 is given by

$$
\chi(t)=\int_{(0, \infty)} \operatorname{erfc}\left(s \sqrt{\frac{\gamma(t)}{8}}\right) \mathrm{d} G(s) .
$$




\begin{tabular}{lll}
\hline Distribution function $G(s)$ or $g(s)=G^{\prime}(s)$ & $\varphi(t)=\int_{0}^{\infty} \operatorname{erfc}(s t) \mathrm{d} G(s)$ & \\
\hline$G(s)=e^{-1 /(a s)^{2}}$ & $e^{-2 t / a}$ & $0<a$ \\
$g(s)=\frac{\sqrt{\pi}}{\Gamma(\nu) \Gamma\left(\frac{1}{2}-\nu\right)} \int_{0}^{s} \frac{x^{2 \nu-3} e^{-1 /\left(4 x^{2}\right)}}{\left(s^{2}-x^{2}\right)^{\nu+1 / 2}} \mathrm{~d} x$ & $\frac{2^{1-\nu}}{\Gamma(\nu)} t^{\nu} K_{\nu}(t)$ & $0<\nu<\frac{1}{2}$ \\
$G(s)=\operatorname{erf}(a s)$ & $1-\frac{2}{\pi} \arctan (t / a)$ & $0<a$ \\
$G(s)=1-e^{-(a s)^{2}}$ & $1-\left(1+(t / a)^{-2}\right)^{-1 / 2}$ & $0<a$ \\
\hline
\end{tabular}

Table 4.3.: Members of the Gneiting class $H_{\infty}$ (cf. $(4.9)$ ) and their corresponding distribution function $G(s)$ or probability density $g(s)=G^{\prime}(s)$ on $(0, \infty)$ as scale mixtures of the complementary error function. As special cases the exponential model, the Whittle-Matérn family, the arctan model and the Dagum model (cf. [6]) appear. Further explicit examples of members of the Gneiting class $H_{\infty}$ can be found in Table A.1.

Hence the TCFs of variance-mixed Brown-Resnick processes are precisely the functions of the form

$$
\chi(t)=\varphi\left(\sqrt{\frac{\gamma(t)}{8}}\right)
$$

for some $\varphi$ in the Gneiting class $H_{\infty}$ and some variogram on $\mathbb{R}^{d}$ (cf. 4.10). The Tables 4.3 and A.1 give examples of corresponding pairs $\varphi$ and distribution functions $G$ (or probability densities $g=G^{\prime}$ ) that we need to know when we want to simulate corresponding processes (see also Section 4.5.1. Proof of Table 4.3).

\subsubsection{Comparing the classes}

We summarize our findings of intersections of classes of TCFs from M3 processes and Brown-Resnick processes in Figure 4.2. So far, we have seen that the set of TCFs of M3 processes on $\mathbb{R}^{d}$ of radial non-increasing shapes coincides with the Gneiting class $H_{d}$ and can be realized already by either a deterministic shape function or a suitable mixture of normalized ball indicator functions (cf. Prop. 4.3.1). By definition (cf. (4.9) the class $H_{\infty}$ is part of each $H_{d}$ and $H_{\infty}$ comprises all completely monotone functions on $[0, \infty)$ that take the value 1 at 0 and vanish at $\infty$ (cf. [37, p. 115]). These completely monotone functions are in each dimension the TCFs of Mixed Poisson Storm processes. On the other hand, the class of TCFs of variance-mixed Brown-Resnick processes surely contains the TCFs of Brown-Resnick processes. 
Let us compare the TCFs from the Brown-Resnick constructions to the TCFs from the M3 processes. Since the TCFs of variance-mixed Brown-Resnick processes have the form $\chi(t)=\varphi(\sqrt{\gamma(t) / 8})$ for some variogram $\gamma$ on $\mathbb{R}^{d}$ and some $\varphi \in H_{\infty}$ (cf. (4.11), the whole class $H_{\infty}$ itself can be realized by such processes when we choose $\gamma(t)=8\|t\|_{2}^{2}$, which is a valid variogram in each dimension. Finally, we explain why the enumerated regions in Figure 4.2 are non-empty:

(A) and (B) The variogram $\gamma(t)=8\|t\|_{2}^{2 \alpha}$ is valid in each dimension for $\alpha \in(0,1]$ (corresponding to fractal Brownian motion). Hence $\operatorname{erfc}\left(t^{\alpha}\right)$ is a valid TCF of a Brown-Resnick process for $\alpha \in(0,1]$. Moreover, the function $\operatorname{erfc}\left(t^{\alpha}\right)$ belongs to $H_{\infty}$ for $\alpha \in(0,1]$ and it is even completely monotone for $\alpha \leq 0.5$.

(C) Consider the simple erfc-mixture

$$
\chi\left(\|t\|_{2}\right)=0.25 \cdot \operatorname{erfc}\left(\|t\|_{2}\right)+0.75 \cdot \operatorname{erfc}\left(5\|t\|_{2}\right) \quad t \in \mathbb{R}^{d} .
$$

Surely, $\chi$ is a member of $H_{\infty}$. Suppose that there is a Brown-Resnick process on $\mathbb{R}^{d}$ corresponding to a variogram $\tilde{\gamma}$ such that its TCF $\tilde{\chi}$ coincides with $\chi$. We will show now that this cannot be true for any dimension $d$. Otherwise,

$$
\tilde{\gamma}\left(\|t\|_{2}\right)=8\left[\operatorname{erfc}^{-1}\left(0.25 \cdot \operatorname{erfc}(\|t\|)+0.75 \cdot \operatorname{erfc}\left(5\|t\|_{2}\right)\right)\right]^{2} \quad t \in \mathbb{R}^{d}
$$

is a variogram for any dimension $d$. In particular, $\tilde{\gamma}\left(\|\cdot\|_{2}\right)$ is for any dimension $d$ a continuous negative definite function on $\mathbb{R}^{d}$. By $[5,5.1 .8]$ it follows that the function

$$
\psi(r)=\left[\operatorname{erfc}^{-1}(0.25 \cdot \operatorname{erfc}(\sqrt{r})+0.75 \cdot \operatorname{erfc}(5 \sqrt{r}))\right]^{2} \quad r \in[0, \infty)
$$

is a (continuous) negative definite function on $[0, \infty)$ in the semigroup sense and obviously $\psi(r) \geq 0$. Hence $\psi(r)$ is a Bernstein function (cf. [5, 4.4.3]). However, the second derivative of $\psi(r)$ has a local minimum. So, the assertion fails and our assumption must be wrong. That means there is a dimension $d_{0}$ such that the above $\chi \in H_{\infty}$ cannot be realized by a Brown-Resnick process for any dimension $d \geq d_{0}$ as a TCF.

(D) The class $H_{d}$ naturally contains functions with compact support, e.g. the function $h_{d}$ (cf. 4.5), whereas the class of TCFs of variance-mixed BR processes cannot contain such functions. To see this, recall 4.11 and observe that members of $H_{\infty}$ are scale mixtures of erfc that cannot have compact support. Thus, the involved variogram in 4.11 would have to take the value $\infty$ outside a compact region. 


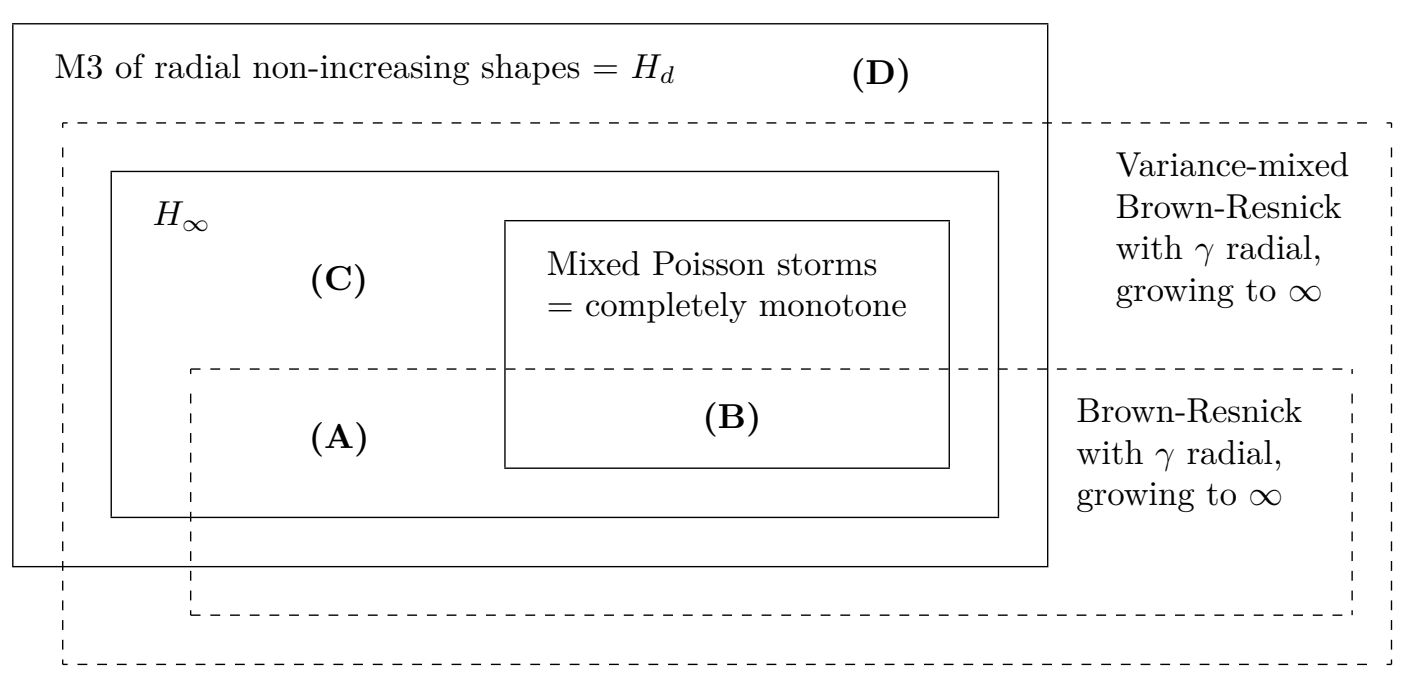

Figure 4.2.: Intersections of sets of tail correlation functions arising from Mixed Moving Maxima (M3) of radial non-increasing shapes, Mixed Poisson storms, Brown-Resnick and variance-mixed BR processes. Here, $H_{d}$ and $H_{\infty}$ refer to the Gneiting classes in (4.5) and (4.9). The Gneiting class $H_{d}$ can be realized as M3 process already by either a deterministic shape function or a suitable mixture of normalized ball indicator functions (cf. Prop. 4.3.1).

\subsubsection{Sharp bounds for parametric subclasses}

The considerations above also lead to sharp bounds for some well-known parametric families of positive definite functions to be a TCF, see Table 4.4

The first three families (powered exponential, Whittle-Matérn, Cauchy) are completely monotone for the respective parameters (cf. [63, (1.2),(1.6) and (2.32)] for example), and thus they can be realized by an M3 process of non-increasing shapes, by a Mixed Poisson storm process or by a variance-mixed BR process (in all cases in any dimension). The powered error function is not completely monotone, but a member of the Gneiting class $H_{\infty}$. That means it can be realized by an M3 process of non-increasing shapes or by a variance-mixed BR process (both in any dimension), but not by a Mixed Poisson storm process. In all of these cases, we may exclude bigger parameters $\nu$ because the (right-hand) derivative at 0 vanishes for bigger $\nu$, but the triangle inequality (3.14) enforces this derivative to be negative in order to be a TCF (cf. [58, Corollary 2] or [86, Theorem 3 (ii)]).

The truncated power function is an example of a TCF with compact support. Because a TCF has to be positive definite, this leads to the situation that the valid model parameter depends on the dimension. It is chosen such that the function belongs to $H_{d}$ (cf. [37, Theorem 6.3]), and thus can be realized by an M3 process of non-increasing shapes on $\mathbb{R}^{d}$. Because of its compact support the function cannot belong to any of the other classes presented in Figure 4.2 (cf. the comment on region 


\begin{tabular}{|c|c|c|}
\hline Parametric family & & TCF for \\
\hline powered exponential & $\exp \left(-r^{\nu}\right)$ & $0<\nu \leq 1$ \\
\hline Whittle-Matérn & $2^{1-\nu} \Gamma(\nu)^{-1} r^{\nu} K_{\nu}(r)$ & $0<\nu \leq 0.5$ \\
\hline Cauchy & $\left(1+r^{\nu}\right)^{-\beta} \quad \beta>0$ & $0<\nu \leq 1$ \\
\hline powered error function & $\operatorname{erfc}\left(r^{\nu}\right)$ & $0<\nu \leq 1$ \\
\hline truncated power function* & $(1-r)_{+}^{\nu}$ & $\nu \geq\lfloor d / 2\rfloor+1$ \\
\hline
\end{tabular}

Table 4.4.: Parametric families of radially symmetric functions on $\mathbb{R}^{d}$ and their sharp parameter bound for being a tail correlation function (TCF), cf. Figure $4.3{ }^{*}$ The bound for the truncated power function is sharp for odd dimensions.

(D)). The bound is sharp in odd dimensions because the function is not positive definite otherwise, cf. [41, Theorem 1 and p. 165]. For even dimensions this choice is valid, but possibly not sharp. Again due to [41, we know at least that $\nu$ has to satisfy $\nu \geq(d+1) / 2$ in order to be positive definite.

\subsection{Operations and Counterexamples}

From the previous considerations one might have the impression that any continuous radial TCF on $\mathbb{R}^{d}$ that is non-increasing and convex on $[0, \infty)$ and that vanishes at $\infty$, belongs to $H_{d}$ or at least appears already in Figure 4.2. This is true for $d=1$, since $H_{1}$ comprises all of these functions. The following operations however yield counterexamples for $d \geq 3$.

The turning bands operator has been inspired by [51] and is well-known in the context of isotropic Gaussian processes. Secondly, the multiplication with the Gneiting class $H_{d}$ can shorten the range of tail dependence to a compact set. These operations are derived from construction principles for the corresponding max-stable processes that can be applied to (almost arbitrary) spectral representations.

\subsubsection{Turning bands}

The turning bands operator Let $k, d \in \mathbb{N}$ with $1 \leq k \leq d$. The set of ordered tuples $\left(x_{1}, \ldots, x_{k}\right)$ of $k$ orthonormal vectors in $\mathbb{R}^{d}$ is known as the Stiefel manifold of orthonormal $k$-frames in $\mathbb{R}^{d}$ (cf. e.g. [68, p. 131]) and denoted $V_{k}\left(\mathbb{R}^{d}\right)$. If we 
interpret the vectors $x_{1}, \ldots, x_{k}$ as columns of a matrix, we identify

$$
V_{k}\left(\mathbb{R}^{d}\right)=\left\{A \in \mathbb{R}^{d \times k}: A^{\mathrm{T}} A=\mathbb{1}_{k \times k}\right\}
$$

where $A^{\mathrm{T}}$ denotes the transpose of $A$ and $\mathbb{1}_{k \times k}$ the identity matrix in $\mathbb{R}^{k \times k}$. A matrix $A \in V_{k}\left(\mathbb{R}^{d}\right)$ embeds $\mathbb{R}^{k}$ linearly and isometrically into $\mathbb{R}^{d}$, whereas $A^{\mathrm{T}}$ applied to a vector $t \in \mathbb{R}^{d}$ is a vector in $\mathbb{R}^{k}$ whose coordinates can be interpreted as the coordinates of the projection of $t$ onto $A\left(\mathbb{R}^{k}\right)$ with respect to the orthonormal frame defined by the columns of $A$. For $k=1$ the Stiefel manifold is simply the sphere $V_{1}\left(\mathbb{R}^{d}\right)=S^{d-1}$ and for $k=d$ the orthogonal group $V_{d}\left(\mathbb{R}^{d}\right)=\mathrm{O}(d)$.

In view of 4.12 the Stiefel manifold $V_{k}\left(\mathbb{R}^{d}\right)$ is a compact submanifold of $\mathbb{R}^{d \times k}$. The action of the orthogononal group $\mathrm{O}(d)$ (from the left) exhibits $V_{k}\left(\mathbb{R}^{d}\right)$ as a locally compact homogeneous space, on which a unique normalized left invariant Haar measure $\sigma_{k}^{d}$ can be defined [68, p. 142 Example 4], which we call uniform distribution [47, 57].

Definition 4.4.1. For $1 \leq k \leq d$ we define the turning bands operator by

$$
\mathrm{TB}_{k}^{d}: C\left(\mathbb{R}^{k}\right) \rightarrow C\left(\mathbb{R}^{d}\right) \quad \mathrm{TB}_{k}^{d}(f)(t):=\int_{V_{k}\left(\mathbb{R}^{d}\right)} f\left(A^{\mathrm{T}}(t)\right) \sigma_{k}^{d}(\mathrm{~d} A) .
$$

Indeed, the turning bands operator is well-defined, since $V_{k}\left(\mathbb{R}^{d}\right)$ is compact.

Lemma 4.4.2. Let $k_{1} \leq k_{2} \leq k_{3}$.

a) The composition map

$$
V_{k_{1}}\left(\mathbb{R}^{k_{2}}\right) \times V_{k_{2}}\left(\mathbb{R}^{k_{3}}\right) \rightarrow V_{k_{1}}\left(\mathbb{R}^{k_{3}}\right) \quad(A, B) \mapsto B \circ A
$$

is continuous.

b) If $B \sim \sigma_{k_{2}}^{k_{3}}$ is uniformly distributed on $V_{k_{2}}\left(\mathbb{R}^{k_{3}}\right)$ and $A$ is an independent (Borelmeasurable) random variable with values in $V_{k_{1}}\left(\mathbb{R}^{k_{2}}\right)$, then the composition $B \circ A$ will also be uniformly distributed $B \circ A \sim \sigma_{k_{1}}^{k_{3}}$.

c) The turning bands operator is compatible with compositions

$$
\mathrm{TB}_{k_{1}}^{k_{2}} \circ \mathrm{TB}_{k_{2}}^{k_{3}}=\mathrm{TB}_{k_{1}}^{k_{3}}
$$

In the context of Gaussian processes and positive definite functions, the turning bands operator $\mathrm{TB}_{1}^{d}$ is a familiar operator, see [38, 39, 59, 84, 103], where explicit formulas and recurrence relations are provided. Let $\Phi_{d}$ denote the set of radially 
symmetric continuous correlation functions on $\mathbb{R}^{d}$. Then it is well-known that $\mathrm{TB}_{1}^{d}$ yields a bijection between $\Phi_{1}$ and $\Phi_{d}$. In view of 4.13 this implies that $\mathrm{TB}_{k}^{d}$ is a bijection between $\Phi_{k}$ and $\Phi_{d}$. The operator $\operatorname{TB}_{k}^{d}$ for arbitrary $k, d \in \mathbb{N}$ with $k \leq d$ is usually implicitly addressed as $\mathrm{TB}_{1}^{d} \circ\left(\mathrm{TB}_{1}^{k}\right)^{-1}$ in the references above. Because of these bijections, the turning bands method is an important tool for the simulation of stationary isotropic Gaussian processes.

In the context of max-stable processes and their TCFs the situation transfers to the following extent.

The turning bands method for max-stable processes Let $X$ be a stochastically continuous simple max-stable process on $\mathbb{R}^{k}$. Then the process $X$ has a spectral representation (1.4)

$$
X_{t}=\bigvee_{n=1}^{\infty} U_{n} V_{t}\left(\omega_{n}\right) \quad t \in \mathbb{R}^{k}
$$

where $\left\{\left(U_{n}, \omega_{n}\right)\right\}_{n=1}^{\infty}$ denotes a Poisson point process on $\mathbb{R}_{+} \times \Omega$ with intensity $u^{-2} \mathrm{~d} u \nu(\mathrm{d} \omega)$ and the spectral function $V_{t}(\omega)$ is jointly measurable in the variables $t \in \mathbb{R}^{k}$ and $\omega \in \Omega$. Based on this representation we define another simple max-stable process $Y$ on $\mathbb{R}^{d}$ with $d \geq k$ as follows. Let $\left(U_{n}, \omega_{n}, A_{n}\right)$ be a Poisson point process on $\mathbb{R} \times \Omega \times V_{k}\left(\mathbb{R}^{d}\right)$ of intensity $u^{-2} \mathrm{~d} u \nu(\mathrm{d} \omega) \sigma_{k}^{d}(\mathrm{~d} A)$, where $\sigma_{k}^{d}(\mathrm{~d} A)$ is the uniform distribution on the Stiefel manifold $V_{k}\left(\mathbb{R}^{d}\right)$. Set

$$
Y_{t}:=\bigvee_{n=1}^{\infty} U_{n} V_{A_{n}^{\mathrm{T}}(t)}\left(\omega_{n}\right) \quad t \in \mathbb{R}^{d}
$$

Then $Y$ is a simple max-stable process on $\mathbb{R}^{d}$ with the following properties.

Lemma 4.4.3. Let $X$ and $Y$ be simple max-stable processes as given by (4.14) and (4.15) respectively.

a) If $X$ is stationary, then $Y$ is stationary.

b) For any $G \in \mathrm{O}(d)$ the law of $\left\{Y_{G(t)}\right\}_{t \in \mathbb{R}^{d}}$ and the law of $Y$ coincide.

c) Let $X$ be stationary. The (radial) $T C F \chi^{(Y)}$ of the stationary isotropic process $Y$ can be expressed in terms of the TCF $\chi^{(X)}$ of $X$ by

$$
\chi^{(Y)}=\operatorname{TB}_{k}^{d}\left(\chi^{(X)}\right)
$$


Proposition 4.4.4. If $\chi$ is a continuous $T C F$ on $\mathbb{R}^{k}$, then $\operatorname{TB}_{k}^{d}(\chi)$ is a continuous $T C F$ on $\mathbb{R}^{d}$.

However, not all radially symmetric continuous $\mathrm{TCFs}$ on $\mathbb{R}^{d}$ arise as $\mathrm{TB}_{k}^{d}(\chi)$ for some TCF $\chi$ on $\mathbb{R}^{k}$. As a counterexample consider the identity

$$
\exp (-t)=\mathrm{TB}_{1}^{3}(f)(t) \quad \text { with } \quad f(t)=\frac{\mathrm{d}}{\mathrm{d} t}(t \exp (-t))=(1-t) \exp (-t)
$$

(cf. [84, (2.22)]). While the completely monotone function $\exp (-t)$ is a valid radial TCF on $\mathbb{R}^{3}$, the function $f$ cannot be a TCF on $\mathbb{R}$ since $f$ attains negative values. Therefore, it is necessary to consider $\mathrm{TB}_{k}^{d}$ also for $k>1$ (and not only $k=1$ ) in the context of radial TCFs.

Remark 4.4.5. The turning bands method is compatible with iterations in the following sense: Let $q \geq d$ and construct a process $Z$ on $\mathbb{R}^{q}$ from the spectral representation of $Y$ on $\mathbb{R}^{d}$ by

$$
Z_{t}:=\bigvee_{n=1}^{\infty} U_{n} V_{B_{n}^{\mathrm{T}} \circ A_{n}^{\mathrm{T}}(t)}\left(\omega_{n}\right)=\bigvee_{n=1}^{\infty} U_{n} V_{\left(A_{n} \circ B_{n}\right)^{\mathrm{T}}(t)}\left(\omega_{n}\right) \quad t \in \mathbb{R}^{q},
$$

where $\left(U_{n}, \omega_{n}, A_{n}, B_{n}\right)$ is a Poisson point process on $\mathbb{R} \times \Omega \times V_{k}\left(\mathbb{R}^{d}\right) \times V_{d}\left(\mathbb{R}^{q}\right)$ with intensity $u^{-2} \mathrm{~d} u \nu(\mathrm{d} \omega) \sigma_{k}^{d}(\mathrm{~d} A) \sigma_{d}^{q}(\mathrm{~d} B)$. Then Lemma 4.4.2 implies that $Z=\left\{Z_{t}\right\}_{t \in \mathbb{R}^{q}}$ has the same law as

$$
\left\{\bigvee_{n=1}^{\infty} U_{n} V_{C_{n}^{\mathrm{T}}(t)}\left(\omega_{n}\right)\right\}_{t \in \mathbb{R}^{q}}
$$

where $\left(U_{n}, \omega_{n}, C_{n}\right)$ is a Poisson point process with intensity $u^{-2} \mathrm{~d} u \nu(\mathrm{d} \omega) \sigma_{k}^{q}(\mathrm{~d} C)$. Thus, the process $Z$ can be constructed directly from the spectral representation of $X$ without involving $Y$ as a step in between.

\subsubsection{Multiplication with the Gneiting class $H_{d}$}

Let $X$ be a stochastically continuous max-stable process on $\mathbb{R}^{d}$ with spectral representation as in 4.14 with $k=d$ and let $\{B(t)\}_{t \in \mathbb{R}^{d}}$ be a measurable process on $\mathbb{R}^{d}$ taking values in $\{0,1\}$. We denote the probability space corresponding to $B$ by $\left(\Omega_{B}, \mathcal{A}_{B}, \mathbb{P}_{B}\right)$ and expectation w.r.t. $\mathbb{P}_{B}$ by $\mathbb{E}_{B}$. Further, we require that

$$
c_{B}:=\int_{\mathbb{R}^{d}} B(t) \mathrm{d} t \in(0, \infty)
$$


holds $\mathbb{P}_{B}$-almost surely. Based on these two processes $X$ and $B$ we define another simple max-stable process $Y$ on $\mathbb{R}^{d}$ by

$$
Y_{t}:=\bigvee_{n=1}^{\infty} U_{n} \frac{B_{n}\left(t-z_{n}\right)}{c_{B_{n}}} V_{t}\left(\omega_{n}\right) \quad t \in \mathbb{R}^{d},
$$

where $\left(U_{n}, \omega_{n}, z_{n}, B_{n}\right)$ is a Poisson point process on $\mathbb{R} \times \Omega \times \mathbb{R}^{d} \times \Omega_{B}$ with intensity $u^{-2} \mathrm{~d} u \nu(\mathrm{d} \omega) \mathrm{d} z \mathbb{P}_{B}\left(\mathrm{~d} \omega_{B}\right)$.

Lemma 4.4.6. Let $X$ and $Y$ be simple max-stable processes as given by 4.14) for $k=d$ and (4.16) respectively.

a) If $X$ is stationary, then $Y$ is stationary.

b) Let $X$ be stationary. The TCF $\chi^{(Y)}$ of the stationary process $Y$ can be expressed in terms of the TCF $\chi^{(X)}$ of $X$ by

$$
\chi^{(Y)}(t)=\mathbb{E}_{B}\left[\frac{\int_{\mathbb{R}^{d}} B(z) B(z-t) \mathrm{d} z}{\int_{\mathbb{R}^{d}} B(z) \mathrm{d} z}\right] \cdot \chi^{(X)}(t) \quad t \in \mathbb{R}^{d} .
$$

Example 4.4.7. Let $X$ be a stationary stochastically continuous max-stable process on $\mathbb{R}^{d}$ and let $R \in(0, \infty)$ be a random radius. Set $B(t):=\mathbb{1}_{\|t\|_{2} \leq R}$ the indicator function of the ball $B_{R}^{d}(o)$. Construct the process $Y$ as in 4.16 . Then Lemma 4.4.6 and Proposition 4.3.1 give

$$
\chi^{(Y)}(t)=\varphi_{G}\left(\|t\|_{2}\right) \cdot \chi^{(X)}(t) \quad t \in \mathbb{R}^{d},
$$

where the function $\varphi_{G}$ belongs to the Gneiting class $H_{d}$ corresponding to the distribution function $G$ of $1 /(2 R)$.

Remark 4.4.8. From Proposition 3.3.1, it is already known that multiplication of TCFs on some space yields again TCFs on the same space. The advantage here is the explicit construction of a max-stable process from a given spectral representation.

\subsubsection{Counterexamples}

First, we provide for each $d \geq 3$ an example of a radial continuous TCF on $\mathbb{R}^{d}$ that is convex on $[0, \infty)$ and vanishes at $\infty$ (which is to say that it belongs to $H_{1}$ ), but does not belong to the Gneiting class $H_{d}$ (cf. (4.5)). Therefore, consider the basic function of the class $H_{1}$, the tent function

$$
h_{1}(t)=(1-t)_{+} \quad t \geq 0 .
$$


Surely, the tent function $h_{1} \in M_{1}$ is a radial TCF on $\mathbb{R}$ that can be realized by an M3 process (cf. Prop. 4.3.1). If we apply the turning bands operator we obtain

$$
\varphi_{d}(t):=\mathrm{TB}_{1}^{d}\left(h_{1}\right)(t) \quad t \geq 0
$$

which is a radial TCF on $\mathbb{R}^{d}$ (cf. Proposition 4.4.4). By [38, equation (6)] $\varphi_{d}$ can be expressed as

$$
\varphi_{d}(t)=\frac{2 \Gamma(d / 2)}{\sqrt{\pi} \Gamma((d-1) / 2)} \int_{0}^{1} h_{1}(t w)\left(1-w^{2}\right)^{(d-3) / 2} \mathrm{~d} w
$$

Proposition 4.4.9. Let $\varphi_{d}$ be the function from (4.17).

a) For $d \geq 1$ the function $\varphi_{d}$ is a radial TCF on $\mathbb{R}^{d}$ that belongs to $H_{1}$.

b) For $d \geq 1$ the function $\varphi_{d}$ does not belong to $H_{k}$ for $k \geq 3$.

c) For $d=1$ and $d \geq 6$ the function $\varphi_{d}$ does not belong to $\mathrm{H}_{2}$.

Remark 4.4.10. In the remaining cases $d \in\{2,3,4,5\}$ plots of the function $c(t)$ from (4.23) suggest that $\varphi_{d}$ does not belong to $H_{2}$ either, since $c(t)$ is not convex, see Figure 4.4 in Section 4.5 .2 .

Remark 4.4.11. The function $\varphi_{d}$ decreases linearly on the interval $[0,1](\mathrm{cf}$. 4.22)

$$
\varphi_{d}(t)=1-\beta_{d} \cdot t \quad 0 \leq t \leq 1, \quad \text { where } \quad \beta_{d}=\frac{\Gamma(d / 2)}{\sqrt{\pi} \Gamma((d+1) / 2)} .
$$

Therefore, the radial function $\chi(t):=1-\beta \cdot t$ is an admissible radial TCF on the $d$-dimensional ball of radius $r$ if $\beta \in\left[0, \beta_{d} / r\right]$. This complements results in [38, where it is shown that $\varphi(t)=1-\alpha t$ is positive definite on the $d$-dimensional ball of radius $r$ if and only if $\alpha \in\left[0,2 \beta_{d} / r\right]$.

Secondly, combing the turning bands operator and the multiplication operation leads to an example of a radial TCF on $\mathbb{R}^{3}$ that is convex on $[0, \infty)$ and vanishes at $\infty$, but that is not contained in any of the classes given in Figure 4.2 for $d=3$. Therefore, consider the function

$$
\chi_{d}(t):=\varphi_{d}(2 t) \cdot h_{d}(t) \quad t \geq 0
$$

where $\varphi_{d}$ is from 4.17.

Proposition 4.4.12. Let $\chi_{d}$ be the function from (4.20).

a) For $d \geq 1$ the function $\chi_{d}$ is a radial TCF on $\mathbb{R}^{d}$ that belongs to $H_{1}$. 
b) For $d \geq 1$ the function $\chi_{d}$ does not belong to the class of TCFs of variance-mixed $B R$ processes on $\mathbb{R}^{d}$.

c) The function $\chi_{3}$ does not belong to any of the classes given in Figure 4.2 for $d=3$.

Remark 4.4.13. It seems very likely that part (c) of Proposition 4.4 .12 is also true for general $d \geq 2$.

\subsection{Proofs and plots}

To deal with the function $S_{\lambda}$ in Proposition 4.2.1. we first prove an auxiliary lemma, which might be interesting in its own right.

Lemma 4.5.1. The function $f(x)=1-(\operatorname{erf}(\sqrt{x}))^{2}$ is completely monotone on $[0, \infty)$.

Proof. The function $f$ is non-negative, continuous on $[0, \infty)$ and the first derivative of $f$ on $(0, \infty)$ is given by

$$
f^{\prime}(x)=-\frac{2}{\sqrt{\pi}} \frac{\operatorname{erf}(\sqrt{x})}{\sqrt{x}} \cdot e^{-x} \quad x>0 .
$$

Now, the functions $e^{-x}$ and $\operatorname{erf} \sqrt{x} / \sqrt{x}$ are completely monotone on $(0, \infty)$ (cf. $[63$, (1.2) and Corollary to Theorem 5]). Hence, $-f^{\prime}$ is completely monotone, which shows that $f$ is completely monotone on $[0, \infty)$.

Proof of Proposition 4.2.1. (Parts a) and c) and a first version of part b) are due to Felix Ballani.)

It can be seen directly that the functions $R, S_{\lambda}, T_{\lambda}$ are continuous on $[-1,1]$ and analytic on $(-1,1)$ for all $\lambda>0$.

a) Using the series expansion of the cosine function, we arrive at

$$
\begin{aligned}
& R(x)=\sum_{n=0}^{\infty} \frac{(-1)^{n}}{(2 n) !} \pi^{2 n} \frac{(1-x)^{n}}{2^{n}}=\sum_{n=0}^{\infty} \frac{\pi^{2 n}}{2^{n}(2 n) !} \sum_{k=0}^{n}\left(\begin{array}{c}
n \\
k
\end{array}\right) x^{k}(-1)^{n-k} \\
& =\sum_{k=0}^{\infty} x^{k} \sum_{n=0}^{\infty} \frac{\pi^{2 n+2 k}}{2^{n+k}(2 n+2 k) !}\left(\begin{array}{c}
n+k \\
k
\end{array}\right)(-1)^{n} \\
& =R(0)+\sum_{k=1}^{\infty} x^{k} \frac{\pi^{2 k}}{2^{2 k} k !} \sum_{n=0}^{\infty}(-1)^{n} \frac{\pi^{2 n}}{2^{n}(2 n) !} \frac{1}{(2 n+2 k-1)(2 n+2 k-3) \ldots(2 n+1)}
\end{aligned}
$$


and it suffices to check, whether the coefficients

$$
a_{k}:=\sum_{n=0}^{\infty}(-1)^{n} \frac{\pi^{2 n}}{2^{n}(2 n) !} \frac{1}{(2 n+2 k-1)(2 n-2 k-3) \cdots(2 n+1)}
$$

are non-negative for $k \geq 1$. Since this series representing $a_{k}$ converges absolutely, we may partition by even $(n=2 \ell)$ and odd $(n=2 \ell+1)$ coefficients:

$$
\begin{aligned}
a_{k}= & \sum_{\ell=0}^{\infty} \frac{\pi^{4 \ell}}{2^{2 \ell}(4 \ell) !} \frac{1}{(4 \ell+2 k-1) \cdots(4 \ell+1)} \\
& -\sum_{\ell=0}^{\infty} \frac{\pi^{4 \ell+2}}{2^{2 \ell+1}(4 \ell+2) !} \frac{1}{(4 \ell+2 k+1) \cdots(4 \ell+3)} \\
= & \sum_{\ell=0}^{\infty} \frac{\pi^{4 \ell}}{2^{2 \ell}(4 \ell) !} \frac{1}{(4 \ell+2 k-1) \cdots(4 \ell+3)}\left[\frac{1}{4 \ell+1}-\frac{\pi^{2} / 2}{(4 \ell+2)(4 \ell+1)} \frac{1}{4 \ell+2 k+1}\right]
\end{aligned}
$$

Now, the expression in the brackets is positive since $k \geq 1$ and $\ell \geq 0$. Thus, $a_{k}>0$ for $k \geq 1$. In particular, $R(x)-R(0)$ is absolutely monotone on $[0,1]$.

b) Lemma 4.5.1 tells us that $f(x)=1-(\operatorname{erf}(\sqrt{x}))^{2}$ is completely monotone on $[0, \infty)$. Now, $S_{\lambda}(x)=2 f(\lambda(1-x) / 8)-1$. Hence, the $k$-th derivative for $k \geq 1$ satisfies

$$
S_{\lambda}^{(k)}(x)=2\left(\frac{\lambda}{8}\right)^{k}(-1)^{k} f^{(k)}\left(\frac{\lambda}{8}(1-x)\right) \geq 0 .
$$

In particular, all but eventually the 0 -th Taylor coefficient $S_{\lambda}(0)$ are non-negative, and $S_{\lambda}(0)$ is non-negative if and only if $\lambda \leq 8\left(\operatorname{erf}^{-1}(1 / \sqrt{2})\right)^{2}$.

c) Since $T_{\lambda}=R \circ S_{\lambda}$ and $T_{\lambda}^{\prime}=\left(R^{\prime} \circ S_{\lambda}\right) \cdot S_{\lambda}^{\prime}$, it follows from the proof of a and b) that all but eventually the 0 -th Taylor coefficient $T_{\lambda}(0)$ are non-negative, and $T_{\lambda}(0)$ is non-negative if and only if $\lambda \leq 8\left(\operatorname{erf}^{-1}(1 / 2)\right)^{2}$.

Proof of Proposition 4.3.1. We divide the proof into five steps:

1st step $H_{d}=B_{d}$ for $d \geq 1$.

By definition, members of the class $B_{d}$ have the form

$$
\chi\left(\|t\|_{2}\right)=\mathbb{E}_{R}\left(\frac{1}{\nu_{d}\left(B_{R}^{d}(o)\right)} \int_{\mathbb{R}^{d}} \mathbb{1}_{\|z\|_{2} \leq R} \wedge \mathbb{1}_{\|z-t\|_{2} \leq R} \mathrm{~d} z\right)=\mathbb{E}_{R}\left(h_{d}\left(\frac{\|t\|_{2}}{2 R}\right)\right)
$$

for some random radius $R \in(0, \infty)$. The last equality holds, because the integral with the minimum $\wedge$ is in fact a convolution for indicator functions. Therefore, the transformation $S:=1 /(2 R)$ shows that this $\chi$ and $\varphi$ from 4.5 
are equal, when $G$ denotes the law of $S$ on $(0, \infty)$ and vice versa. Hence $B_{d}=H_{d}$ for $d \geq 1$.

2nd step $M_{d}^{o}=V_{d}=H_{d}$ for $d \geq 2$ and (4.8) holds for $d \geq 2$.

Members of $M_{d}^{o}$ depend on a shape function $f \geq 0$ with $\int_{\mathbb{R}^{d}} f\left(\|t\|_{2}\right) \mathrm{d} t=1$, which is non-increasing as the radius grows, whereas members of $V_{d}$ depend on a probability density function $p$ on $(0, \infty)$ with $p(u) / u^{d-1}$ non-increasing in $u>0$. Integration along the radius shows that both functions are in 1:1corresponcence via

$$
f\left(\|t\|_{2}\right)=\frac{p\left(\|t\|_{2}\right)}{S_{d,\|t\|_{2}, \pi}}
$$

Moreover, since $f$ is non-increasing, this correspondence is compatible with the integration in 4.7) and 4.4 with deterministic $f$. Hence $M_{d}^{o}=V_{d}$ for $d \geq 2$. From [37] we already know that $H_{d}=V_{d}$. In particular, $f$ and $G$ can also be recovered from each other by (44) and (45) in [37] with $n \geq 2$ or 4.8 with $d \geq 2$ here (where our $f$ corresponds to $g$ therein).

3rd step $M_{1}^{o}=H_{1}$ and 4.8 holds for $d=1$.

If $d=1$, it is straightforward to check that for $\chi \in M_{1}^{o}$ depending on a single shape function $f$, we have

$$
\chi(t)=\int_{\mathbb{R}} f(z) \wedge f(z-t) \mathrm{d} z=2 \int_{t / 2}^{\infty} f(u) \mathrm{d} u
$$

(similarly to the integration along the radius in (4.7)). Now, precisely the same proof as the proof of Theorem 5.2. in [37] applies here, when we set $n=1$, $g=f, \varphi=\chi$ and omit the term $S_{n, u, \theta}$ in (48) and (49) therein, showing that $M_{1}^{o}=H_{1}$. In particular, $f$ and $G$ can also be recovered from each other by (44) and (45) in [37] with $n=1$ or 4.8 with $d=1$ here (where our $f$ corresponds to $g$ therein).

4th step $M_{d} \subset H_{d}$ for $d \geq 1$.

From the 2nd and 3 rd step we know that $M_{d}^{o}=H_{d}$ for $d \geq 1$. That means for each (single deterministic) radially symmetric non-increasing shape function $f \geq 0$ on $\mathbb{R}^{d}$ with $0<\|f\|_{L^{1}\left(\mathbb{R}^{d}\right)}<\infty$ we may define a unique distribution function $G_{f /\|f\|_{\mathrm{L}^{1}\left(\mathbb{R}^{d}\right)}}$ via 4.8 . We set

$$
A(f)_{s}:=\|f\|_{\mathrm{L}^{1}\left(\mathbb{R}^{d}\right)} \cdot G_{f /\|f\|_{\mathrm{L}^{1}\left(\mathbb{R}^{d}\right)}}(s) \quad s>0
$$


such that $A(f)$ is non-decreasing on $(0, \infty)$ with $A(f)_{0+}=0$, right-continuous and $A(f)$ has total variation $\|f\|_{\mathrm{L}^{1}\left(\mathbb{R}^{d}\right)}$. It is coherent to set $A(0) \equiv 0$. Now, consider a member $\chi$ of $M_{d}$ and its corresponding measurable process $\{f(t)\}_{t \in \mathbb{R}^{d}}$, which satisfies $\mathbb{E}_{f}\left(\|f\|_{\mathrm{L}^{1}\left(\mathbb{R}^{d}\right)}\right)=1$. Then $\left\{A(f)_{s}\right\}_{s>0}$ defines a nondecreasing, right-continuous process with $\mathbb{E}\left(A(f)_{\infty}\right)=1$ and $A(f)_{0+}=0$. Moreover, note that (by the correspondence $M_{d}^{o}=H_{d}$ )

$$
\chi(t)=\mathbb{E}_{f}\left(\int_{0}^{\infty} h_{d}(s t) \mathrm{d} A(f)_{s}\right) .
$$

Set $G(s):=\mathbb{E}_{f} A(f)_{s}$. Then $G$ is also non-decreasing, right-continuous with total variation 1 and with $G(0+)=0$ (by dominated convergence). Finally, we obtain again by dominated convergence that

$$
\chi(t)=\int_{0}^{\infty} h_{d}(s t) \mathrm{d} \mathbb{E}_{f} A(f)_{s}=\int_{0}^{\infty} h_{d}(s t) \mathrm{d} G(s)
$$

as desired. Hence $M_{d} \subset H_{d}$.

5th step (Summary) From the previous steps we know that $M_{d} \subset H_{d}=B_{d}=M_{d}^{o}$ for $d \geq 1$. Clearly, $B_{d} \subset M_{d}$ by definition, so that $M_{d}, M_{d}^{o}, B_{d}, H_{d}$ coincide for $d \geq 1$. The equality $V_{d}=H_{d}$ for $d \geq 2$ is already known from [37.

Proof of Lemma 4.4.2. a) The composition of matrices is continuous and here just restricted to a subspace.

b) Let $f$ be a continuous function on $V_{k_{1}}\left(\mathbb{R}^{k_{3}}\right)$, then (by dominated convergence) the function $g(b):=\mathbb{E}_{A}(f(b \circ A))$ will also be continuous on $V_{k_{2}}\left(\mathbb{R}^{k_{3}}\right)$. Therefore, $\mathbb{E}_{B}\left(g\left(G^{-1} B\right)\right)=\mathbb{E}_{B}(g(B))$ for all $G \in \mathrm{O}\left(k_{3}\right)$, since $B \sim \sigma_{k_{2}}^{k_{3}}$. Thus, we also have for $G \in \mathrm{O}\left(k_{3}\right)$ that

$$
\begin{aligned}
\mathbb{E} f\left(G^{-1} \circ B \circ A\right) & =\mathbb{E}\left(\mathbb{E}\left(f\left(G^{-1} \circ B \circ A\right) \mid B\right)\right)=\mathbb{E}\left(g\left(G^{-1} B\right)\right) \\
& =\mathbb{E}(g(B))=\mathbb{E}(\mathbb{E}(f(B \circ A) \mid B))=\mathbb{E} f(B \circ A) .
\end{aligned}
$$

c) The assertion follows from part b).

Proof of Lemma 4.4.3. Let $M$ be a non-empty finite subset of $\mathbb{R}^{d}$ and $x \in(0, \infty)^{M}$. The f.d.d. of $Y$ are determined by

$$
-\log \mathbb{P}\left(Y_{t} \leq x_{t}, t \in M\right)=\int_{V_{k}\left(\mathbb{R}^{d}\right)} \int_{\Omega}\left(\bigvee_{t \in M} \frac{V_{A^{\mathrm{T}} t}(\omega)}{x_{t}}\right) \nu(\mathrm{d} \omega) \sigma_{k}^{d}(\mathrm{~d} A) .
$$


a) If $X$ is stationary, then

$$
\int_{\Omega}\left(\bigvee_{t \in M} \frac{V_{A^{\mathrm{T}}(t+h)}(\omega)}{x_{t}}\right) \nu(\mathrm{d} \omega)=\int_{\Omega}\left(\bigvee_{t \in M} \frac{V_{A^{\mathrm{T}} t}(\omega)}{x_{t}}\right) \nu(\mathrm{d} \omega)
$$

for all $h \in \mathbb{R}^{d}$ and all $A \in V_{k}\left(\mathbb{R}^{d}\right)$, since $A$ is linear.

b) This follows since $\sigma_{k}^{d}$ is $\mathrm{O}(d)$-invariant.

c) The assertion follows from 3.2 .

Proof of Proposition 4.4.4. In view of Lemma 4.4.3 we need to show that continuous TCFs on $\mathbb{R}^{k}$ coincide with the TCFs of stochastically continuous processes on $\mathbb{R}^{k}$. Therefore, let $\chi$ be a continuous TCF on $\mathbb{R}^{k}$ and let $X$ be a corresponding stationary max-stable process. Let $\theta$ be the ECF of $X$ and let $X^{*}$ be the simple max-stable process associated to $\theta$ as in Theorem 2.3.5. Note that $\chi(h)=2-\theta(\{h, o\})$. By construction, $X^{*}$ is also stationary and has TCF $\chi$. Additionally, $X^{*}$ is stochastically continuous due to Theorem 2.5.4.

Proof of Lemma 4.4.6. Let $M$ be a non-empty finite subset of $\mathbb{R}^{d}$ and $x \in(0, \infty)^{M}$. The f.d.d. of $Y$ are determined by

$$
-\log \mathbb{P}\left(Y_{t} \leq x_{t}, t \in M\right)=\mathbb{E}_{B} \int_{\mathbb{R}^{d}} \int_{\Omega}\left(\bigvee_{t \in M} \frac{B(t-z) V_{t}(\omega)}{c_{B} x_{t}}\right) \nu(\mathrm{d} \omega) \mathrm{d} z .
$$

a) If $X$ is stationary, then

$$
\int_{\Omega}\left(\bigvee_{t \in M} \frac{B(t-z) V_{t+h}(\omega)}{x_{t}}\right) \nu(\mathrm{d} \omega)=\int_{\Omega}\left(\bigvee_{t \in M} \frac{B(t-z) V_{t}(\omega)}{x_{t}}\right) \nu(\mathrm{d} \omega)
$$

for all $h \in \mathbb{R}^{d}$, all $z \in \mathbb{R}^{d}$ and all $B \in\{0,1\}^{\mathbb{R}^{d}}$. Therefore,

$$
\begin{aligned}
& \int_{\mathbb{R}^{d}} \int_{\Omega}\left(\bigvee_{t \in M} \frac{B((t+h)-z) V_{t+h}(\omega)}{x_{t}}\right) \nu(\mathrm{d} \omega) \mathrm{d} z \\
& =\int_{\mathbb{R}^{d}} \int_{\Omega}\left(\bigvee_{t \in M} \frac{B(t-z) V_{t}(\omega)}{x_{t}}\right) \nu(\mathrm{d} \omega) \mathrm{d} z
\end{aligned}
$$

for all $h \in \mathbb{R}^{d}$ and all integrable functions $B \in\{0,1\}^{\mathbb{R}^{d}}$.

b) The assertion follows from $(3.2)$ and the fact that $b_{1} v_{1} \vee b_{2} v_{2}=b_{1} b_{2}\left(v_{1} \vee v_{2}\right)$ for real numbers $b_{1}, b_{2}, v_{1}, v_{2}$ with $b_{i} \in\{0,1\}$ for $i=1,2$. 
Lemma 4.5.2. For all $1 \leq k \leq d$ The turning bands operator $\mathrm{TB}_{k}^{d}$ transfers members of the class $H_{1}$ into members of $H_{1}$.

Proof. The class $H_{1}$ is the class of continuous functions $h$ on $[0, \infty)$ that are convex and satisfy $h(0)=1$ and $\lim _{t \rightarrow \infty} h(t)=0$. All properties are preserved under $\mathrm{TB}_{k}^{d}$. For continuity and $\lim _{t \rightarrow \infty} h(t)=0$ use the dominated convergence theorem. Preservation of convexity follows from $\mathrm{TB}_{k}^{d}(h)(r)=\mathbb{E}_{A}(h(r c(A)))$ for $r \geq 0$ with $A \sim \sigma_{k}^{d}$ and $c(A)=\left\|A^{\mathrm{T}}(1,0, \ldots, 0)^{\mathrm{T}}\right\|_{2}$.

Proof of Proposition 4.4.9. A priori it is clear that $\varphi_{1}=h_{1}$ does not belong to $H_{k}$ for $k \geq 2$ [37.

a) Because of Proposition 4.4.4 the function $\varphi_{d}$ is a radial TCF on $\mathbb{R}^{d}$. Lemma 4.5.2 shows that $\varphi_{d}=\mathrm{TB}_{1}^{d}\left(h_{1}\right)$ belongs to $H_{1}$.

b) From 4.18, we compute that for $d \geq 2$

$$
-\varphi_{d}^{\prime}(\sqrt{t})=\beta_{d}\left\{\begin{array}{ll}
1 & t \leq 1 \\
1-(1-1 / t)^{(d-1) / 2} & t>1
\end{array},\right.
$$

where $\beta_{d}$ is the constant from 4.19 . Clearly, $-\varphi_{d}^{\prime}(\sqrt{t})$ is not convex. Therefore, one of the conditions of Theorem 3.1 in [37] (that is necessary to belong to the class $H_{3}$ ) is not fulfilled.

c) We verify that one of the conditions of Theorem 3.3 in [37] (that is necessary to belong to the class $\mathrm{H}_{2}$ ) is not fulfilled: Namely, we show that for all $d \geq 6$ the function

$$
c(t):=\int_{0}^{t} \sqrt{\frac{v}{t-v}}\left(-\varphi_{d}^{\prime}(1 / \sqrt{v})\right) \mathrm{d} v=\int_{0}^{1} \sqrt{\frac{w}{1-w}}\left(-\varphi_{d}^{\prime}(1 / \sqrt{t w}) \cdot t\right) \mathrm{d} w
$$

is not convex. From 4.22 we see that

$$
-\varphi_{d}^{\prime}(1 / \sqrt{v})=\beta_{d} \begin{cases}1-(1-v)^{(d-1) / 2} & v<1 \\ 1 & v \geq 1\end{cases}
$$


Since $d \geq 6$ we can compute the second derivative of $c$ at 1 :

$$
\begin{aligned}
c^{\prime \prime}(1) & =\left.\int_{0}^{1} \sqrt{\frac{w}{1-w}} \cdot \frac{\mathrm{d}^{2}}{\mathrm{~d} t^{2}}\right|_{t=1}\left(-\varphi_{d}^{\prime}(1 / \sqrt{t w}) \cdot t\right) \mathrm{d} w \\
& =\beta_{d}(d-1) \int_{0}^{1} w^{3 / 2}(1-w)^{(d-6) / 2}(1-w \cdot(d+1) / 4) \mathrm{d} w \\
& =\beta_{d}(d-1)\left(B\left(\frac{5}{2}, \frac{d-4}{2}\right)-\frac{d+1}{4} B\left(\frac{7}{2}, \frac{d-4}{2}\right)\right) \\
& =-\beta_{d}(d-1) \cdot \frac{3 \sqrt{\pi} \Gamma(d / 2-2)}{16 \Gamma((d+1) / 2)}<0
\end{aligned}
$$

Here $B(x, y)=\int_{0}^{1} t^{(x-1)}(1-t)^{y-1} \mathrm{~d} t$ denotes the Beta function. Since $c^{\prime \prime}(1)$ is negative, the function $c$ cannot be convex. This finishes the proof.

Lemma 4.5.3. If $f, g \in H_{1}$ then the product also belongs to this class $f \cdot g \in H_{1}$.

Proof. This is an immediate consequence of [37, Lemma 4.7] (or [100, Lemma 2]) which states that if $f$ and $g$ are non-negative, non-increasing and convex on an interval, then the product $f \cdot g$ is also non-negative, non-increasing and convex there.

Proof of Proposition 4.4.12, a) From Proposition 4.4.9 we know that $\varphi_{d}(2 t)$ is a radial TCF on $\mathbb{R}^{d}$ that belongs to $H_{1}$. Since $h_{d}(t)$ belongs to $H_{d}$ it follows from Example 4.4 .7 that the product $\chi_{d}(t)=\varphi_{d}(2 t) \cdot h_{d}(t)$ is a radial TCF on $\mathbb{R}^{d}$. Moreover $h_{d}(t)$ also belongs to $H_{d} \subset H_{1}$ and therefore $\chi_{d} \in H_{1}$ due to Lemma 4.5 .3

b) The function $\chi_{d}$ does not belong to the class of TCFs of variance-mixed BR processes on $\mathbb{R}^{d}$ because of its compact support (cf. the comment on region (D) in Figure 4.2.

c) It suffices to show that the function

$$
f(t):=-\chi_{3}^{\prime}(\sqrt{t})=-2 \varphi_{3}^{\prime}(\sqrt{4 t}) h_{3}(\sqrt{t})+\varphi_{3}(\sqrt{4 t})\left(-h_{3}^{\prime}(\sqrt{t})\right)
$$

is not convex, because then one of the conditions of Theorem 3.1 in [37] (that is necessary to belong to the class $H_{3}$ ) is not fulfilled. From 4.6, 4.18) and 4.22 we see that for $t \in[0,1]$

$$
\begin{aligned}
h_{3}(\sqrt{t}) & =\frac{1}{2}\left(2-3 t^{1 / 2}+t^{3 / 2}\right), & -h_{3}^{\prime}(\sqrt{t}) & =\frac{3}{2}(1-t), \\
\varphi_{3}(\sqrt{4 t}) & =\left\{\begin{array}{lll}
1-\sqrt{t} & t \leq 1 / 4 \\
1 /(4 \sqrt{t}) & t \geq 1 / 4
\end{array},\right. & -2 \varphi_{3}^{\prime}(\sqrt{4 t}) & =\left\{\begin{array}{ll}
1 & t \leq 1 / 4 \\
1 /(4 t) & t \geq 1 / 4
\end{array} .\right.
\end{aligned}
$$


Thus, $f(t)$ is a decreasing function on $[0,1]$ with the following left-hand and right-hand derivative at $1 / 4$

$$
\lim _{t \uparrow 1 / 4} f^{\prime}(t)=-3 \quad \text { and } \quad \lim _{t \downarrow 1 / 4} f^{\prime}(t)=-17 / 4 \text {. }
$$

Hence, $f$ cannot be convex in a neighborhood of $1 / 4$.

\subsubsection{Derivation of expressions in tables}

Proof of Table 4.1. The TCF of a Brown-Resnick process has been computed in [52. Remark 25]. If $X$ is a variance-mixed BR process and $V_{t}=\exp \left(S W_{t}-\frac{S^{2}}{2} \sigma^{2}(t)\right)$ as in Example 1.2.6, then conditioning on $S$ yields the TCF of $X$

$$
\begin{aligned}
\chi_{\mathrm{VBR}}(t) & =\mathbb{E}\left(V_{t} \wedge V_{o}\right)=\mathbb{E}\left(\mathbb{E}\left(V_{t} \wedge V_{o} \mid S\right)\right) \\
& =\mathbb{E} \operatorname{erfc}\left(\sqrt{S^{2} \gamma(t) / 8}\right)=\int_{0}^{\infty} \operatorname{erfc}\left(s \sqrt{\frac{\gamma(t)}{8}}\right) \mathrm{d} G(s) .
\end{aligned}
$$

For extremal Gaussian processes we refer to [11, (7)] and (3.6). Now, let $X$ be an extremal binary Gaussian process and let $Z$ be a Gaussian process with correlation function $\rho$ as in Example 1.2.2 Then [12, Equation (10.8.3)] gives (with $u=0$ therein)

$$
\begin{aligned}
\chi_{\mathrm{EBG}}(t) & =\mathbb{P}\left(Z_{t}>0 \mid Z_{o}>0\right)=2 \mathbb{P}\left(Z_{t}>0, Z_{o}>0\right) \\
& =2\left(\frac{1}{2 \pi} \arcsin (\rho(t))+\frac{1}{4}\right)=\frac{1}{\pi} \arcsin (\rho(t))+\frac{1}{2} .
\end{aligned}
$$

The TCF of an M3 processes has been considered before in (3.2.2). Because of 2.4 and (3.6) the TCF of a Mixed Poisson storm process is given by

$$
\chi_{\mathrm{MPS}}(t)=\mathcal{L}(F)(b([o, t]))=\mathcal{L}(F)\left(\frac{2 \kappa_{d-1}}{d \kappa_{d}}\|t\|_{2}\right),
$$

where $b([o, t])$ denotes the mean width of the line segment $[o, t] \subset \mathbb{R}^{d}$ between the origin $o$ and $t \in \mathbb{R}^{d}$ (cf. [88, p. 601 (14.7)]).

Lemma 4.5.4. Let $d \geq 3$ be odd and $\varphi \in H_{d}=M_{d}^{o}$. Let $G$ be a corresponding distribution function as in (4.5) in the definition of the class $H_{d}$ and let $f$ be a nonincreasing shape function as in the definition of the class $M_{d}^{o}$. Set $k:=(n-1) / 2$ 
and define the (right-hand) derivative

$$
\lambda(t):=(-1)^{k} \frac{\mathrm{d}^{k}}{\mathrm{~d} t^{k}}\left[-\varphi^{\prime}(\sqrt{t})\right] \quad t \geq 0
$$

Then $G$ and $f$ can be recovered from $\varphi$ by

$$
G(s)=\frac{\sqrt{\pi}}{d \Gamma(d / 2)} \int_{0}^{s} \frac{1}{t^{d}} \mathrm{~d} \lambda\left(\frac{1}{t^{2}}\right) \quad \text { and } \quad f(u)=\left(\frac{2}{\sqrt{\pi}}\right)^{d-1} \lambda\left(4 u^{2}\right) .
$$

Proof. The recovery of $G$ is precisely [37, Theorem 3.2]. By 4.8 we obtain

$$
\begin{aligned}
f(u) & =\frac{1}{b_{d}} \int_{0}^{1 /(2 u)} s^{d} \mathrm{~d} G(s)=\frac{\sqrt{\pi}}{b_{d} d \Gamma(d / 2)} \int_{0}^{1 /(2 u)} \mathrm{d} \lambda\left(\frac{1}{s^{2}}\right) \\
& =\left(\frac{2}{\sqrt{\pi}}\right)^{1 /(2 u)}\left(\lambda\left(4 u^{2}\right)-\lim _{x \rightarrow \infty} \lambda(x)\right)
\end{aligned}
$$

But $\lim _{x \rightarrow \infty} \lambda(x)$ necessarily vanishes, since $\lambda(t)=-a^{\prime}(t)$ for a non-negative (i.e. bounded from below), non-increasing and convex function $a(t)$ due to [37, (22)].

Proof of Table 4.2. In case $d=1$ we refer to [37, (18)] for the recovery of $G$ and $g=G^{\prime}$. The recovery of $f$ follows from 4.21. In case $d=3$ the previous Lemma 4.5 .4 can be applied to $d=3$ and $\varphi=\chi$, where we abbreviate $\lambda_{\chi}(t)=2 \lambda\left(1 / t^{2}\right)=$ $t \chi^{\prime \prime}(1 / t)$. In case $d=2$ we additionally assume that $\chi \in H_{5}$, such that

$$
(-1)^{k} \frac{\mathrm{d}^{k}}{\mathrm{~d} t^{k}}\left[-\chi^{\prime}(\sqrt{t})\right]
$$

exists for $k \in\{0,1,2\}$ and is non-negative, non-increasing and convex for $k \in\{0,1\}$ (cf. [37, p. 96]). This requirement ensures that we can apply the monotone convergence theorem iteratively when differentiating within the following integral (4.24). A priori we know from [37, Theorem 3.4] that

$$
G(r)=\frac{1}{2} \int_{(0, r)} \frac{1}{s} \mathrm{~d} \mu\left(s^{2}\right) \quad \text { with } \quad \mu(t)=\frac{\mathrm{d}}{\mathrm{d} t} \int_{0}^{t} \sqrt{\frac{v}{t-v}}\left[-\chi^{\prime}(1 / \sqrt{v})\right] \mathrm{d} v .
$$

Now $\chi \in H_{5}$ ensures that $\mu^{\prime}(t)$ exists by

$$
\begin{aligned}
\mu^{\prime}(t) & =\frac{\mathrm{d}^{2}}{\mathrm{~d} t^{2}} \int_{0}^{t} \sqrt{\frac{v}{t-v}}\left[-\chi^{\prime}(1 / \sqrt{v})\right] \mathrm{d} v=\frac{\mathrm{d}^{2}}{\mathrm{~d} t^{2}}\left(t \int_{0}^{1} \sqrt{\frac{w}{1-w}}\left[-\chi^{\prime}(1 / \sqrt{w t})\right] \mathrm{d} w\right) \\
& =\int_{0}^{1} \sqrt{\frac{w}{1-w}}\left(\frac{\mathrm{d}^{2}}{\mathrm{~d} t^{2}}\left[-t \chi^{\prime}(1 / \sqrt{w})\right]\right) \mathrm{d} w
\end{aligned}
$$


where

$$
\frac{\mathrm{d}^{2}}{\mathrm{~d} t^{2}}\left[-t \chi^{\prime}(1 / \sqrt{w t})\right]=\frac{1}{4 t \sqrt{w t}}\left[\chi^{\prime \prime}\left(\frac{1}{\sqrt{w t}}\right)-\frac{1}{\sqrt{w t}} \chi^{\prime \prime \prime}\left(\frac{1}{\sqrt{w t}}\right)\right] .
$$

The substitutions $v=w t$ and $v=u^{2}$ give

$$
\mu^{\prime}(t)=\frac{1}{2 t^{2}} \int_{0}^{\sqrt{t}} \sqrt{\frac{u^{2}}{t-u^{2}}} \mathrm{~d} \lambda_{\chi}(u) \quad \text { with } \quad \lambda_{\chi}(u)=u \chi^{\prime \prime}(1 / u) .
$$

Hence $G$ has a density $g$ with

$$
g(s)=\mu^{\prime}\left(s^{2}\right)=\frac{1}{2 s^{4}} \int_{0}^{s} \sqrt{\frac{1}{(s / u)^{2}-1}} \mathrm{~d} \lambda_{\chi}(u) .
$$

Fubini's theorem and the substitution $s=1 / t$ yield

$$
\begin{aligned}
& G(r)=\int_{0}^{r} g(s) \mathrm{d} s=\int_{0}^{r} \frac{1}{2 s^{4}} \int_{0}^{s} \sqrt{\frac{1}{(s / u)^{2}-1}} \mathrm{~d} \lambda_{\chi}(u) \mathrm{d} s \\
& =\frac{1}{2} \int_{0}^{r}\left(\int_{u}^{r} \frac{1}{s^{4}} \frac{1}{\sqrt{s^{2}-u^{2}}} \mathrm{~d} s\right) u \mathrm{~d} \lambda_{\chi}(u)=\frac{1}{2} \int_{0}^{r}\left(\int_{1 / r}^{1 / u} \frac{t^{3}}{\sqrt{1-t^{2} u^{2}}} \mathrm{~d} t\right) u \mathrm{~d} \lambda_{\chi}(u) .
\end{aligned}
$$

Applying [42, p. 96 2.264.4] we arrive at

$$
\begin{aligned}
G(r) & =\frac{1}{2} \int_{0}^{r}\left(\frac{1}{3 u^{2} r^{2}}+\frac{2}{3 u^{4}}\right)\left(\sqrt{1-\frac{u^{2}}{r^{2}}}\right) u \mathrm{~d} \lambda_{\chi}(u) \\
& =\frac{1}{6 r^{3}} \int_{0}^{r}\left(1+2\left(\frac{r}{u}\right)^{2}\right)\left(\sqrt{\left(\frac{r}{u}\right)^{2}-1}\right) \mathrm{d} \lambda_{\chi}(u) .
\end{aligned}
$$

To compute the shape function $f$ we apply 4.8

$$
\frac{\pi}{4} f\left(\frac{1}{2 u}\right)=\int_{0}^{u} s^{2} g(s) \mathrm{d} s=\frac{1}{2} \int_{0}^{u} \frac{1}{s^{2}} \int_{0}^{s} \sqrt{\frac{1}{(s / t)^{2}-1}} \mathrm{~d} \lambda_{\chi}(t) \mathrm{d} s .
$$

By Fubini's theorem and the substitution $s=1 / r$ we have

$$
\begin{aligned}
\frac{\pi}{4} f\left(\frac{1}{2 u}\right) & =\frac{1}{2} \int_{0}^{u}\left(\int_{t}^{u} \frac{1}{s^{2}} \frac{1}{\sqrt{s^{2}-t^{2}}} \mathrm{~d} s\right) t \mathrm{~d} \lambda_{\chi}(t) \\
& =\frac{1}{2} \int_{0}^{u}\left(\int_{1 / u}^{1 / t} \frac{r}{\sqrt{1-r^{2} t^{2}}} \mathrm{~d} r\right) t \mathrm{~d} \lambda_{\chi}(t) .
\end{aligned}
$$


Applying [42, p. 96 2.264.2] gives

$$
\frac{\pi}{4} f\left(\frac{1}{2 u}\right)=\frac{1}{2} \int_{0}^{u}\left(\frac{\sqrt{u^{2}-t^{2}}}{u t^{2}}\right) t \mathrm{~d} \lambda_{\chi}(t)=\frac{1}{2 u} \int_{0}^{u}\left(\sqrt{\left(\frac{u}{t}\right)^{2}-1}\right) \mathrm{d} \lambda_{\chi}(t) .
$$

Finally, we replace $u$ by $1 /(2 u)$ and obtain

$$
f(u)=\frac{4 u}{\pi} \int_{0}^{1 /(2 u)}\left(\sqrt{\left(\frac{1}{2 u t}\right)^{2}-1}\right) \mathrm{d} \lambda_{\chi}(t)
$$

as desired.

Lemma 4.5.5. Let $g(s)=\sqrt{\pi} f\left(s^{2}\right)$ be a probability density on $(0, \infty)$ and let $\varphi:[0, \infty) \rightarrow[0,1]$ with $\varphi(0)=1$ be such that $-\varphi^{\prime}(\sqrt{\cdot})$ is the Laplace transform of $f$ in the following sense

$$
-\varphi^{\prime}(\sqrt{t})=\int_{0}^{\infty} e^{-r t} f(r) \mathrm{d} r
$$

Then

$$
\varphi(t)=\int_{0}^{\infty} \operatorname{erfc}(s t) g(s) \mathrm{d} s .
$$

Proof. (analogously to [37, p. 104]) Replacing $t$ by $t^{2}$ and $r$ by $s^{2}$ yields

$$
-\varphi^{\prime}(t)=\int_{0}^{\infty} 2 s e^{-s^{2} t^{2}} f\left(s^{2}\right) \mathrm{d} s=\int_{0}^{\infty} \frac{\mathrm{d}}{\mathrm{d} t}[-\operatorname{erfc}(s t)] g(s) \mathrm{d} s .
$$

Applying Fubini's theorem when integrating w.r.t. $t$ gives

$$
\varphi(0)-\varphi(t)=\int_{0}^{\infty}[\operatorname{erfc}(0)-\operatorname{erfc}(s t)] g(s) \mathrm{d} s,
$$

which entails the claim, since $g$ is a density on $(0, \infty)$ and $\varphi(0)=1$.

Proof of Table 4.3. We apply Lemma 4.5.5 and derive this table from known Laplace transforms in [77] using (in this order) equations [p. 9645.3 (11)], [p. 9645.3 (12), p. 9635.2 (12) and p. 9625.1 (26)], [p. 9635.3 (1)] and [p. 9635.3 . (3) with $\nu=1.5$ ] therein. 


\subsubsection{Plots}
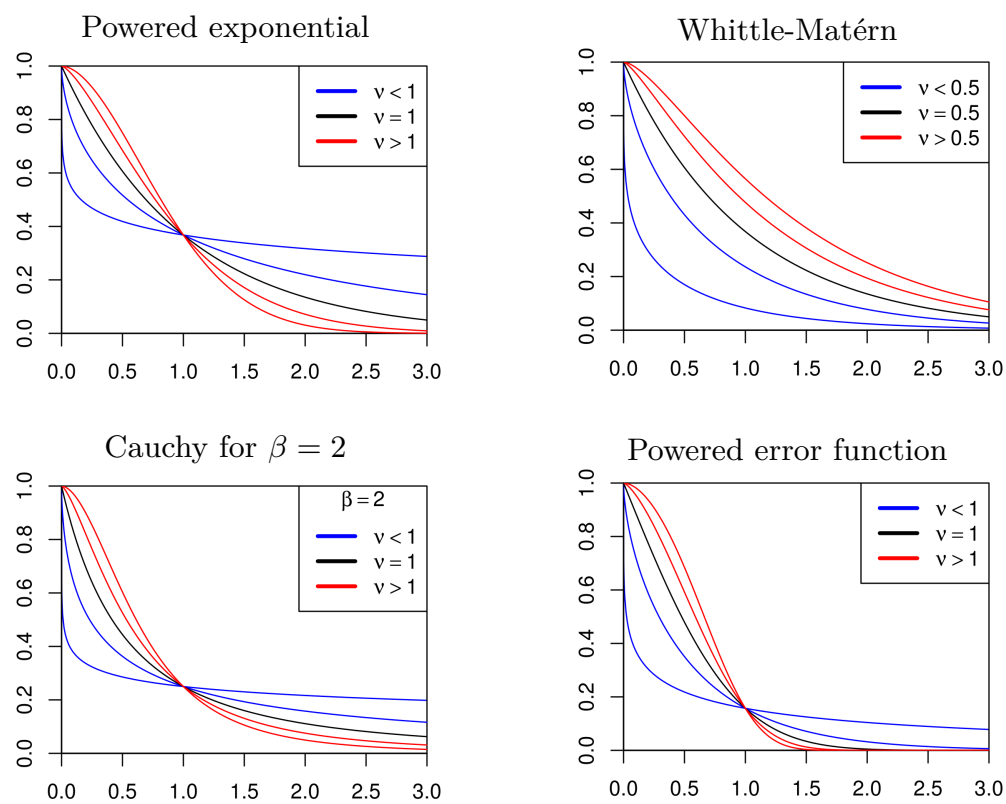

Figure 4.3.: Parametric families of radially symmetric functions on $\mathbb{R}^{d}$ for the parameters $\nu=0.2,0.6,1,1.4,1.8$ (powered exponential, Cauchy, powered error function) and $\nu=0.1,0.3,0.5,0.7,0.9$ (Whittle-Matérn). The sharp parameter bound for being a tail correlation function (TCF) is marked black, blue functions are TCFs, red functions not, see Table 4.4 

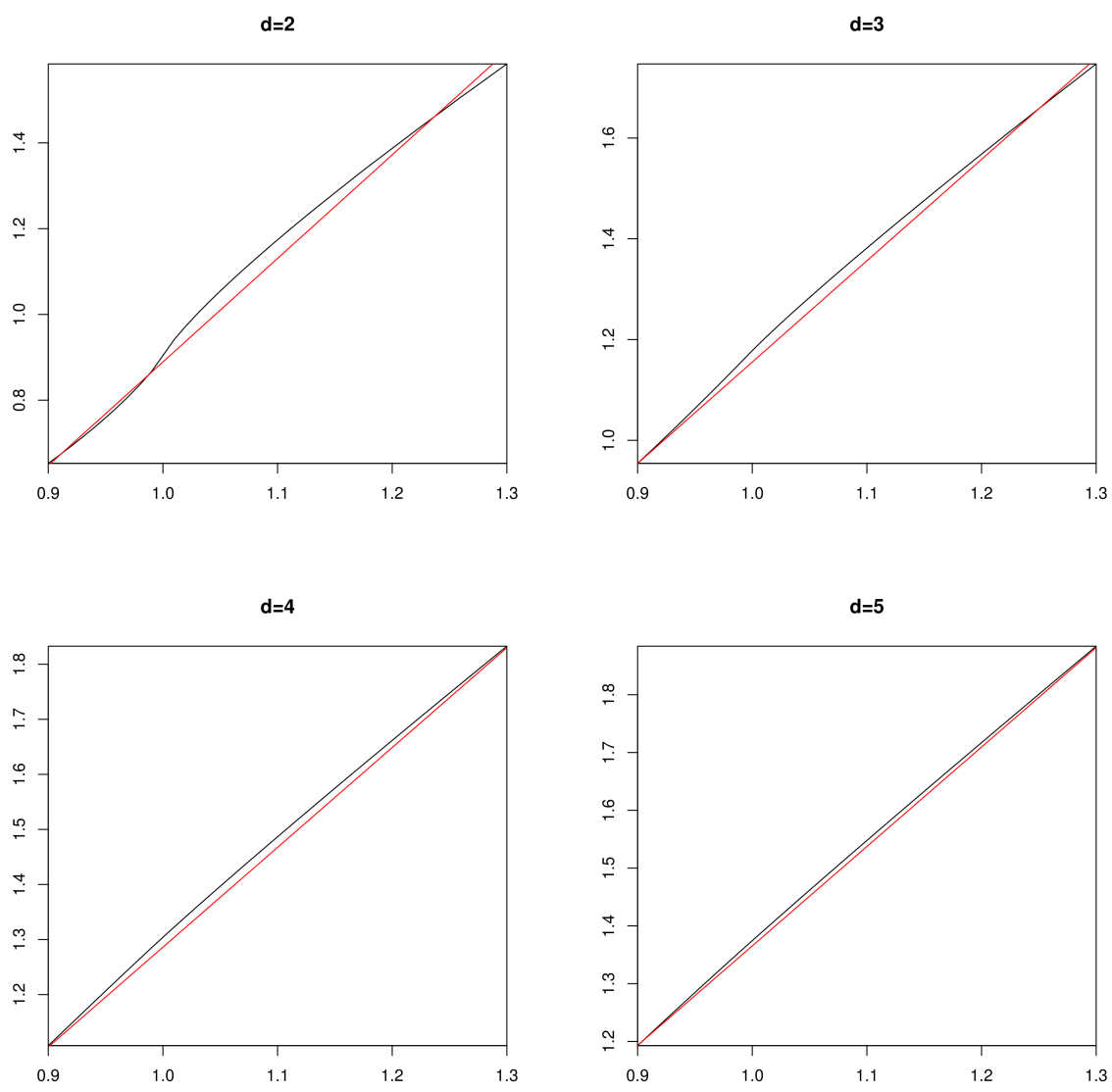

Figure 4.4.: The function $c(t) / \beta_{d}$ with $c(t)$ from $(4.23)$ and $\beta_{d}$ as in 4.19 is plotted for $d \in\{2,3,4,5\}$ (black line). Additionally a straight line (red line) indicates that the respective functions are not convex (cf. Remark 4.4.10). 
(John Lennon)

\section{Representations of Brown-Resnick processes for group actions}

In [52] Brown-Resnick processes are introduced as stationary processes on $\mathbb{R}^{d}$. Moreover, a condition is derived that guarantees that such a process can be represented as a Mixed Moving Maxima process (cf. [52, Theorem 14]). Here, instead of stationarity on $\mathbb{R}^{d}$, we include other types of invariance, when a group action on some space $T$ is considered. As a convention we will consider left group actions.

Definition 5.0.6. Let $G$ be a group with composition $(g, h) \mapsto g h$ and identity element $e$. Let $T$ be a set. Then we call a map $G \times T \rightarrow T$ which we write as $(g, t) \rightarrow g t$ a (left) group action if $g(h t)=(g h) t$ and $e t=t$ for all $g, h \in G$ and $t \in T$. In that case we say that $G$ acts on $T$ (from the left). The orbit of an element $t \in T$ is the set

$$
G t:=\{g t: g \in G\}
$$

The action is said to be transitive if there is only one orbit, i.e. $T=G t$ for some (and then all) $t \in T$. The stabilizer (subgroup) of an element $t \in T$ is

$$
\operatorname{Stab}(t):=\{g \in G: g t=t\} \subset G .
$$

The action is said to be free if $\operatorname{Stab}(t)=\{e\}$ for all $t \in T$. Elements $t \in T$ with $\operatorname{Stab}(t)=G$ are called fixed points. If $G$ and $T$ are topological spaces, the action is said to be continuous if the respective map $G \times T \rightarrow T$ is continuous.

Example 5.0.7. As an example we have in mind the 2-sphere $S^{2}$ (the unit sphere in $\mathbb{R}^{3}$ ) and the rotating $S^{1}$-action on $S^{2}$ around some fixed axis. This group action is continuous and has two fixed points $n$ and $s$. The group $S^{1}$ acts freely on $S^{2} \backslash\{n, s\}$. 
Definition 5.0.8. Let $G$ be a (left) group action on some space $T$. We say that a stochastic process $X$ on $T$ is $G$-invariant (w.r.t. to this group action) if the law of $\left\{X_{g t}\right\}_{t \in T}$ and the law of $X$ coincide for every $g \in G$.

In the sequel we shall derive a Mixed Moving Maxima representation for $G$ invariant Brown-Resnick processes (to be introduced below) when a suitable group action of some compact group is considered. The difficulty here does not lie in transferring the proof of [52] to the new situation, but in establishing a measurability result. In fact, this measurability problem does not occur in the previous case, since the question of a unique measurable argmax can be avoided by taking the (lexicographic) infimum which commutes with the translation action of $\mathbb{R}^{d}$ on $\mathbb{R}^{d}$.

In order to stress the parallels to previous work, we consider again Brown-Resnick processes with standard Gumbel marginals (and not standard Fréchet marginals). We write $\eta(t)$ (and not $\eta_{t}$ ) to emphasize the continuous paths later on. To be more precise, let $(\Omega, \mathcal{A}, \nu, V)$ be a spectral representation of a simple max-stable process $X$ on $T$ such that

$$
X_{t}=\bigvee_{n=1}^{\infty} U_{n} V_{t}\left(\omega_{n}\right) \quad t \in T
$$

where $\left\{\left(U_{n}, \omega_{n}\right)\right\}_{n=1}^{\infty}$ denotes a Poisson point process on $\mathbb{R}_{+} \times \Omega$ with intensity $u^{-2} \mathrm{~d} u \nu(\mathrm{d} \omega)$. Further, assume that $(\Omega, \mathcal{A}, \nu)$ is a probability space and

$$
V_{t}(\omega)=\exp \left(\xi_{\omega}(t)\right) \quad(\omega, t) \in \Omega \times T
$$

for a stochastic process $\xi$ on $T$, which is distributed according to $\nu$. Then we will consider, instead of $X$ as above, the max-stable process $\eta=\{\eta(t)\}_{t \in T}$ with

$$
\eta(t)=\bigvee_{n=1}^{\infty} U_{n}+\xi_{n}(t) \quad t \in T
$$

where $U_{n}$ denotes a Poisson point process on $\mathbb{R}$ with intensity $e^{-u} \mathrm{~d} u$ and $\left(\xi_{n}\right)_{n=1}^{\infty}$ an independent sequence of i.i.d. processes on $T$ distributed according to $\nu$. In what follows we omit the measure $\nu$ and write $\mathbb{P}_{\xi}$ instead or $\mathbb{E}_{\xi}$ when referring to the law and expectation of $\xi$ respectively. Because of our standing normalization requirement

$$
\mathbb{E}_{\xi}\left(e^{\xi(t)}\right)=\int_{\Omega} V_{t}(\omega) \mathrm{d} \nu=1
$$

the process $\eta$ has standard Gumbel marginals. Note that the law of the process $X$ equals the law of $e^{\eta}$. 


\subsection{Brown-Resnick processes for group actions}

The proof of [52, Proposition 6] which is carried out for the translation action of $\mathbb{R}^{d}$ on $\mathbb{R}^{d}$ transfers verbatim to the following situation (cf. also Proposition A.3.1.

Proposition 5.1.1. Let $\eta=\{\eta(t)\}_{t \in T}$ be a max-stable process with standard Gumbel marginals as in (5.1). Then $\eta$ is $G$-invariant if and only if

$$
\mathbb{E}\left(\exp \left(u_{1} \xi_{t_{1}}+u_{2} \xi_{t_{2}}+\cdots+u_{n} \xi_{t_{n}}\right)\right)=\mathbb{E}\left(\exp \left(u_{1} \xi_{g t_{1}}+u_{2} \xi_{g t_{2}}+\cdots+u_{n} \xi_{g t_{n}}\right)\right)
$$

for all $g \in G$ and $t_{1}, \ldots, t_{n} \in \mathbb{R}^{d}$ and $u_{1}, \ldots, u_{n} \in[0,1]$ with $\sum_{i=1}^{n} u_{i}=1$.

Moreover, [52, Theorem 2] admits the following version, when the respective proof is adapted or [49, Theorem 1] is applied (cf. also Theorem A.3.2). For convenience, we will only consider zero mean Gaussian processes.

Theorem 5.1.2. Let $\{W(t)\}_{t \in T}$ be a zero mean Gaussian process with variance $\sigma^{2}(t)=\operatorname{Var}(W(t))$ and variogram $\gamma(s, t)=\mathbb{E}(W(s)-W(t))^{2}$, such that

$$
\gamma(g s, g t)=\gamma(s, t) \quad \forall g \in G, \forall s, t \in T \text {. }
$$

Set $\xi(t):=W(t)-\sigma^{2}(t) / 2$ for $t \in T$. Then $\mathbb{E} e^{\xi}(t)=1$ and thus, $\{\xi(t)\}_{t \in T}$ defines a max-stable process $\eta=\{\eta(t)\}_{t \in T}$ with standard Gumbel marginals via (5.1). This process $\eta$ has the following properties.

a) $\eta$ is G-invariant.

b) The law of $\eta$ depends only on the variogram $\gamma$.

As in the case of stationary Brown-Resnick processes on $\mathbb{R}^{d}$, such a process $\eta$ as in Theorem 5.1.2 will be also be referred to as Brown-Resnick process (w.r.t. the $G$-action).

Remark 5.1.3. (cf. [52, Remark 12]) It is convenient to fix some $t_{o} \in T$ and to consider the process $\widetilde{W}(t)=W(t)-W\left(t_{o}\right)=0$ instead of $W$, which is also Gaussian, has the same variogram and satisfies $\widetilde{W}\left(t_{o}\right)=0$. Its variance is $\widetilde{\sigma}^{2}(t)=\operatorname{Var}(\widetilde{W}(t))=\gamma\left(t, t_{o}\right)$.

Remark 5.1.4. As in Example 3.2.3 the TCF of the Brown-Resnick process $\eta$ from Theorem 5.1.2 is given by $\chi(s, t)=\operatorname{erfc}(\sqrt{\gamma(s, t) / 8})$.

Remark 5.1.5. Let $\{W(t)\}_{t \in T}$ be a zero mean Gaussian process with variogram $\gamma(s, t)=\mathbb{E}(W(s)-W(t))^{2}$ and consider additionally to 5.2 the following statements. 
(i) The process $\{W(s)-W(t)\}_{(s, t) \in T \times T}$ is $G$-invariant for the $G$-action $g(s, t)=(g s, g t)$ on $T \times T$.

(ii) The process $\{W(g t)-W(t)\}_{t \in T}$ is $G$-invariant for all $g \in G$.

(iii) The expression $\gamma(g t, t)$ does not depend on $t \in T$ for all $g \in G$.

(iv) The law of the process $\{W(g t)-W(t)\}_{g \in G}$ does not depend on $t \in T$.

Then the statements (5.2) and (ii) are equivalent and secondly, the statements (iii) and (iv) are equivalent. If $G$ is abelian, (i) implies (ii). If additionally $G$ acts transitively on $T$, then all five statements are equivalent. To verify these implications take into account that

$$
2 \operatorname{Cov}\left(W(s)-W(t), W\left(s^{\prime}\right)-W\left(t^{\prime}\right)\right)=\gamma\left(s, t^{\prime}\right)+\gamma\left(t, s^{\prime}\right)-\gamma\left(s, s^{\prime}\right)-\gamma\left(t, t^{\prime}\right) .
$$

An example of a transitive action of an abelian group is, of course, the translation action of $G=\mathbb{R}^{d}$ on $T=\mathbb{R}^{d}$. Here, (ii) means that $W$ is intrinsically stationary and (iv) means that $W$ has stationary increments. Other cases require more caution. Consider the rotating $S^{1}$-action on the 2 -sphere $S^{2} \subset \mathbb{R}^{3}$ around some fixed axis, which has two fixed points. Then (iii) implies immediately $\gamma(s, t)=0$ if $s$ and $t$ belong to the same orbit, whereas more interesting examples for (5.2) exist. For instance, the angular distance between $s$ and $t$ is a valid variogram (cf. [43]) and clearly invariant w.r.t. rotations.

\subsection{Mixed Moving Maxima for group actions}

Let us modify the definition of an M3 process as in Example 1.2.1 from the translation action on $\mathbb{R}^{d}$ to certain $G$-actions on a space $T$. To this end, we use the notion of Haar measures as treated in [68, for instance. Recall that, in contrast to Example 1.2.1. we work here with standard Gumbel marginals.

Definition 5.2.1. Let $G$ be a locally compact topological group acting continuously (from the left) on a topological space $T$. Denote by $\mu$ a (left) Haar measure on $G$. If $G$ is compact, we make our choice of Haar measure unique by $\mu(G)=1$.

Let $\kappa=\{\kappa(t)\}_{t \in T}$ be a measurable process on $T$ such that

$$
\mathbb{E}_{\kappa}\left(\int_{G} e^{\kappa\left(g^{-1} t\right)} \mu(\mathrm{d} g)\right)=1 \quad \forall t \in T
$$


Let $\left(\kappa_{n}\right)_{n=1}^{\infty}$ be i.i.d. copies of $\kappa$ and let $\left(g_{n}, U_{n}\right)_{n=1}^{\infty}$ be an independent Poisson point process on $G \times \mathbb{R}$ with intensity $e^{-u} \mu(\mathrm{d} g) \mathrm{d} u$. We call a process of the form

$$
\eta(t):=\bigvee_{n=1}^{\infty}\left(U_{n}+\kappa_{n}\left(g_{n}^{-1} t\right)\right) \quad t \in T
$$

Mixed Moving Maxima process (M3 process) w.r.t. the G-action.

For a non-empty finite set $M \subset T$ the f.d.d. of $\eta$ in Definition 5.2.1 are given by

$$
-\log \mathbb{P}\left(\eta(t) \leq y_{t}, t \in M\right)=\mathbb{E}_{\kappa} \int_{G} \exp \left(\bigvee_{t \in M} \kappa\left(g^{-1} t\right)-y_{t}\right) \mu(\mathrm{d} g)
$$

for $y \in \mathbb{R}^{M}$. In particular $\eta$ is max-stable with standard Gumbel marginals. To see the $G$-invariance of the f.d.d. of $\eta$, fix some $y \in \mathbb{R}^{m}$ and locations $t_{1}, \ldots, t_{m}$ and note that the function $\tilde{\kappa}_{t_{1}, \ldots, t_{m}}(g):=\mathbb{E}_{\kappa} \exp \left(\bigvee_{t \in M}\left(\kappa\left(g^{-1} t_{i}\right)-y_{i}\right)\right)$ on $G$ is integrable. Since $\mu$ is a (left) Haar measure, we have for any $h \in G$

$$
\int_{G} \tilde{\kappa}_{h t_{1}, \ldots, h t_{m}}(g) \mu(\mathrm{d} g)=\int_{G} \tilde{\kappa}_{t_{1}, \ldots, t_{m}}\left(h^{-1} g\right) \mu(\mathrm{d} g)=\int_{G} \tilde{\kappa}_{t_{1}, \ldots, t_{m}}(g) \mu(\mathrm{d} g),
$$

which shows $\mathbb{P}\left(\eta\left(h t_{i}\right) \leq y_{i}, i=1, \ldots, m\right)=\mathbb{P}\left(\eta\left(t_{i}\right) \leq y_{i}, i=1, \ldots, m\right)$ for all $h \in G$.

\subsection{M3 representation for actions of compact groups}

The following theorem is the main result of this chapter. It can be viewed as an analogous version of [52, Theorem 14] for compact groups.

Theorem 5.3.1. Let $G$ be a compact metric group acting continuously on a $\sigma$ locally-compact metric space $T$, such that there exists $t_{o}$ with

$$
\operatorname{Stab}\left(t_{o}\right) \subset \operatorname{Stab}(t) \quad \forall t \in T \text {. }
$$

Let $\{W(t)\}_{t \in T}$ be a sample-continuous zero mean Gaussian process with variance $\sigma^{2}(t)=\operatorname{Var}(W(t))$ and variogram $\gamma(s, t)=\mathbb{E}(W(s)-W(t))^{2}$, such that

$$
\begin{aligned}
\gamma(g s, g t) & =\gamma(s, t) & & \forall s, t \in T, \forall g \in G, \\
\gamma(s, t) & \neq 0 & & \forall s, t \in T \text { with } s \neq t .
\end{aligned}
$$

Set $\xi(t):=W(t)-\sigma^{2}(t) / 2$ and let $\eta$ be the Brown-Resnick process from Theorem 5.1.2. Then there exists a Mixed Moving Maxima process $\eta^{*}$ that has the same law as $\eta$. 
Example 5.3.2. The requirement that there exists $t_{o}$ with $\operatorname{Stab}\left(t_{o}\right) \subset \operatorname{Stab}(t)$ for all $t \in T$ will be met if all stabilizer subgroups $\operatorname{Stab}(t) \subset G$ for $t \in T$ coincide. For instance, there is an $S^{3}$ action on $\mathrm{SO}(3)$ with stabilizers $\{ \pm 1\}$. The following scenarios are also included in this situation:

(a) $G$ acts freely on $T$, e.g. $S^{1}$ acts freely on $\mathbb{R}^{2} \backslash\{o\}$ by rotation around the origin.

(a.1) Each group $G$ acts freely on itself by left multiplication. For instance, the 3 -sphere $S^{3} \subset \mathbb{R}^{4}$ admits a group action and acts freely on itself.

(b) $G$ acts transitively on $T$ and the stablilizer subgroup $\operatorname{Stab}\left(t_{o}\right) \subset G$ of some $t_{o} \in$ $T$ (hence every $t_{o} \in T$ ) is a normal subgroup of $G$ (meaning $g \operatorname{Stab}\left(t_{o}\right) g^{-1}=$ $\operatorname{Stab}\left(t_{o}\right)$ for $\left.g \in G\right)$. In this case $T \cong G / \operatorname{Stab}\left(t_{o}\right)$ is necessarily compact (see Lemma 5.3.4.

(b.1) This is includes transitive actions of abelian groups, e.g. $S^{1} \times S^{1}$ acts transitively on the 2-dimensional torus.

On the other hand, it is not necessary, to require that all stabilizer subgroups coincide. For instance, in addition to the above scenarios fixed points $t_{*}$ (with $\operatorname{Stab}\left(t_{*}\right)=G$ ) can be admitted as in the rotating $S^{1}$-action on $\mathbb{R}^{2}$ or on the 2 -sphere $S^{2}$. However, 5.4 does not include the case that the stabilizer subgroups are only conjugate. The isometric $\mathrm{SO}(3)$-action on $S^{2}$ may not be considered, for example.

Example 5.3.3. Consider again the rotating $S^{1}$-action on the sphere $S^{2}$, which has two fixed points. This example meets (5.4). Many suitable variograms for Theorem 5.3.1 that depend only on the spherical distance can be found in [43.

The following lemma summarizes some facts concerning the group action involved in Theorem 5.3.1. We refer to [73] and [72. A detailed study of Haar measures on locally compact homogeneous spaces can be found in 68 .

Lemma 5.3.4. Let $G$ be a compact metric group acting continuously on a $\sigma$-locallycompact metric space $T$ (from the left). Let $t_{o} \in T$ and $G t_{o} \subset T$ its (compact) orbit and $\operatorname{Stab}\left(t_{o}\right) \subset G$ the stabilizer of $t_{o}$, which is a closed (hence compact) subgroup of $G$. Then the map

$$
G / \operatorname{Stab}\left(t_{o}\right) \rightarrow G t_{o}, \quad[g] \rightarrow g t_{o}
$$

is well-defined and a homeomorphism. In particular $H_{o}:=G / \operatorname{Stab}\left(t_{o}\right) \cong G t_{o}$ is compact and locally compact. The group $G$ acts continuously on $H_{o}$ by left multiplication: $g[h]:=[g h]$. 
If additionally $\operatorname{Stab}\left(t_{o}\right)$ is a normal subgroup in $G$, then $H_{o}$ is also a compact metric group and the continuous quotient map $G \rightarrow H_{o}$ where $g \mapsto[g]$ is a group homomorphism. Any finite $G$-invariant (or equivalently $H_{o}$-invariant) measure on the Borel- $\sigma$-algebra of $H_{O}$ is a constant multiple of the (left) Haar measure $\tilde{\mu}$ on $H_{o}$ with $\tilde{\mu}\left(H_{o}\right)=1$. The (probability) Haar measure $\tilde{\mu}$ on $H_{o}$ is the pushforward measure of the (probability) Haar measure $\mu$ on $G$ under the quotient map $G \rightarrow H_{o}$.

Before we can prove Theorem 5.3.1, we need to establish a crucial preparatory result, which is also of independent interest.

Lemma 5.3.5. Let $(T, d)$ be a compact metric space and $(C(T),\|\cdot\|)$ the Banach space of real-valued continuous functions on $T$ endowed with the supremum norm $\|\cdot\|$. Let $C_{\operatorname{amax}}(T)$ be the subset of functions $f \in C(T)$, such that there exists a unique point $t \in T$, at which the maximum value of $f$ is attained. Then $C_{\text {amax }}(T)$ is measurable w.r.t. the Borel- $\sigma$-algebra of the supremum norm and the map

$$
\operatorname{argmax}: C_{\text {amax }}(T) \rightarrow T,
$$

which assigns to each function $f \in C_{\mathrm{amax}}(T)$ this unique point, is well-defined and measurable.

Proof. For $f \in C(T)$ we define the set of its maximizers

$$
\text { set- } \operatorname{argmax}(f):=\left\{t \in T: f(t)=\max _{t \in T} f(t)\right\},
$$

which is a non-empty compact subset of $T$, since $T$ is compact and $f$ is continuous. Thus, we have a well-defined map set-argmax : $C(T) \rightarrow K(T)$ into $K(T)$, the set of non-empty compact subsets of $T$. By choosing a countable dense subset $S$ of the compact metrizable space $T$, we can express set-argmax $(f)$ as

$$
\operatorname{set}-\operatorname{argmax}(f)=\bigcap_{s \in S}\{t \in T: f(t) \geq f(s)\}
$$

since $f$ is continuous. Now, for each $s \in S$, the set $A_{s}(f):=\{t \in T: f(t) \geq f(s)\}$ is a non-empty compact subset of $T$, since $T$ is compact and $f$ is continuous. Moreover the assignment $C(T) \rightarrow K(T)$ with $f \mapsto A_{s}(f)$ is measurable with respect to the Borel- $\sigma$-algebra of the Fell topology on $K(T)$. To see this, e.g. write $A_{s}(f)=$ $\left\{t \in T: f_{s}(t) \geq 0\right\}$ as upper level set of the continuous function $f_{s}(t)=f(t)-$ $f(s)$, which depends continuously on $f \in C(T)$ (cf. [65, Example 1.2]). Hence, also the countable intersection set-argmax $(f)=\bigcap_{s \in S} A_{s}(f): C(T) \rightarrow K(T)$ is measurable with respect to the Borel- $\sigma$-algebra of the Fell topology on $K(T)$ (cf. 
[65. Theorem 2.25]). Because $T$ is compact, the Fell topology on $K(T)$ coincides with the topology of the Hausdorff distance $\rho_{d}$ with respect to the metric $d$ on $T$ (cf. [3, Theorem 3.93]). Thus, set-argmax $: C(T) \rightarrow K(T)$ is measurable with respect to the Borel- $\sigma$-algebra of the Hausdorff distance $\rho_{d}$. Further, the map

$$
i: T \rightarrow K(T), \quad i(t)=\{t\}
$$

embeds $(T, d)$ isometrically into $K(T)$ as a closed (hence measurable) subset of $K(T)$ (cf. [3, Lemma 3.78]). We may conclude that

$$
C_{\text {amax }}(T)=\text { set-argmax }^{-1}(i(T))
$$

is measurable (with respect to the Borel- $\sigma$-algebra of the supremum norm). Moreover, the map

$$
\operatorname{argmax}:=i^{-1} \text { o set-argmax }: C_{\operatorname{amax}}(T) \rightarrow T
$$

is well-defined. Let $A \subset T$ be closed (and hence compact). Then $i(A) \subset K(T)$ is also compact, since $i$ is continuous. Therefore,

$$
\operatorname{argmax}^{-1}(A)=\text { set-argmax }^{-1}(i(A)) \subset C_{\operatorname{amax}}(T)
$$

is measurable, which shows that the map argmax is measurable.

Proof of Theorem 5.3.1. We take the proof of Theorem 14 in [52] as a guideline adding some subtle changes. Let $\left(\Omega, \mathcal{A}, \mathbb{P}_{W}\right)$ denote the probability space corresponding to the Gaussian process $W: \Omega \rightarrow C(T)$. The process $\xi: \Omega \rightarrow C(T)$ with $\xi(t):=W(t)-\sigma^{2}(t) / 2$ is also Gaussian and continuous. A short computation shows that

$$
\operatorname{Var}(\xi(s)-\xi(t))=\gamma(s, t)
$$

Because of 5.4, we have that $\operatorname{Stab}\left(t_{o}\right) \subset \operatorname{Stab}\left(g t_{o}\right)=g \operatorname{Stab}\left(t_{o}\right) g^{-1}$ for all $g \in G$. Hence, $\operatorname{Stab}\left(t_{o}\right) \subset G$ is a normal subgroup of $G$. By Lemma 5.3.4, we have that the map $G / \operatorname{Stab}\left(t_{o}\right) \rightarrow G t_{o}$ given by $[g] \mapsto g t_{o}$ is well-defined and a homeomorphism. In particular

$$
H_{o}:=G / \operatorname{Stab}\left(t_{o}\right) \cong G t_{o}
$$

is a compact metric group and $G$ acts continuously on $H_{o}$ by left multiplication: 
$g[h]:=[g h]$. Moreover, the following map is well-defined and continuous

$$
()_{o}: C(T) \rightarrow C\left(H_{o}\right), \quad f \mapsto f_{o}, \quad \text { where } f_{o}([g]):=f\left(g t_{o}\right)
$$

Applying this map ()o to $\xi$ yields a sample continuous Gaussian process $\xi_{o}: \Omega \rightarrow$ $C\left(H_{o}\right)$. Since $H_{o}$ is compact metric, each sample path of $\xi_{o}$ attains its maximum and the set $C_{\operatorname{amax}}\left(H_{o}\right)$ of continuous functions on $H_{o}$ with unique maximizer is measurable, cf. Lemma 5.3.5. Further, due to (5.7) and (5.6) we have

$$
\operatorname{Var}\left(\xi_{o}\left(\left[g_{1}\right]\right)-\xi_{o}\left(\left[g_{2}\right]\right)\right)=\gamma\left(g_{1} t_{o}, g_{2} t_{o}\right) \neq 0 \quad \forall\left[g_{1}\right] \neq\left[g_{2}\right]
$$

Thus, [53, Lemma 2.6.] ensures that $\mathbb{P}_{W}\left(\xi_{o}^{-1}\left(C_{\text {amax }}\left(H_{o}\right)\right)\right)=1$. So, the Gaussian process $W$ allows for an undistinguishable process $W^{*}$ on $\Omega$ by replacing the sample paths on the complement of $\xi_{o}^{-1}\left(C_{\mathrm{amax}}\left(H_{o}\right)\right)$ in $\Omega$ by some continuous path, such that the process $\xi_{o}^{*}([g]):=\xi^{*}\left(g t_{o}\right):=W^{*}\left(g t_{o}\right)-\sigma^{2}\left(g t_{o}\right) / 2$ attains its maximum at only one point. We denote the preimage of $C_{\operatorname{amax}}\left(H_{o}\right)$ under the continuous map (5.8) by $C_{\text {amax }, o}(T)$. Then, $\xi^{*}$ takes its values only in $C_{\text {amax }, o}(T) \subset C(T)$.

In what follows, we will show that the corresponding Brown-Resnick process $\eta^{*}$ defined through Theorem 5.1.2 (which has the same law as $\eta$ ) has a Mixed Moving Maxima representation. For notational convenience we will omit the index ()* henceforth. From now on, we can stick closer to the proof of Theorem 14 in [52].

Firstly, Lemma 5.3.5 ensures that the argmax-map argmax : $C_{\text {amax }}\left(H_{o}\right) \rightarrow H_{o}$ is measurable. This is the crucial point when verifying that the following maps are measurable:

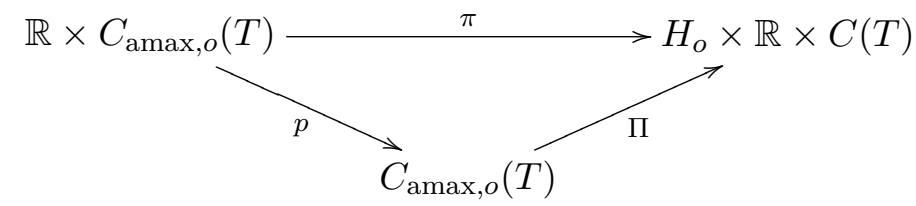

$$
\begin{aligned}
\pi(u, \xi):=\left(\left[g^{\prime}\right], y^{\prime}, f^{\prime}\right), \quad \text { where } \quad\left[g^{\prime}\right] & :=\operatorname{argmax}\left(\xi_{o}\right), \\
y^{\prime} & :=u+\max \left(\xi_{o}\right), \\
f^{\prime}(t) & :=u+\xi\left(g^{\prime} t\right)-y^{\prime} \\
{\left[g^{\prime \prime}\right] } & :=\operatorname{argmax}\left(f_{o}\right), \\
y^{\prime \prime} & :=\max \left(f_{o}\right), \\
f^{\prime \prime}(t) & :=f\left(g^{\prime \prime} t\right)-y^{\prime \prime}
\end{aligned}
$$

$p(u, \xi):=u+\xi$ 
Note that $p$ is well-defined and that the continuous functions $f^{\prime} \in C(T)$ and $f^{\prime \prime} \in C(T)$ are only well-defined $\operatorname{since} \operatorname{Stab}\left(t_{o}\right) \subset \operatorname{Stab}(t)$ for all $t \in T$ (as assumed, see (5.4)). The above diagram commutes, i.e. $\pi=\Pi \circ p$ and

$$
\Pi\left(g^{*} f\right)=\left(g^{-1}\left[g^{\prime \prime}\right], y^{\prime \prime}, f^{\prime \prime}\right) \quad \forall g \in G,
$$

where $g^{*} f$ denotes the function on $T$ given by $g^{*} f(t)=f(g t)$, which belongs again to $C_{\mathrm{amax}, o}(T)$.

Now, the process $\eta$ is constructed by (5.1) from a Poisson point process $\left(U_{n}, \xi_{n}\right)_{n=1}^{\infty}$ on $\mathbb{R} \times C(T)$ with intensity measure $e^{-u} \mathrm{~d} u \times d \mathbb{P}_{\xi}$ where $\mathbb{P}_{\xi}$ is the law of $\xi$ on $C_{\text {amax }, o}(T)$. This process transforms under $\pi$ to a Poisson point process on $H_{o} \times$ $T \times C(T)$ with intensity measure

$$
\Psi(A)=\int_{\pi^{-1}(A)} e^{-u} \mathrm{~d} u \times \mathrm{d} \mathbb{P}_{\xi}=\int_{\mathbb{R}} e^{-u} \mathbb{P}\left[\left(\left[g^{\prime}\right], y^{\prime}, f^{\prime}\right) \in A\right] \mathrm{d} u
$$

for sets $A$ in the Borel- $\sigma$-algebra of $H_{o} \times T \times C(T)$, cf. the mapping theorem for Poisson point processes [54]. By the same computation as in [52] it follows that

$$
\Psi(\{([h], y+z, f):([h], y, f) \in A\})=e^{-z} \Psi(A) \quad \forall z \in \mathbb{R} .
$$

Because $\gamma$ is $G$-invariant (see $(5.2)$ ) and $\pi$ factorizes by the above diagram and $\Pi$ commutes with the $G$ action in the sense of (5.9), Theorem 1 in [49] implies that

$$
\Psi\left(\left\{\left(g^{-1}[h], y, f\right):([h], y, f) \in A\right\}\right)=\Psi(A) \quad \forall g \in G
$$

Further, we have that

$$
\begin{aligned}
\Psi\left(H_{o} \times[0,1] \times C(T)\right) & =\int_{\mathbb{R}} e^{-u} \mathbb{P}\left(y^{\prime} \in[0,1]\right) \mathrm{d} u=\int_{\mathbb{R}} e^{-u} \mathbb{P}\left(u+\max \left(\xi_{o}\right) \in[0,1]\right) \mathrm{d} u \\
& \leq \int_{\mathbb{R}} e^{v} \mathbb{P}\left(\max \left(\xi_{o}\right) \geq v\right) \mathrm{d} v \leq \int_{\mathbb{R}} e^{v} \mathbb{P}\left(\left\|\xi_{o}\right\|_{C\left(H_{o}\right)} \geq v\right) \mathrm{d} v<\infty
\end{aligned}
$$

where the last inequality follows from the asymptotics of Corollary 3.2 in [56].

Following [52] we introduce the measure $\Psi_{A}$ on $H_{O} \times \mathbb{R}$ for a measurable subset $A \subset C(T)$ via $\Psi_{A}(B)=\int_{B \times A} e^{y} \mathrm{~d} \Psi([g], y, f)$. Because of (5.11) the measure $\Psi_{A}$ is $G$-invariant (or equivalently $H_{o}$-invariant) in the first component $H_{o}$ and due to 5 (5.10) it is translation invariant in the second component $\mathbb{R}$. Hence $\Psi_{A}$ is a multiple of the product measure which is built from the (probability) Haar measure $\tilde{\mu}$ on $H_{o}$ (see Lemma 5.3.4 and the Lebesgue measure on $\mathbb{R}$. Therefore, we write $\mathrm{d} \Psi_{A}=$ $\mathbb{Q}(A) \tilde{\mu}(\mathrm{d}[g]) \mathrm{d} y$ for some positive constant $\mathbb{Q}(A)$, where the assignment $A \mapsto \mathbb{Q}(A)$ 
also defines a finite measure on $C(T)$. Setting $c:=\mathbb{Q}(C(T))$ and normalizing $\mathbb{Q}$ to a probability measure $\mathbb{Q}^{\prime}:=\mathbb{Q} / c$, we arrive at $\mathrm{d} \Psi=c e^{-y} \tilde{\mu}(\mathrm{d}[g]) \mathrm{d} y \mathrm{~d} \mathbb{Q}^{\prime}$.

By construction, we get that

$$
\eta(t)=\bigvee_{n=1}^{\infty} U_{n}+\xi_{n}(t)=\bigvee_{n=1}^{\infty} y_{n}^{\prime \prime \prime}+f_{n}^{\prime \prime \prime}\left(\left[g^{\prime \prime \prime}\right]_{n}^{-1} t\right)
$$

where the triple

$$
\left(\left[g^{\prime \prime \prime}\right]_{n}, y_{n}^{\prime \prime \prime}, f_{n}^{\prime \prime \prime}\right)=\left(\left[g^{\prime}\right]_{n}, y_{n}^{\prime}-\log (c), f_{n}^{\prime}+\log (c)\right)
$$

originates from the Poisson point process $\left(\left[g^{\prime}\right]_{n}, y_{n}^{\prime}, f_{n}^{\prime}\right)_{n=1}^{\infty}$ with intensity measure $\mathrm{d} \Psi=c e^{-y} \tilde{\mu}(\mathrm{d}[g]) \mathrm{d} y \mathrm{~d} \mathbb{Q}^{\prime}$. As in [52] we may conclude that, $\left(\left[g^{\prime \prime \prime}\right]_{n}, y_{n}^{\prime \prime \prime}, f_{n}^{\prime \prime \prime}\right)_{n=1}^{\infty}$ is a Poisson point process on $H_{o} \times \mathbb{R} \times C(T)$ with intensity measure $e^{-y} \tilde{\mu}(\mathrm{d}[g]) \mathrm{d} y \mathrm{~d} \mathbb{Q}^{*}$, where $\mathbb{Q}^{*}$ is the law of $f^{\prime \prime \prime}=f^{\prime}+\log c$. Thus, the representation 5.12 arises from the Poisson point process $\left(\left[g^{\prime \prime \prime}\right]_{n}, y_{n}^{\prime \prime \prime}\right)_{n=1}^{\infty}$ on $H_{o} \times \mathbb{R}$ with intensity $e^{-y} \tilde{\mu}(\mathrm{d}[g]) \mathrm{d} y$ and independently sampled i.i.d. copies $f_{n}^{\prime \prime \prime}$ from the probability measure $\mathbb{Q}^{*}$ on $C(T)$. Due to the Gumbel marginals of $\eta$,

$$
\int_{C(T)} \int_{H_{o}} e^{f\left([g]^{-1} t\right)} \tilde{\mu}(\mathrm{d}[g]) \mathbb{Q}^{*}(\mathrm{~d} f)=-\log \mathbb{P}(\eta(t) \leq 0)=1 \quad \forall t \in T,
$$

i.e. the representation (5.12) satisfies (5.3). Hence, (5.12) is a Mixed Moving Maxima representation of $\eta$ with respect to the group action of $H_{o}$. Furthermore, the measure $\tilde{\mu}$ on $H_{o}$ is the pushforward measure of the (probability) Haar measure $\mu$ on $G$ (see Lemma 5.3.4). This implies that the representation

$$
\eta(t)=\bigvee_{n=1}^{\infty} y_{n}^{\prime \prime \prime}+f_{n}^{\prime \prime \prime}\left(g_{n}^{\prime \prime \prime}-1\right)
$$

where $\left(g_{n}^{\prime \prime \prime}, y_{n}^{\prime \prime \prime}\right)_{n=1}^{\infty}$ is a Poisson point process on $G \times \mathbb{R}$ with intensity $e^{-y} \mu(\mathrm{d} g) \mathrm{d} y$ and $f_{n}^{\prime \prime \prime}$ as above, is also a Mixed Moving Maxima representation of $\eta$, now with respect to the group action of $G$.

\subsection{Extremal log-Gaussian representation on the sphere}

We consider the Brown-Resnick process $\eta$ from Theorem 5.1.2 on the 2-sphere $T=$ $S^{2}$ w.r.t. the action of the (orientation preserving) isometry group $G=\operatorname{SO}(3)$. Then the involved variogram $\gamma(s, t)$ necessarily depends only on the angular distance $\alpha(s, t)$ between points $s$ and $t$ on the sphere, since $\gamma$ is symmetric and for any fixed 
distance $d$ the group $\mathrm{SO}(3)$ acts transitively on $\left\{\{s, t\}: s, t \in S^{2}\right.$ with $\left.\alpha(s, t)=d\right\}$. It is known that such variograms can be represented as $\gamma(s, t)=c_{o}-C(s, t)$ for some covariance function on the sphere [43, Remark 2], where $c_{o} \geq \int_{o}^{\pi} \gamma(\alpha) \sin (\alpha) \mathrm{d} \alpha$. Thus, the Gaussian process $W$ in Theorem 5.1 .2 with variogram $\gamma$ can always be chosen to have covariance $C(s, t) / 2$, which depends on the angular distance only. Likewise, the resulting process $\xi(t)=W(t)-c_{o} / 4$ has the same covariance function as the process $W$ with a different mean and the process $\eta$ can be built from $\xi$ via 5.1. Such constructions are considered in the case of $T=\mathbb{R}^{d}$, for instance, in [83. Theorem 2] for Fréchet marginals. Analogously to the name extremal Gaussian process and extremal binary Gaussian process as in Example 1.2.2, it is concise to call this representation of the process $\eta$ extremal log-Gaussian process, since the stationary Gaussian process in the construction of extremal Gaussian processes is simply replaced by an $\mathrm{SO}(3)$-invariant log-Gaussian process.

In particular, the representation (5.1) with $\mathrm{SO}(3)$-invariant $\xi$ (instead of an "intrinsically $\mathrm{SO}(3)$-invariant" $\xi$ ) is an important observation if one is interested in simulating the Brown-Resnick process $\eta$ on the sphere, as the "intrinsic" version can always be avoided.

Finally, note that in general, bounded variograms $\gamma$ do not need to be representable as $\gamma(s, t)=c_{o}-C(s, t)$ for some constant $c_{o}$ and some covariance function $C$, as the example in [5, 3.2.5 Remark] indicates, whereas such a representation can still be obtained, when group invariant variograms on compact homogeneous spaces are considered (cf. [101, p. 619]). 


\section{Concluding remarks}

My original interest of research was in a complete characterization of the class of tail correlation functions. At the early stages of this project I realized that the link to the extremal coefficient functions was a promising starting point. Chapter 2 deals now with this approach and does not only prepare the results in Chapter 3 , but contains many observations that are of independent interest. For instance, the construction of a process parameterized by its ECF in Theorem 2.3.5 and the sharp inequality of Corollary 2.6.8 have been established. The characterization of the set of TCFs as an infinite-dimensional polytope and the observations on partition TCFs in Chapter 3 have become the closest answer to the original characterization problem for TCFs. Note that these results are not restricted to the class of max-stable processes, since Chapter 3 also takes into account all TCFs of not necessarily max-stable processes as well as the closure of normalized covariance functions of binary processes.

Moreover, the Gneiting class $H_{\infty}$ is, in my opinion, a satisfactory analogue in the context of TCFs to the class $\Phi_{\infty}$ in the context of correlation functions, where $\Phi_{\infty}$ denotes the radial continuous correlation functions that are admissable on $\mathbb{R}^{d}$ for all dimensions $d$. Likewise, the class $H_{\infty}$ contains radial continuous TCFs that are admissable on $\mathbb{R}^{d}$ for all dimensions $d$. It is known that functions from $\Phi_{\infty}$ take the form $\varphi\left(r^{2}\right)$, where $\varphi$ is a completely monotone function on $[0, \infty)$ with $\varphi(0)=1$. The functions $\varphi$ from $H_{\infty}$ are continuous with $\varphi(0)=1$ and decreasing to 0 , such that $-\varphi^{\prime}(\sqrt{\cdot})$ is completely monotone on $(0, \infty)$. Convex combinations of functions from $H_{\infty}$ and the constant function 1 form the richest class of TCFs we know that does not depend on the specific dimension of the surrounding space (see Chapter 4 ). Therefore, I think that the class of variance-mixed Brown-Resnick processes which realizes the class $H_{\infty}$ offers a useful flexibility worth studying.

Further, advanced TCFs with desired properties (such as compact support) can be constructed from the basic construction principles for TCFs from Chapters 3 
and 4. The variance-mixing in the construction of Brown-Resnick processes can also be combined with the generalizations of Brown-Resnick processes from Chapter 5 . For instance, there exists a variance-mixed Brown-Resnick process on the 2-sphere $S^{2}$ with TCF $\chi(s, t)=1-2 \pi^{-1} \arctan \left(\alpha(s, t)^{q} / a\right)$ for $q \in(0,0.5]$ and $a>0$, which depends solely on the angular distance $\alpha(s, t)$ between points $s$ and $t$.

Finally, we collect some questions that remained unsolved. Three open problems have already been formulated in Remarks 2.6.10, 3.4.8 and 3.6.6. Further open questions concerning the characterization of TCFs are as follows.

A complete list of all higher dimensional inequalities describing the class of TCFs seems hard to achieve. However, the observation that partition TCFs are precisely the vertices of $\operatorname{TCF}(M)$ for $|M| \leq 4$ in Chapter 3 is at first sight promising for a characterization of the general vertex set. It is unclear if this is a low-dimensional phenomenon or a deeper structural result.

In Chapter 3 it was shown that two TCFs can be multiplied and the result is again a TCF. However, it is not clear which products of a TCF and an arbitrary non-negative correlation function are again a TCF. For instance, it would be nice to incorporate a damped oscillation. Multiplication with a periodic zigzag function (which is an admissable TCF on $\mathbb{R}$ ) is rather unsatisfactory compared to the multiplication with a cosine that is normalized to a non-negative correlation function (which is not a TCF).

In Remark 4.4.11 it is noted that the function $\chi(t):=1-\beta \cdot t$ that decreases linearly in the radius $t$ is an admissible TCF on the $d$-dimensional ball $B_{r}^{d}(o)$ of radius $r$ if $\beta \in\left[0, \beta_{d} / r\right]$ with $\beta_{d}$ as in (4.19). With regard to results in [38], where it is shown that $\varphi(t)=1-\alpha \cdot t$ is positive definite on the $d$-dimensional ball of radius $r$ if and only if $\alpha \in\left[0,2 \beta_{d} / r\right]$, it seems likely that the bound in Remark 4.4.11 is sharp. 


\section{A. Appendix}

\section{A.1. Harmonic analysis on abelian semigroups}

We revise several notions and results from harmonic analysis on abelian semigroups referring mostly to [5] and [65] for the notation in Equation (A.1). For our purposes it suffices to consider only real-valued functions. This section is particularly relevant for Chapter 2 .

Let $(S, \circ, e)$ be an abelian semigroup (meaning that $S$ is a non-empty set with an associative and commutative composition $\circ$ on $S$ and a neutral element $e$ ). The composition of several elements $s_{i}$ for $i \in I$ will be abbreviated by $\bigcirc_{i \in I} s_{i}$.

Definition A.1.1 (positive definite and negative definite functions in the semigroup sense). A function $\varphi: S \rightarrow \mathbb{R}$ is called positive definite (in the semigroup sense) if for all $n \geq 1,\left\{s_{1}, \ldots, s_{n}\right\} \subset S,\left\{a_{1}, \ldots, a_{n}\right\} \subset \mathbb{R}$

$$
\sum_{j, k=1}^{n} a_{j} a_{k} \varphi\left(s_{j} \circ s_{k}\right) \geq 0
$$

A function $\psi: S \rightarrow \mathbb{R}$ is called negative definite (in the semigroup sense) if for all $n \geq 2,\left\{s_{1}, \ldots, s_{n}\right\} \subset S,\left\{a_{1}, \ldots, a_{n}\right\} \subset \mathbb{R}$ with $\sum_{j=1}^{n} a_{j}=0$

$$
\sum_{j, k=1}^{n} a_{j} a_{k} \psi\left(s_{j} \circ s_{k}\right) \leq 0 .
$$

Remark A.1.2. More generally, we could define positive and negative definite functions for semigroups $S$ with involution $*: S \rightarrow S$ (i.e. $*^{2}=\mathrm{id}_{S}$ ) by replacing the term $\varphi\left(s_{j} \circ s_{k}\right)$ by $\varphi\left(s_{j} \circ *\left(s_{k}\right)\right)$ in the definition above. Instead, we work here only with the identity as involution. If $S$ was an abelian group (where each element $s$ 
has an inverse $-s)$, the choice $*(s)=-s$ would be in accordance with a definition of positive definite functions on abelian groups that might be more familiar to the reader.

We write $P(S)$ and $N(S)$ for the set of positive definite and negative definite functions on $S$, respectively. Further, $P^{b}(S)$ and $N^{l}(S)$ shall denote the set of bounded positive definite functions and lower bounded negative definite functions. Clearly, all these properties (positive and negative definiteness, being bounded or having a lower bound) respect convex combinations and scaling. So $P(S), P^{b}(S)$, $N(S), N^{l}(S)$ are convex cones.

The cones $P(S)$ and $P^{b}(S)$ are also closed under pointwise multiplication. If $\varphi_{1}$ and $\varphi_{2}$ are positive definite functions (and additionally bounded), so is $\varphi_{1} \cdot \varphi_{2}$. The cones $N(S)$ and $N^{l}(S)$ are closed under adding a constant. If $\psi_{1}$ is negative definite (and additionally lower bounded), so is $\psi+c$ for any constant $c \in \mathbb{R}$. The following lemma gives a relation between $P(S)$ and $N(S)$.

Lemma A.1.3 ([5], 3.2.1. and 3.2.2.). For a function $\psi: S \rightarrow \mathbb{R}$ the following are equivalent:

(i) $\psi \in N(S)$

(ii) $\exp (-t \psi) \in P(S)$ for all $t>0$.

(iii) The function $(s, t) \rightarrow \psi(s)+\psi(t)-\psi(s \circ t)-\psi(e)$ is positive definite as a kernel on $S \times S$.

The functions from the convex cones $P^{b}(S)$ and $N^{l}(S)$ allow for an integral representation in terms of bounded semicharacters.

Definition A.1.4. A bounded semicharacter $\rho: S \rightarrow[-1,1]$ is a function that satisfies $\rho(e)=1$ and $\rho(s \circ t)=\rho(s) \rho(t)$ for $s, t \in S$.

The set of all bounded semicharacters is denoted $\widehat{S}$. In particular each bounded semicharacter is a positive definite function. The set $\widehat{S}$ forms itself a semigroup under pointwise multiplication and neutral element the semicharacter $\rho \equiv 1$. We endow $\widehat{S} \subset[-1,1]^{S}$ with the product topology which makes $\widehat{S}$ a topological semigroup. As a topological space $\widehat{S}$ is a completely regular compact Hausdorff space. The following representation theorem holds.

Theorem A.1.5 ([5], 4.2.8. and 4.3.2. Integral representation for functions from $P^{b}(S)$ and $\left.N^{l}(S)\right)$. If $\varphi \in P^{b}(S)$, the function $\varphi$ uniquely determines a positive Radon measure $\mu$ on $\widehat{S}$, such that

$$
\varphi(s)=\int_{\widehat{S}} \rho(s) \mu(\mathrm{d} \rho) .
$$


If $\psi \in N^{l}(S)$, the function $\psi$ uniquely determines an additive function $q: S \rightarrow[0, \infty)$ and a positive Radon measure $\mu$ on $\widehat{S} \backslash\{1\}$ (called Lévy measure) such that

$$
\psi(s)=\psi(0)+q(s)+\int_{\widehat{S} \backslash\{1\}}(1-\rho(s)) \mu(\mathrm{d} \rho) .
$$

Here $q(s)=\lim _{n \rightarrow \infty} \frac{\psi(n s)}{n}$ for $s \in S$. If $q$ is identically zero, then $\psi$ is bounded if and only if $\mu(\widehat{S} \backslash\{1\})<\infty$.

Moreover, if $\varphi: S \rightarrow \mathbb{R}$ is positive definite (and bounded) and $c \in \mathbb{R}$ a constant, then trivially, the function $\psi=c-\varphi$ is negative definite (and bounded). But also conversely, any bounded negative definite function $\psi: S \rightarrow \mathbb{R}$ has the form $\psi=c-\varphi$ for some positive definite $\varphi$ and a constant $c$ (cf. [5, 4.3.15.]).

For a function $f: S \rightarrow \mathbb{R}$ and elements $s_{1}, s \in S$ we use the notation (cf. 65, p. 7])

$$
\left(\Delta_{s_{1}} f\right)(s):=f(s)-f\left(s \circ s_{1}\right) .
$$

Note that the operators $\Delta_{s_{1}}$ and $\Delta_{s_{2}}$ commute with each other.

Definition A.1.6 (completely monotone and completely alternating functions). A function $\varphi: S \rightarrow \mathbb{R}$ is called completely monotone if for all $n \geq 0,\left\{s_{1}, \ldots, s_{n}\right\} \subset S$ and $s \in S$

$$
\left(\Delta_{s_{1}} \Delta_{s_{2}} \ldots \Delta_{s_{n}} \varphi\right)(s)=\sum_{I \subset\{1, \ldots, n\}}(-1)^{|I|} \varphi\left(s \circ \bigcirc_{i \in I} s_{i}\right) \geq 0
$$

A function $\psi: S \rightarrow \mathbb{R}$ is called completely alternating if for all $n \geq 1,\left\{s_{1}, \ldots, s_{n}\right\} \subset$ $S$ and $s \in S$

$$
\left(\Delta_{s_{1}} \Delta_{s_{2}} \ldots \Delta_{s_{n}} \psi\right)(s)=\sum_{I \subset\{1, \ldots, n\}}(-1)^{|I|} \psi\left(s \circ \bigcirc_{i \in I} s_{i}\right) \leq 0
$$

Lemma A.1.7 ([5], 4.6.6.). Let $G$ be a generator for the semigroup $S$. Then a function $\psi: S \rightarrow \mathbb{R}$ is completely alternating if and only if for all $n \geq 1,\left\{s_{1}, \ldots, s_{n}\right\} \subset G$ and $s \in S$

$$
\left(\Delta_{s_{1}} \Delta_{s_{2}} \ldots \Delta_{s_{n}} \psi\right)(s)=\sum_{I \subset\{1, \ldots, n\}}(-1)^{|I|} \psi\left(s \circ \bigcirc_{i \in I} s_{i}\right) \leq 0
$$

We write $M(S)$ and $A(S)$ for the set of completely monotone and completely alternating functions on $S$, respectively. They can be characterized as follows. Let $\widehat{S}_{+}$denote the set of bounded semicharacters which attain values only in $[0,1]$. 
Theorem A.1.8 ([5], 4.6.5. and 4.6.7.). The set $M(S)$ is an extreme convex subcone of $P^{b}(S)$. A function $\varphi \in P^{b}(S)$ is completely monotone if and only if the corresponding measure $\mu$ from Theorem A.1.5 is concentrated on $\widehat{S}_{+}$.

The set $A(S)$ is an extreme convex sub-cone of $N^{l}(S)$. A function $\psi \in N^{l}(S)$ is completely alternating if and only if the corresponding Lévy measure $\mu$ from Theorem A.1.5 is concentrated on $\widehat{S}_{+} \backslash\{1\}$.

In particular, if $S$ is 2-divisible (i.e. every element $s \in S$ can be decomposed into $s=s^{\prime}+s^{\prime}$ for some $s^{\prime} \in S$ ), positive definite functions are automatically positive (cf. [5, 4.1.6.]) and thus, $\widehat{S}=\widehat{S}_{+}$. Consequently, $M(S)=P^{b}(S)$ and $A(S)=N^{l}(S)$ if $S$ is 2-divisible.

The cone $M(S)$ is closed under pointwise multiplication. If $\varphi_{1}$ and $\varphi_{2}$ are completely monotone functions, so is $\varphi_{1} \cdot \varphi_{2}$. The cone $A(S)$ is closed under adding a constant. If $\psi_{1}$ is completely alternating, so is $\psi+c$ for any constant $c \in \mathbb{R}$.

Lemma A.1.9 ([5], 4.6.10.). For a function $\psi: S \rightarrow \mathbb{R}$ the following are equivalent:

(i) $\psi \in A(S)$

(ii) $\exp (-t \psi) \in M(S)$ for all $t>0$.

Idempotent semigroups (cf. [5, 4.4.16.]) Let $S$ be an idempotent semigroup, i.e. every element $s \in S$ satisfies $s \circ s=s$. Then $S$ is also 2-divisible and $M(S)=P^{b}(S)$ and $A(S)=N^{l}(S)$. Further, we can define a partial order on $S$ by saying that $s \leq t$ if $s \circ t=t$. With respect to this partial order positive definite functions $\varphi$ are automatically decreasing satisfying $0 \leq \varphi(s) \leq \varphi(e)$, whereas negative definite functions $\psi$ are always increasing, thus bounded from below by $\psi(e) \leq \psi(s)$. It follows that

$$
M(S)=P^{b}(S)=P(S) \quad \text { and } \quad A(S)=N^{l}(S)=N(S)
$$

Consider a semicharacter $\rho \in \widehat{S}$. We see directly from the definition that it takes values in $\{0,1\}$ if $S$ is idempotent. Set $I:=\rho^{-1}(\{1\}) \subset S$. Then $I$ is a sub-semigroup of $S$ which is hereditary on the left, that is: whenever $s \leq t$ and $t \in I$ it follows that $s \in I$. In fact, if we denote $\mathscr{S}$ the set of sub-semigroups of $S$ which are hereditary on the left, we have an isomorphism of semigroups $(\widehat{S}, \cdot 1) \cong(\mathscr{S}, \cap, S)$ where the map and its inverse are given by $\rho \mapsto \rho^{-1}(\{1\})$ and $I \mapsto \mathbb{1}_{I}$. This is indeed an isomorphism of topological semigroups, if $\mathscr{S}$ is endowed with the topology generated by the maps $\left(\delta_{s}\right)_{s \in S}$ where $\delta_{s}(I)=\mathbb{1}_{I}(s)$. Moreover, if $\psi \in N(S)=N^{l}(S)$, the corresponding $q$ from Theorem A.1.5 is identically zero, since $q(s)=\lim _{n \rightarrow \infty} \frac{\psi(n s)}{n}=\lim _{n \rightarrow \infty} \frac{\psi(s)}{n}=$ 0. Theorem A.1.5 simplifies to the following version. 
Theorem A.1.10 ([5], 4.4.17.). Let $(S, \circ, e)$ be an idempotent semigroup.

If $\varphi \in P(S)=M(S)$, the function $\varphi$ uniquely determines a positive Radon measure $\mu$ on $\widehat{S}=\mathscr{S}$, such that

$$
\varphi(s)=\mu(\{\rho \in \widehat{S}: \rho(s)=1\})=\mu(\{I \in \mathscr{S}: s \in I\}) .
$$

If $\psi \in N(S)=A(S)$, the function $\psi$ uniquely determines a positive Radon measure $\mu$ on $\widehat{S} \backslash\{1\}=\mathscr{S} \backslash\{S\}$ such that

$$
\psi(s)=\psi(0)+\mu(\{\rho \in \widehat{S} \backslash\{1\}: \rho(s)=0\})=\psi(0)+\mu(\{I \in \mathscr{S} \backslash\{S\}: s \notin I\}) .
$$

The function $\psi$ is bounded if and only if $\mu(\widehat{S} \backslash\{1\})=\mu(\mathscr{S} \backslash\{S\})<\infty$.

\section{A.2. Monotonicity properties of continuous functions}

This section is particularly relevant for Chapter 4 We consider continuous functions on intervals of the real line and define certain monotonicity properties. The definitions made here are in accordance with the previous Section A.1 if we consider functions on the interval $[0, \infty)$ when viewed as a semigroup $([0, \infty),+, 0)$. Therefore, we refer to [5, 4.6.13.].

Definition A.2.1 (cf. [62, 100]). Let $(a, b)$ be an open interval of the real line with $a, b \in[-\infty, \infty]$ and $n \in \mathbb{N}$. A real-valued function $f$ is called $n$-times monotone on $(a, b)$, where $n \geq 2$, if it is differentiable up to order $n-2$ and $(-1)^{k} f^{(k)}$ is non-negative, non-increasing and convex on $(a, b)$ for $k=0,1, \ldots, n-2$. If $n=1$, we simply require $f$ to be non-negative and non-increasing on $(a, b)$.

In case $n \geq 2$, we could have equivalently demanded that $(-1)^{k} f^{(k)}(x) \geq 0$ for $x \in(a, b)$ and $k=0,1, \ldots, n-2$ and that $(-1)^{(n-2)} f^{(n-2)}(x)$ is non-negative and convex in $(a, b)$. Thus, it becomes more apparent that for $n \rightarrow \infty$ we arrive at the definition of completely monotone functions.

Definition A.2.2 ([99], Chapter IV). ) Let $(a, b)$ be an open interval of the real line with $a, b \in[-\infty, \infty]$. A real-valued function $f$ is called completely monotone on $(a, b)$ (resp. absolutely monotone on $(a, b)$ if it has derivatives of all orders and if

$$
(-1)^{k} f^{(k)}(x) \geq 0 \quad\left(\text { resp. } f^{(k)}(x) \geq 0\right)
$$

for all $x \in(a, b)$ and $k \in \mathbb{N} \cup\{0\}$.

Finally, we extend these notions also to closed or half-open intervals. 
Definition A.2.3. Let $I$ be an interval of the real line. We call a real-valued function $f$ n-times monotone on I (resp. completely monotone on I / absolutely monotone on $I$ ) if $f$ has this property when restricted to the interior $\stackrel{\circ}{I}$ and if $f$ is continuous at the boundary points of $I$.

Clearly, $f(x)$ is absolutely monotone on $I$ if and only if $f(-x)$ is completely monotone on the reflected interval $-I$. In the literature, the focus often lies on the intervals $I=(0, \infty)$ or $I=[0, \infty)$, since completely monotone functions on $[0, \infty)$ are precisely the functions $f$, such that $f\left(\|\cdot\|^{2}\right)$ is positive definite on $\mathbb{R}^{d}$ for all dimensions $d$ (cf. e.g. [5, 5.1.5 and 5.1.6.]). Such functions are characterized as Laplace transforms of non-decreasing functions (or positive measures), cf. [99, Chapter IV Theorem 12].

Theorem A.2.4 (Bernstein). A function $f:(0, \infty) \rightarrow \mathbb{R}$ is completely monotone on $(0, \infty)$ if and only if it has an integral representation of the form

$$
f(x)=\int_{[0, \infty)} \exp (-t x) \mathrm{d} F(t)
$$

for some non-decreasing function $F:[0, \infty) \rightarrow \mathbb{R}$, such that the integral converges for $x \in(0, \infty)$. Furthermore, the function $f$ can be extended continuously to $[0, \infty)$ - and thus, is completely monotone on $[0, \infty)$ - if and only if $F$ is bounded. In this case $f(0)=F(\infty)-F(0)$.

An analogous integral representation with Bernstein's theorem as the limiting case holds for $n$-times monotone functions, cf. [100. It presents $n$-times monotone functions as scale mixtures of Askey's function (cf. [37]).

Theorem A.2.5 (Williamson). A function $f:(0, \infty) \rightarrow \mathbb{R}$ is $n$-times monotone on $(0, \infty)$ if and only if it has an integral representation of the form

$$
f(x)=\int_{[0, \infty)}(1-t x)_{+}^{n-1} \mathrm{~d} F(t)
$$

for some non-decreasing function $F:[0, \infty) \rightarrow \mathbb{R}$ bounded from below. (Here $y_{+}$ denotes $\max (0, y)$.) This representation is unique in the sense that when $F$ is normalized to $F(0)=0$, the value $F(t)$ is determined at continuity points $t>0$ of $F$.

Finally, this motivates the definition of $\alpha$-times monotone functions for real $\alpha \geq 1$, which indeed extends the previous Definition A.2.1. For a geometric interpretation we refer again to [100]. 
Definition A.2.6 ([100]). Let $\alpha \geq 1$ be a real number. A real-valued function $f$ on $(0, \infty)$ is called $\alpha$-times monotone on $(0, \infty)$ if it has an integral representation of the form A.4 with $n=\alpha$ for some non-decreasing function $F:[0, \infty) \rightarrow \mathbb{R}$ with $F(0)=0$. Analogously to Definition A.2.3 we say that a real-valued function $f$ on $[0, \infty)$ is $\alpha$-times monotone on $[0, \infty)$ if $f$ has this property when restricted to $(0, \infty)$ and if $f$ is continuous at 0 .

\section{A.3. Brown-Resnick processes}

We rephrase results from [52] in our notation from Chapter 1 in order to deduce generalized versions in two respects. On the one hand we introduce an additional mixing in the variance term of Brown-Resnick processes in Example 1.2.6, on the other hand we consider other types of invariance in Chapter 5.

Proposition A.3.1 ([52], Proposition 6). Let $X=\left\{X_{t}\right\}_{t \in \mathbb{R}^{d}}$ be a simple max-stable process with spectral process $\left\{V_{t}\right\}_{t \in \mathbb{R}^{d}}=\left\{\exp \left(\xi_{t}\right)\right\}_{t \in \mathbb{R}^{d}}$. Then $X$ is stationary if and only if

$\mathbb{E}\left(\exp \left(u_{1} \xi_{t_{1}}+u_{2} \xi_{t_{2}}+\cdots+u_{n} \xi_{t_{n}}\right)\right)=\mathbb{E}\left(\exp \left(u_{1} \xi_{t_{1}+h}+u_{2} \xi_{t_{2}+h}+\cdots+u_{n} \xi_{t_{n}+h}\right)\right)$

for all $h, t_{1}, \ldots, t_{n} \in \mathbb{R}^{d}$ and $u_{1}, \ldots, u_{n} \in[0,1]$ with $\sum_{i=1}^{n} u_{i}=1$.

Theorem A.3.2 ([52], Theorem 2). Let $\left\{W_{t}\right\}_{t \in \mathbb{R}^{d}}$ be a Gaussian process with stationary increments and variance $\sigma^{2}(t)$. Set $V_{t}=\exp \left(W_{t}-\sigma^{2}(t) / 2\right)$ for $t \in \mathbb{R}^{d}$. Then $\mathbb{E} V_{t}=1$ and thus, $\left\{V_{t}\right\}_{t \in \mathbb{R}^{d}}$ is the spectral process of a simple max-stable process $X=\left\{X_{t}\right\}_{t \in \mathbb{R}^{d}}$. This process $X$ has the following properties.

a) $X$ is stationary.

b) The law of $X$ depends on the variogram $\gamma(t)=\mathbb{E}\left(W_{t}-W_{o}\right)^{2}$ only.

An additional mixing in the variance of the Gaussian process can be involved as follows.

Corollary A.3.3. Let $\left\{W_{t}\right\}_{t \in \mathbb{R}^{d}}$ be a Gaussian process with stationary increments and variance $\sigma^{2}(t)$. Independently from $W$, let $S$ be a random variable on $(0, \infty)$ with distribution function $G$ (with $G(0+)=0$ ). Set $V_{t}=\exp \left(S W_{t}-S^{2} \sigma^{2}(t) / 2\right)$ for $t \in \mathbb{R}^{d}$. Then $\mathbb{E} V_{t}=1$ and thus, $\left\{V_{t}\right\}_{t \in \mathbb{R}^{d}}$ is the spectral process of a simple max-stable process $X=\left\{X_{t}\right\}_{t \in \mathbb{R}^{d}}$. This process $X$ has the following properties.

a) $X$ is stationary. 
b) The law of $X$ depends on the variogram $\gamma(t)=\mathbb{E}\left(W_{t}-W_{o}\right)^{2}$ and the distribution function $G$ only.

Proof. For fixed $S \in(0, \infty)$ the stochastic process $\left\{S W_{t}\right\}_{t \in \mathbb{R}^{d}}$ is still Gaussian with stationary increments. Moreover, the variance of $S W_{t}$ is $\operatorname{Var}\left(S W_{t}\right)=S^{2} \sigma^{2}(t)$ and the variogram is $\mathbb{E}\left(S W_{t}-S W_{o}\right)^{2}=S^{2} \gamma(t)$. Therefore, all statements can be deduced from the analogous statement in Theorem A.3.2 by conditioning on the random variable $S$ in the respective expectations that need to be considered, these are $\mathbb{E} V_{t}$ (for $\left.\mathbb{E} V_{t}=1\right), \mathbb{E}\left(\exp \left(u_{1} \xi_{t_{1}}+\cdots+u_{n} \xi_{t_{n}}\right)\right)$ with $\xi_{t}=S W_{t}-S^{2} \sigma^{2}(t) / 2$ (for stationarity, cf. Proposition A.3.1) and $\mathbb{E} \bigvee_{i=1}^{n}\left(V_{t_{i}} / x_{i}\right)$ (for the law of $X$, cf. 1.6).

Remark A.3.4. It is also possible to admit a point mass $G(0+)$ at 0 in the law of $S$ in Corollary A.3.3. However, we avoid adding a trivial component.

\section{A.4. Extremal points and the Krein-Milman-Theorem}

The vector space $\mathbb{R}^{T \times T}$ (endowed with the topology of pointwise convergence) is locally convex and Hausdorff for arbitrary sets $T$. Therefore, the Krein-Milman theorem applies to $E=\mathbb{R}^{T \times T}$ as considered in Section 3.5 .

Definition A.4.1. Let $E$ be a vector space and $K \subset E$ convex. An element $k \in K$ is called extremal point of $K$ if the following implication holds

$$
k_{1}, k_{2} \in K, \alpha \in(0,1), k=\alpha k_{1}+(1-\alpha) k_{2} \quad \Longrightarrow \quad k_{1}=k_{2}=k
$$

The set of extremal points of $K$ will be denoted by $\operatorname{ex}(K)$.

Theorem A.4.2 (Krein-Milman, cf. [5] 2.5.5.). Let $E$ be a locally convex Hausdorff topological vector space over $\mathbb{R}$. Then every compact, convex, non-empty subset $K$ in $E$ is the closed convex hull of its extremal points:

$$
K=\overline{\operatorname{conv}}(\operatorname{ex}(K))
$$

\section{A.5. Auxiliary results}

Here, we collect some auxiliary results in order to avoid a diversion from the leading ideas in the respective Sections 1.1, 2.3, 2.7 and 3.2. The following proposition shows how to derive from [66] a characterization of the class of stable tail dependence functions 1.2 . 
Proposition A.5.1. Let $M$ be a non-empty finite set. The function $\ell:[0, \infty)^{M} \rightarrow$ $\mathbb{R}$ is a stable tail dependence function if and only if the following conditions are satisfied:

(i) $\ell$ is sublinear, i.e. $\ell(x+y) \leq \ell(x)+\ell(y)$ for $x, y \in[0, \infty)^{M}$,

(ii) $\ell$ is homogeneous of degree 1 ,

(iii) $\ell$ is max-completely alternating,

i.e. $\ell$ is completely alternating on the semigroup $\left([0, \infty), \vee, \mathbf{1}_{\emptyset}\right)$,

(iv) $\ell\left(\mathbf{1}_{\{t\}}\right)=1$ for all $t \in M$.

Proof. Let $\ell$ be a stable tail dependence function. By definition 1.2 it is clear that $\ell$ is sublinear and homogeneous (since $\ell$ is a mixture of sublinear and homogeneous functions) and that $\ell\left(\mathbf{1}_{\{t\}}\right)=1$ for all $t \in M$ (due to standard Fréchet marginals). Because of [66, Theorem 1] the function $\ell$ can be expressed as support function $\ell(x)=\sup \{\langle x, y\rangle: y \in \mathcal{K}\}$ of a convex compact set $\mathcal{K}$, which is a dependency set and hence a max-zonoid, cf. [66, Definition 1]. Finally, [66, Theorem 7] implies that $\ell$ is max-completely alternating.

Reversely, let $\ell$ satisfy the properties (i) to (iv). Because $\ell$ is sublinear and homogeneous, $\ell$ can be expressed as support function $\ell(x)=\sup \{\langle x, y\rangle: y \in \mathcal{K}\}$ of a convex compact set $\mathcal{K} \subset[0, \infty)^{M}$ (cf. [87, Theorem 1.7.1]). Then [66, Theorem 7] implies that $\mathcal{K}$ is a max-zonoid since $\ell$ was max-completely alternating. Finally, the normalization $\ell\left(\mathbf{1}_{\{t\}}\right)=1$ implies that $\mathcal{K}$ is even a dependency set. Hence $\ell$ is a stable tail dependence function, cf. [66, Theorem 1].

Lemma A.5.2. Let $M$ be a non-empty finite set and $\ell:[0, \infty)^{M} \rightarrow \mathbb{R}$. Then $\ell$ is max-completely alternating if and only if for all $\emptyset \neq L=\left\{t_{1}, t_{2}, \ldots, t_{l}\right\} \subset M$ and $v, y \in[0, \infty)^{M}$ with $v \geq y$ (componentwise)

$$
\Delta_{v_{\left\{t_{1}\right\}}}^{\vee} \Delta_{v_{\left\{t_{2}\right\}}}^{\vee} \ldots \Delta_{v_{\left\{t_{l}\right\}}}^{\vee} \ell(y)=\sum_{I \subset L}(-1)^{|I|} \ell\left(y \vee v_{I}\right) \leq 0
$$

where $v_{A}:=v \cdot \mathbf{1}_{A}$ (componentwise).

Proof. The semigroup $\left([0, \infty)^{M}, \vee, \mathbf{1}_{\emptyset}\right)$ is generated by the functions $\left\{w_{\{t\}}\right\}$ for $t \in$ $M$ and $w \in[0, \infty)^{M}$ that have only one non-zero component. Therefore, maxcomplete-alternation of $\ell$ is equivalent to $\Delta_{w_{\left\{t_{1}\right\}}^{(1)}}^{\vee} \Delta_{w_{\left\{t_{2}\right\}}^{(2)}}^{\vee} \ldots \Delta_{w_{\left\{t_{n}\right\}}^{(n)}}^{\vee} \ell(y) \leq 0$ for $w^{(i)} \in$ $[0, \infty)^{M}$ and $t_{i} \in M(i=1, \ldots, n)$ and $n \geq 1$ (cf. Lemma A.1.7). Since $\Delta_{a_{\{t\}}}^{\vee} \Delta_{b_{\{t\}}}^{\vee}=$ $\Delta_{a_{\{t\}} \wedge b_{\{t\}}}^{\vee}$, it suffices to choose $n \leq|M|$ and to involve for each $t \in M$ at most one generator $w_{\{t\}}$. Hence, max-complete alternation of $\ell$ is equivalent to A.5 for all 
$\emptyset \neq L=\left\{t_{1}, t_{2}, \ldots, t_{l}\right\} \subset M$ and $v, y \in[0, \infty)^{M}$. The values of $v$ on $M \backslash L$ do not enter A.5 and may be chosen arbitrary, whereas the expression is automatically zero if $v_{t} \leq y_{t}$ for some $t \in L$. This finishes the proof.

Lemma A.5.3. Let $M$ be a finite set and $\psi: \mathcal{F}(M) \rightarrow \mathbb{R}$ be a function on the subsets of $M$. Let $K, L \subset M$ with $K \cap L=\emptyset$. Then

$$
\sum_{I \subset L}(-1)^{|I|+1} \psi(K \cup I)=\sum_{J \subset M \backslash(K \cup L)}\left(\sum_{I \subset L \cup J}(-1)^{|I|+1} \psi((M \backslash(L \cup J)) \cup I)\right) .
$$

Proof. (by induction on the number of elements in $M$ ) The assertion is true for $M=$ $\emptyset$. For further considerations, we abbreviate $\sigma(\psi, L, K):=\sum_{I \subset L}(-1)^{|I|+1} \psi(K \cup I)$, such that A.6 reads as

$$
\sigma(\psi, L, K)=\sum_{J \subset M \backslash(K \cup L)} \sigma(\psi, L \cup J, M \backslash(L \cup J)) .
$$

Consider now a finite set $M$ and $N=M \cup\{a\}$, where $a$ is not yet contained in $M$. Let $K, L \subset N$ with $K \cap L=\emptyset$. We need to establish

$$
\sigma(\psi, L, K)=\sum_{J \subset N \backslash(K \cup L)} \sigma(\psi, L \cup J, N \backslash(L \cup J))
$$

1st case: $a \in K$. Set $\psi_{a}(C):=\psi(C \cup\{a\})$ and $K_{a}:=K \backslash\{a\}$. Then A.8 follows from the induction hypothesis A.7 with $K$ replaced by $K_{a}$ and $\psi$ replaced by $\psi_{a}$.

2nd case: $a \in L$. Set $\psi_{a}(C):=\psi(C \cup\{a\})$ and $L_{a}:=L \backslash\{a\}$. Then the 1.h.s. of A.8 is given by $\sigma\left(\psi, K, L_{a}\right)-\sigma\left(\psi_{a}, K, L_{a}\right)$ and the r.h.s. by $\sum_{J \subset M \backslash\left(K \cup L_{a}\right)} \sigma\left(\psi, L_{a} \cup\right.$ $\left.J, M \backslash\left(L_{a} \cup J\right)\right)-\sigma\left(\psi_{a}, L_{a} \cup J, M \backslash\left(L_{a} \cup J\right)\right)$. Both terms coincide due to the induction hypothesis A.7) applied to $\psi$ and $L_{a}$ and to $\psi_{a}$ and $L_{a}$ (instead of $\psi$ and $L$ therein).

3rd case: $a \in N \backslash(K \cup L)$. Then the r.h.s. of A.8 splits according to whether $a \notin$ $J$ or $a \in J$ into

$$
\begin{aligned}
& \sum_{J \subset N \backslash(K \cup L)} \sigma(\psi, L \cup J, N \backslash(L \cup J))=\left[\sum_{J \subset M \backslash(K \cup L)} \sigma\left(\psi_{a}, L \cup J, M \backslash(L \cup J)\right)\right] \\
& +\left[\sum_{J \subset M \backslash(K \cup L)} \sigma(\psi, L \cup J, M \backslash(L \cup J))-\sigma\left(\psi_{a}, L \cup J, M \backslash(L \cup J)\right)\right]
\end{aligned}
$$

and therefore equals the r.h.s. of the induction hypothesis A.7. 
Lemma A.5.4. Let $c \in \mathbb{R}$ and $p:[c, \infty) \rightarrow(0,1)$ such that $p$ is non-decreasing and $\lim _{x \rightarrow \infty} p(x)=1$. Let $\alpha \geq 1$. Then

$$
\lim _{x \rightarrow \infty} \frac{1-p(x)^{\alpha}}{1-p(x)}=\alpha
$$

Proof. This is an application of l'Hôpitals rule, since $\lim _{x \rightarrow \infty} \frac{1-p(x)^{\alpha}}{1-p(x)}=\lim _{t \uparrow 1} \frac{1-t^{\alpha}}{1-t}$.

Lemma A.5.5. Let $c \in \mathbb{R}$ and $p:[c, \infty) \rightarrow(0,1)$ such that $p$ is non-decreasing and $\lim _{x \rightarrow \infty} p(x)=1$. Let $f:[c, \infty) \rightarrow[1, \infty)$ be some function which is bounded from below by 1 . Then

a) If $\lim _{x \rightarrow \infty} f(x)=: \alpha$ exists, then $\lim _{x \rightarrow \infty} \frac{1-p(x)^{f(x)}}{1-p(x)}$ exists and equals $\alpha$.

b) If $\lim _{x \rightarrow \infty} \frac{1-p(x)^{f(x)}}{1-p(x)}=: \alpha$ exists, then $\lim _{x \rightarrow \infty} f(x)$ exists and equals $\alpha$.

Proof. a) Let $\varepsilon>0$. Because of $\lim _{x \rightarrow \infty} f(x)=\alpha \geq 1$, we may choose $x^{*} \in[c, \infty)$ so big that for all $x \geq x^{*}$ we have $f(x) \in[\max (\alpha-\varepsilon, 1), \alpha+\varepsilon]$. We obtain the following inequality for $x \geq x^{*}$

$$
\frac{1-p(x)^{\max (\alpha-\varepsilon, 1)}}{1-p(x)} \leq \frac{1-p(x)^{f(x)}}{1-p(x)} \leq \frac{1-p(x)^{\alpha+\varepsilon}}{1-p(x)}
$$

By Lemma A.5.4 the left-hand side converges to $\max (\alpha-\varepsilon, 1)$ whereas the righthand side converges to $\alpha+\varepsilon$ as $x \rightarrow \infty$. This shows the claim.

b) Set $g(x)=\frac{1-p(x)^{f(x)}}{1-p(x)}$. Since $f(x) \geq 1$ and $p(x) \in(0,1)$, also $g(x) \geq 1$ for all $x \in[c, \infty)$. Let $\varepsilon>0$. Because $\lim _{x \rightarrow \infty} g(x)=\alpha \geq 1$, we may choose $x^{*} \in[c, \infty)$ so big that for all $x \geq x^{*}$ we have $g(x) \in[\max (\alpha-\varepsilon, 1), \alpha+\varepsilon]$. We obtain the following inequality for $x \geq x^{*}$

$$
\frac{\log (1-\max (\alpha-\varepsilon, 1)(1-p(x)))}{\log (p(x))} \leq f(x) \leq \frac{\log (1-(\alpha+\varepsilon)(1-p(x)))}{\log (p(x))}
$$

If we consider the situation as $x$ tends to $\infty$, l'Hôpitals rule applies again, since $\lim _{x \rightarrow \infty} \frac{\log (1-\beta(1-p(x)))}{\log (p(x))}=\lim _{t \uparrow 1} \frac{\log (1-\beta(1-t))}{\log (t)}=\beta$ for $\beta=\max (\alpha-\varepsilon, 1)$ for the left-hand side and $\beta=\alpha+\varepsilon$ for the right-hand side. This shows the claim. 


\section{A.6. Further members of the Gneiting class $H_{\infty}$}

Table A.1 provides further members of the Gneiting class $H_{\infty}$ in addition to Table 4.3 In these examples both the probability density function and the corresponding scale mixture of erfc can be expressed in terms of elementary functions. As for Table 4.3. this table can be derived from known Laplace transforms in [77] when Lemma 4.5 .5 is applied to [p. 9635.2 (2)], [p. 9635.2 (9)], [p. 9675.7 (5)], [p. $9635.3(2)$ ], [p. $9635.3(3)$ with $\nu=2.5$ ], [p. 9635.3 (3) with $\nu=3$ ], [p. $9635.3(4)$ ], [p. 9665.6 (7)], [p. $9665.6(8)$ ] therein. 
Probability density $g(s) \quad$ Scale mixture $\varphi(t)=\int_{0}^{\infty} \operatorname{erfc}(s t) g(s) \mathrm{d} s$

\begin{tabular}{|c|c|c|}
\hline$\frac{1}{b-a} \mathbf{1}_{[a, b]}(s)$ & $\frac{1}{b-a}\left[(\sqrt{\pi} t)^{-1}\left(e^{-a^{2} t^{2}}-e^{-b^{2} t^{2}}\right)\right.$ & $0 \leq a<b$ \\
\hline & $+b \operatorname{erfc}(b t)-a \operatorname{erfc}(a t)]$ & \\
\hline$a^{2}\left(s^{2}+a^{2}\right)^{-3 / 2}$ & $e^{a^{2} t^{2}} \operatorname{erfc}(a t)$ & $0<a$ \\
\hline$a \sqrt{\pi} \operatorname{erfc}(a s)$ & $1-\left(\sqrt{\frac{1}{t^{2}}+\frac{1}{a^{2}}}-\frac{1}{t}\right)$ & $0<a$ \\
\hline$\frac{4 a^{3}}{\sqrt{\pi}} s^{2} e^{-a^{2} s^{2}}$ & $1-\left[\frac{2}{\pi} \arctan \left(\frac{t}{a}\right)+\frac{4}{\pi} \cdot \frac{t}{a^{2}\left(a^{2}+t^{2}\right)}\right]$ & $0<a$ \\
\hline $2 a^{4} s^{3} e^{-a^{2} s^{2}}$ & $1-\frac{t\left(2 t^{2}+3 a^{2}\right)}{2\left(t^{2}+a^{2}\right)^{3 / 2}}$ & $0<a$ \\
\hline$\frac{8 a^{5}}{3 \sqrt{\pi}} s^{4} e^{-a^{2} s^{2}}$ & $1-\left[\frac{2}{\pi} \arctan \left(\frac{t}{a}\right)+\frac{2}{3 \pi} \cdot \frac{5 a^{3} t+3 a t^{3}}{\left(a^{2}+t^{2}\right)^{2}}\right]$ & $0<a$ \\
\hline$\frac{s^{-2}\left(e^{-a^{2} s^{2}}-e^{-b^{2} s^{2}}\right)}{\sqrt{\pi}(b-a)}$ & $1-\frac{1}{\pi(b-a)}\left[t \log \left(\frac{t^{2}+b^{2}}{t^{2}+a^{2}}\right)\right.$ & $0 \leq a<b$ \\
\hline$\frac{2}{a \sqrt{\pi}} s^{-2} \sin ^{2}\left(a^{2} s^{2}\right)$ & $1-\frac{1}{\pi}\left[\arctan \left(\frac{t}{a}+1\right)+\arctan \left(\frac{t}{a}-1\right)\right.$ & $0<a$ \\
\hline & $+\frac{t}{2 a} \log \left(\frac{4 a^{4}}{t^{4}}+1\right)+\operatorname{arctanh}\left(\frac{2 a t}{2 a^{2}+t^{2}}\right.$ & \\
\hline$\frac{3}{2 a^{3} \sqrt{\pi}} s^{-4} \sin ^{2}\left(a^{2} s^{2}\right)$ & $1-\frac{1}{\pi}\left[\arctan \left(\frac{t}{a}+1\right)+\arctan \left(\frac{t}{a}-1\right)\right.$ & $0<a$ \\
\hline & $+\frac{3 t}{2 a} \arctan \left(\frac{2 a^{2}}{t^{2}}\right)-\frac{t^{3}}{8 a^{3}} \log \left(\frac{4 a^{4}}{t^{4}}+1\right)$ & \\
\hline & $\left.+\frac{1}{2} \log \left(\frac{2 a^{2}-2 a t+t^{2}}{2 a^{2}+2 a t+t^{2}}\right)\right]$ & \\
\hline
\end{tabular}

Table A.1.: Members of the Gneiting class $H_{\infty}$ (cf. 4.9 ) and their corresponding probability density function $g(s)$ on $[0, \infty)$ as scale mixtures of the complementary error function, cf. also Table 4.3 . 



\section{References}

[1] The On-Line Encyclopedia of Integer Sequences. A000110. URL http://oeis.org/ A000110

[2] O. Aichholzer and F. Aurenhammer. Classifying hyperplanes in hypercubes. SIAM J. Discrete Math., 9(2):225-232, 1996.

[3] C. D. Aliprantis and K. C. Border. Infinite Dimensional Analysis. A hitchhiker's guide. Springer, Berlin, third edition, 2006.

[4] J. Beirlant, Y. Goegebeur, J. Teugels, and J. Segers. Statistics of Extremes. Wiley Series in Probability and Statistics. John Wiley \& Sons Ltd., Chichester, 2004.

[5] C. Berg, J. P. R. Christensen, and P. Ressel. Harmonic Analysis on Semigroups, volume 100 of Graduate Texts in Mathematics. Springer-Verlag, NY, 1984.

[6] C. Berg, J. Mateu, and E. Porcu. The Dagum family of isotropic correlation functions. Bernoulli, 14(4):1134-1149, 2008.

[7] J. Blanchet and A. C. Davison. Spatial modeling of extreme snow depth. Ann. Appl. Stat., 5(3):1699-1725, 2011.

[8] T. A. Buishand, L. de Haan, and C. Zhou. On spatial extremes: with application to a rainfall problem. Ann. Appl. Stat., 2(2):624-642, 2008.

[9] J.-P. Chilès and P. Delfiner. Geostatistics. Wiley Series in Probability and Statistics: Applied Probability and Statistics. John Wiley \& Sons Inc., New York, 1999.

[10] S. Coles, J. Heffernan, and J. Tawn. Dependence measures for extreme value analyses. Extremes, 2(4):339-365, 1999.

[11] D. Cooley, P. Naveau, and P. Poncet. Variograms for spatial max-stable random fields. In Dependence in Probability and Statistics, volume 187 of Lecture Notes in Statist., pages 373-390. Springer, New York, 2006.

[12] H. Cramér and M. R. Leadbetter. Stationary and Related Stochastic Processes. Sample Function Properties and Their Applications. John Wiley \& Sons Inc., New York, 1967.

[13] D. J. Daley and D. Vere-Jones. An Introduction to the Theory of Point Processes. Vol. II. Probability and its Applications (New York). Springer, New York, second edition, 2008.

[14] R. A. Davis and T. Mikosch. The extremogram: a correlogram for extreme events. 
Bernoulli, 15(4):977-1009, 2009.

[15] L. de Haan. A spectral representation for max-stable processes. Ann. Probab., 12(4): 1194-1204, 1984.

[16] L. de Haan and A. Ferreira. Extreme Value Theory. An Introduction. Springer Series in Operations Research and Financial Engineering. Springer, New York, 2006.

[17] L. de Haan and S. I. Resnick. Limit theory for multivariate sample extremes. $Z$. Wahrscheinlichkeitstheorie und Verw. Gebiete, 40(4):317-337, 1977.

[18] C. Dombry, F. Éyi-Minko, and M. Ribatet. Conditional simulation of max-stable processes. Biometrika, 100:111-124, 2013.

[19] H. Drees and X. Huang. Best attainable rates of convergence for estimators of the stable tail dependence function. J. Multivariate Anal., 64(1):25-47, 1998.

[20] A. Ehlert and M. Schlather. A constructive proof for the extremal coefficient of a dissipative max-stable process on $\mathrm{Z}$ being a set covariance. Courant Research Centre PEG - Discussion Papers 31, 2010. URL http://www2.vwl.wiso.uni-goettingen. de/courant-papers/CRC-PEG_DP_31.pdf

[21] J. H. J. Einmahl and J. Segers. Maximum empirical likelihood estimation of the spectral measure of an extreme-value distribution. Ann. Statist., 37(5B):2953-2989, 2009.

[22] J.H.J. Einmahl, A. Krajina, and J. Segers. An M-estimator for tail dependence in arbitrary dimensions. The Annals of Statistics, 40(3):1764-1793, 2012.

[23] P. Embrechts, C. Klüppelberg, and T. Mikosch. Modelling Extremal Events, volume 33 of Applications of Mathematics. Springer-Verlag, Berlin, 1997.

[24] X. Emery. On the existence of mosaic and indicator random fields with spherical, circular, and triangular variograms. Math. Geosci., 42(8):969-984, 2010.

[25] S. Engelke, Z. Kabluchko, and M. Schlather. Maxima of independent, non-identically distributed Gaussian vectors. arXiv1205.0947v1, 2012. URL http://arxiv . org/abs/ 1205.0947 .

[26] S. Engelke, A. Malinowski, Z. Kabluchko, and M. Schlather. Estimation of HüslerReiss distributions and Brown-Resnick processes. arXiv1207.6886v1, 2012. URL http: //arxiv.org/abs/1207.6886

[27] S. Engelke, A. Malinowski, M. Oesting, and M. Schlather. Representations of maxstable processes based on single extreme events. arXiv1209.2303v1, 2012. URL http: //arxiv.org/abs/1209.2303

[28] M. Falk. On the generation of a multivariate extreme value distribution with prescribed tail dependence parameter matrix. Statist. Probab. Lett., 75(4):307-314, 2005.

[29] V. Fasen, C. Klüppelberg, and M. Schlather. High-level dependence in time series models. Extremes, 13(1):1-33, 2010.

[30] H. Ferreira. Generating multivariate extrema value distributions. arXiv1203.1875v1, 2012. URL http://arxiv.org/abs/1203.1875.

[31] R. A. Fisher and L. H. C. Tippett. Limiting forms of the frequency distribution of the largest or smallest member of a sample. Math. Proc. Cambridge Philos. Soc., 24: 180-190, 1928. 
[32] J. Geffroy. Contribution à la théorie des valeurs extrêmes. Publ. Inst. Statist. Univ. Paris, 7/8:37-185, 1958/1959.

[33] M. G. Genton, Y. Ma, and H. Sang. On the likelihood function of Gaussian max-stable processes. Biometrika, 98(2):481-488, 2011.

[34] Ч. I. Gīhman and A. V. Skorohod. The Theory of Stochastic Processes. I. SpringerVerlag, New York, 1974. Translated from the Russian by S. Kotz, Die Grundlehren der mathematischen Wissenschaften, Band 210.

[35] E. Giné, M. G. Hahn, and P. Vatan. Max-infinitely divisible and max-stable sample continuous processes. Probab. Theory Related Fields, 87(2):139-165, 1990.

[36] B. Gnedenko. Sur la distribution limite du terme maximum d'une série aléatoire. Ann. of Math. (2), 44:423-453, 1943.

[37] T. Gneiting. Radial positive definite functions generated by Euclid's hat. J. Multivariate Anal., 69(1):88-119, 1999.

[38] T. Gneiting. Isotropic correlation functions on $d$-dimensional balls. Adv. Appl. Probab., 31(3):625-631, 1999.

[39] T. Gneiting. On the derivatives of radial positive definite functions. J. Math. Anal. Appl., 236(1):86-93, 1999.

[40] T. Gneiting, Z. Sasvári, and M. Schlather. Analogies and correspondences between variograms and covariance functions. Adv. Appl. Probab., 33:617-630, 2001.

[41] B. I. Golubov. On Abel-Poisson type and Riesz means. Anal. Math., 7(3):161-184, 1981.

[42] I. S. Gradshteyn and I. M. Ryzhik. Table of Integrals, Series and Products. Elsevier/Academic Press, Amsterdam, seventh edition, 2007. Translated from the Russian, Translation edited and with a preface by Alan Jeffrey and Daniel Zwillinger.

[43] C. Huang, H. Zhang, and S. M. Robeson. On the validity of commonly used covariance and variogram functions on the sphere. Math. Geosci., 43(6):721-733, 2011.

[44] J. Hüsler and R.-D. Reiss. Maxima of normal random vectors: between independence and complete dependence. Statist. Probab. Lett., 7(4):283-286, 1989.

[45] Y. Jiao, F. H. Stillinger, and S. Torquato. Modeling heterogeneous materials via two-point correlation functions: basic principles. Phys. Rev. E (3), 76(3):031110, 13, 2007.

[46] C. N. Jones, E. C. Kerrigan, and J. M. Maciejowski. Equality set projection: a new algorithm for the projection of polytopes in halfspace representation. Technical Report CUED/F-INFENG/TR. 463, Department of Engineering, University of Cambridge, 2004. URL http://www-control.eng.cam.ac.uk/ cnj22/docs/resp_mar_04_15. pdf.

[47] P. E. Jupp and K. V. Mardia. Maximum likelihood estimators for the matrix von Mises-Fisher and Bingham distributions. Ann. Statist., 7(3):599-606, 1979.

[48] Z. Kabluchko. Spectral representations of sum- and max-stable processes. Extremes, 12(4):401-424, 2009.

[49] Z. Kabluchko. Extremes of independent Gaussian processes. Extremes, 14(3):285-310, 2011. 
[50] Z. Kabluchko and M. Schlather. Ergodic properties of max-infinitely divisible processes. Stochastic Process. Appl., 120(3):281-295, 2010.

[51] Z. Kabluchko and S. Stoev. Minimal spectral representations of infinitely divisible and max-infinitely divisible processes. arXiv1207.4983v1, 2012. URL http://arxiv. org/abs/1207.4983.

[52] Z. Kabluchko, M. Schlather, and L. de Haan. Stationary max-stable fields associated to negative definite functions. Ann. Probab., 37(5):2042-2065, 2009.

[53] J. Kim and D. Pollard. Cube root asymptotics. Ann. Statist., 18(1):191-219, 1990.

[54] J. F. C. Kingman. Poisson Processes, volume 3 of Oxford Studies in Probability. The Clarendon Press Oxford University Press, New York, 1993. Oxford Science Publications.

[55] C. Lantuéjoul, J.-N. Bacro, and L. Bel. Storm processes and stochastic geometry. Extremes, 14(4):413-428, 2011.

[56] M. Ledoux and M. Talagrand. Probability in Banach spaces. Isoperimetry and Processes, volume 23 of Ergebnisse der Mathematik und ihrer Grenzgebiete (3) [Results in Mathematics and Related Areas (3)]. Springer-Verlag, Berlin, 1991.

[57] K. V. Mardia and C. G. Khatri. Uniform distribution on a Stiefel manifold. J. Multivariate Anal., 7(3):468-473, 1977.

[58] K. Z. Markov. On the "triangular" inequality in the theory of two-phase random media. Annuaire Univ. Sofia Fac. Math. Inform., 89(1-2):159-166 (1998), 1995.

[59] G. Matheron. The intrinsic random functions and their applications. Adv. Appl. Probab., 5:439-468, 1973.

[60] G. Matheron. Suffit-il pour une covariance d'être de type positif? Etud. Géostat. V, Séminaire CFSG Geostat., Sciences de la Terre Inf. Nancy, 1988.

[61] G. Matheron. Une conjecture sur la covariance d'un ensemble aléatoire. Cahiers de Géostatique, Fasc. 3, Ecole des Mines de Paris, pages 107-113, 1993.

[62] A. J. McNeil and J. Nešlehová. Multivariate Archimedean copulas, $d$-monotone functions and $l_{1}$-norm symmetric distributions. Ann. Statist., 37(5B):3059-3097, 2009.

[63] K. S. Miller and S. G. Samko. Completely monotonic functions. Integral Transform. Spec. Funct., 12(4):389-402, 2001.

[64] Y. Mittal. A class of isotropic covariance functions. Pacific J. Math., 64(2):517-538, 1976.

[65] I. Molchanov. Theory of Random Sets. Probability and its Applications (New York). Springer-Verlag, London Ltd., 2005.

[66] I. Molchanov. Convex geometry of max-stable distributions. Extremes, 11(3):235-259, 2008.

[67] I. Molchanov and K. Stucki. Stationarity of multivariate particle systems. arXiv1201.4765v1, 2012. URL http://arxiv.org/abs/1201.4765.

[68] L. Nachbin. The Haar Integral. Robert E. Krieger Publishing Co., Huntington, N.Y., 1976. Translated from the Portuguese by Lulu Bechtolsheim, Reprint of the 1965 edition. 
[69] P. Naveau, A. Guillou, D. Cooley, and J. Diebolt. Modelling pairwise dependence of maxima in space. Biometrika, 96(1):1-17, 2009.

[70] M. Oesting and M. Schlather. Conditional sampling for max-stable processes with a mixed moving maxima representation. arXiv1202.5023v2, 2012. URL http://arxiv. org/abs/1202.5023.

[71] M. Oesting, Z. Kabluchko, and M. Schlather. Simulation of Brown-Resnick processes. Extremes, 15(1):89-107, 2012.

[72] Encyclopedia of Mathematics. Haar measure. D.P. Zhelobenko and A.I. Shtern (originator). http://www.encyclopediaofmath.org/index.php?title= Haar_measure\&oldid=19105

[73] Encyclopedia of Mathematics. Isotropy group. Ü. Lumiste (originator). http://www. encyclopediaofmath.org/index.php?title=Isotropy_group\&oldid=17780

[74] I. Papastathopoulos and J. A. Tawn. Dependence properties of multivariate max-stable distributions. arXiv1210.8268, 2012. URL http://arxiv.org/abs/1210.8268.

[75] J. Pickands. Multivariate extreme value distributions. In Proceedings 43rd Session International Statistical Institute, volume 2, pages 859-878, 1981.

[76] G. Pólya. Remarks on characteristic functions. In Proceedings of the Berkeley Symposium on Mathematical Statistics and Probability, 1945, 1946, pages 115-123, Berkeley and Los Angeles, 1949. University of California Press.

[77] A. D. Polyanin and A. V. Manzhirov. Handbook of Integral Equations. Chapman \& Hall/CRC, Boca Raton, FL, second edition, 2008.

[78] E. Porcu and R. L. Schilling. From Schoenberg to Pick-Nevanlinna: toward a complete picture of the variogram. Bernoulli, 17(1):441-455, 2011.

[79] S. I. Resnick. Extreme Values, Regular Variation and Point Processes. Series in Operations Research and Financial Engineering. Springer, NY, 1987.

[80] R. Schaback. Creating surfaces from scattered data using radial basis functions. In Mathematical Methods For Curves And Surfaces (Ulvik, 1994), pages 477-496. Vanderbilt Univ. Press, Nashville, TN, 1995.

[81] M. Scheuerer. A Comparison of Models and Methods for Spatial Interpolation in Statistics and Numerical Analysis. PhD thesis, Georg-August-Universität Göttingen, 2010. URL http://webdoc.sub.gwdg.de/diss/2010/scheuerer/scheuerer.pdf

[82] R. L. Schilling, R. Song, and Z. Vondraček. Bernstein Functions. Theory and applications, volume 37 of de Gruyter Studies in Mathematics. Walter de Gruyter \& Co., Berlin, 2010.

[83] M. Schlather. Models for stationary max-stable random fields. Extremes, 5(1):33-44, 2002.

[84] M. Schlather. Construction of covariance functions and unconditional simulation of random fields. In Advances and Challenges in Space-time Modelling of Natural Events, volume 207 of Lecture Notes in Statist., pages 25-54. Springer, Berlin and Heidelberg, 2012.

[85] M. Schlather and J. Tawn. Inequalities for the extremal coefficients of multivariate extreme value distributions. Extremes, 5(1):87-102, 2002. 
[86] M. Schlather and J.A. Tawn. A dependence measure for multivariate and spatial extreme values: Properties and inference. Biometrika, 90:139-156, 2003.

[87] R. Schneider. Convex Bodies: The Brunn-Minkowski Theory. Cambridge University Press, Cambridge, 1993.

[88] R. Schneider and W. Weil. Stochastic And Integral Geometry. Probability and its Applications. Springer-Verlag, Berlin, 2008.

[89] M. Sibuya. Bivariate extreme statistics. I. Ann. Inst. Statist. Math. Tokyo, 11:195-210, 1960.

[90] R.L. Smith. Max-stable processes and spatial extremes. Unpublished Manuscript, 1990.

[91] S. A. Stoev. On the ergodicity and mixing of max-stable processes. Stochastic Process. Appl., 118(9):1679-1705, 2008.

[92] S. A. Stoev and M. S. Taqqu. Extremal stochastic integrals: a parallel between maxstable processes and $\alpha$-stable processes. Extremes, 8(4):237-266 (2006), 2005.

[93] K. Strokorb and M. Schlather. Characterizing extremal coefficient functions and extremal correlation functions. arXiv1205.1315v1, 2012. URL http://arxiv.org/abs/ 1205.1315

[94] K. Strokorb and M. Schlather. An exceptional max-stable process fully parameterized by its extremal coefficients. Submitted, 2013.

[95] J. Tiago de Oliveira. Structure theory of bivariate extremes; extensions. Est. Mat. Estat. Econ., 7:165-95, 1962/63.

[96] Y. Wang and S. A. Stoev. On the structure and representations of max-stable processes. Adv. in Appl. Probab., 42(3):855-877, 2010.

[97] Y. Wang, P. Roy, and S. A. Stoev. Ergodic properties of sum- and max-stable stationary random fields via null and positive group actions. Ann. Probab., 41(1):206-228, 2013.

[98] H. Wendland. Scattered Data Approximation, volume 17 of Cambridge Monographs on Applied and Computational Mathematics. Cambridge University Press, Cambridge, 2005.

[99] D.V. Widder. The Laplace Transform. Princeton mathematical series. Princeton University Press, Princeton, N. J., 1946.

[100] R. E. Williamson. Multiply monotone functions and their Laplace transforms. Duke Math. J., 23:189-207, 1956.

[101] A. M. Yaglom. Second-order homogeneous random fields. In Proc. 4th Berkeley Sympos. Math. Statist. and Prob., Vol. II, pages 593-622. University of California Press, Berkeley, California, 1961.

[102] V. P. Zastavnyi and E. Porcu. Characterization theorems for the Gneiting class of space-time covariances. Bernoulli, 17(1):456-465, 2011.

[103] W. zu Castell. Recurrence relations for radial positive definite functions. J. Math. Anal. Appl., 271(1):108-123, 2002. 


\title{
List of abbreviations and symbols
}

\author{
Numbers \\ $\mathbb{N} \quad$ natural numbers $\mathbb{N}=\{1,2,3, \ldots\}$ \\ $\mathbb{R} \quad$ real numbers \\ $\mathbb{R}_{+} \quad$ non-negative real numbers $\mathbb{R}_{+}=[0, \infty)$
}

Sets

$\mathcal{F}(T)$

$|M|$

$\mathcal{P}(T)$

$M$

T

set of finite subsets of $T$

cardinality of a finite set $M$

power set of $T$ (endowed with the product topology from $\{0,1\}^{T}$ )

non-empty finite subset of $T$

arbitrary (index) set

\section{Norms}

$\|\cdot\|$

reference norm on $\mathbb{R}^{M}$

8

$|\cdot|$

absolute value of a real number

$\|\cdot\|_{2}$

Euclidean norm

$\|\cdot\|_{\infty}$

maximum norm

$\|\cdot\|_{L^{1}}$

$L^{1}$ norm on a measure space

\section{Operations and operators}

$(\cdot)^{\mathrm{T}}$
$(\cdot)_{+}$
$\Delta_{K}$
$\Delta_{s}$
$\langle x, y\rangle$
$\mathcal{L}(F)$
$\lfloor\cdot\rfloor$
$\quad \bigvee$

transpose of a matrix

$\max (0, \cdot)$

$\Delta_{K} \quad$ difference operator w.r.t. union of sets $\Delta_{K} f=f(\cdot)-f(\cdot \cup K)$

difference operator w.r.t. semigroup operation $\Delta_{s} f=f(\cdot)-f(\cdot \circ s) 121$

$\langle x, y\rangle$

standard scalar product of $x$ and $y$

Laplace transform $\mathcal{L}(F)(x)=\int_{0}^{\infty} \exp (-x t) \mathrm{d} F(t)$

of a non-decreasing (distribution) function $F$

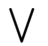

largest previous integer of a real number (floor function)

maximum 


$\begin{array}{ll}\wedge & \text { minimum } \\ \mathrm{TB}_{k}^{d} & \text { turning bands operator from dimension } k \text { to } d \text {, see Definition 4.4.1 } \\ \vee & \text { maximum (componentwise where applicable) } \\ \wedge & \text { minimum (componentwise where applicable) }\end{array}$

\section{Constants and special functions}

$\arcsin (x)$

inverse sine function $\sin ^{-1}(x)$

$\arctan (x)$

inverse tangent function $\tan ^{-1}(x)$

$\operatorname{arctanh}(x)$

inverse hyperbolic tangent function $\tanh ^{-1}(x)$

$\beta_{d}$

$\beta_{d}=\Gamma(d / 2) / \Gamma((d+1) / 2) / \sqrt{\pi}$

$\operatorname{erf}(x)$

$\operatorname{erfc}(x)$

error function $\operatorname{erf}(x)=\frac{2}{\sqrt{\pi}} \int_{0}^{x} e^{-t^{2}} \mathrm{~d} t$

$\Gamma(x)$

$\kappa_{d}$

complementary error function $\operatorname{erfc}(x)=1-\operatorname{erf}(x)=\frac{2}{\sqrt{\pi}} \int_{x}^{\infty} e^{-t^{2}} \mathrm{~d} t$

$b_{d}$

gamma function $\Gamma(x)=\int_{0}^{\infty} t^{x-1} e^{-t} \mathrm{~d} t$

volume of the $d$-dimensional unit ball $\kappa_{d}=\pi^{d / 2} / \Gamma(1+d / 2)$

volume of the $d$-dimensional ball of unit diameter,

$b_{d}=\kappa_{d} / 2^{d}=(\sqrt{\pi} / 2)^{d} / \Gamma(1+d / 2)$

$B_{n}$ $n$th Bell number

$K_{\nu}(x) \quad$ modified Bessel function of the second kind (cf. [98, p. 52]), $K_{\nu}(x)=\int_{0}^{\infty} e^{-x \cosh (t)} \cosh (\nu t) \mathrm{d} t$ with $\cosh (t)=\left(e^{t}+e^{-t}\right) / 2$

\section{Probability}

$\begin{array}{ll}\text { Cov } & \text { covariance } \\ \mathbb{E} & \text { expectation } \\ \mathbb{P} & \text { probability law } \\ \text { Var } & \text { variance } \\ \text { i.i.d. } & \text { independent and identically distributed }\end{array}$

\section{Stochastic processes and characteristics}

$\chi$

$\chi^{(X)}$

$\chi_{\Pi}$

$\eta$

$\gamma$

$\rho$

$\sigma^{2}(t)$

$\theta$

$\theta^{(u)}$

W

$X$

$X^{*}$ tail correlation function

tail correlation function of the process $X$

51

51

TCF associated to a partition $\Pi$, see Lemma 3.5 .2

variogram of a Gaussian process $W$ with stationary increments

stochastic process, often simple max-stable

spectrally discrete stochastic process

(associated to coefficients $\tau_{L}^{M}$ or to an ECF $\theta$ ) 
$X^{(u)} \quad$ a spectrally discrete stochastic process

(associated to coefficients $\tau_{L}^{M}(u)$ or to an ECF $\theta^{(u)}$ )

\section{Notation related to max-stable distributions}

$\mathcal{K} \quad$ dependency set, see equation (2.18)

$\mathcal{K}^{*} \quad$ dependency set of the process $X^{*}$

$\mathcal{K}^{(u)}$

dependency set of the process $X^{(u)}$

$\mathcal{K}_{M}$

(finite-dimensional) dependency set

$\ell_{M}^{*}$

stable tail dependence function of $\left\{X_{t}^{*}\right\}_{t \in M}$

46

$\ell_{M}^{(u)}$

stable tail dependence function of $\left\{X_{t}^{(u)}\right\}_{t \in M}$

$\ell_{M}$

stable tail dependence function

$\tau_{L}^{M}$

$\tau_{L}^{M}(u)$

matrix coefficients of the f.d.d. of a consistent max-linear model

coefficients describing the f.d.d. of a consistent max-linear model

$H_{M}^{*}$ finite-dimensional spectral measure of the process $X^{*}$

$H_{M}^{(u)}$

finite-dimensional spectral measure of the process $X^{(u)}$

$H_{M}$

(finite-dimensional) spectral measure

\section{Sets of functions on $T \times T$ or $\mathcal{F}(T)$}

$\operatorname{BIN}(T)$

set of uncentered and normalized covariance functions on $T \times T$ of binary processes

$\operatorname{MAX}(T)$

set of tail correlation functions on $T \times T$ stemming from simple max-stable processes

$\mathrm{CF}_{+}(T)$

set of non-negative correlation functions on $T \times T$

set of tail correlation functions on $T \times T$ stemming from processes with no jump at the upper endpoint

$\Theta(T) \quad$ set of all ECFs on $\mathcal{F}(T)$, see equation (2.2)

$\Theta^{(u)}(T)$

set of all directional ECFs on $\mathcal{F}(T)$ w.r.t. $u$, see equation (2.26)

Classes of radial functions on $[0, \infty)$

$\Phi_{\infty} \quad$ intersection of all classes $\Phi_{d}$

$\Phi_{d}$

$B_{d}$

$H_{\infty}$

$H_{d}$

$h_{d}$

$M_{d}$

$M_{d}^{o}$ set of continuous correlation functions on $\mathbb{R}^{d}$

depending only on the radius 
$V_{d} \quad$ Mittal-Berman class

\section{Miscellaneous}

$(\Omega, \mathcal{A}, \nu, V) \quad$ spectral representation of a max-stable process 10

$\left(\mathbb{R}^{d}, \mathcal{B}\left(\mathbb{R}^{d}\right), \mathrm{d} z\right) \quad$ Lebesgue measure on the Borel $\sigma$-algebra of $\mathbb{R}^{d} \quad 11$

$\mathcal{B}(\cdot) \quad$ Borel $\sigma$-algebra of a topological space

$\operatorname{conv}(\cdot)$

convex hull

$\delta_{s t}$

Kronecker delta $\delta_{s t}=\mathbb{1}_{s=t}$

$\mathbf{1}_{A}$

indicator function of a set $A$

$\mathbb{1}_{\mathscr{A}}$

indicator function of an event $\mathscr{A}$

$\operatorname{ex}(\cdot)$

$\mathcal{H}_{A}(\theta)$

extremal points of a convex set

$\mathcal{H}_{A}\left(\theta^{(u)}\right)$

halfspace in $[0, \infty)^{T}$ depending on $\theta$ and $A$

$\nu_{d}(\cdot)$

halfspace in $[0, \infty)^{T}$ depending on $\theta^{(u)}$ and $A$

$\overline{\operatorname{conv}}(\cdot)$

$d$-dimensional volume w.r.t. the Lebesgue measure on $\mathbb{R}^{d}$

closed convex hull

$\Pi(T)$

$\Pi$

set of partitions of a set $T$

partition of a set

$\beta$

intensity of a Poisson hyperplane mosaic

$\mathcal{E}_{A}(\theta)$

hyperplane in $[0, \infty)^{T}$ depending on $\theta$ and $A$

hyperplane in $[0, \infty)^{T}$ depending on $\theta^{(u)}$ and $A$

$\operatorname{pr}_{M}$

$\Psi$ natural projection from $\mathbb{R}^{T}$ to $\mathbb{R}^{M}$ for $M \subset T$

map from $[0, \infty)^{\mathcal{F}(T)}$ to $[0, \infty)^{T \times T}$ mapping ECFs to TCFs

$\sigma_{k}^{d}$

uniform distribution on the Stiefel manifold $V_{k}\left(\mathbb{R}^{d}\right)$

$\operatorname{Stab}(\cdot)$

stabilizer subgroup

$B_{r}^{d}(o)$

$d$-dimensional ball of (Euclidean) radius $r$ centered at the origin $o \in \mathbb{R}^{d}$

C

typical cell of a Poisson hyperplane mosaic

$C(\cdot)$

set of continuous functions on a topological space

$C_{\text {amax }}(\cdot)$

set of continuous functions on a topological space

that have a unique argmax

Gt

orbit of $t$ under the action of a group $G$

$S^{n}$

$n$-sphere (unit sphere w.r.t. Euclidean norm in $\mathbb{R}^{n+1}$ )

$S_{M} \quad$ reference sphere in $[0, \infty)^{M}$

$u_{A}$

$V_{k}\left(\mathbb{R}^{d}\right)$

$u_{A}=u \cdot \mathbf{1}_{A}$ (componentwise) for $u \in \mathbb{R}^{T}$ and $A \subset T$

\section{Abbreviations}

BR

Brown-Resnick (process)

EBG

extremal binary Gaussian (process)

$\mathrm{ECF}$

extremal coefficient function

EG

extremal Gaussian (process)

l.h.s.

left hand side 
r.h.s. right hand side

TCF

tail correlation function

VBR

variance-mixed Brown-Resnick (process)

w.r.t. with respect to

As a convention we denote the sets of e.g. real-valued, [0, $\infty$-valued, $\{0,1\}$-valued functions on a set $T$ by $\mathbb{R}^{T},[0, \infty]^{T}$ and $\{0,1\}^{T}$, respectively. Elements therein are addressed as $x=\left(x_{t}\right)_{t \in T}$. 



\section{Index}

$G$-invariant, 106

$\alpha$-times monotone, 124

$\chi$-measure, 1, 51

$n$-times monotone

for intervals, 124

for open intervals, 123

absolutely monotone

for intervals, 124

for open intervals, 123

Bell number, 69

Bernstein function, 28

BR process, 12

for group actions, 107

Brown-Resnick process, 12

for group actions, 107

Cantor cube, 25

capacity functional, 22

Cauchy family, 85

completely alternating, 121

in the semigroup sense, 121

on $\mathcal{F}(T), 20$

completely monotone

for intervals, 124

for open intervals, 123

in the semigroup sense, 121

control measure, 10

control measure $\nu, 9$

convex hull, 17

convex polytope, 60

correlation function, 70

dependency set, 33

finite-dimensional, 9 directional ECF, 41

directional extremal coefficient function, 41

EBG process, 11

EG process, 11]

Euclid's hat, 80

extremal binary Gaussian process, 11

extremal coefficient, 15

extremal coefficient function, 1, 15, 51

directional, 41

extremal Gaussian process, 11

extremal point, 126

extremal stochastic integral, 9

extremogram, 55

finite-dimensional distribution, 8

Fréchet marginals, 8

Fréchet type, 7

generalized extreme value distribution, 7

Gneiting class, 80

group action, 105

continuous, 105

fixpoint, 105

free, 105

transitive, 105

Gumbel type, 7

Hüsler-Reiss distribution, 33

idempotent semigroup, 122

integral representation, 25

intensity mixing distribution, 13

intrinsically stationary, 108

Laplace transform, 17, 124 
Lévy measure, 121

M3 process, 11

for group actions, 109

marginal distribution, 7

max-completely alternating, 9, 42

max-linear, 17

max-stable, 7

simple, 8

max-stable process, 7

max-zonoid, 9

mean width, 17

Mittal-Berman class, 80

Mixed Moving Maxima process, 11 for group actions, 109

Mixed Poisson storm process, 13

Moving Maxima process, 11

MPS process, 13

multiply monotone

for intervals, 124

for open intervals, 123

negative definite

in the semigroup sense, 119

on $\mathcal{F}(T), 20$

norming functions, 7

orbit, 105

partition, 67

polytope

convex, 60

positive definite

as a kernel, 70

in the semigroup sense, 119

powered error function, 85

powered exponential family, 85

principal minor, 71

process

$\mathrm{BR}, 12$

for group actions, 107

Brown-Resnick, 12

for group actions, 107

EBG, 11

$\mathrm{EG}, 11$

extremal binary Gaussian, 11

extremal Gaussian, 11

M3, 11

for group actions, 109

max-stable, 7

Mixed Moving Maxima, 11

for group actions, 109
Mixed Poisson storm, 13

Moving Maxima, 11 .

MPS, 13

spectral, 10

variance-mixed Brown-Resnick, 13

VBR, 13

Pólya's criterion, 81

Radon measure, 25

random sup-measure, 9, 10

reference norm, 8

semicharacter

bounded, 120

semigroup

abelian, 119

idempotent, 122

shape function, 11

simple, 8

simple max-stable, 8

spectral function, 10

spectral measure, 10

finite-dimensional, 8

spectral process, 10

spectral representation, 10

spectrally discrete, 17

stabilizer (subgroup), 105

stable tail dependence function, 8

standard Fréchet marginals, 8

stationary, 11

stationary increments, 12, 108

stationary isotropic, 11

Stiefel manifold, 86

support function, 9

Sylvester's criterion, 71

tail correlation function, 51

tail dependence coefficient, 51

tent function, 90

tetrahedron inequality

for TCFs, 62

three types theorem, 7

triangle inequality

for TCFs, 62

truncated power function, 85

turning bands operator, 87

uniform distribution

Stiefel manifold, 87

upper tail dependence coefficient, 51

variance mixing distribution, 13

variance-mixed Brown-Resnick process, 13 
VBR process, 13 vertex, 60

Weibull type, 7

Whittle-Matérn family, 85 
